\title{
Injury Experience in Sand and Gravel Mining, 1989 DE $\triangle 41990$
}

U.S. Department of Labor

Elizabeth H. Dole. Secretany

Mine Safety and Health Administration

IR- -1199

William J. Tattersall, Assistant Secretary

DE91 005305

Information Report

IR 1190

1980

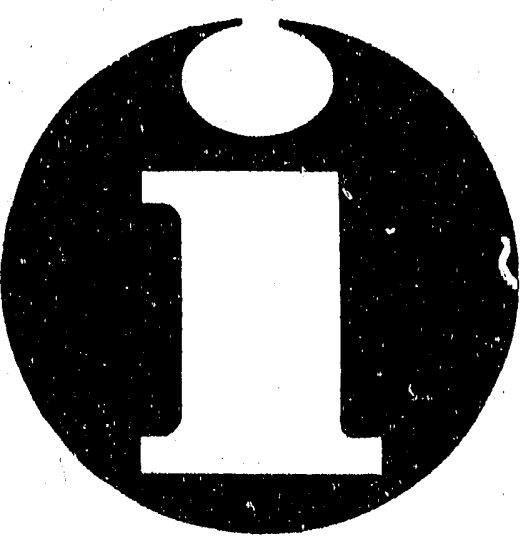

MASTER

DISTRIBUTION OF THIS DOCUMENT IS UNLIMITEE 
This publication has been reviewed and approved for distribution to the mining public by the Office of the Assistant Secretary for Mine Safety and Heal.th. 
Abstract- 1

Introduction- 1

Scope of statistics- 2

Definition of terms- 3

Tab1e reference guide-_ 7

\section{ILLUSTRATIONS}

Figure 1. Scheduled charges in 1ost workdays for 10 ss or loss of use involving hand or foot- 9

Figure 2. Quarterly mine employment and coal production report form- 10

Figure 3. Mine accident, injury, and i11ness report form-ann 11

TABLES FOR SAND AND GRAVEU OPERATIONS IN THE UNITED STATES, 1989 (OPERATOR DATA)

1. General statistics on injury experience and worktime-_-

2. Injưry experience by degree and worktime for work location and employment size group-- 13

3. Number of injuries, 10st workdays, and average severity by degree for work location and employment size group-a-n- 14

4. Injury experience by degree and worktime for work location and State-

5. Number of injuries, lost workdays, and average severity by degree for work 1ocation and State- 20

6. Number of injuries, incidence rates, and severity measures by degree for work location and part of body injured-a...- 25

7. Number of injuries, 1ost workdays, and average severity by degree for work location and part of body injured-

8. Number of injuries, incidence rates, and severity measures by degree for work location and nature of injury--

9. Number of injuries, 1ost workdays, and average severity by degree for work Iocation and nature of injury- -

10. Number of injuries, lost workdays, and average severity by degree for part of body injured and nature of injury-- -

11. Number of injuries by accident classification and degree

12. Number of lost workdays by accident classification and degree of injury for work 1ocation and Staie- 57

13. Number of injuries, 10st workdays, and average severity by degree for work location and occupation at time of injury---

14. Number of injuries, lost workdays, and average severity $w y$ degree for work location and accident classification-------

15. Number of injuries, 1ost wozkdays, and average severity by degree for accident classifiration and part of body injured--

16. Number of injuries, lost workdays, and average severity by degree for accident classification and nature of injury--...-

17. Occurrences reported to MSHA as occupational illnesses------ 
TABLES FOR SAND AND GRAVEL OPERATIONS IN THE UNITED STATES, 1989

(OPERATOR DATA)--Continued

13. Related noncoal mining summary reference tabulation, number

Page of injuries, incidence rates, average number of workers, and employee-hours by work location and mineral industry-----

19. Related coal mining summary reference tabulation, number of injuries, incidence rates, average number of workers. employee-hours, and production by type of coal mined and work locati n-.....

TABLES FOR SAND AND GRAVEL OPERATIONS IN THE UNITED STATES, 1989 (CONTRACTOR DATA)

Table reference guide (contractor data)- 92

1. General statistics on injury experience and severity-_an 94

5. Number of injuries, 10st workdays, and average severity

7. Number of injuries, lost workdays, and average severity by degree for work 1 ocation and part of body injured--

9. Number of injuries, lost workdays, and average severity by degree for work location and nature of injury- -

10. Number of injuries, $10 s t$ workdays, and average severity by degree for part of body and nature of injury-

umber of injuries by accident classification and degree

11. Number of injuries by accident classification and degre
for work location and State- Number of lost workdays by accident classification and degree of injury for work location and state-

13. Number of injuries, 10st workdays, and average severity by degree for work location and occupation at time of injury--.-

14. Number of injuries, 10st workdays, and average severity by degree for work location and accident classification-----

15. Number of injuries, 10st workdays, and average severity by degree for accident classification and part of body injured-

16. Number of injuries, lost workdays, and average severity by degree for accident classification and nature of injury-...--

17. Occurrences reported to MSHA as occupational illnesses----n-n--

18. Related nuncoal mining summary reference tabulation, number of injuries, incidence rates, average number of workers, and employee-hours by work location and mineral industry-...-. elated coal mining summary reference tabulation, number of injuries, incidence rates, average number of workers. employee-hours, and production by type of coal mined and work location- 
INJURY EXPERIENCE IN SAND AND GRAVEL MINING, 1989

by

Staff. Division of Mining Information Systems Safety and Health Technology Center

Technical Support, Denver, Colorado

\begin{abstract}
This Mine Safety and Health Administration (MSHA) informational report reviews in detail the occupational injury and illness experience of sand and gravel mining in the United States for 1989. Data reported by operators of mining establishments concerning work injuries are summarized by work location, accident classification, part of body jnjured, nature of injury, and occupation. Related inforuation on employment, worktime, and operating activity also is presented. Data reported by independent contractors performing certain work at mining locations are depicted separately in this report. For ease of comparison with other metal and nonmetallic mineral mining industries and with coal mining, summary reference tabulations are included at the end of both the operator and the contractor sections of this report.
\end{abstract}

\title{
INTRODU CTION
}

Data use in compiling this report were reported by operators of sand and gravel mining operations on a mandatory basis as required under the Federal Mine Safety and Health Act of 1977. Public Law 91-173 as amended by Law 95-164. Since January 1, 1978, operators of sand and gravel plants which are subject to the Act have been required under $30 \mathrm{CFR}$, Part 50, to submit reports of injuries, occupational illnesses, and related data.

Only data reported by the operators and contractors themselves axe utilized in this report. No estimates are made of employment, employeehours, or injuries for nonrespondent operations except as necessary to meet statistical processing requirements by providing minimum employment data for reported unmatched injury data.

Beginning with calendar year 1978, statistical measures for injury experience in mining were changed from the measures used from 1930 through 1977. The statistical measures for injury experience adopted by MSHA in 1978 compare closely with the measures used in the Office of Occupational Safety and Health Statistics, Bureau of Labor Statistics, U. S. Department of Labor. Therefore, beginning with $19 / 8$ data, the mining industry can be compared on a standard basis with other United States industries reporting under the Occupational Safety and Heal th Act of 1970.

Occurrences identified as occupational illnesses by mine operators were reported to MSHA and were processed in accordance with 30 CFR PART 50.20-6. The reported illnesses are summarized in seven categories for each work location and State. Incidence rates and severity measures are not computed for occupational illnesses. 
Because of the modifications in reporting and processing procedures that became effective January 1, 1978, injury rates as currently computed are not precisely comparable to those of previous years, except for fatality rates in which the "incidence rate" (the term used after 1977) is one-fifth of the "frequency rate" (the term used prior to 1978) for otherwise similar groupings of fatal injuries and worktimes. Because of this disparity, MSHA annual publications concerning injuries, illnesses, and operating activity will not contain data for years prior to 1978 .

\section{SCOPE OF STATISTICS}

The statistical data in this report cover the work experience of all personnel engaged in mine exploration, development, production, maintenance. repair, and construction work, including supervisory and technical personnel, working partners, and onsite officeworkers. Injury and illness data for all sand and gravel produced for unconsolidated deposits are covered in this report; no distinction is made between construction sand and industrial sand. Some industrial sands are produced from consolidated deposits that are classified by MSHA as stone operations because of the mining proculures used in extraction of the material.

It should be noted that amendments to legislation that funded MSHA and other government programs from December 16, 1981, through July 18, 1982, prohibited MSHA from administering or enforcing the Federal Mine Safety and Health Act of 1977 (Mine Act) or MSHA standards and regulations with respect to any person engaged in the surface mining or milling of stone, clay, feldspar, colloidal phosphate, or sand and gravel. They also provided that MSHA could not administer or enforce the Mine Act or MSHA standards and regulations on any State or political subdivision thereof. Further. MSHA could not administer or enforce the Mine Act or MSHA standarda or regulations with respect to any independent construction contractor who was engaged by an operator for the construction, repair or alteration of structures, facilities, utilities or private ways or roads located on (or appurtenant to) the surface areas of any coal or other mine, and whose employees worked in a specifically demarcated area, separate from actual mining or extraction activities. No data on exempted activities are included for that period of time in Table 1 of this publication.

MSHA issued guidelines which additionally reduced the reporting requirements of independent contractors. Reporting of employment, hours worked, and injury/illness data by independent contractors is 1 imited to the following types of work performed: mine development, including shaft and slope sinking; construction or reconstruction of mine facilities, including building or rebuilding preparation plants and mining equipment, and building additions to existing facilities; demolition of mine facilities: construction of dams; excavation or earthmoving activities involving mobile equipment; equipment installation, such as crushers and mills; equipment service or repair done underground or in the pit for a period exceeding five 
consecutive days at a particular mine; material handling within mine property, including haulage of coal, ore, refuse, etc., unless for the sole purpose of direct removal from or delivery to mine property; drilling and blasting. Also, data collected for employment and hours worked in those activities reflect the contractor's total metallic/nonmetallic work locations and total coal work locations and not the individual mine sites. For more detailed information concerning these changes, see the guidelines accompanying MSHA Policy Memoranda Nos. 82-6A MM and 81-35A C.

\section{DEFINITION OF TERMS}

Occupational Injury.--An occupational injury is an injury to a mine worker which occurs at a mine and for which medical treatment is administered, or which results in death or 1 oss of consciousness, restriction of work or motion, inability to perform all job duties on any day after an injury, 1ost workdays, temporary assignment to other duties, transfer to another job, or termination. The injury usually results from a recognizable single incident. For example, an explosion of a battery which splattered a worker with sulfuric acid would be a single incident and the worker would be considered to have suffered an injury. Another example would be an injury where a worker was overcome by hydrogen sulfide gas released from an exploding vessel.

Occupational illness.--An occupational illness is an illness or disease of a mine worker which may have resulted from work at a mine or for which an award of compensation is made. To be classified as an occupational illness, the disability must result from repeated exposure to the condition or substance which caused the disability. A classic illness example is a pneumoconiosis disability which may have been induced by repeated exposure to the causative dusty condition. Dermatitis resulting from repeated exposure to 1 ime dust or other material in the work environment also should be reported as an illness. In cases where the time of onset of illness is in doubt, the day of diagnosis of illness will be considered as the first day of illness.

Number of Operations and Associated Work Locations.--Number of operations is the number of individual respondents with a unique Federal identification number that have reported worktime data. The count and kind of operation are based on the 1 owest numbered (prinary) subunit on employment report Form 7000-2 (see figure 2, p. 10) that contains worktime data. An associated work location is a subunit reported with a primary subunit, e.g., an "office" is almost always an associated work location. Independent contractors working on mine property performing the types of work described under "Scope of Statistics" are assigned an identification number, report to MSHA, but are not included in the count of operations. Contractor data are depicted separately in this report.

Worktime.--Worktime includes the number of workers and the number of employee-hours worked. "Average number of workers" is a summary of the average number of persons working at individual establishments during calendar quarters of active operations. "Employee-hours" is a summary of employee-hours reported. 
Employment Size Group.--Reporting operations are classified into size groups according to the average number of workers in specified work locations.

Degree of Injury.--Degree of injury indicates the seriousness of injuries. FATAL injuries are those occurrences resulting in death, NFDL (NonFatal with Days Lost) injuries are nonfatal occurrences that result in days away from work, statutory days charged, or days of restricted work activity. NDL (No Days Lost) injuries are occurrences having no lost workdays; that is, nonfatal injury occurrences resulting only in temporary loss of consciousness or medical treatment other than first aid.

A permanent total disability is caused by any work injury or illness other than death that permanently and totally incapacitates a worker from following any gainful occupation, or that results in thi 1oss, or the complete 10ss of use, of both or any combination of : hands, arms, legs, feet, or eyes.

A permanent partial disability is caused by any work injury or iliness other than death or permanent total disability that results in the 1oss, or the complete loss of use, of any member or part of a member of the body, or any permanent impairment of functions of the body or part thereof, regardless of any preexisting disability of the injured member or impaired body function.

Incidence Rate.--Incits nce rate is defined as the number of injuries per 200,000 employee-hours, rounded to two decimal places. The highest rate given is 99.99. If an incidence rate is greater than 99.99 , then 99.99 is printed. If no data were reported, then a hyphen is printed. If a computation is less than 0.01 after rounding, then an asterisk inside parentheses $(*)$ is printed. The following is the standard incidence rate formula:

$$
I R=\frac{\text { number of injuries } \times 200,000}{\text { number of employee-hours }}
$$

Lost Workdays (LWD).--Lost workdays consist of days awa' from work (DAW) and days of restricted work activity (DRA), or statutory days charged as prescribed from a table of standard charges (see p. 7), e.1.., 6,000 days for a fatality or permanent total disability.

Severity Measure.--Severity measure is the number of lost workdays per 200,000 employee-hours, rounded to whole numbers. The highest rate given is 99.999. If the computed rate is greater than 99,999 , then 99,999 is printed. If no data were reported, then a hyphen is printed. If the computation reaults in a zero after rounding, then an asterisk inside parentheses ( $*$ ) is printed. The following is the standard severity measure formula:

$$
S M=\frac{\text { number of lost workdays } \times 200,000}{\text { number of employee-hours }}
$$


Average Severity.--Average severity is the average number of 1ost workdays per injury and is computed only for injuries classified as FATAL or NFDL. Lost workdays are selected on the same basis as that for computing the severity measure. The following is the standard average severity formula:

$$
\text { AS }=\frac{\text { number of 1ost workdays }}{\text { number of injuries contributing the 1ost workdays }}
$$

Classification of Accident.--Accident classification identifies circ mstances which contributed most directly to the resulting accident. The 21 classifications in the following 1 ist are designed to categorize all accidents, whether injury-producing or not.

Electrical.--Accidents in which the electric current is most directly responsible for the resulting accident.

Entrapment.--Accidents involving entrapment of persons.

Exploding Vesse1s Under Pressure.---Accidents involved with bursting of air hoses, air tanks, hydraulic 1 ines, hydraulic hoses, stand pipes, etc., due to internal pressure.

Explosives and Breaking Agents.--Accidents involving the detonation of manufactured explosives; includes Airdox and Cardox.

Falling, Rolling, or Sliding Rock or Material of Any Kind.-Accidents caused directly by falling material. other than material from the roof or face. Or, if material was set in motion by machinery, by haulage, by handtools, or while being handled or disturbed, etc., the force that set the material in motion determines the classification. For example, where a rock was pushed over a highwall by a bulldozer and the rock hit another rock which hit and injured a worker--the accident is classified as machinery. Machinery (a bulldozer) most directly caused the resulting accident.

Fall of Face, Rib, Pillar, Side, or Highwal1 (from in place).-Accidents in this classification include falls of material while barring down or placing props, also pressure bumps and bursts. Not included are accidents in which the motion of machinery or haulage equipment caused the fall either directly or by knocking out support.

Eall of Roof, Back, or Brow (from in place).--Underground only Accidents which include falls while barring down or placing props, also pressure bumps and bursts. Not included are accidents in which the motion of machinery or haulage equipinent caused the fall either directly or by knocking out support. 
Fire.--Accidents related to uncontrolled burning of material or mineral in the mine environment. Not included are fires initiated by electricity or by ignition or explosion of gas or dust.

Handling Material.--Accidents related to handling packaged or loose material while lifting, pulling, pushing, or shoveling.

Handtools.--Accidents related to nonpowered tools.

Nonpowered Haulage.--Accidents related to the motion of nonpowered haulage equipment. Included are accidents involving wheelbarrows, manually pushed mine cars, timber trucks, etc.

Powered Haulage.--Accidents related to the motion of powered haulage equipment. Included are accidents involving conveyors, front-end loaders, forklifts, shuttle cars, load-haul-dump units, locomotives, railroad cars, haulage trucks, pickups, automobiles, and personnel carriers.

Hoisting.--Accidents involving cages, skips, ore buckets, and elevators. The accident results from the action, motion, or failure of the hoisting equipment or mechanism. Included are equipment such as cranes and derricks only when used in shaft sinking; also suspended work platforms in shafts. Not included is equipment such as chain hoists, come-alongs, and winches.

Ignition or Explosion of Gas or Dust.--Accidents resulting as a consequence of the ignition or explosion of gas or dust.

Impoundment.--Accidents caused by an unstable condition or failure of an impoundment, refuse pile, or culm bank requiring emergency preventative action or evacuation of an area.

Inundation.---Accidents caused by inundation of a surface or underground mine by a liquid (or semi-solid) or a gas.

Machinery.--Accidents related to the motion of machinery. Included are all electric and air-powered tools and mining machinery such as drills, tuggers, winches, slushers, draglines, power shovels, loaders, and compressors.

S1if or Fal1 of Person (from an el evation or on the same 1eve1).-Accidente include slips or falls while getting on or of machinery and haulage equipment which is not moving, and slips or falls while servicing or repairing equipment or machinery.

Stepping or Kneeling on Object.--Accidents are classified in this category only where the object stepped or kneeled on contributed most directly to the accident.

Striking or Bumping.-- This classification is restricted to those accidents in which an individual, while moving about. strikes or bumps an object but is not handling material, using handtools, or operating equipment. 


\section{Other.--Accidents not elsewhere classified.}

Abbreviations.--Space limitalions in column headings and stub entries of standard-format tabulations require occasional adaptation of terminology. In this report PLNTS should be interpreted as "plants."

Statutory Days Charged.--Some injuries or illnesses are of such a degree of severity that a standard time charge of $10 s t$ workdays has been adopted by MSHA as follows:

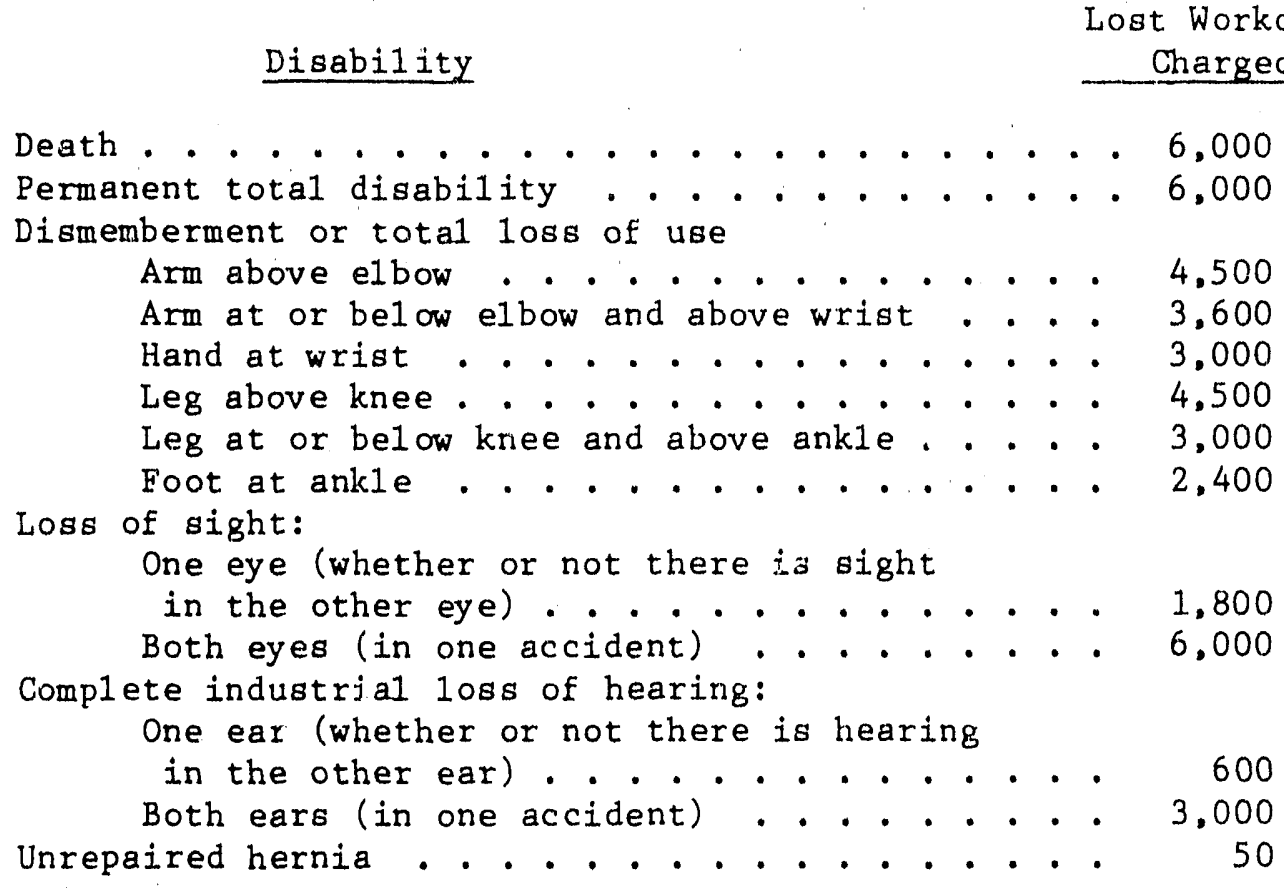

2/ Not more than 6,000 lost workdays will be charged in a single accident that results in multiple injuries to a mine worker. (See definition of permanent total disability on page 4. )

\section{TABLE REFERENCE GUIDE (OPERATOR DATA)}

The following reference guide facilitates locating particular data elements contained in the annual statistical tables for operators. A comparable guide precedes the contractor section of this report. The number appearing in each cell indicates the table number in which that particular element may be found. All elements listed under the stub headings appear as vertical headings in the tables. Elements listed under column headings appear as horizontal headings. The only exception to this is table 1, in which the headings have been reversed.

To use this guide, read lown or across the elements listed in both the stub and column headings to detennine which combinations are of interest. For example, incidence rates by work location appear in six different tables, numbers $2,4,6,8,18$, and 19 . However, only one, table 8 , provided a further division by nature of injury as wel.1. The occuparion of the injured is found in table 13 and, as seen in the column headings" provides the number of injuries, 10 st workdays, and average severity. 
REFERENCE GUIDE TO SAND AND GRAVEL STATISTICAL TABLES (OPERATOR DATA)

\begin{tabular}{|c|c|c|c|c|c|c|c|c|c|c|c|c|c|}
\hline sTuB aradings & 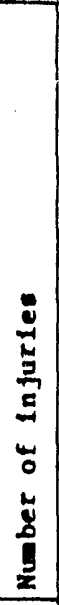 & 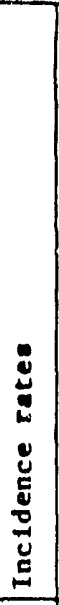 & 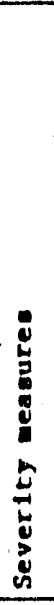 & $\begin{array}{l}0 \\
0 \\
0 \\
0 \\
3 \\
3 \\
0 \\
0 \\
0 \\
\end{array}$ & 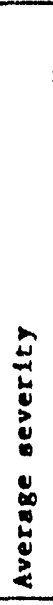 & 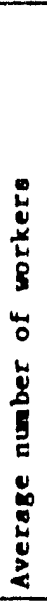 & 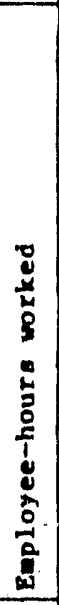 & 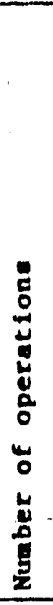 & 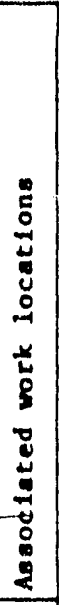 & 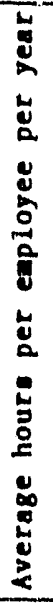 & 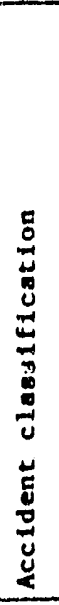 & 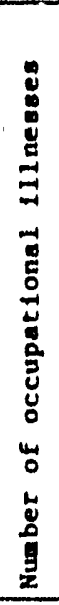 & 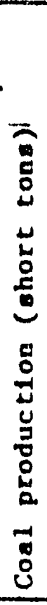 \\
\hline $\begin{array}{l}\text { Prior year data } \\
\text { with and without office }\end{array}$ & 1 & 1 & 1 & & 1 & 1 & 1 & 1 & 1 & 1 & & & \\
\hline \multirow{15}{*}{ Hork locat1on } & 2 & 2 & 2 & & & 2 & 2 & 2 & 2 & & & & \\
\hline & 3 & & & 3 & 3 & & & & & & & & \\
\hline & 4 & 4 & 4 & & & 4 & 4 & 4 & 4 & & & & \\
\hline & 5 & & & 5 & 5 & & & & & & & & \\
\hline & 6 & 6 & 6 & & & & & & & & & & \\
\hline & 7 & & & 7 & 7 & & & & & & & & \\
\hline & 8 & 8 & 8 & & & & & & & & & & \\
\hline & 9 & & & 9 & 9 & & & & & & & & \\
\hline & 11 & & & & & & & & 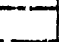 & & II & & \\
\hline & & & & 12 & & & & & - & & 12 & & \\
\hline & 13 & & & 13 & 13 & & & & & & & & \\
\hline & 14 & & & 14 & 14 & & & & & & & & \\
\hline & & & & & & & & & & & & 17 & \\
\hline & 18 & 18 & & & & 18 & 18 & & & & & & \\
\hline & 19 & 19 & & & & 19 & 19 & & & & & & 19 \\
\hline \multirow{2}{*}{ Eaployment ste group } & 2 & 2 & 2 & & & 2 & 2 & 2 & 2 & & & & \\
\hline & 3 & & & 3 & 3 & & & & & & & & \\
\hline \multirow{5}{*}{ State } & 4 & 4 & 4 & & & $\zeta$ & 4 & 4 & 4 & & & & \\
\hline & 5 & & & 5 & 5 & & & & & & & & \\
\hline & 11 & & & & & & & & & & 11 & & \\
\hline & & & & 12 & & & & & & & 12 & & \\
\hline & & & & & & & & & & & & 17 & \\
\hline \multirow{4}{*}{ Part of body Infured } & 6 & 6 & 6 & & & & & & & & & & \\
\hline & 7 & & & 7 & 7 & & & & & & & & \\
\hline & 10 & & & 10 & 10 & & & & & & & & \\
\hline & 25 & & & 15 & 15 & & & & & & & & \\
\hline \multirow{4}{*}{ Neture of Injury } & 8 & 8 & 8 & & & & & & & & & & \\
\hline & 9 & & & 9 & 9 & & & & & & & & \\
\hline & 10 & & & 10 & 10 & & & & & & & & \\
\hline & 16 & & & 16 & 16 & & & & & & & & \\
\hline $\begin{array}{l}\text { Occupation at the of } \\
\text { 2ajury }\end{array}$ & 13 & & & 13 & 13 & & & & & & & & \\
\hline \multirow{3}{*}{ Accldent clanolfication } & 24 & & & 16 & 14 & & & & & & & & \\
\hline & 15 & & & 15 & 15 & & & & & & & & \\
\hline & 16 & & & 16 & 16 & & & & & & & & \\
\hline \multirow{2}{*}{ Materal Induetry } & & & & & & & & & & & & 17 & \\
\hline & 18 & 18 & & & & 18 & 18 & & & & & & \\
\hline Type of conl & 19 & 19 & & & & 19 & 19 & & & & & & \pm 9 \\
\hline
\end{tabular}

I/ For table 1 ooly, the hasdinge have been reversed to elaplify this culde; that 10, the data elemente lodicated is the colime heading for this table actually appear as rertical heudings. 

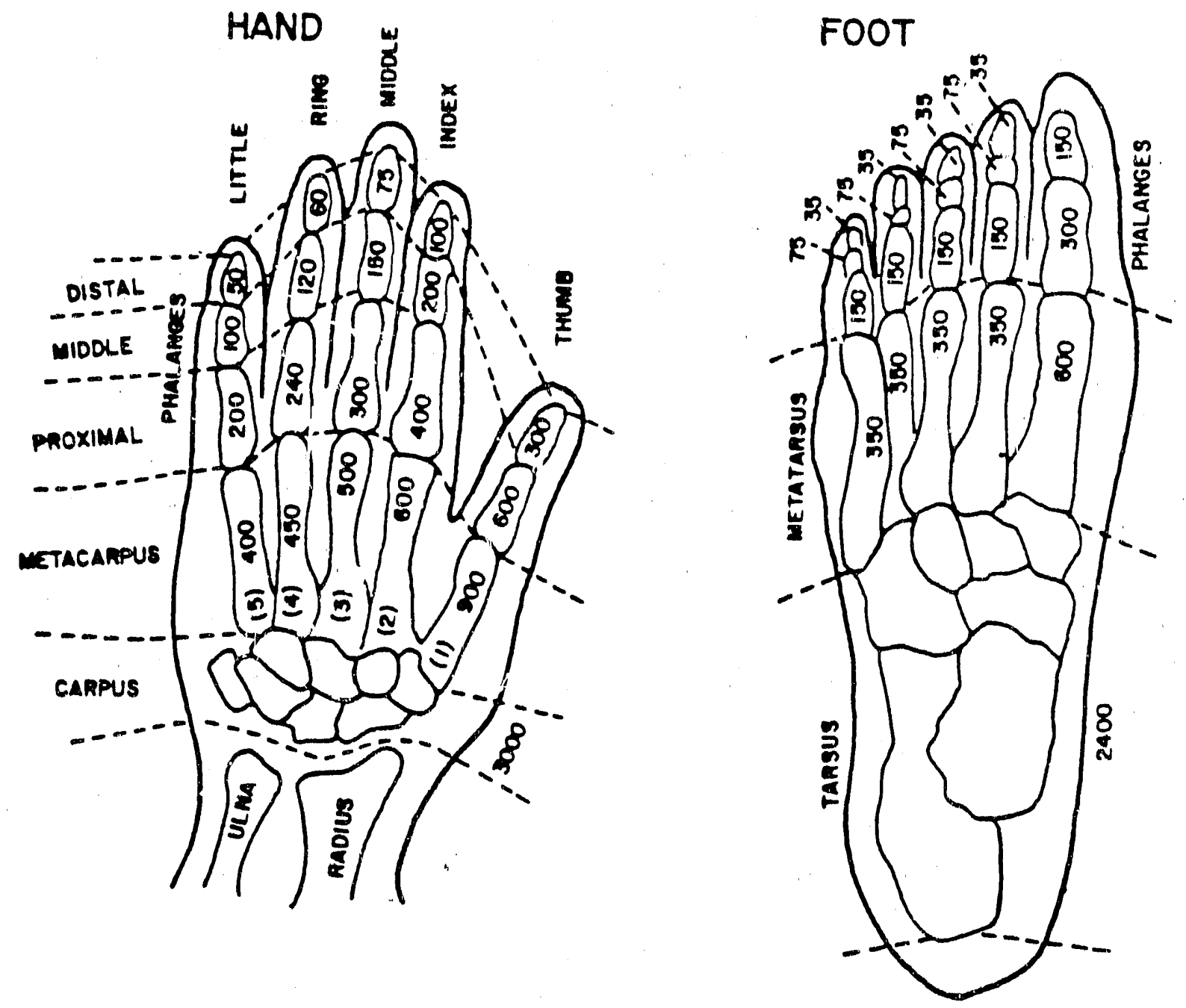

Note:

Numbers shown on diagrams are statutory charges of lost workdays for loss of all or part of bone.

Proportional charges may be cainuted based on medical determination of percentage loss of use.

FIGURE I. Scheduled charges in lost workdays for loss or bss of use involving hand or foot. 


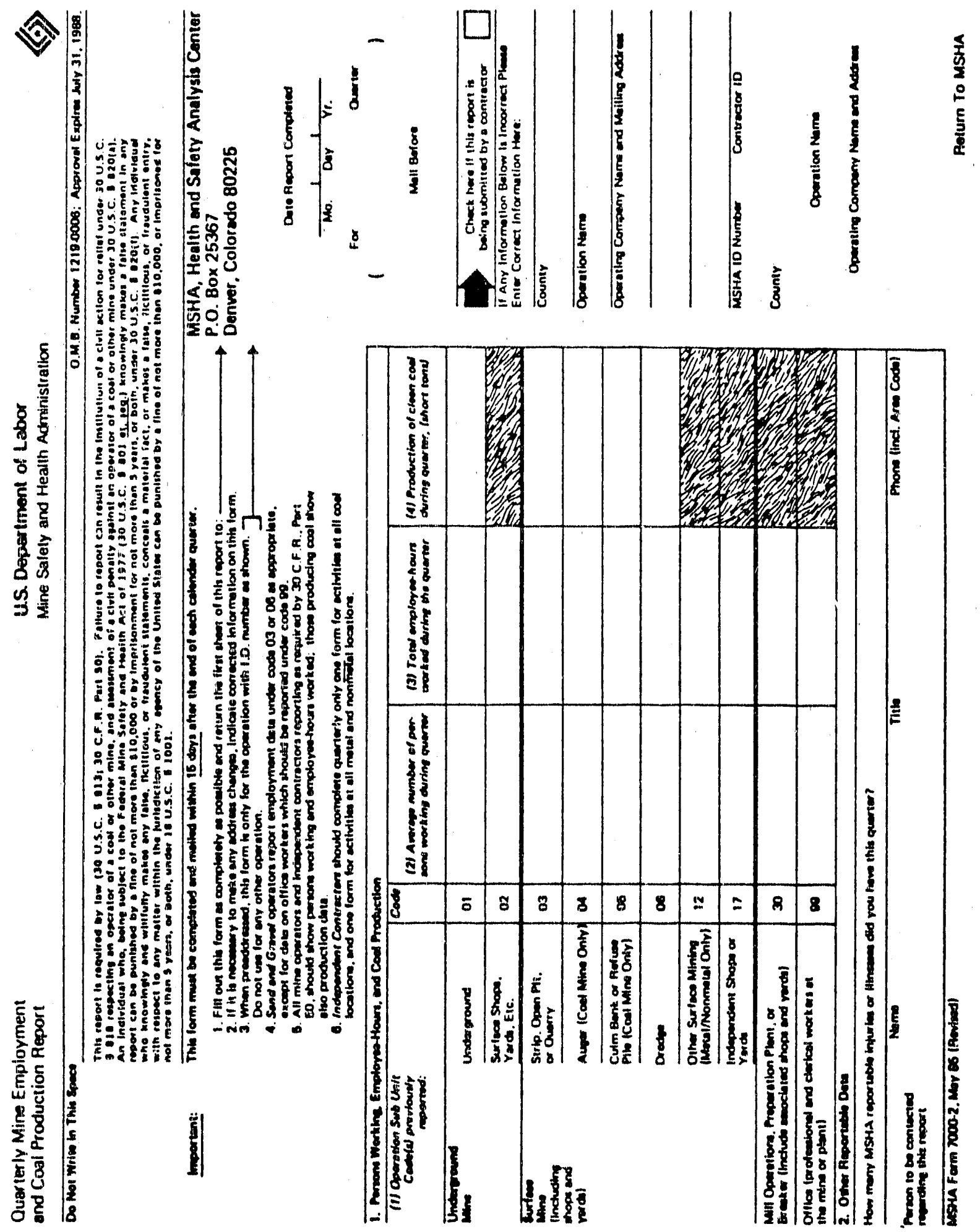

FIGURE 2, Quarterly mine employment and coal production report form. 
Mine Accident, Injury and IIIness Report

US. Department of Libor

Mine Safety and Health Adrmistration

Section A Jdemtificroon Dro

\begin{tabular}{|c|c|c|c|c|}
\hline MESHA IO NUMD & Contrector 10 & 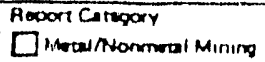 & $\square$ Cosl Mining & Doheck hore if resoort \\
\hline
\end{tabular}

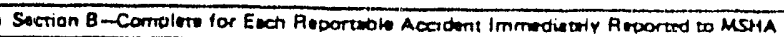

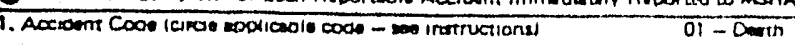

O4 - imunctation

05 -. Ses or Durt Ignition

QS - Mirm Fir

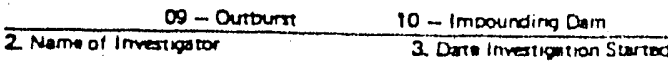

11 - Hoirting

02-Serioun injury

07 - Explosin

12 .. Othite Inpury
Co-Errtraprnent

$\infty$ - Acol Fall Mtortir Day Year

Sectoon C-Completw far Emch Feportaolo Accodent, Injury or llinexs

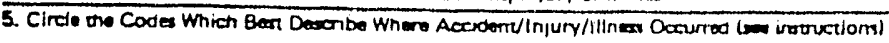

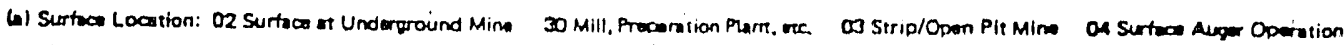

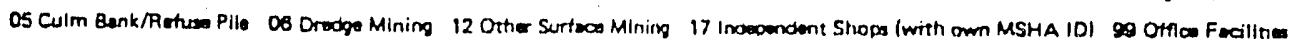

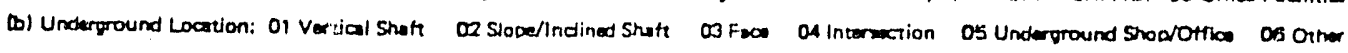

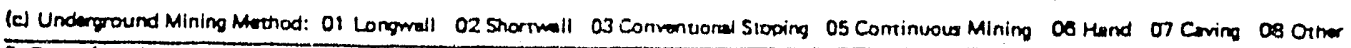

B. Dere of Accident

\begin{tabular}{|l|l|lll}
\hline Momh & Day & Yar & $\square \mathrm{mm}$ & $\square \mathrm{mm}$ \\
& & $\square \mathrm{mm}$ & $\square \mathrm{pm}$
\end{tabular}

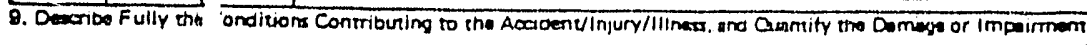

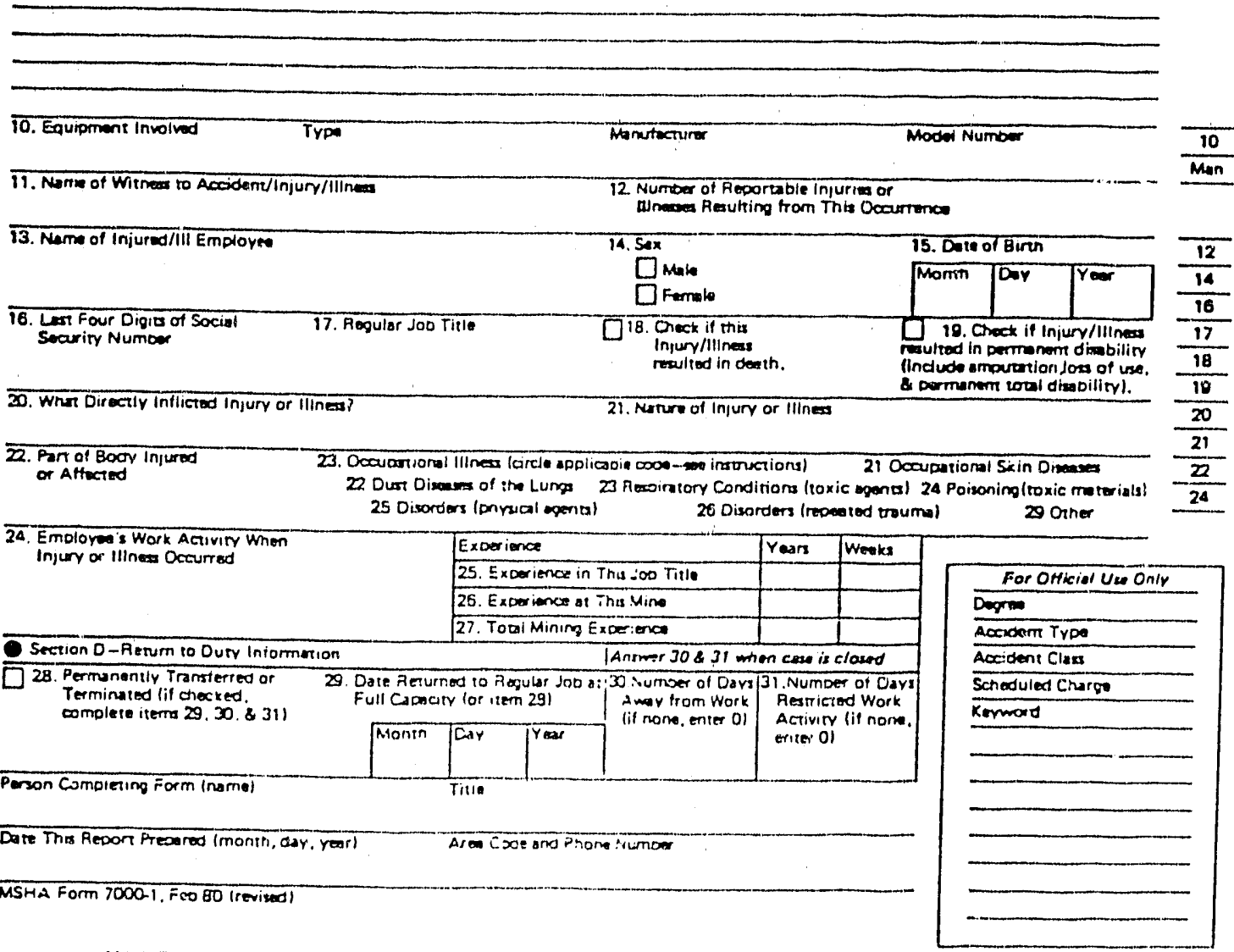

MAIL THIS PAGE TO THE HEALTH AND SAFETY ANALYSIS CENTER, MINE SAFETY

AND HEALTH ADMINISTRATION, P.O. BOX $: 36 \%$, DE.NVEFT, COLORADO 80225

FIGURE 3. Mine accident, injury, and illness report form. 


\begin{tabular}{|c|c|c|c|c|c|c|c|c|}
\hline \multirow{5}{*}{ 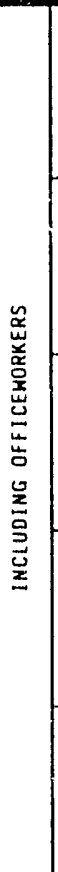 } & $\stackrel{\cong}{\Xi}$ & m8\% & & 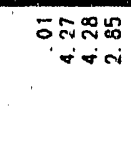 & $\frac{9}{2}$ & $\bar{n} \mathfrak{n} \approx$ & 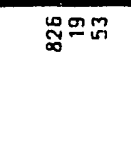 & 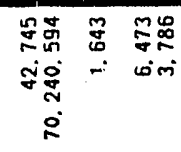 \\
\hline & 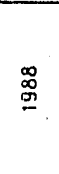 & 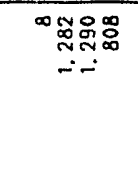 & $\begin{array}{l}\infty \\
0 \\
0 \\
i \\
i\end{array}$ & 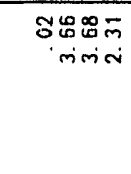 & $\begin{array}{l}\text { g } \\
\text { ज' } \\
\text { ज' }\end{array}$ & 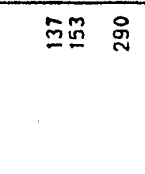 & ळ๐్ & 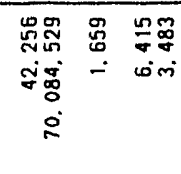 \\
\hline & $\stackrel{\infty}{\infty}$ & 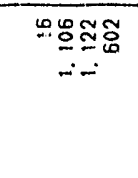 & 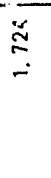 & $\begin{array}{l}\text { gNain } \\
\text { mimí }\end{array}$ & סे & 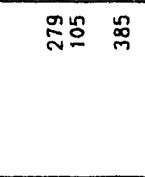 & 갱ㅁㅇㅛ & 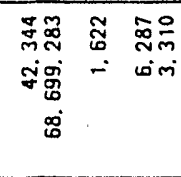 \\
\hline & 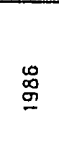 & 商品品 & 离 & 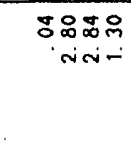 & $\ddot{\square}$ & 总的总 & 諰ন & 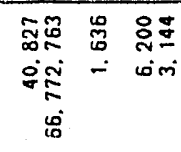 \\
\hline & 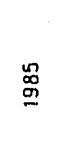 & 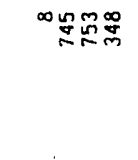 & $\stackrel{\square}{\square}$ & m్d & $\underset{\square}{n}$ & 踏怘 & 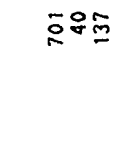 & 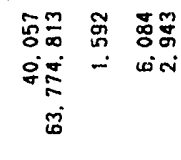 \\
\hline \multirow{5}{*}{ 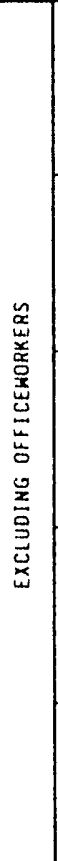 } & $\stackrel{\mathscr{\Xi}}{\stackrel{\Phi}{I}}$ & 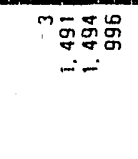 & $\begin{array}{l}\text { g } \\
\text { i }\end{array}$ & 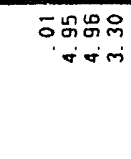 & $\underset{\infty}{\infty}$ & 造 总 & 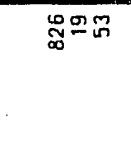 & 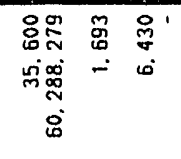 \\
\hline & 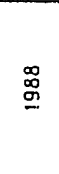 & 赵 & $\begin{array}{l}m \\
\stackrel{m}{0} \\
\stackrel{i}{N}\end{array}$ & 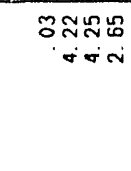 & $\begin{array}{l}8 \\
6 \\
6\end{array}$ & g్ & 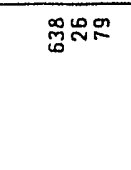 & 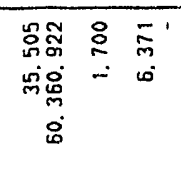 \\
\hline & $\stackrel{\tilde{\Phi}}{\Phi}$ & 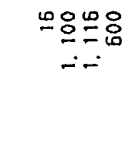 & $\stackrel{\circ}{\check{2}}$ & $\begin{array}{l}\text { SAREM } \\
\text { minin }\end{array}$ & क & స్లప & 跑の生 & 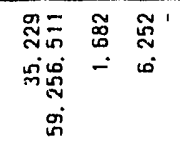 \\
\hline & 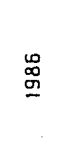 & 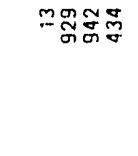 & $\stackrel{\substack{m \\
m}}{=}$ & 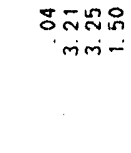 & $\stackrel{n}{g}$ & 용 \& & 음 $\cong$ & 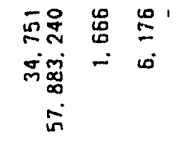 \\
\hline & 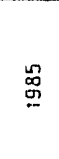 & 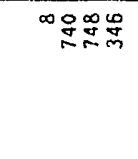 & : & 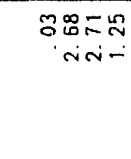 & 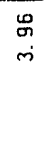 & 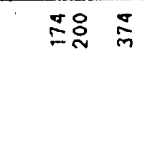 & ㅁㅇㅁㅇㅛ & 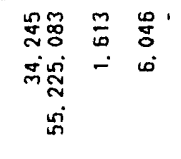 \\
\hline & & 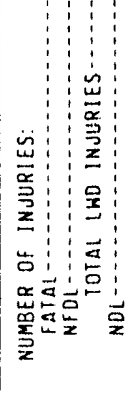 & 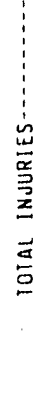 & 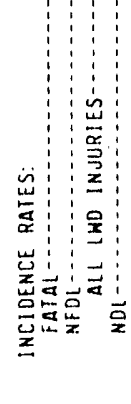 & 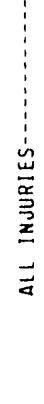 & 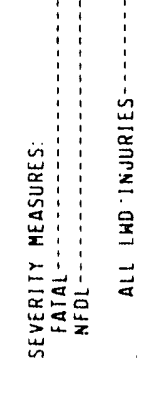 & 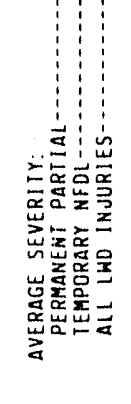 & 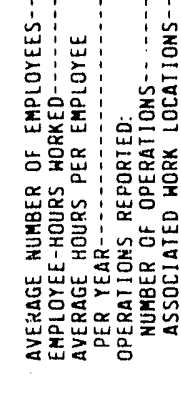 \\
\hline
\end{tabular}




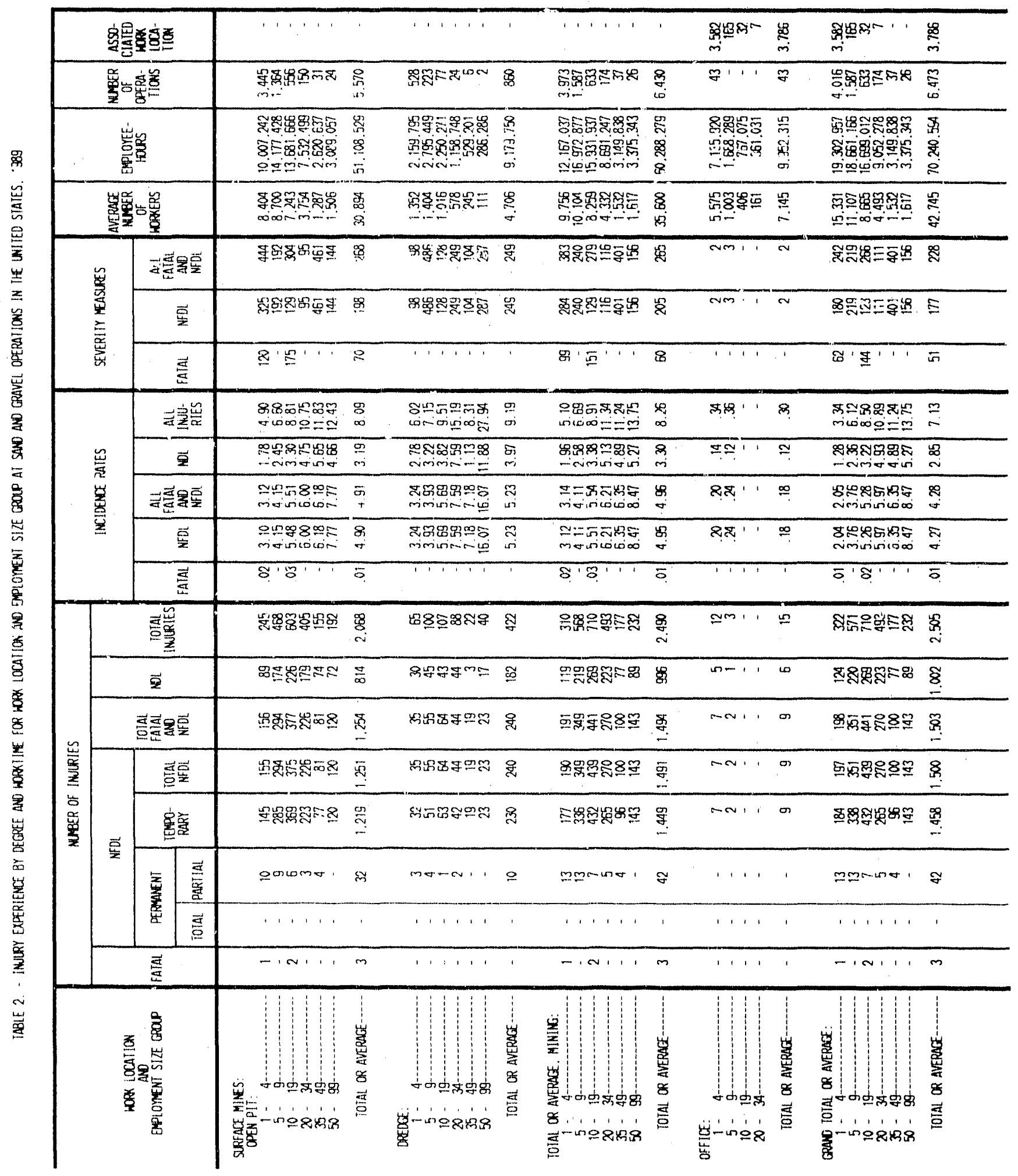




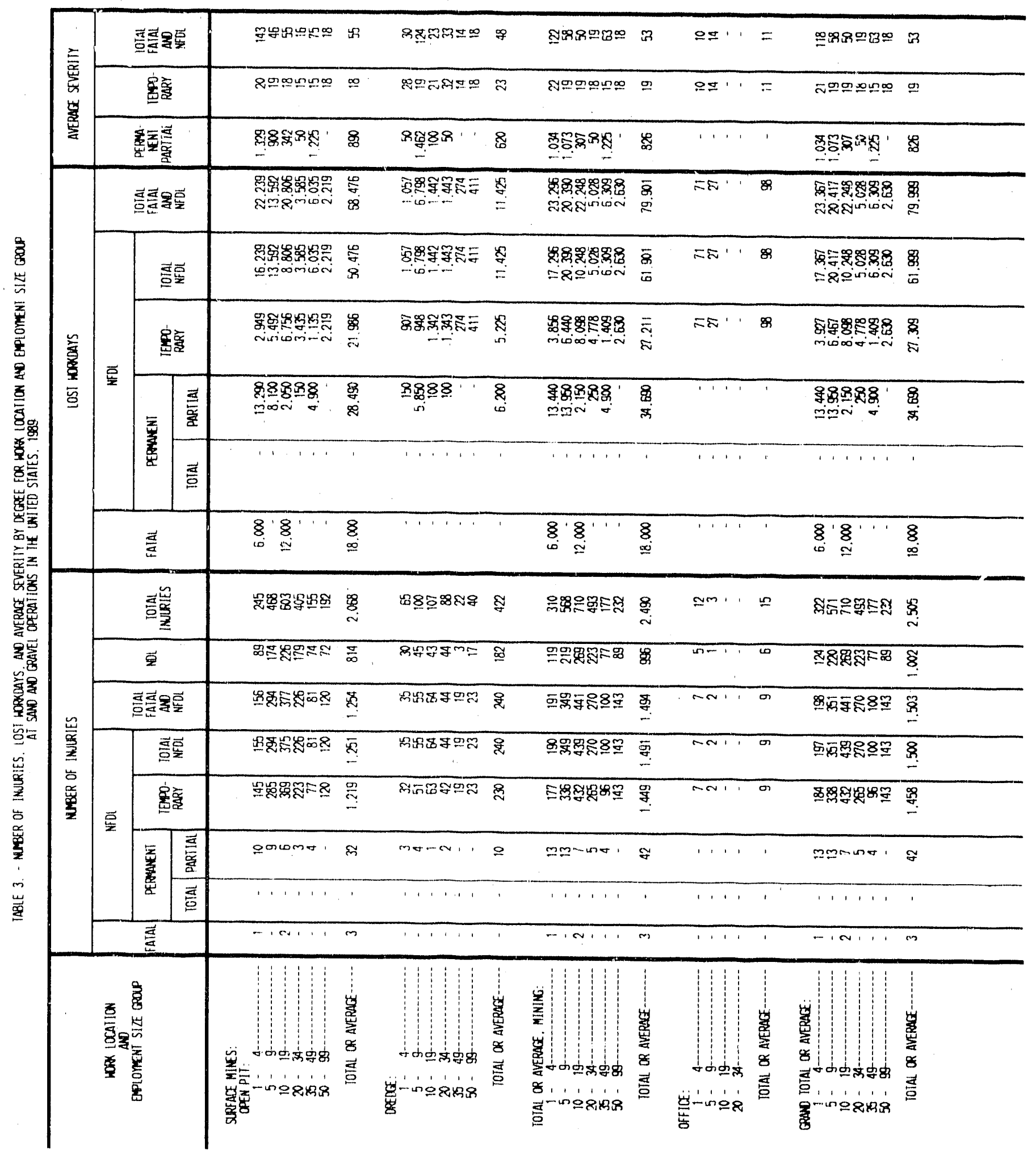




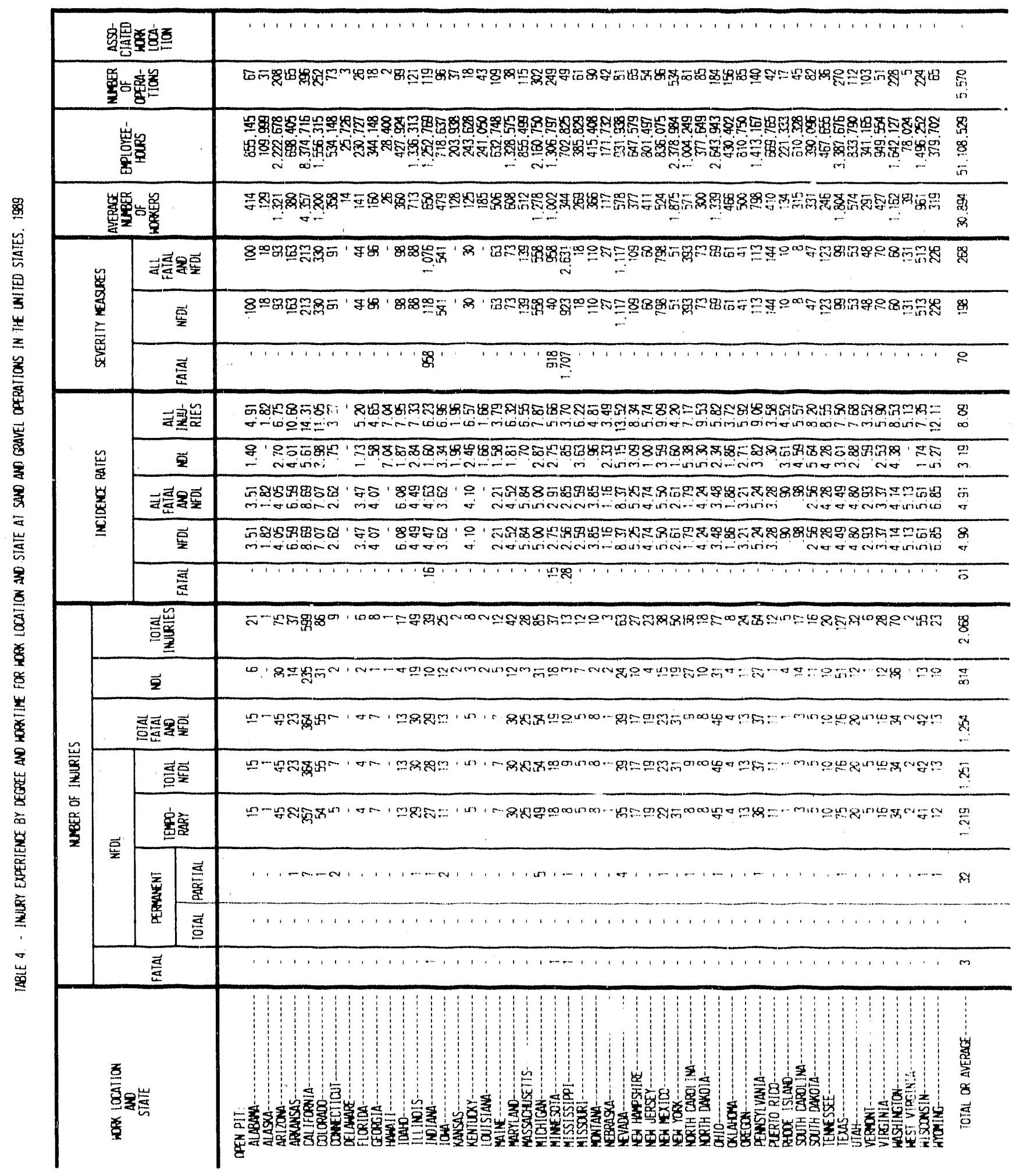




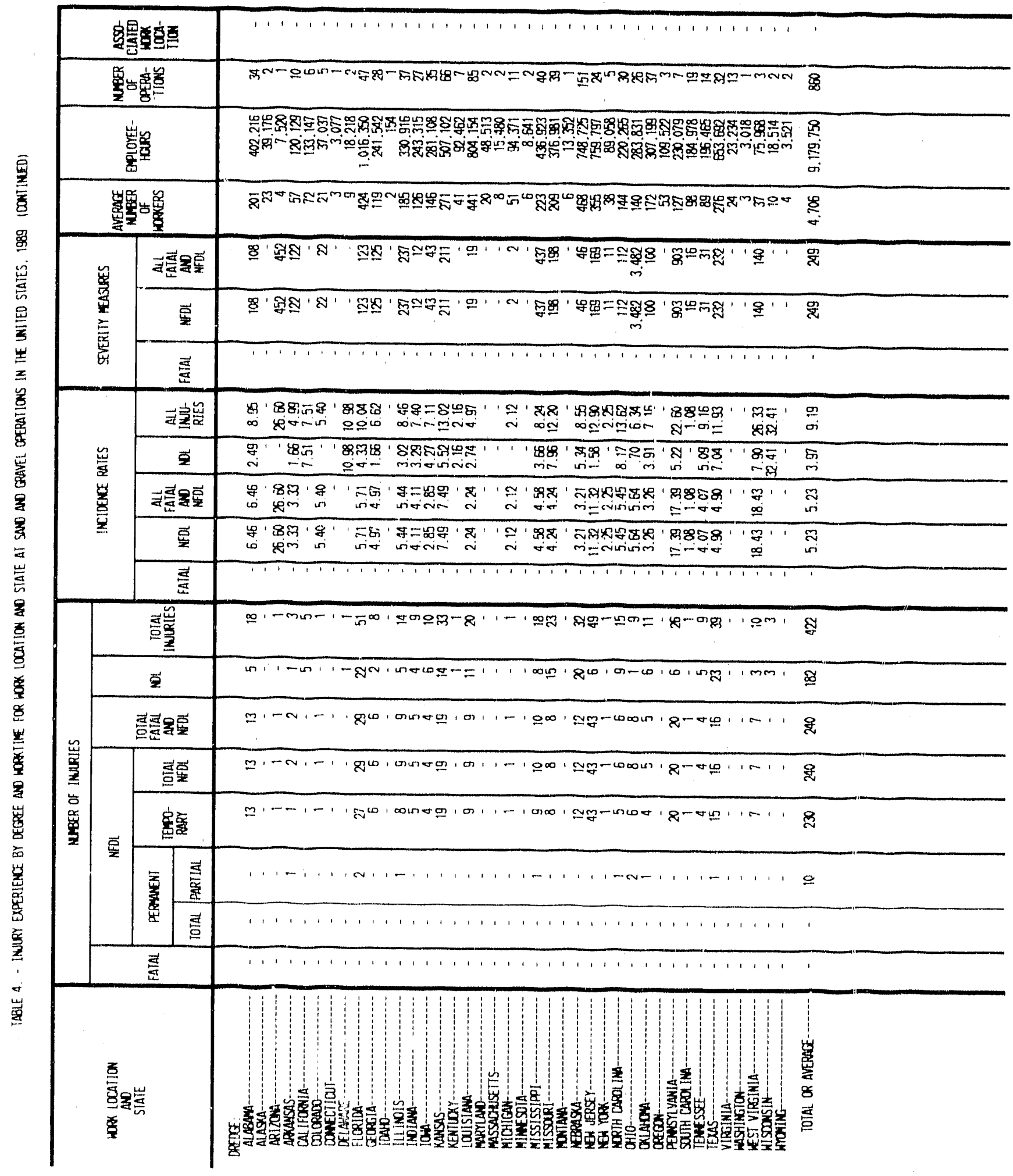




\begin{tabular}{|c|c|c|c|c|c|}
\hline \multicolumn{4}{|c|}{ 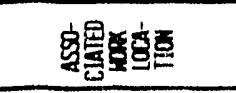 } & 1 & \\
\hline \multicolumn{4}{|c|}{ 受出音总 } & 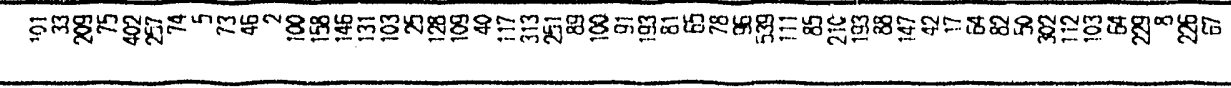 & $\underset{\substack{8 \\
0}}{\stackrel{8}{0}}$ \\
\hline \multicolumn{4}{|c|}{ 容害 } & 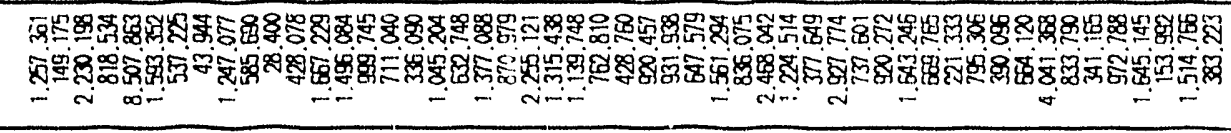 & 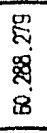 \\
\hline \multicolumn{4}{|c|}{ 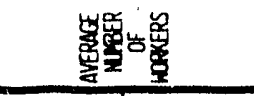 } & 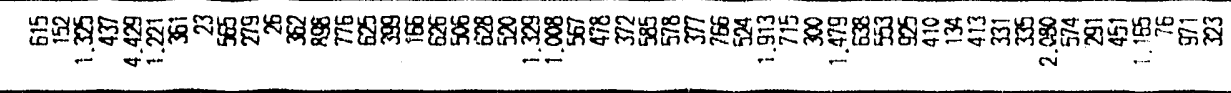 & $\begin{array}{l}8 \\
\text { si } \\
\end{array}$ \\
\hline \multirow{3}{*}{\multicolumn{2}{|c|}{ 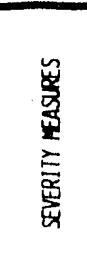 }} & \multicolumn{2}{|c|}{ 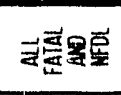 } & 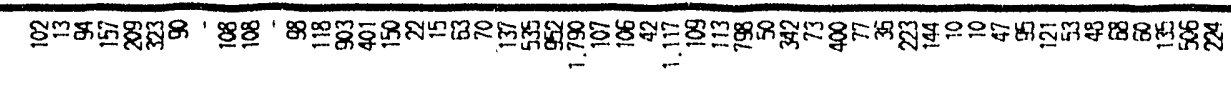 & 菓 \\
\hline & & \multicolumn{2}{|c|}{ 䓍 } & 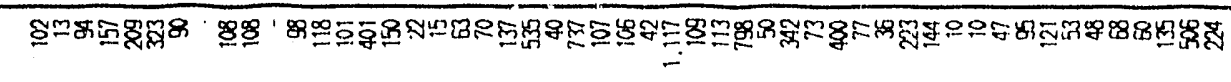 & 罗 \\
\hline & & \multicolumn{2}{|c|}{$\underset{z}{z}$} & 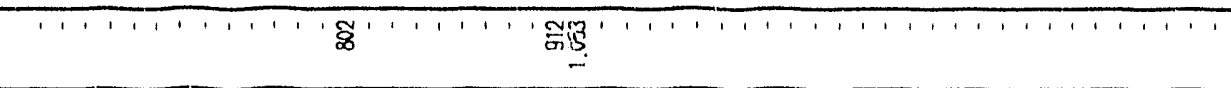 & $\bar{B}$ \\
\hline & \multirow{5}{*}{ 生 } & \multicolumn{2}{|c|}{ 当量岸 } & 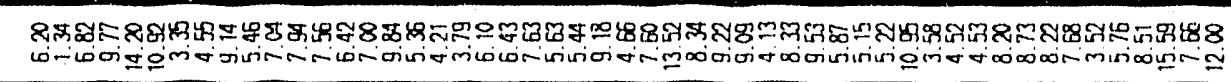 & $\underset{\infty}{\infty}$ \\
\hline & & \multicolumn{2}{|c|}{$\overrightarrow{\underline{2}}$} & 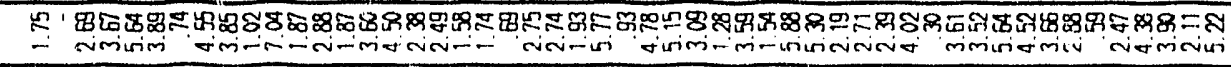 & $\stackrel{8}{m}$ \\
\hline & & \multicolumn{2}{|c|}{ 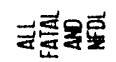 } & 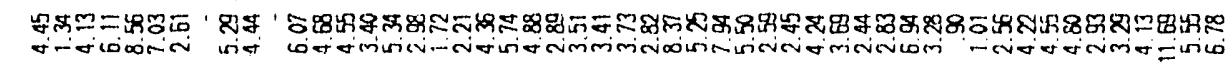 & $\underset{8}{8}$ \\
\hline & & \multicolumn{2}{|c|}{ 蔓 } & 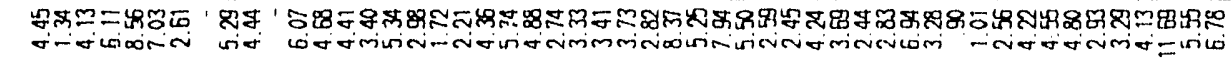 & gi \\
\hline & & \multicolumn{2}{|c|}{ 蛋 } & 표 & $\bar{a}$ \\
\hline \multirow{8}{*}{ 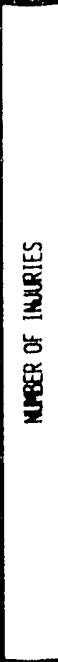 } & \multirow{2}{*}{\multicolumn{3}{|c|}{ 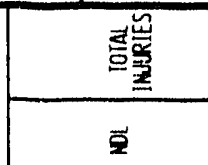 }} & 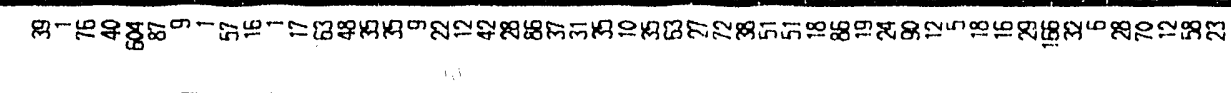 & $\underset{\substack{g \\
i}}{\sim}$ \\
\hline & & & & 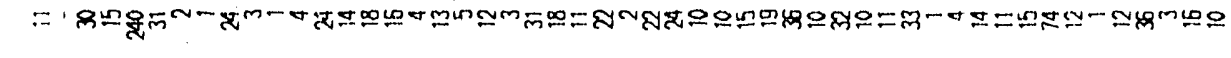 & 8 \\
\hline & \multicolumn{3}{|c|}{ 总起是要 } & 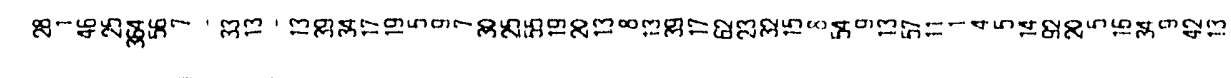 & 要 \\
\hline & \multirow{4}{*}{ 覇 } & \multicolumn{2}{|c|}{ 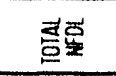 } & Q⿱宀 & 霖 \\
\hline & & \multicolumn{2}{|c|}{ 容言 } & 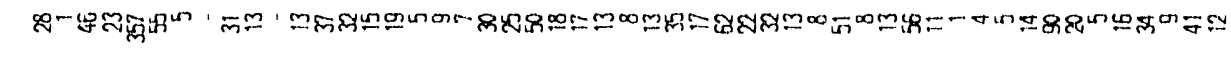 & 㝵 \\
\hline & & \multirow{2}{*}{ 淁 } & 章 & $-10 \mathrm{din}-1,-1,1, \mathrm{~N}_{1}, 1$ & 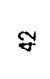 \\
\hline & & & 총 & $1,1,1,1$ & , \\
\hline & \multicolumn{3}{|c|}{ 妾 } & $\ldots$, & $m$ \\
\hline & \multicolumn{3}{|c|}{ 要㙏 } & 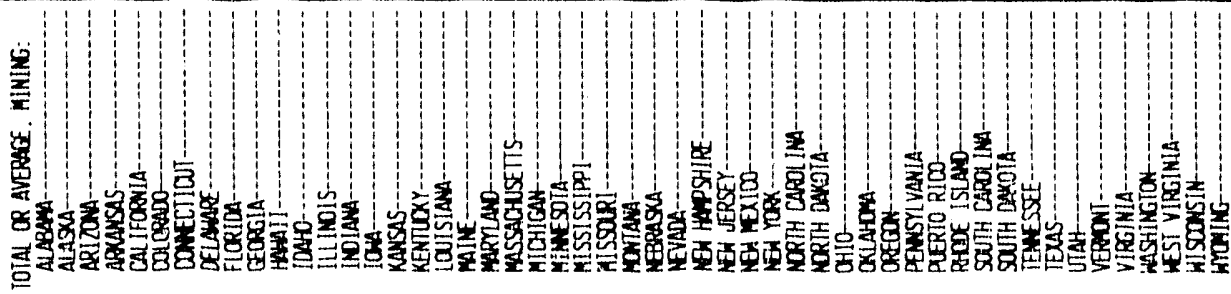 & 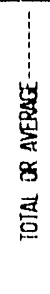 \\
\hline
\end{tabular}




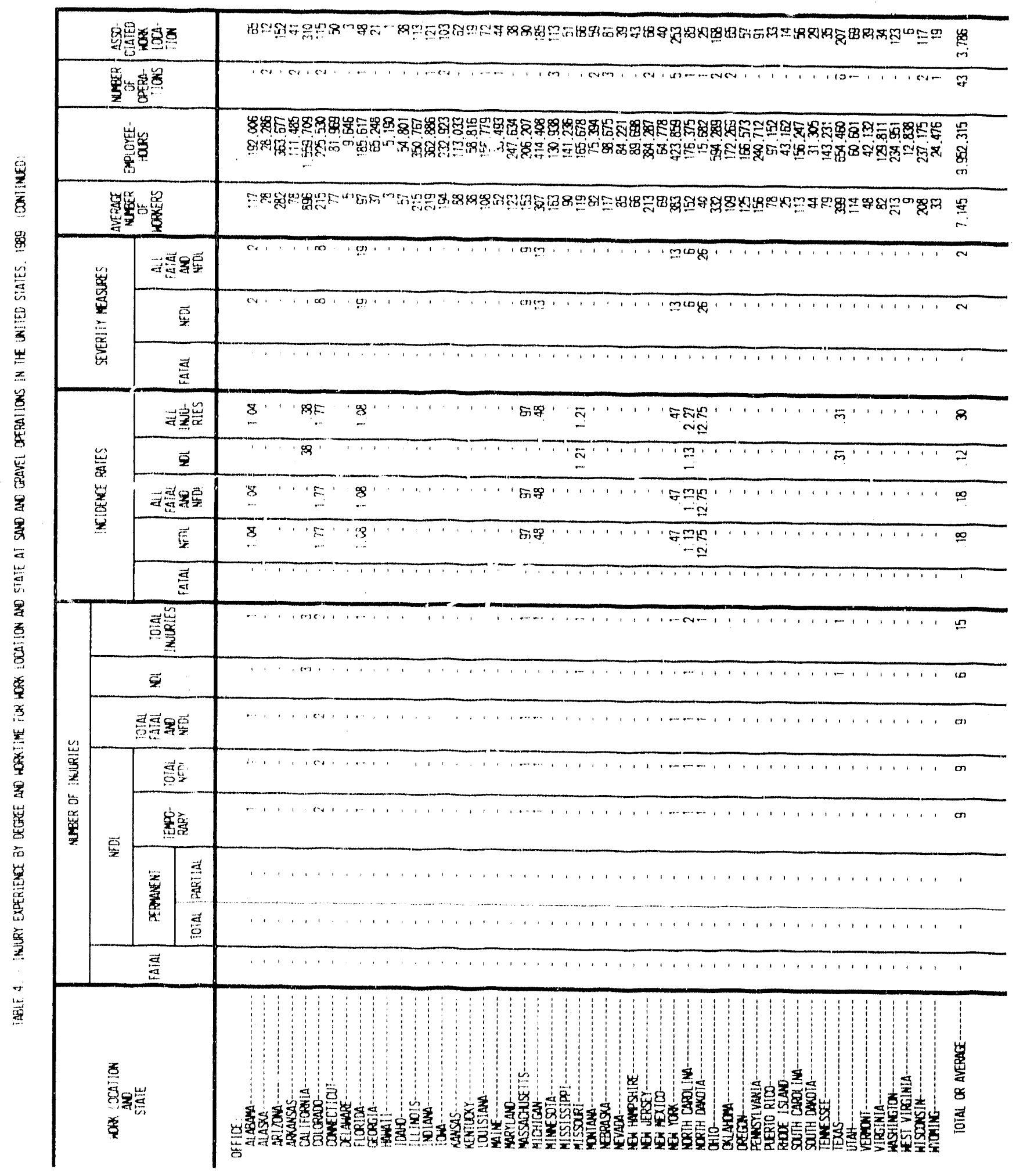




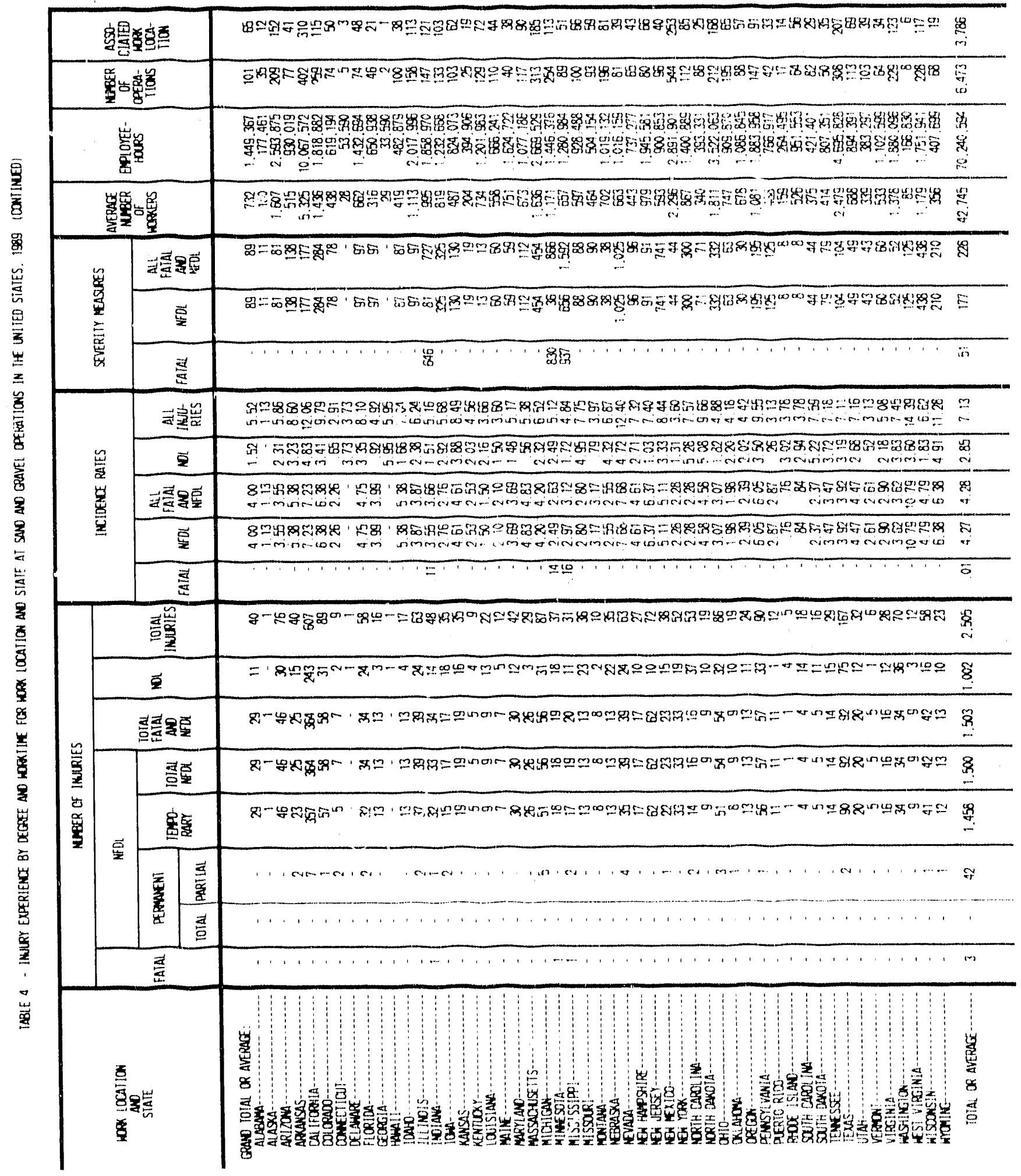




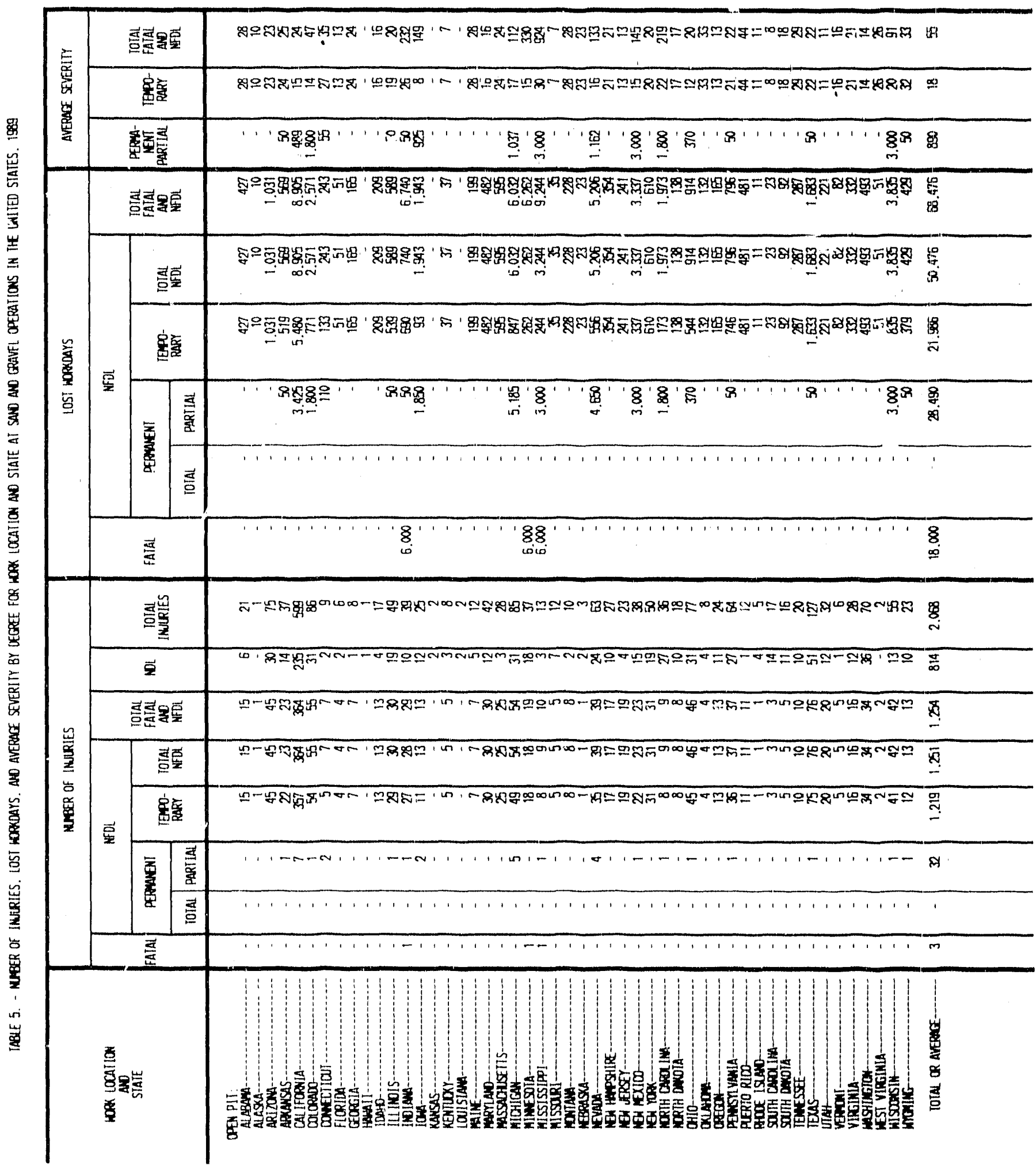




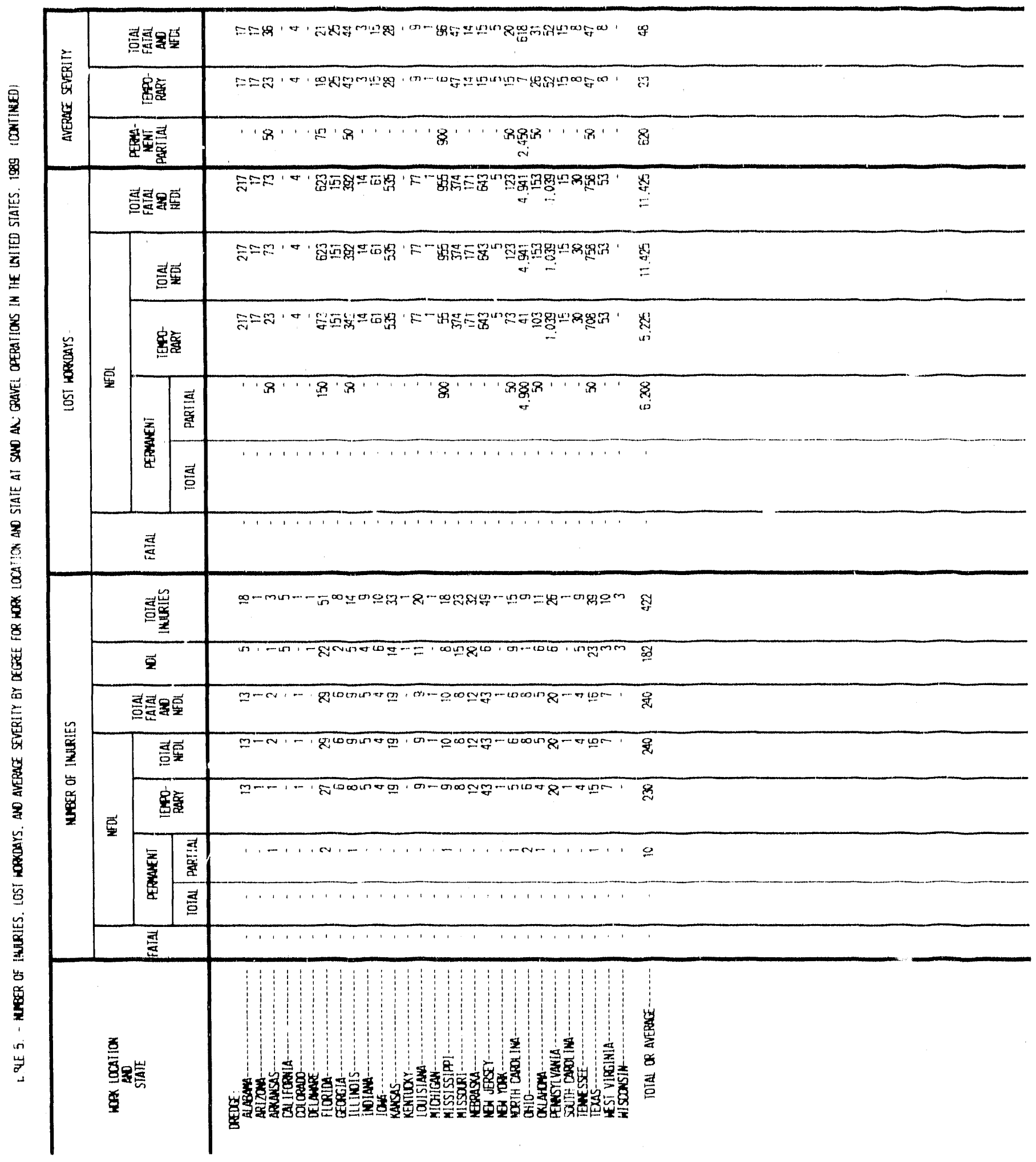




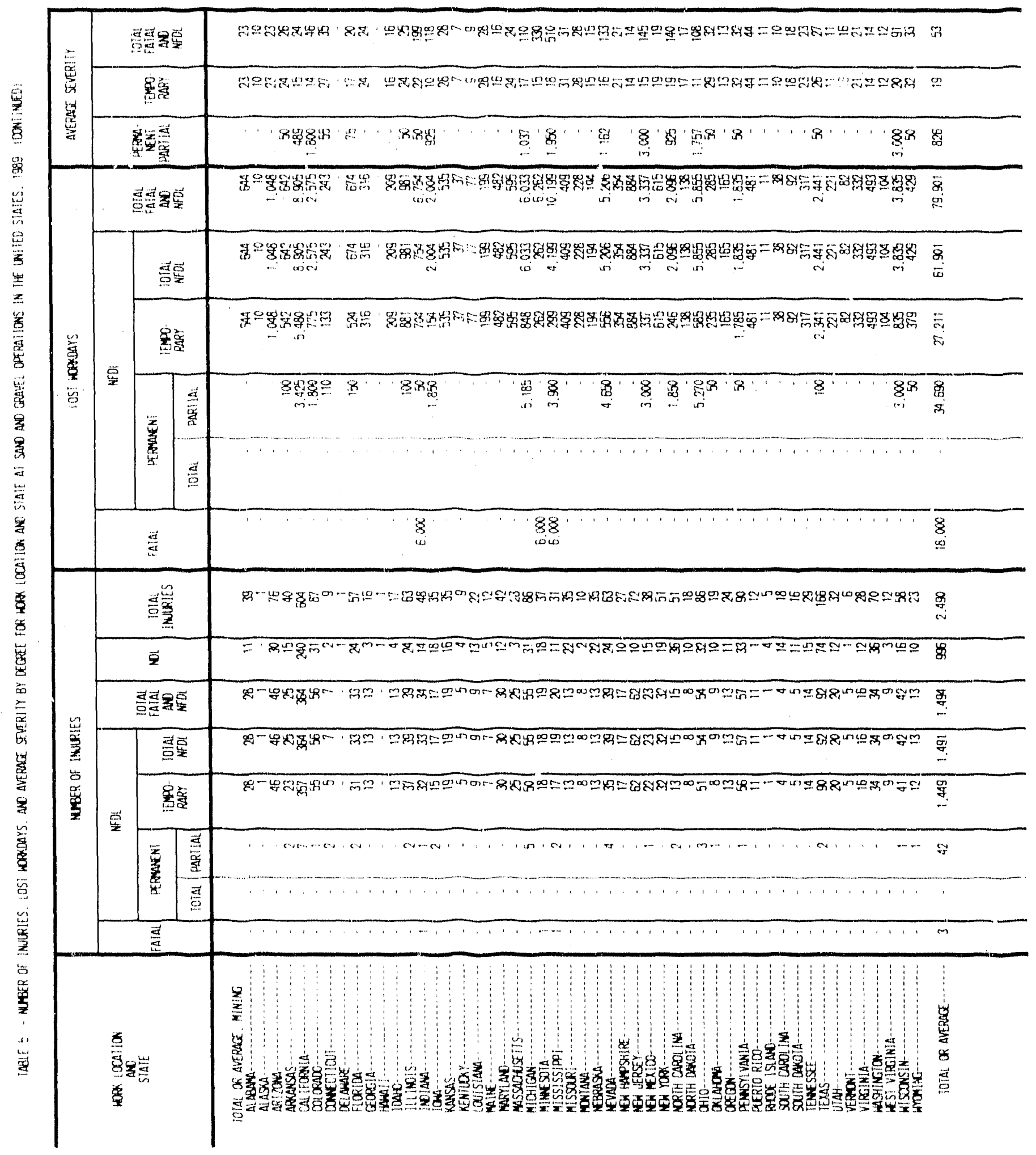




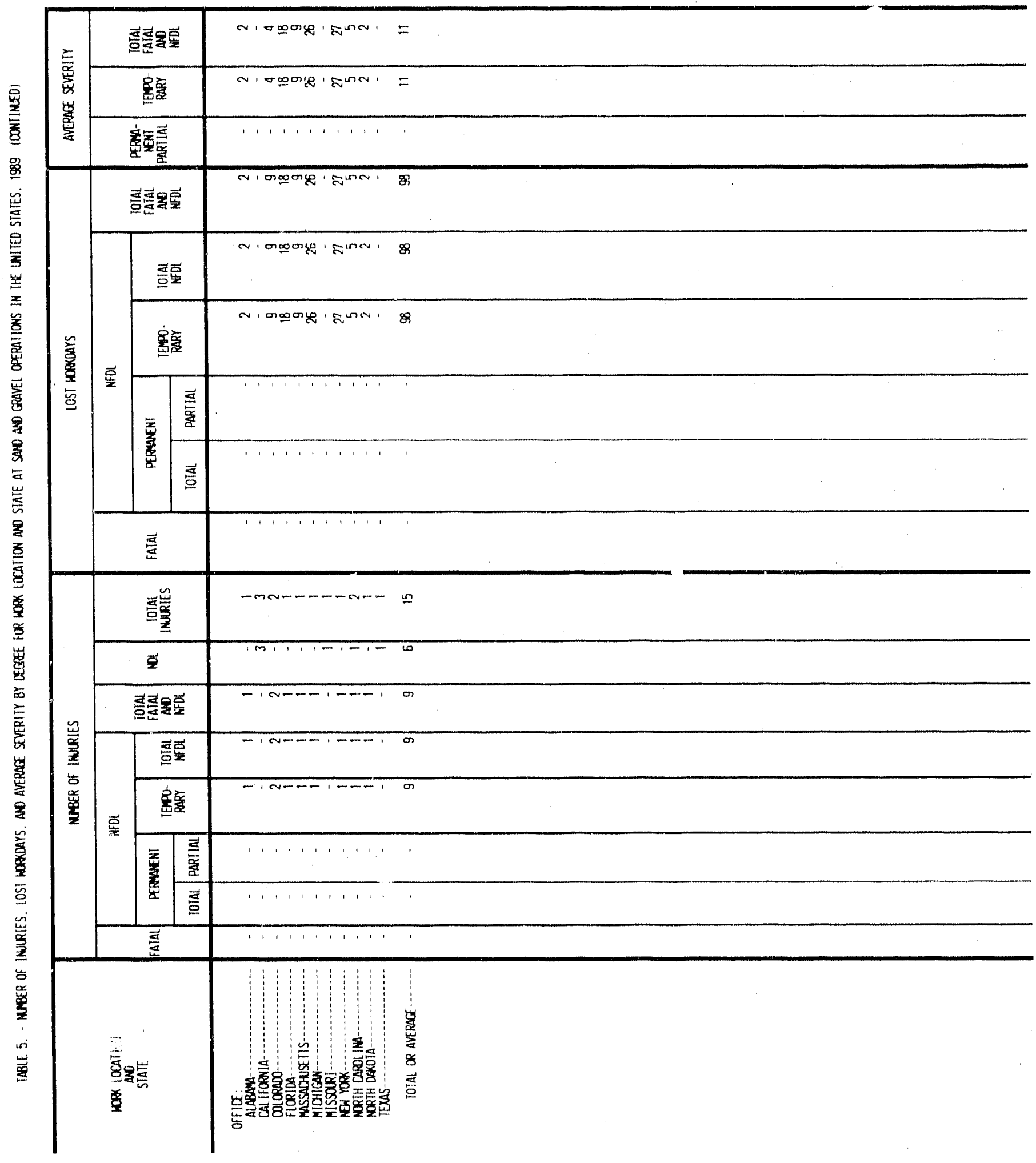




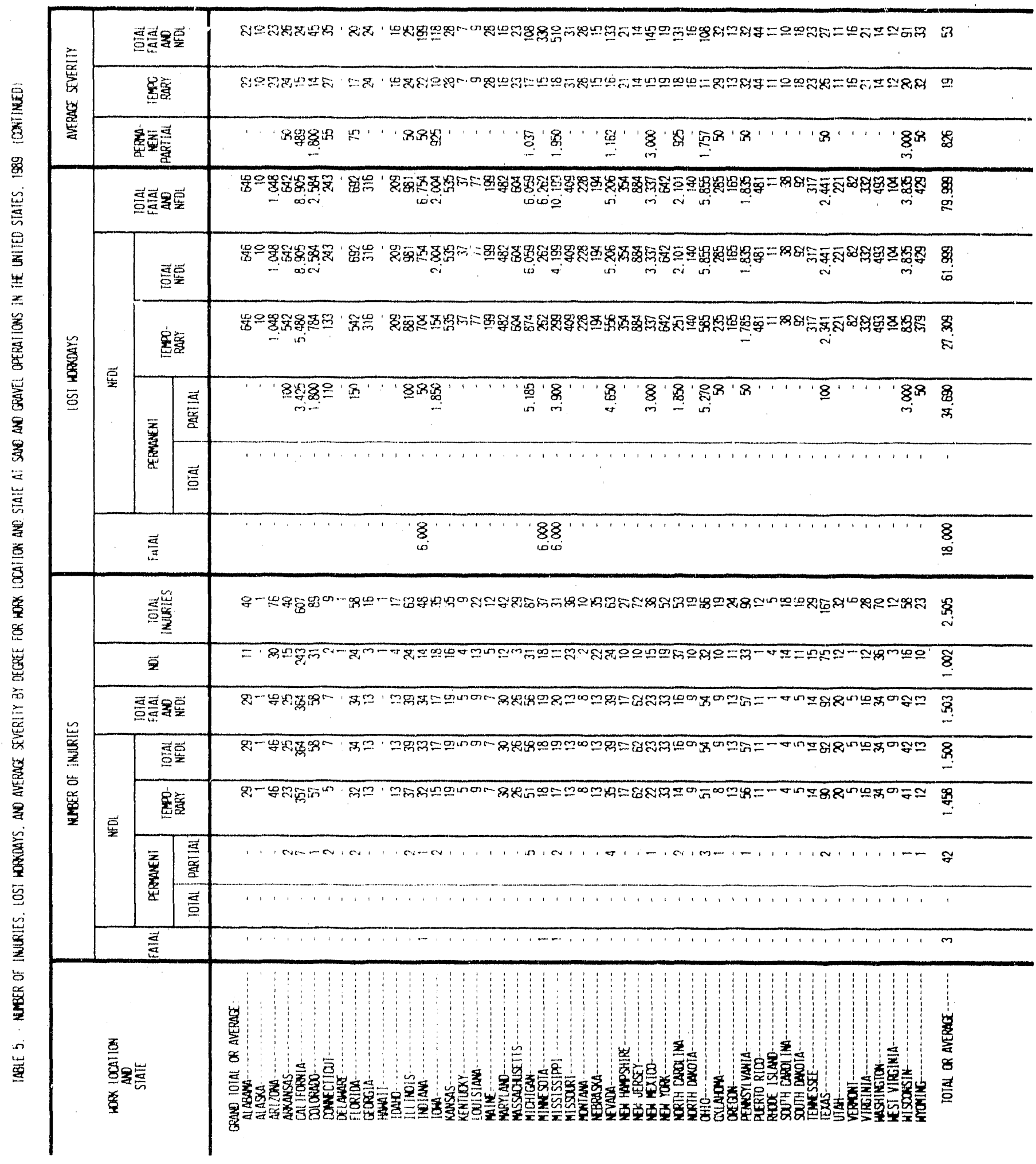




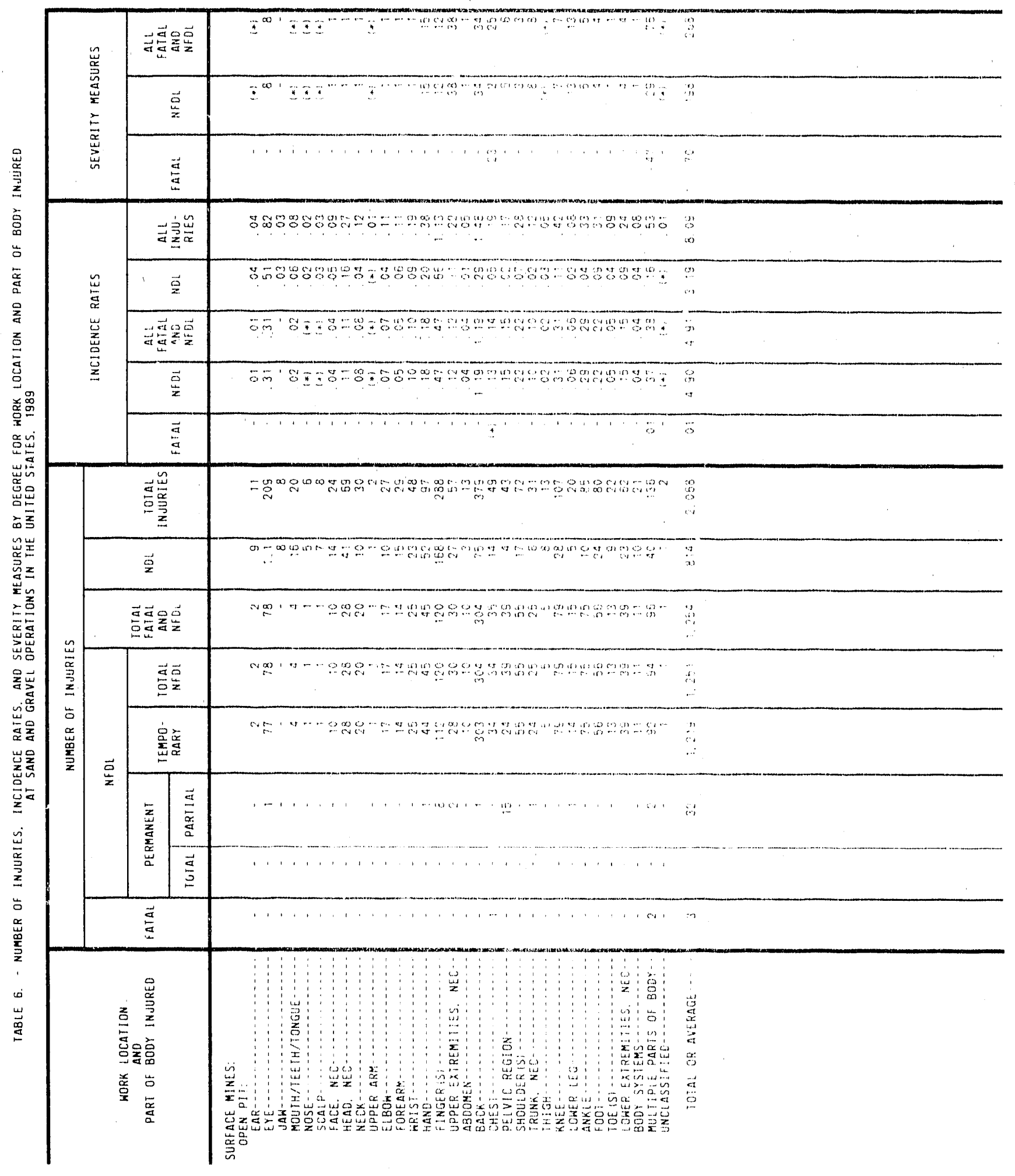




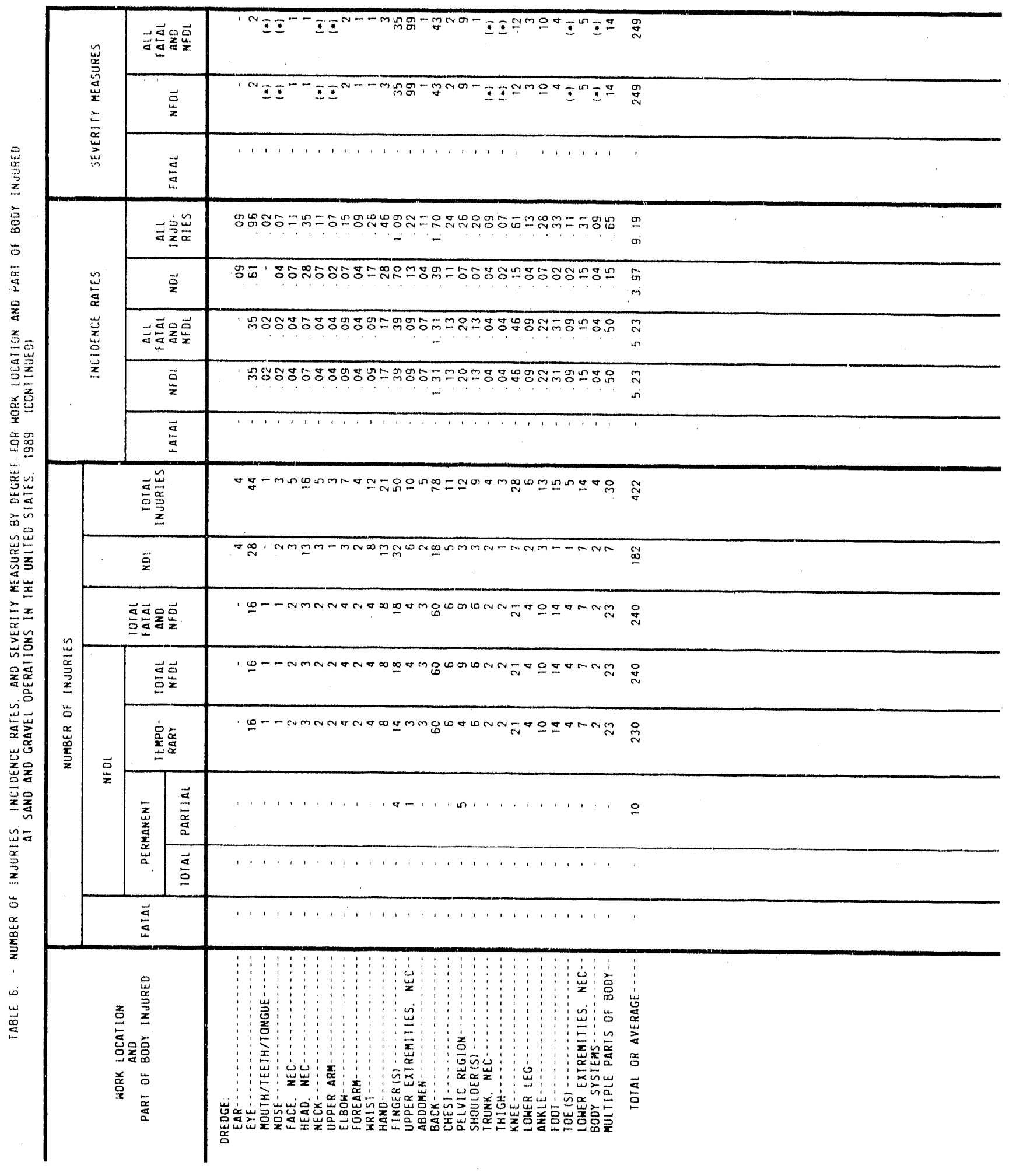




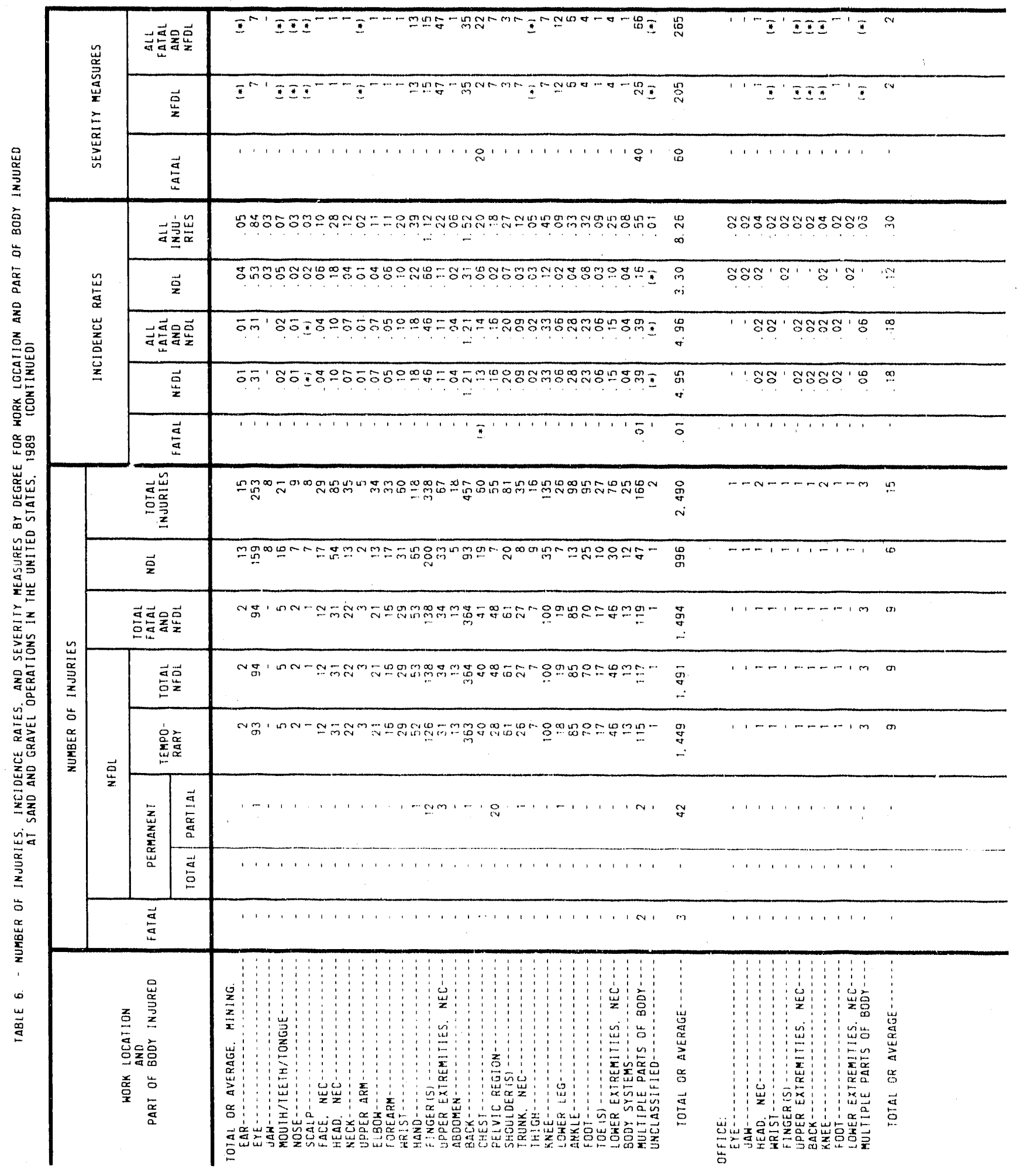




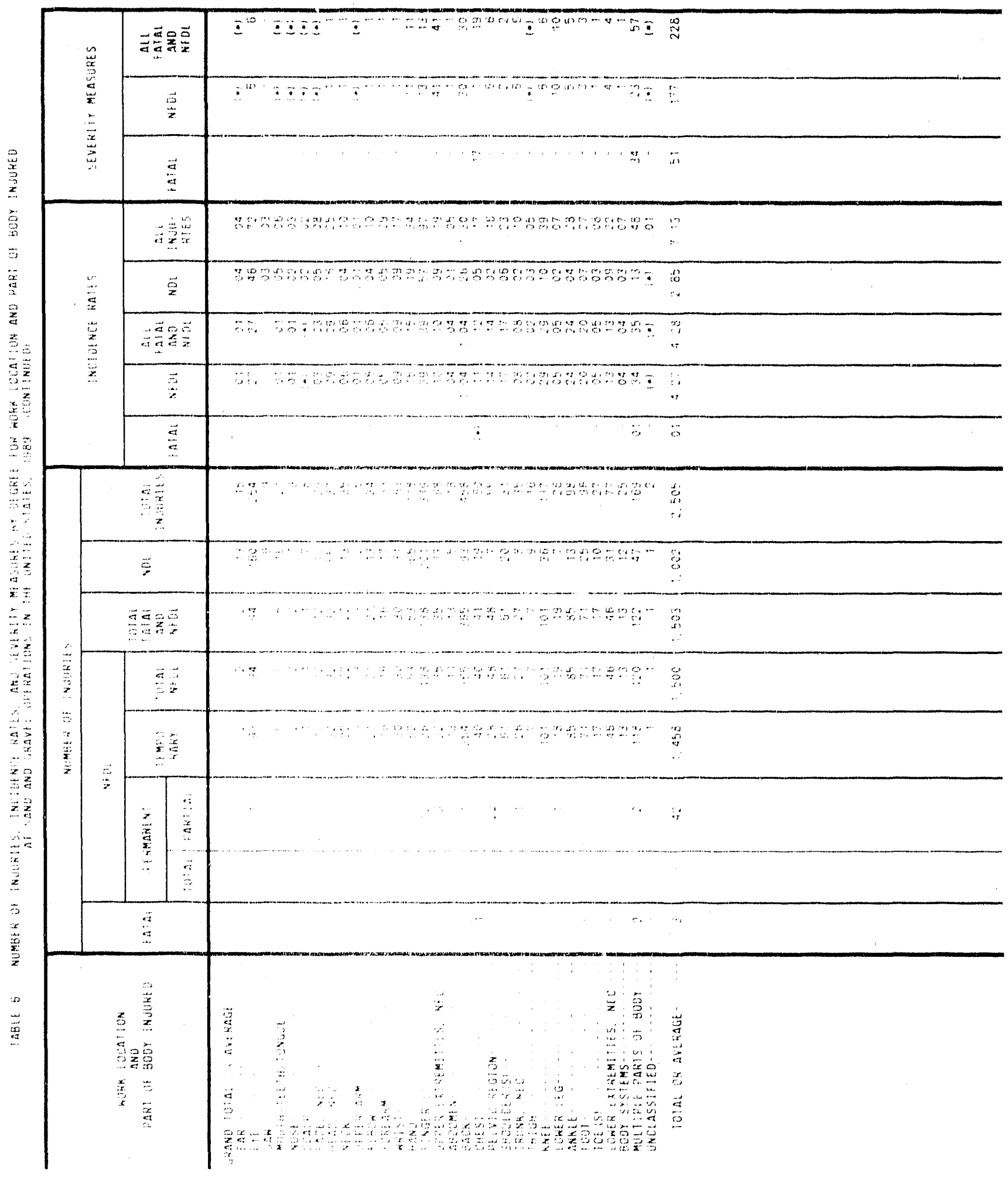




\begin{tabular}{|c|c|c|c|c|}
\hline \multirow{3}{*}{ 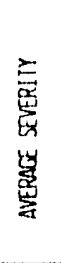 } & \multicolumn{2}{|c|}{ 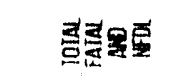 } & 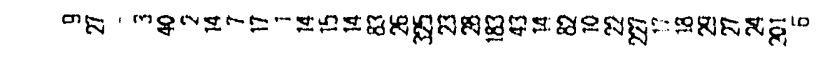 & if \\
\hline & \multicolumn{2}{|c|}{ 寡票 } & 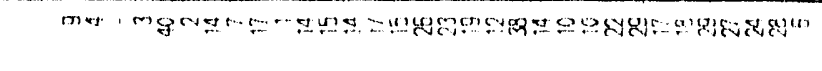 & 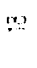 \\
\hline & \multicolumn{2}{|c|}{ 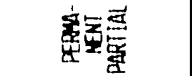 } & 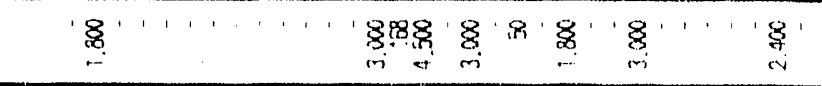 & 名 \\
\hline \multirow{6}{*}{ 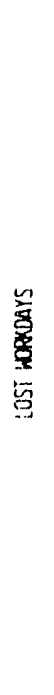 } & \multicolumn{2}{|c|}{ 흥춘욜 } & 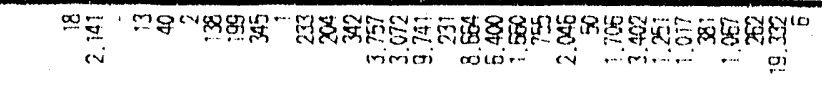 & 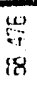 \\
\hline & \multicolumn{2}{|c|}{ 总要 } & 等 & \begin{tabular}{l}
0 \\
\hdashline \\
8
\end{tabular} \\
\hline & \multicolumn{2}{|c|}{ 害舜 } & 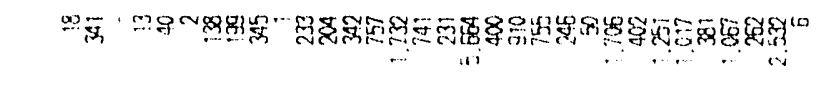 & 岁 \\
\hline & \multirow{2}{*}{ 豆 } & $\begin{array}{l}\text { 离 } \\
\text { 愛 }\end{array}$ & 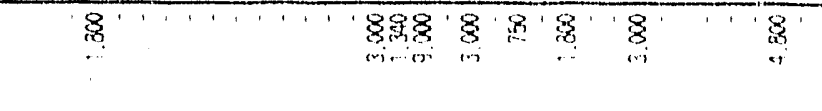 & 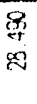 \\
\hline & & 菩 & '. & \\
\hline & \multicolumn{2}{|l|}{ 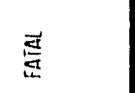 } & 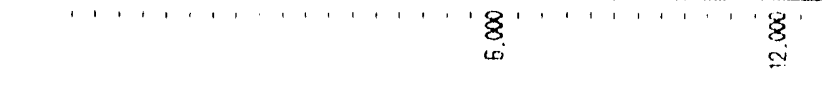 & 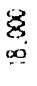 \\
\hline \multirow{8}{*}{ 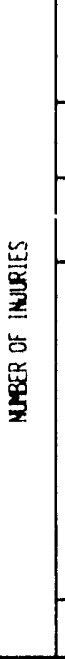 } & \multicolumn{2}{|c|}{ 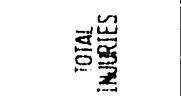 } & 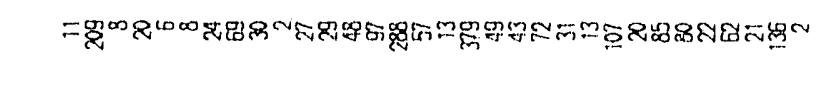 & 赵 \\
\hline & \multicolumn{2}{|l|}{$\bar{\Phi}$} & 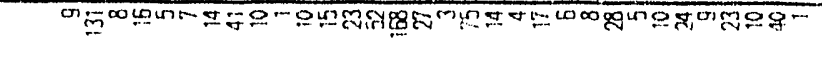 & $\stackrel{5}{: 0}$ \\
\hline & \multicolumn{2}{|c|}{ 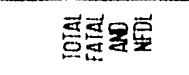 } & 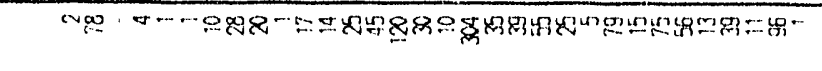 & Dִ \\
\hline & \multicolumn{2}{|c|}{ 흥물 } & 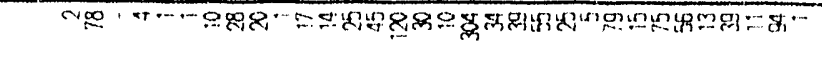 & 亝 \\
\hline & \multicolumn{2}{|c|}{ 鲁言 } & N & 界 \\
\hline & & 可 & $\cdots \infty \alpha_{1}, \ldots, m+\ldots, \ldots, \ldots, \ldots$ & $\Leftrightarrow$ \\
\hline & & $\underline{\underline{\Delta}}$ & $\cdots$ & \\
\hline & \multicolumn{2}{|l|}{$\frac{a t}{4}$} & $1,1,1$. & $(2)$ \\
\hline & \multicolumn{2}{|l|}{ 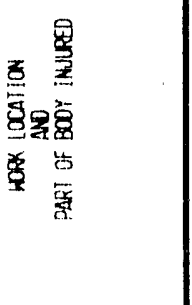 } & 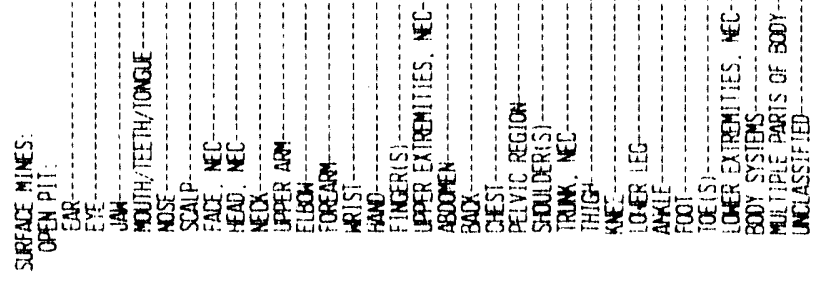 & 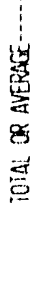 \\
\hline
\end{tabular}




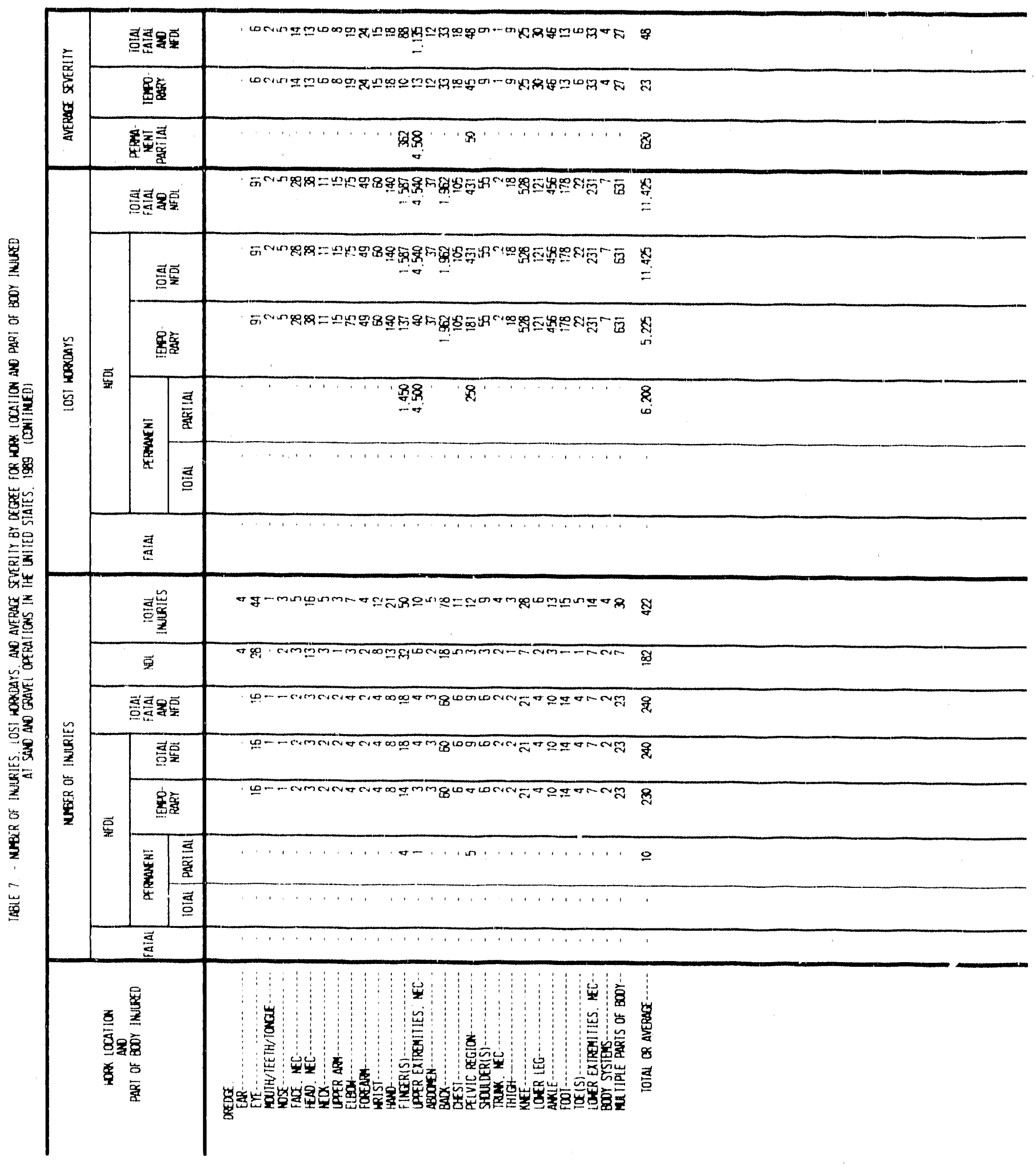




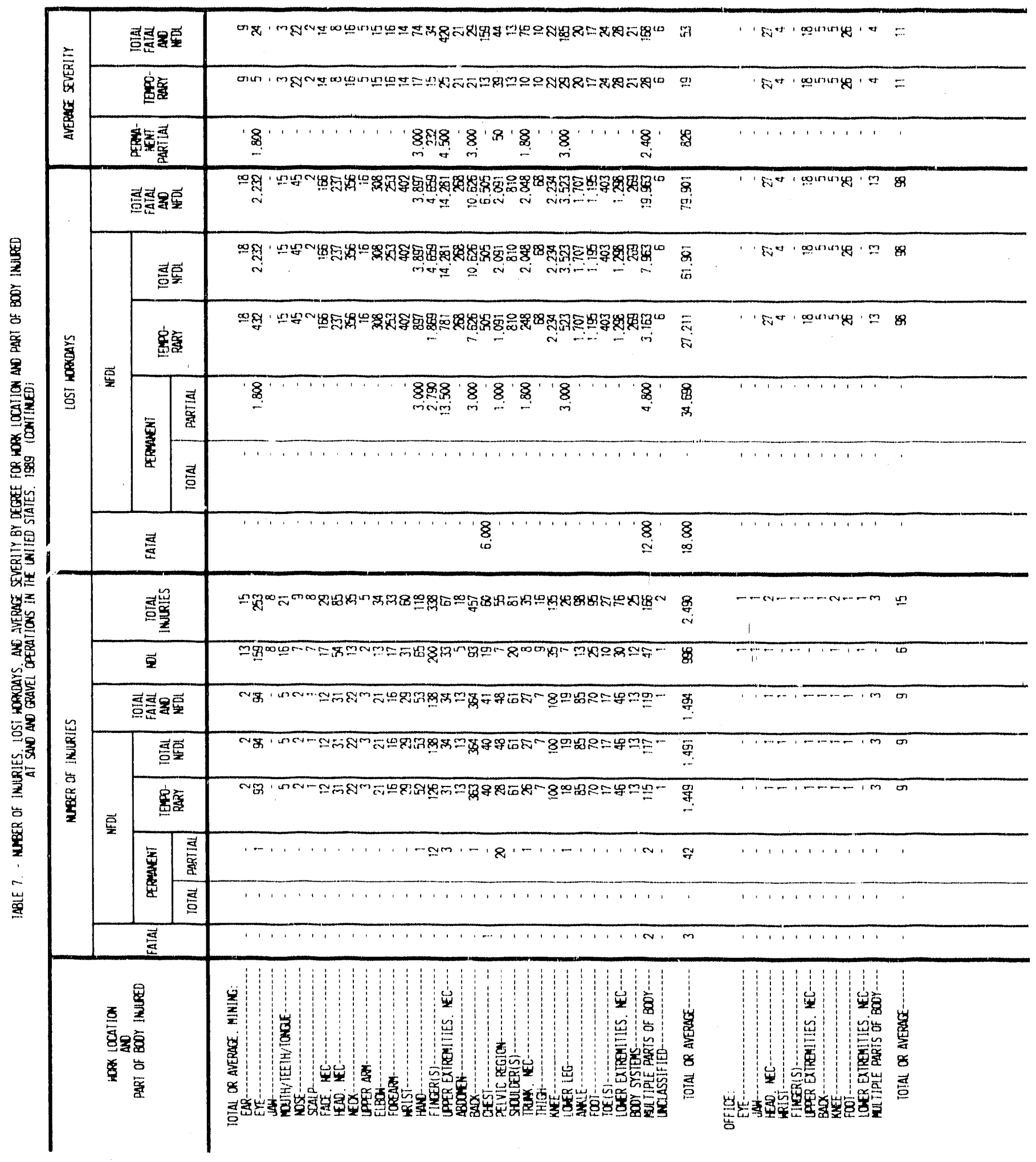




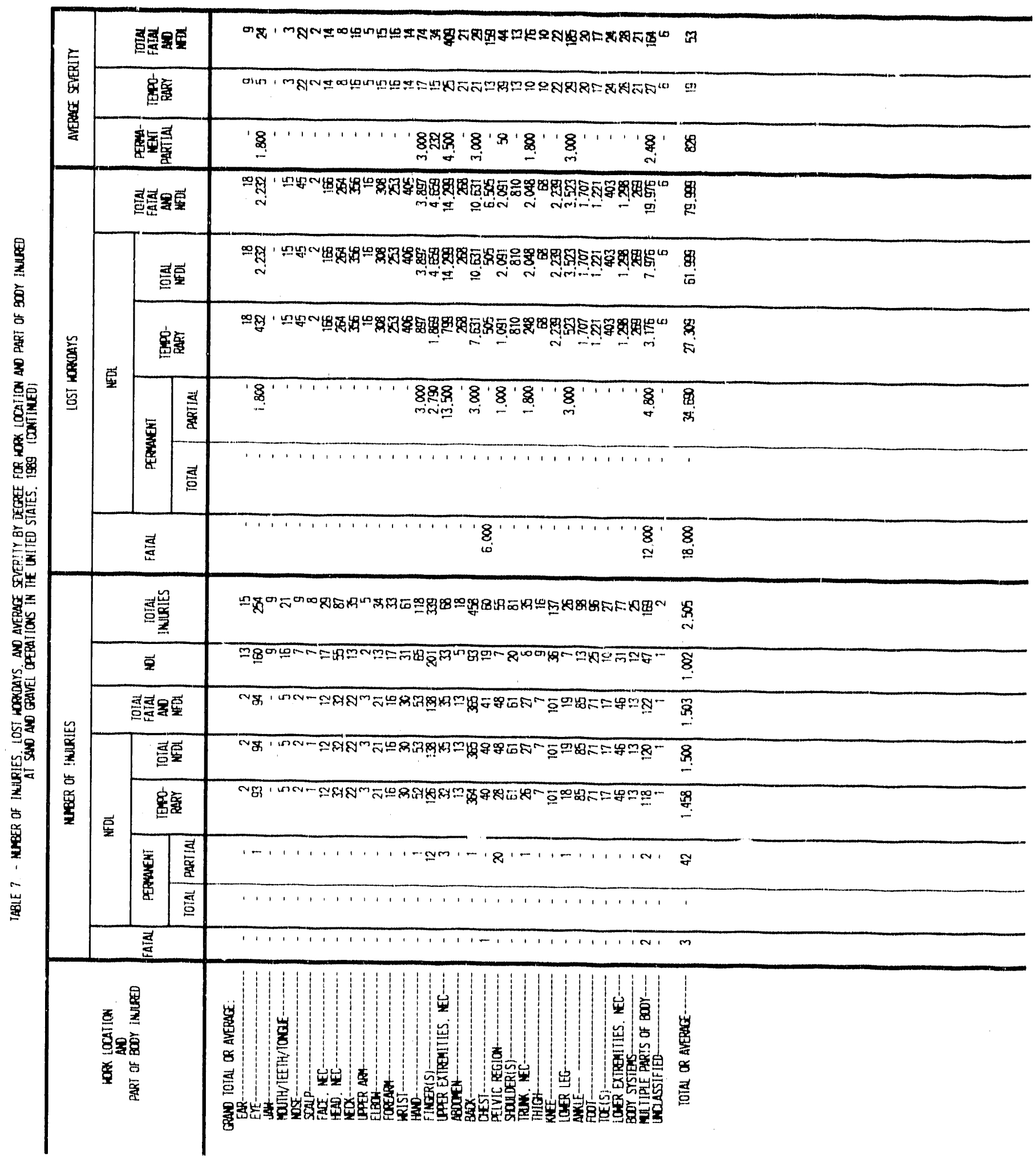




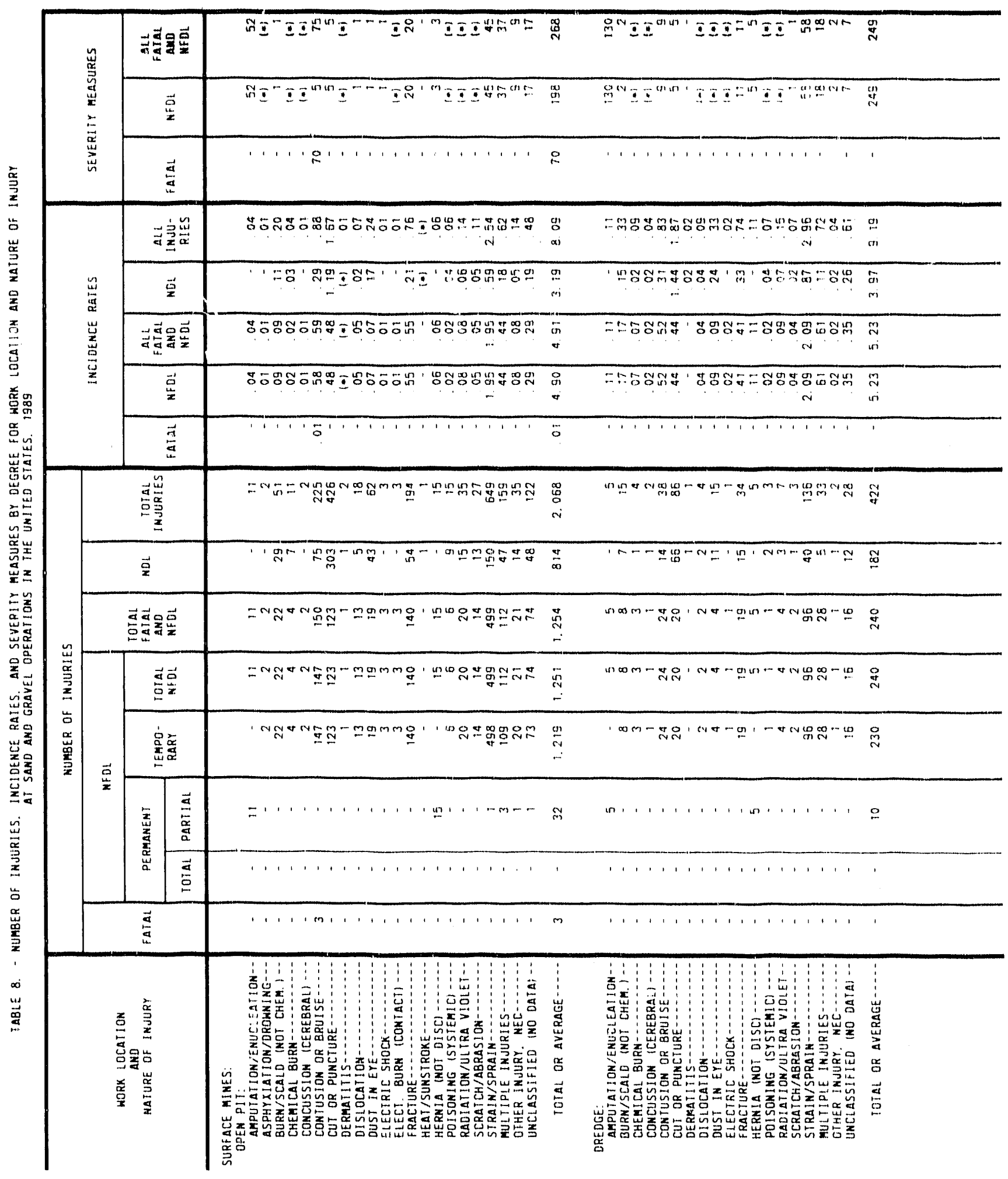




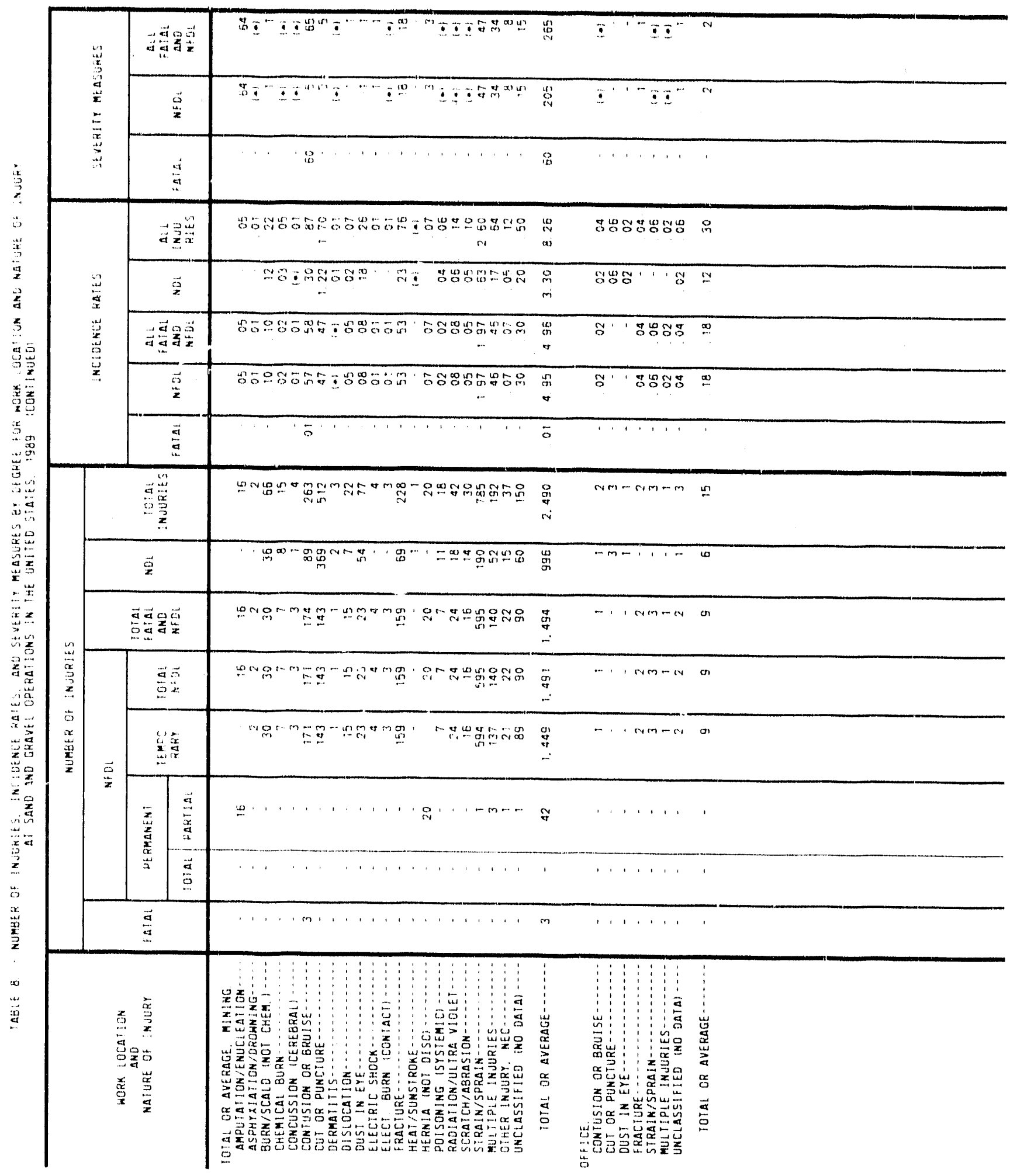




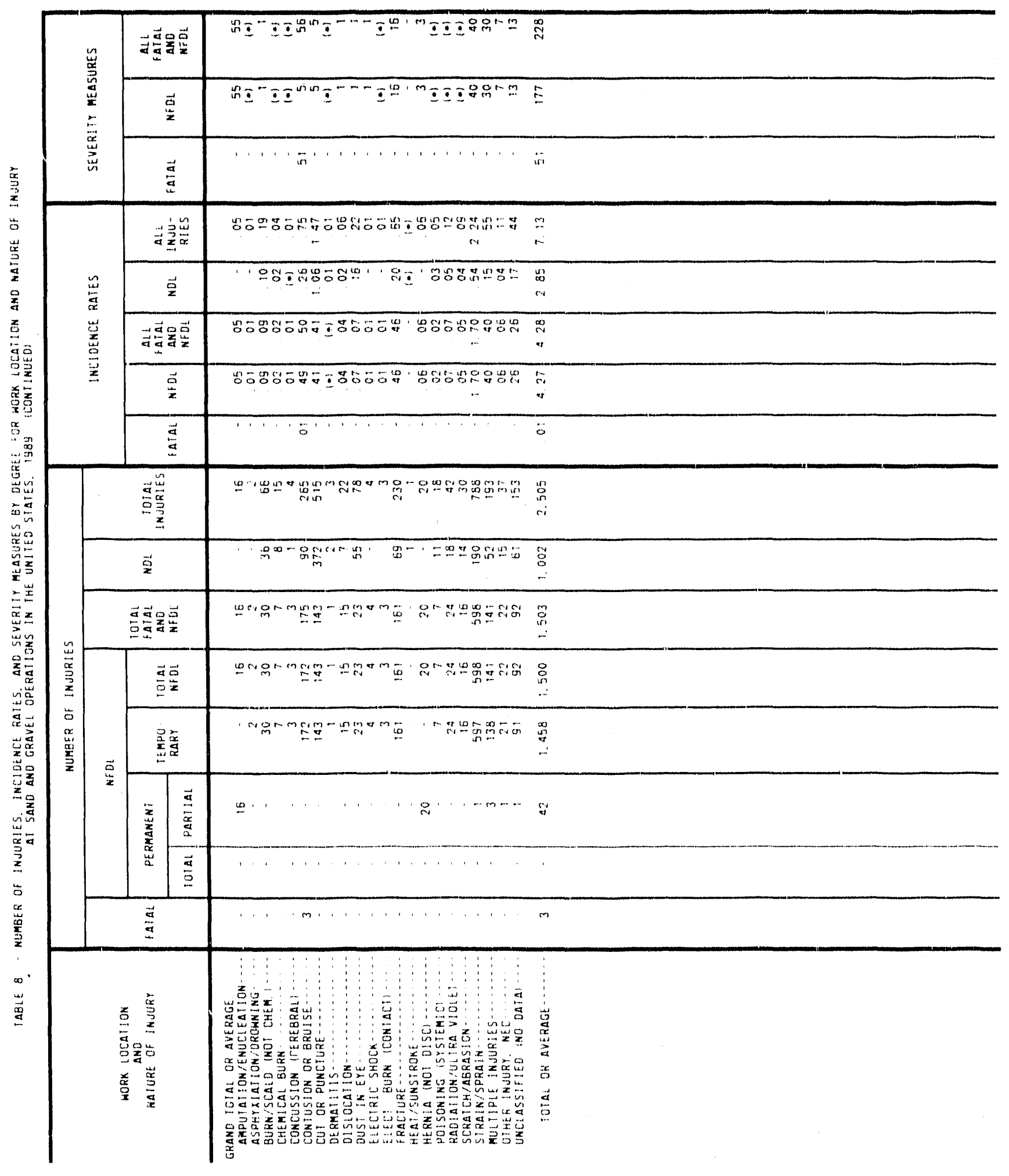




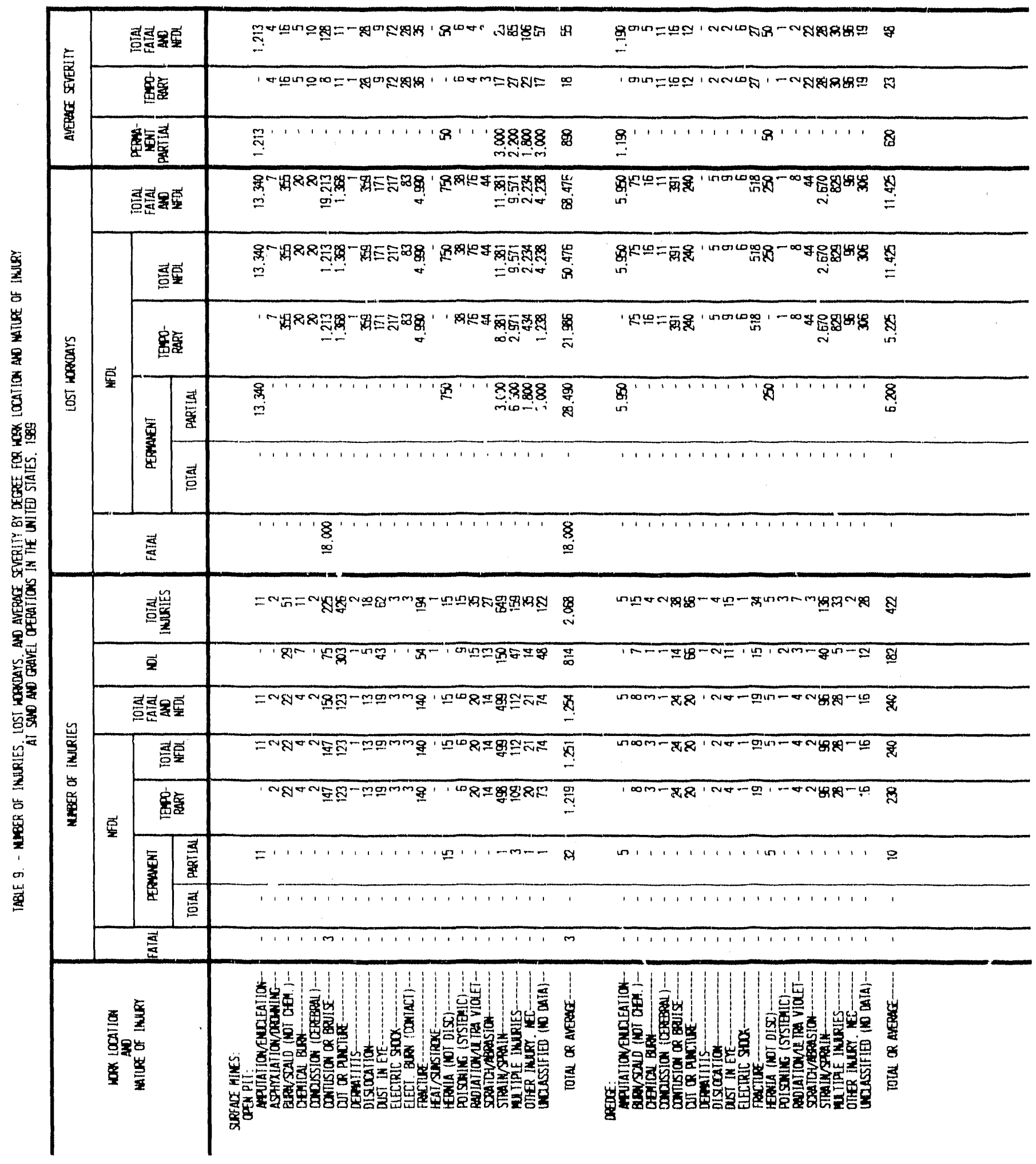




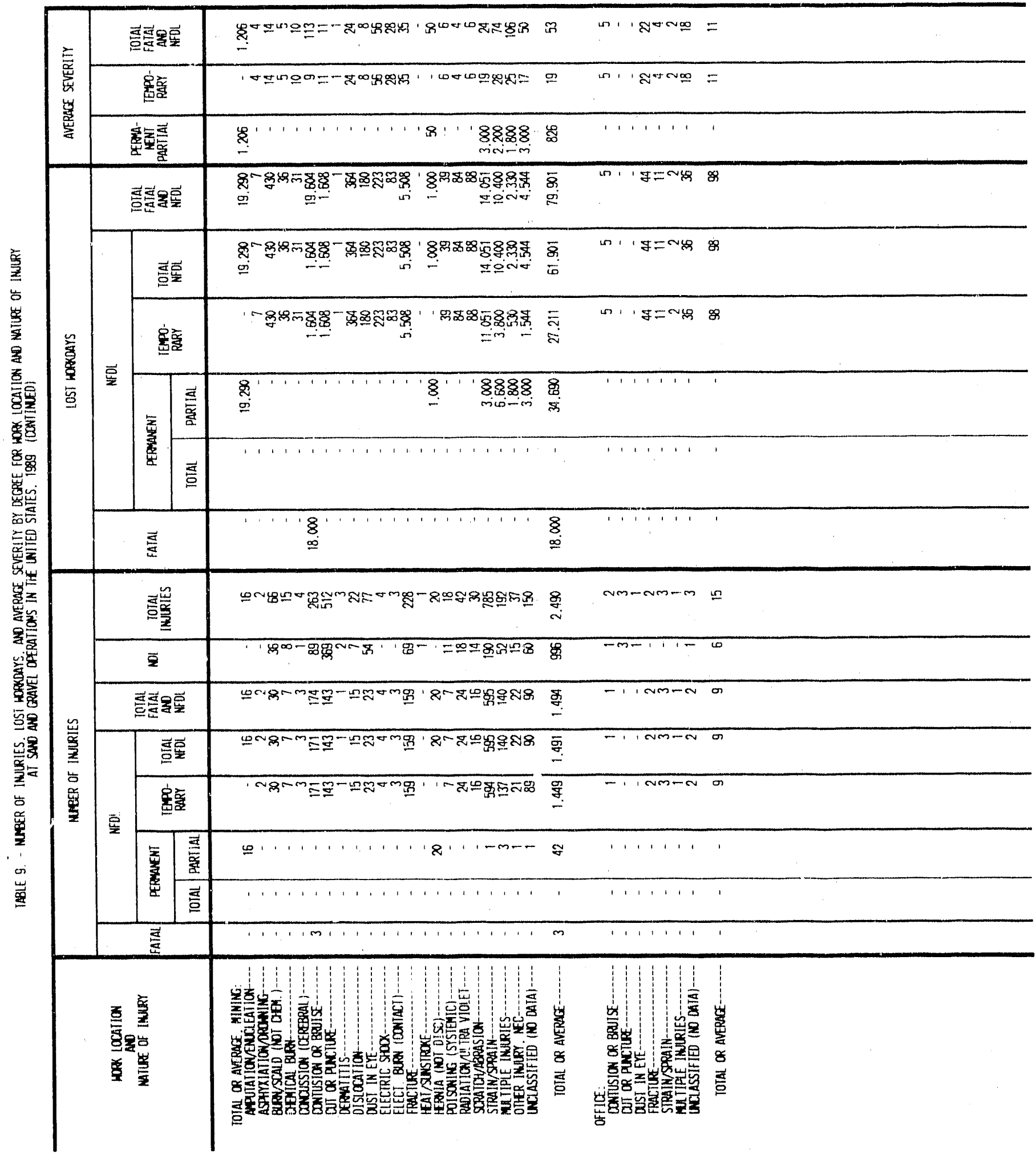




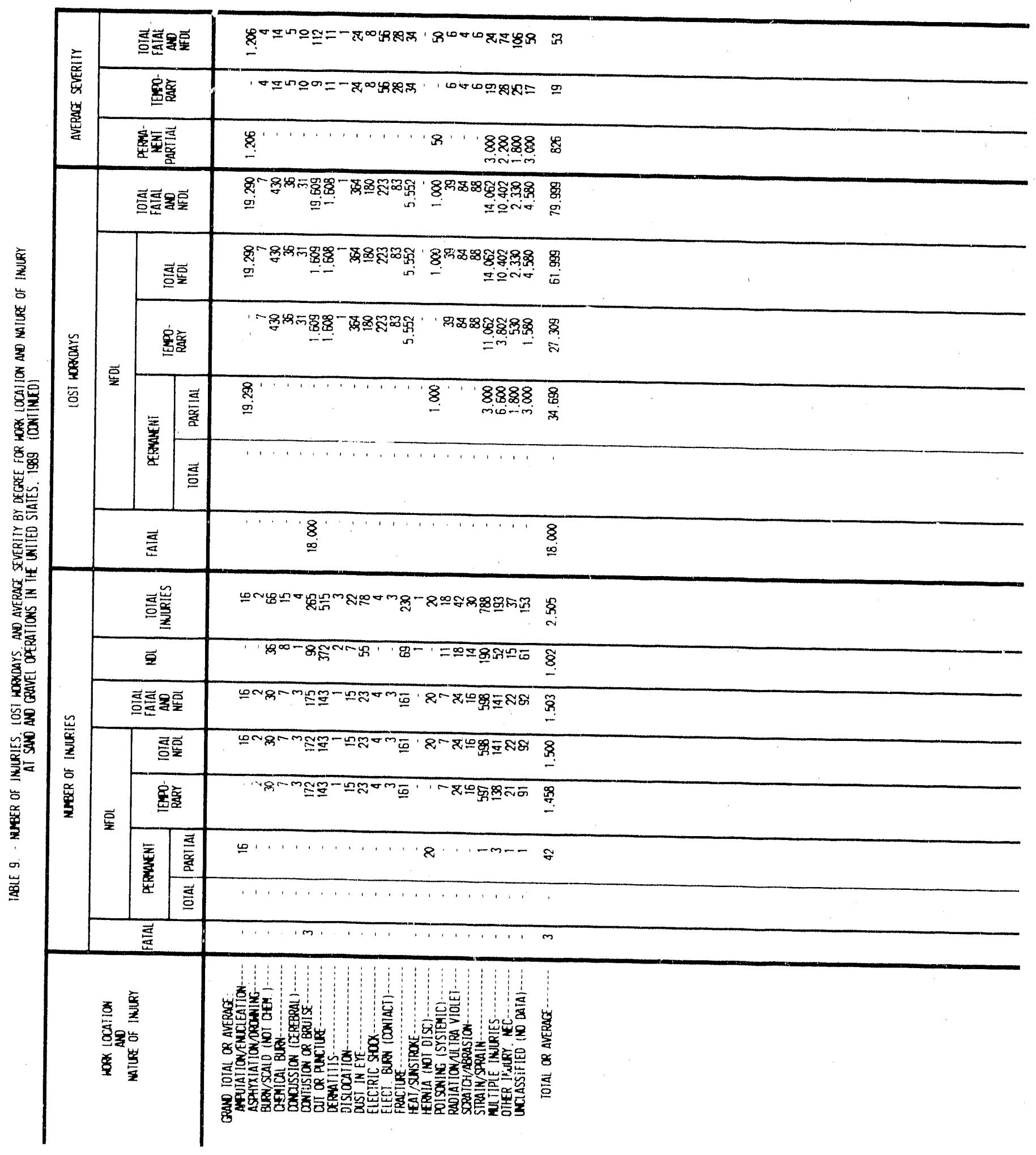




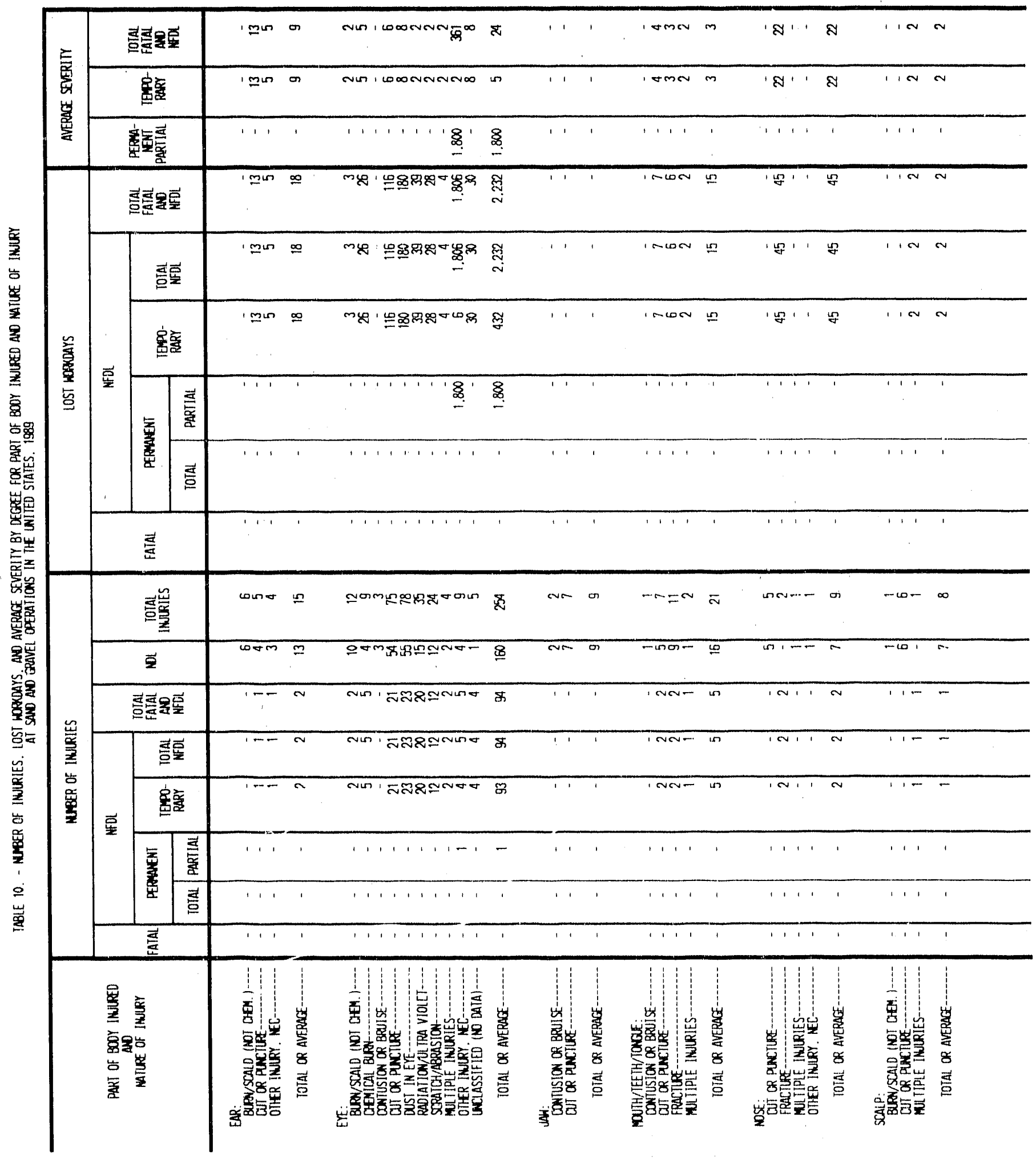




\begin{tabular}{|c|c|c|c|c|c|c|c|c|c|c|c|c|}
\hline \multirow{3}{*}{ 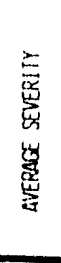 } & \multicolumn{2}{|c|}{ 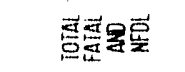 } & \& $\cdots$ & \pm & goveng , ' Fun & $\infty$ & compgog & 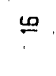 & $\pm 1-\infty$ & un & 으매테뮤 & 15 \\
\hline & \multicolumn{2}{|c|}{ 悹愛 } & $\mathbb{N}+m+\infty$ & \pm & goveng ' ' =mr: & $\infty$ & usmmg & 느 & $\pm \cdots$ & in & 으태트메 & 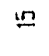 \\
\hline & \multicolumn{2}{|c|}{ 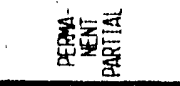 } & 1,1 & ' & $1,1,1,1,1$ & ' & $1+1$ & , & 1,1 & ' & $1,1,1$ & . \\
\hline \multirow{6}{*}{ 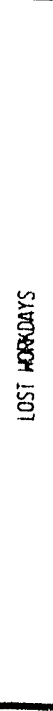 } & \multicolumn{2}{|c|}{ 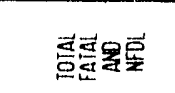 } & 며의 & 98 & 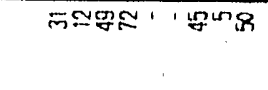 & 苾 & gnलs & 量 & $\pm 1-\cdots$ & 트 & 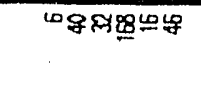 & 学 \\
\hline & \multirow{4}{*}{ 垔 } & 宕㑒 & 욤요 & 18 & mহgू̃ ' ' & 苾 & gimg & 量 & $\pm 1 \cdot-$ & $\infty$ & 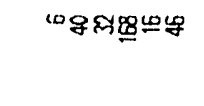 & $\mathscr{\&}$ \\
\hline & & 题舜 & $\underline{\Xi} \because 9$ & 9 & 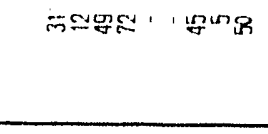 & 更 & onmg桀 & 蛊 & $\pm 1-\infty$ & $\underline{\underline{0}}$ & 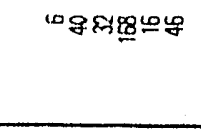 & $\stackrel{\infty}{\infty}$ \\
\hline & & 営 & ' ' ' ' & ' & 111,1111 & ' & 1,1 & ' & 111 & ' & 111,11 & 1 \\
\hline & & 总 & $\therefore \cdot 1$ & ' & $1,1,1,1,1$ & ' & 1,1 & . & 1,1 & ' & $1,1,1$ & ' \\
\hline & \multicolumn{2}{|l|}{ 远 } & $1 \cdot 1 \cdot 1$ & 1 & $1,1,1,1$, & 1 & 1,1 & ' & 1,1 & i & 1 & 1 \\
\hline \multirow{8}{*}{ 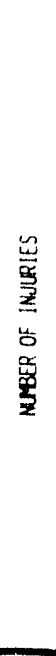 } & \multicolumn{2}{|c|}{ 点贺骂 } & movston & $g$ & 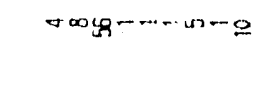 & 5 & mmyen & म्ल & $m-n$ & w & rustm-lo & ক \\
\hline & \multicolumn{2}{|l|}{$\ddot{2}$} & $100-\theta$ & $=$ & $-m \div 1--1 m$ & 出 & $n=1$ & $m$ & 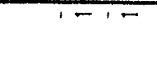 & 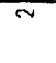 & ग-m, & $m$ \\
\hline & \multicolumn{2}{|c|}{ 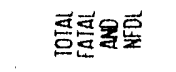 } & $m \cdot a \cdot m$ & $\simeq$ & ming : & ह্ & $m+n m$ & \& & -+-- & $m$ & $m--0-\infty$ & $\bar{v}$ \\
\hline & \multicolumn{2}{|c|}{ 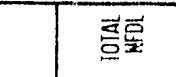 } & m. nin & $s$ & munno-1. & ஜ & $m-w m$ & ริ & $-1-7$ & $m$ & ma-o-c & $\overline{2}$ \\
\hline & \multicolumn{2}{|c|}{ 题高 } & in. $91 \mathrm{in}$ & $\simeq$ & minns-i, $0-m$ & ह & $m-w m$ & $\bar{N}$ & $-1--$ & $m$ & moro- & $\bar{\pi}$ \\
\hline & \multirow{2}{*}{ 勇 } & 高 & $1,1,1$ & ' & $1,1,1,1$ & ' & $1+1$ & ' & 1,1 & ' & $1,1,1,1$ & 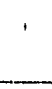 \\
\hline & & 평 & $1,1,1$ & ' & $1,1,1,1$ & ' & 111 & ' & 111 & ' & $1,1,11$ & '. \\
\hline & \multicolumn{2}{|l|}{$\frac{\mathrm{z}}{2}$} & $1,1,1$ & ' & ' ' ' ' & & 1,1 & ' & ' & 1 & 111 & ' \\
\hline & 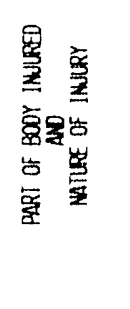 & & 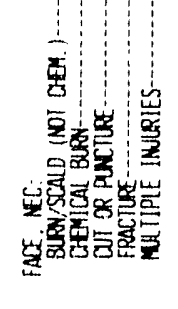 & 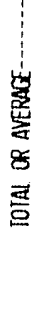 & 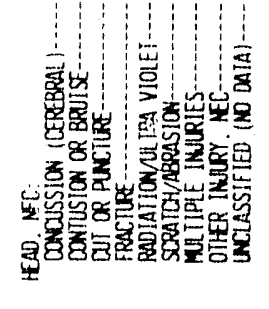 & 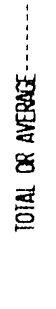 & 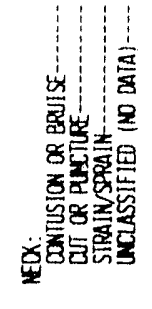 & 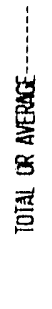 & 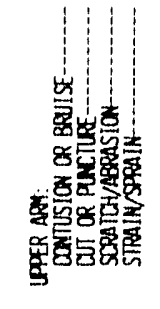 & 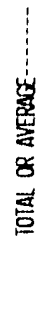 & 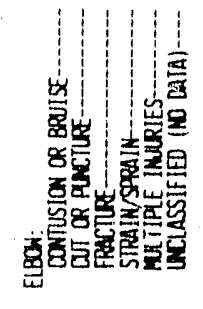 & 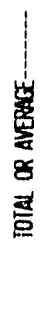 \\
\hline
\end{tabular}




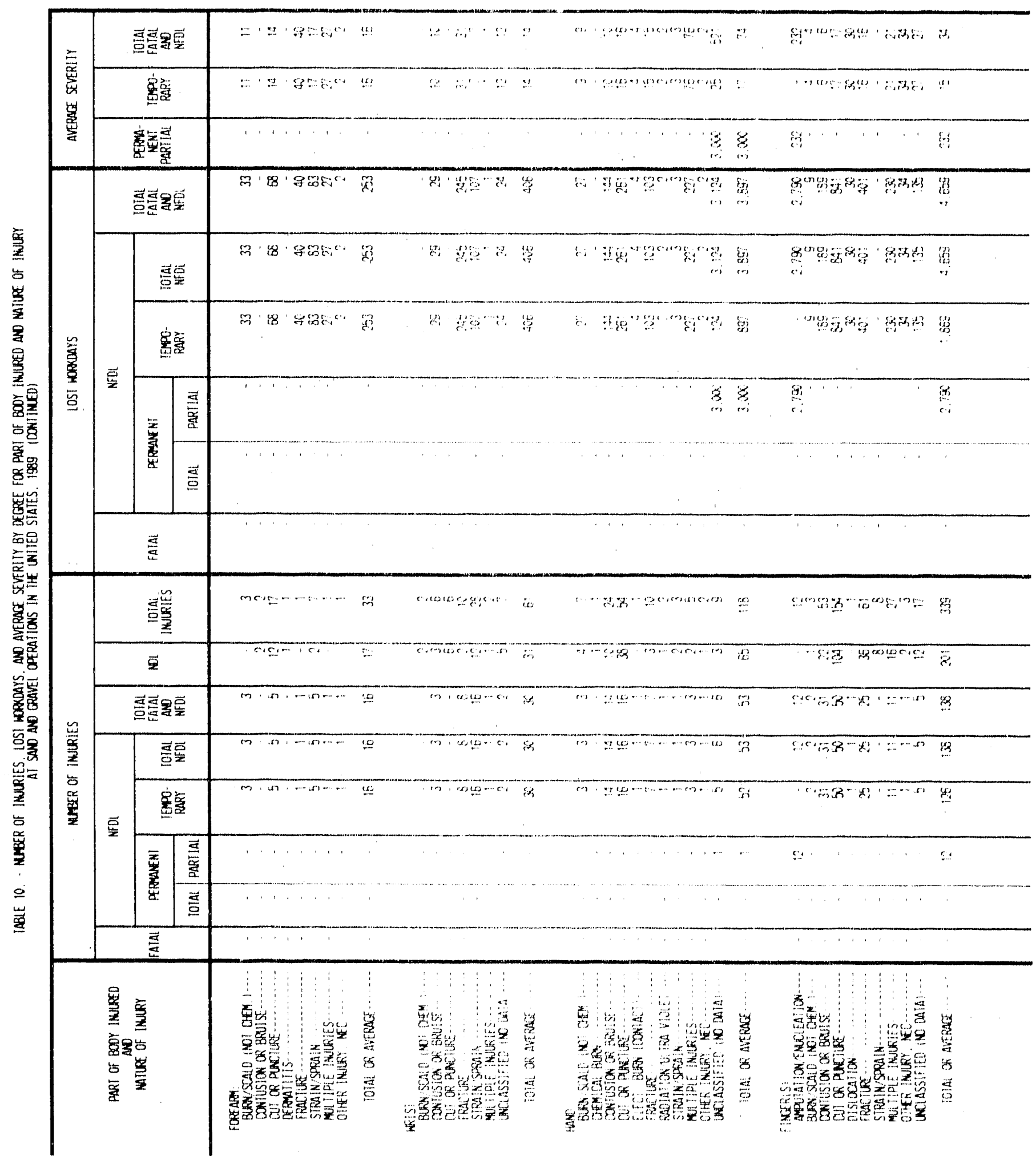




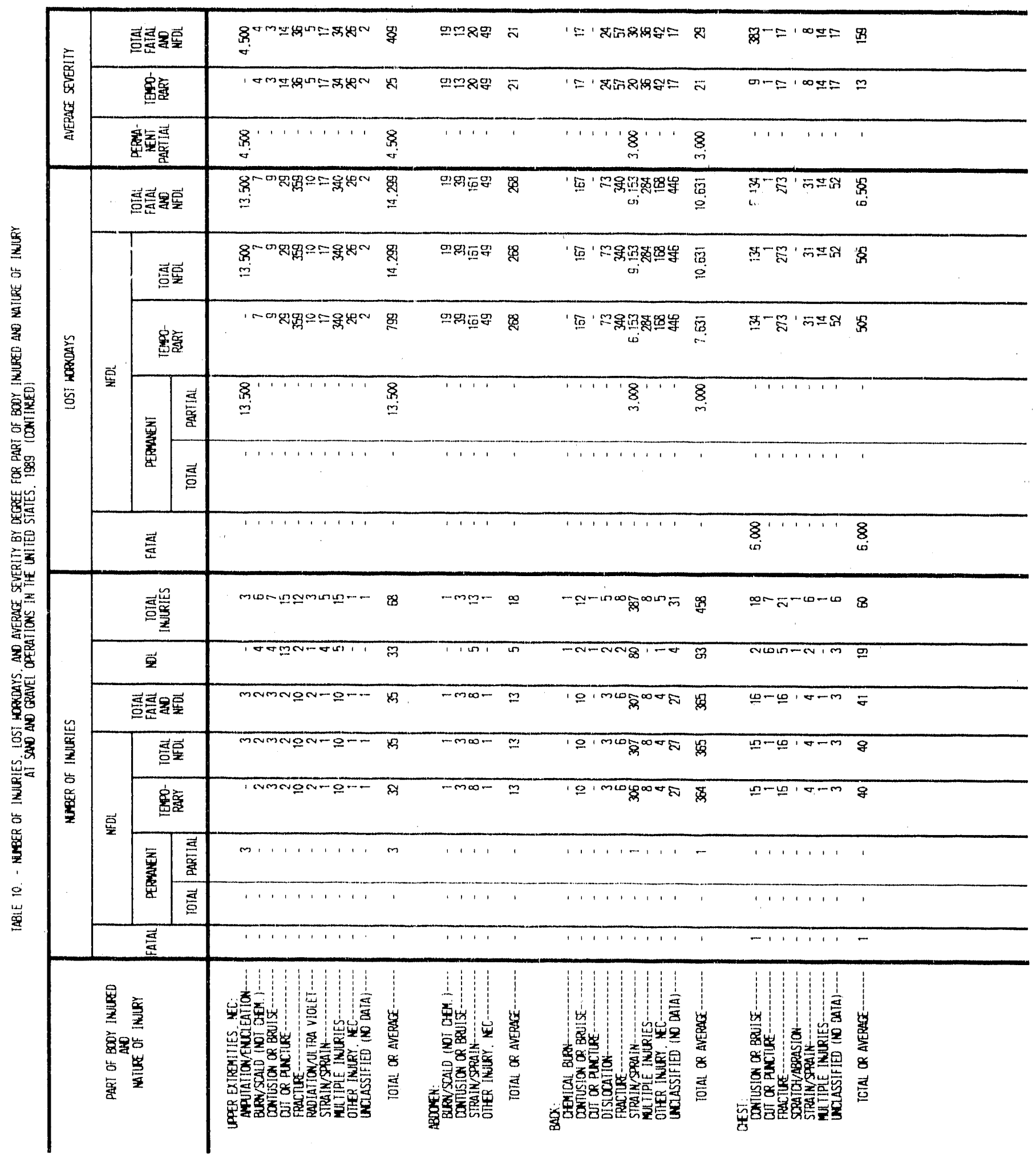




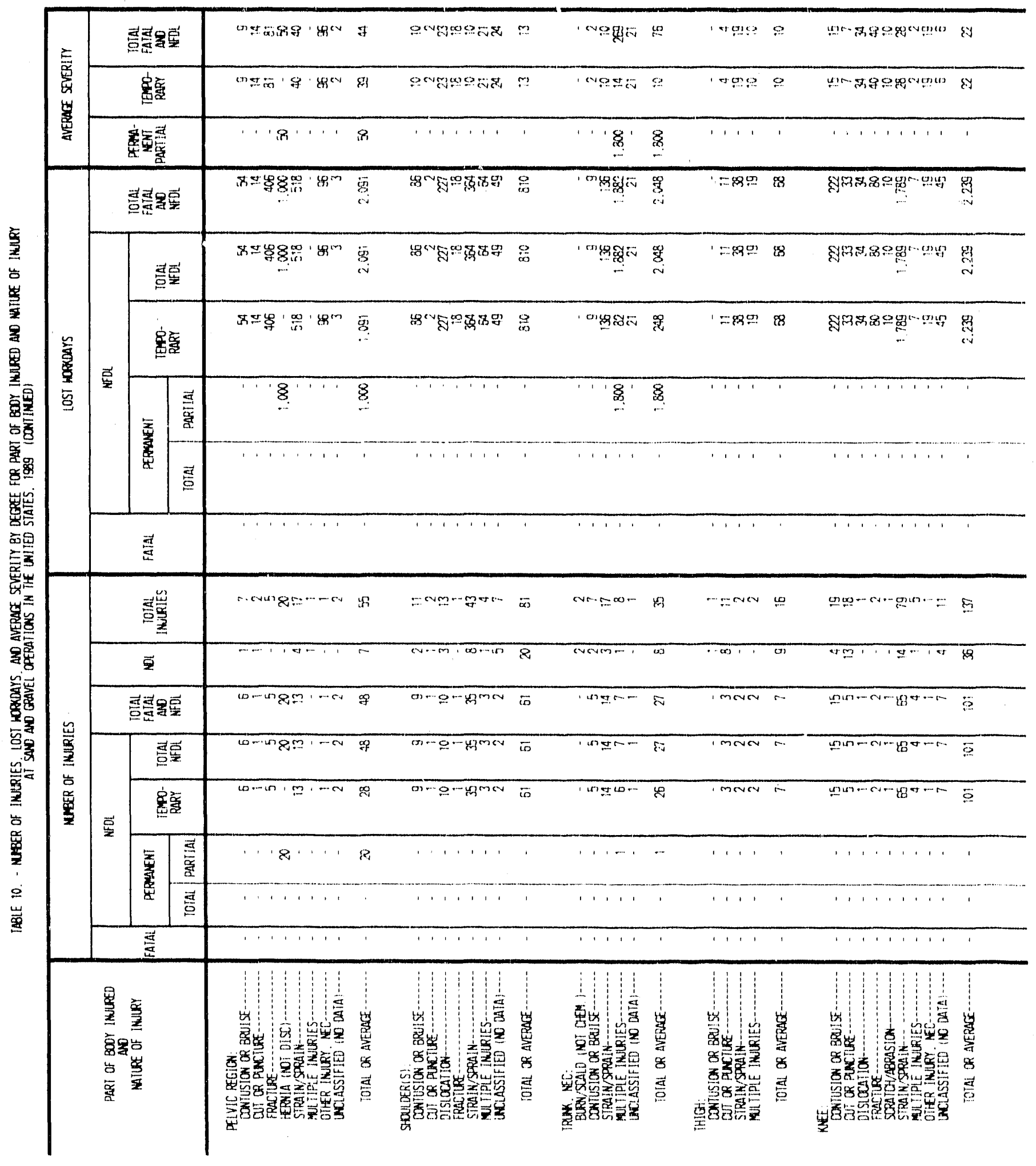




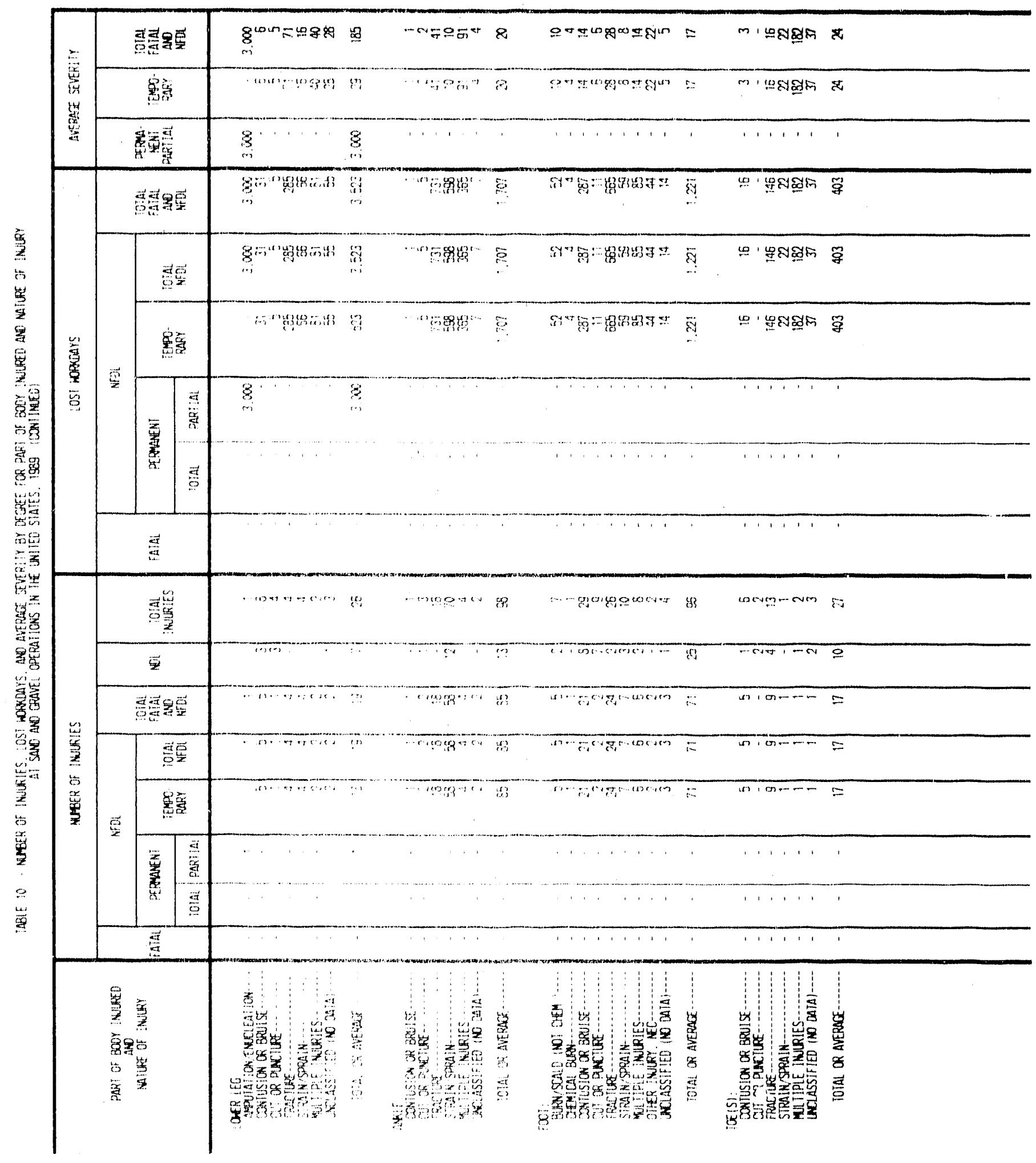




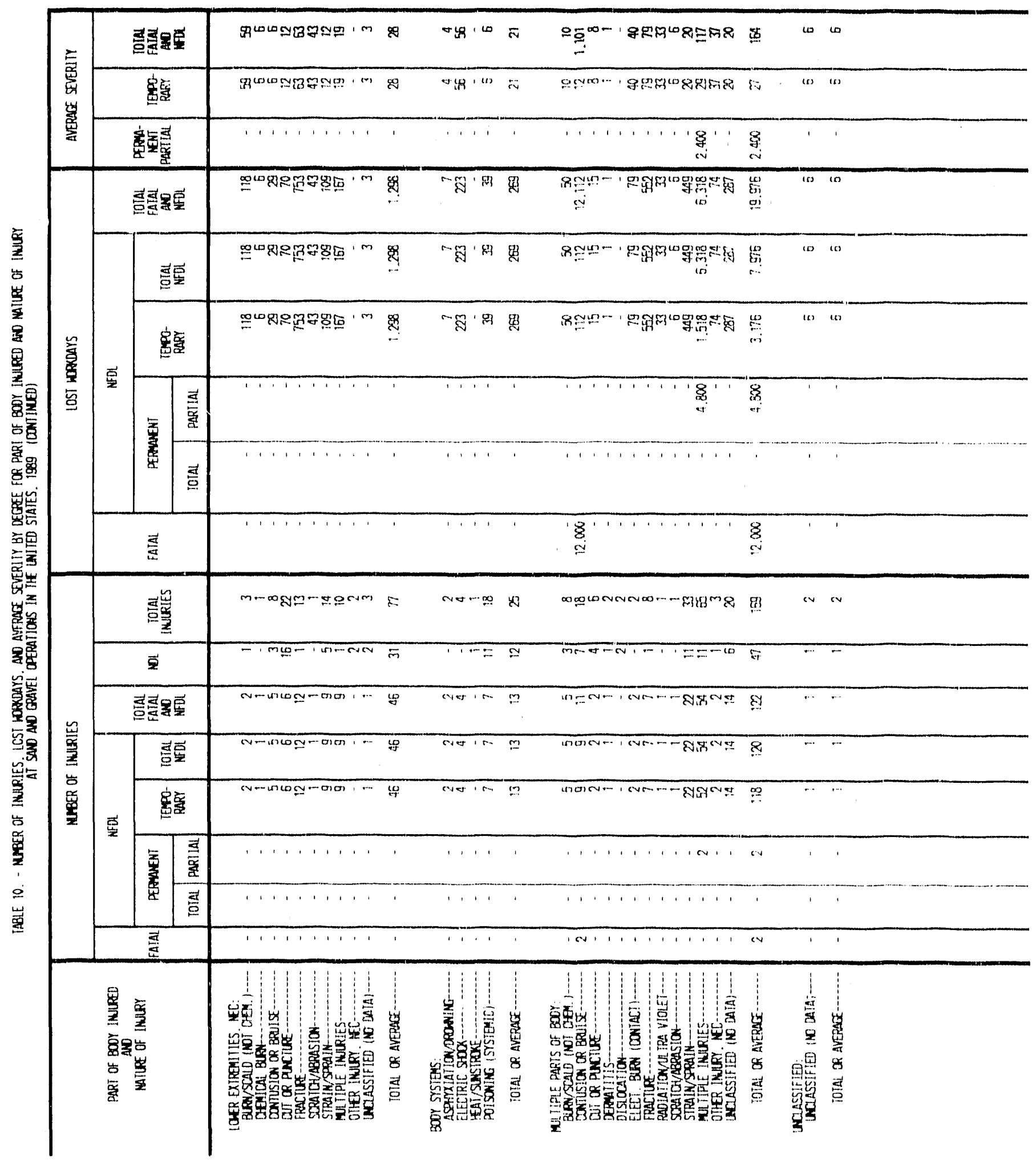




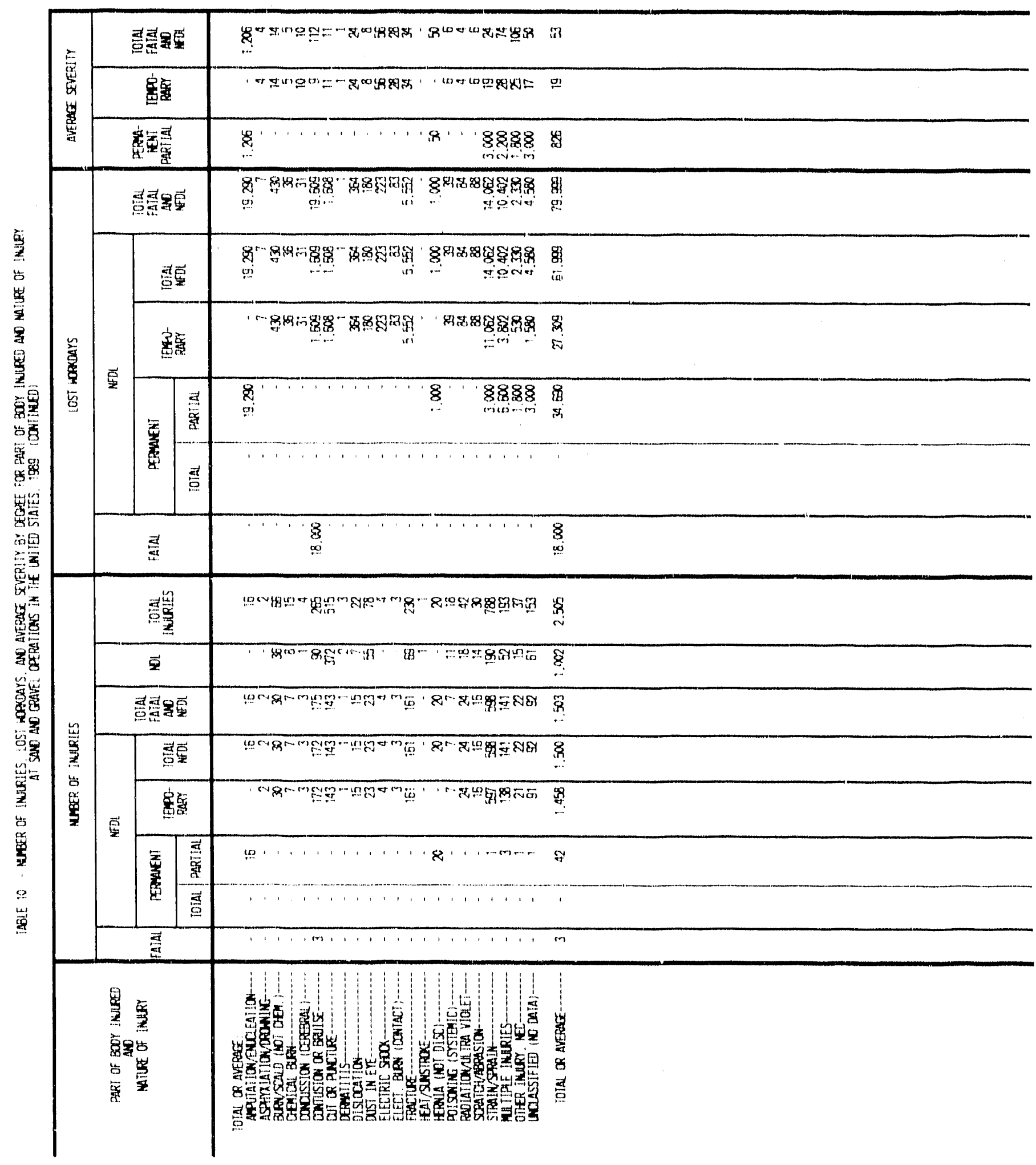




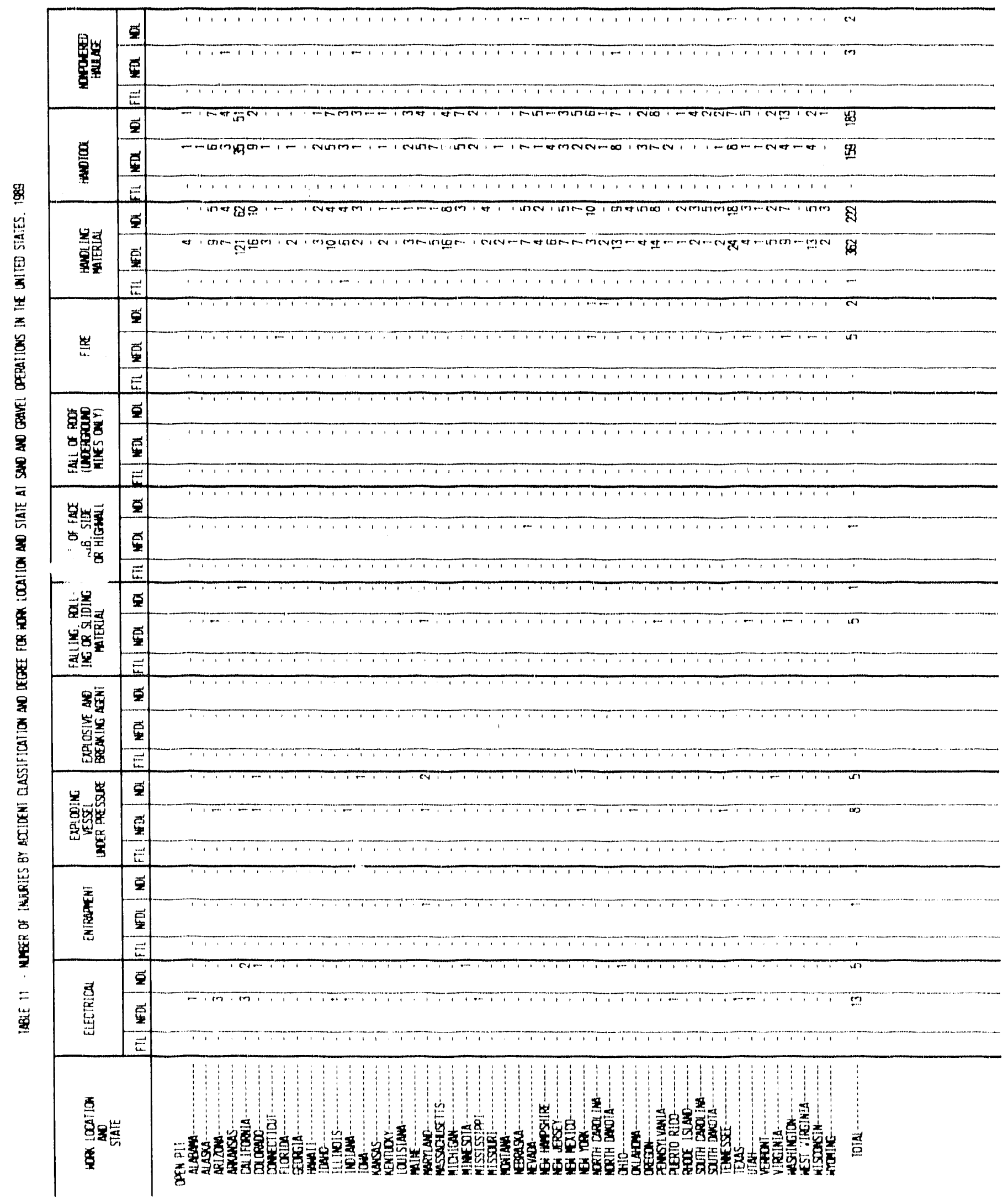




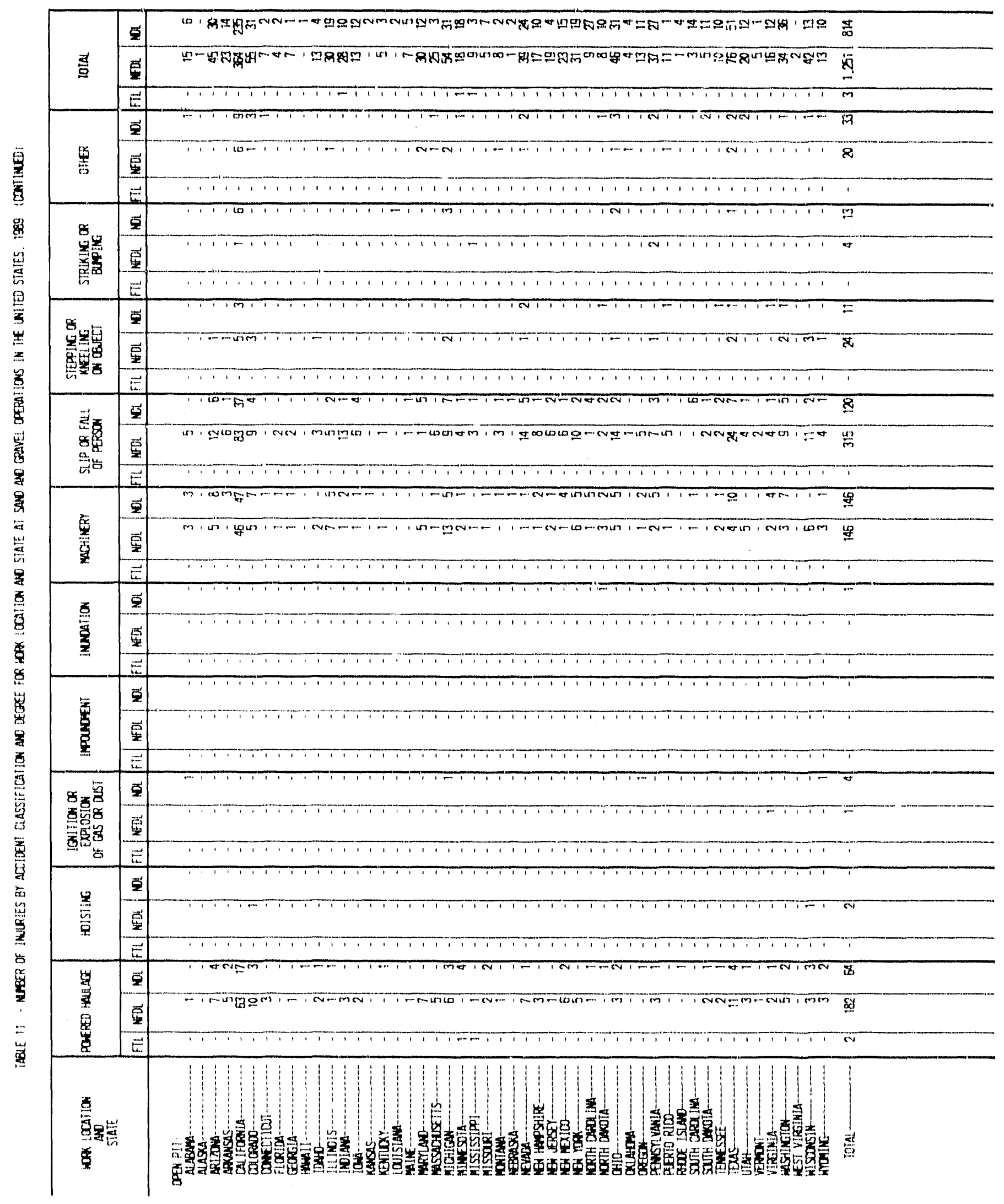




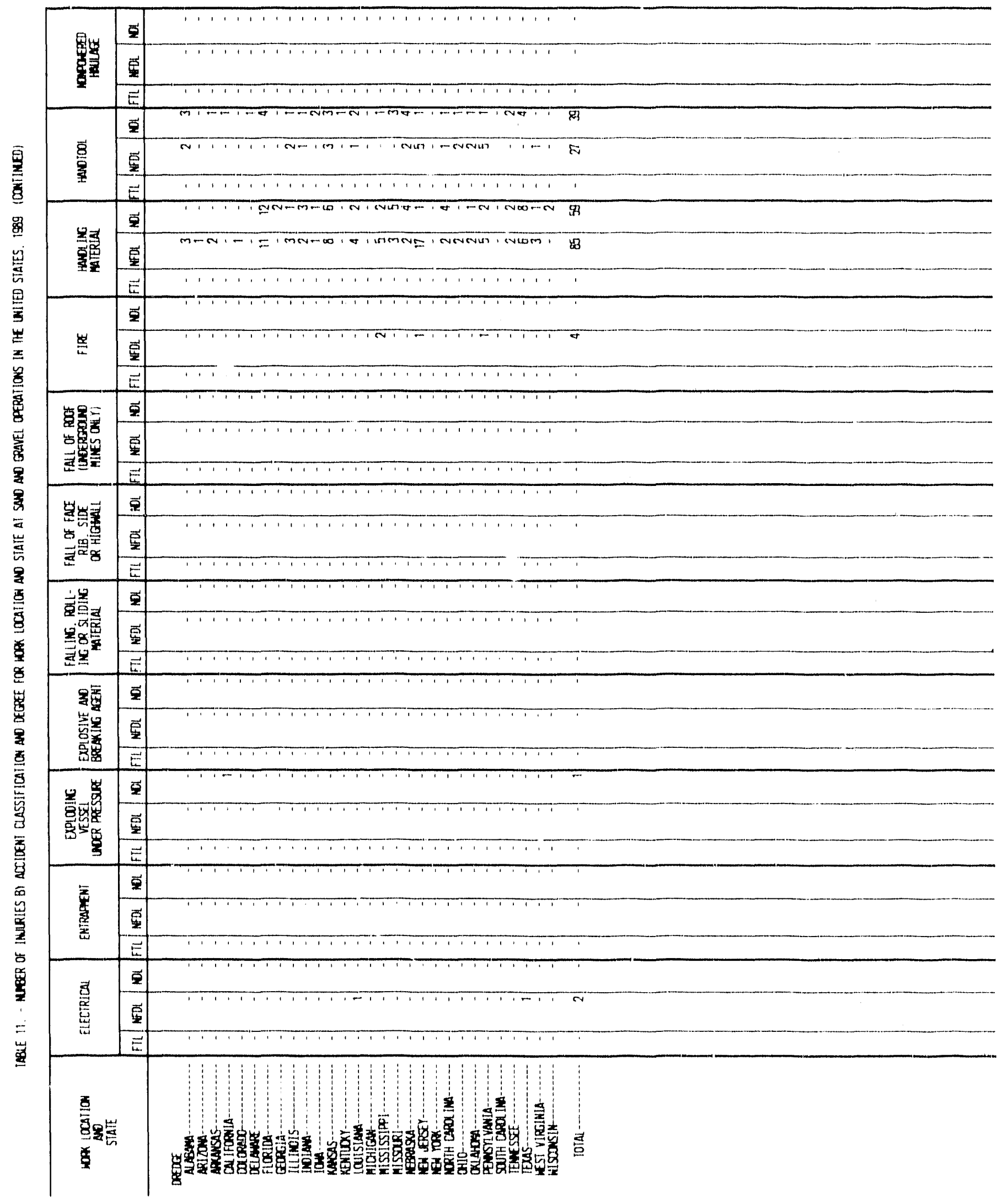




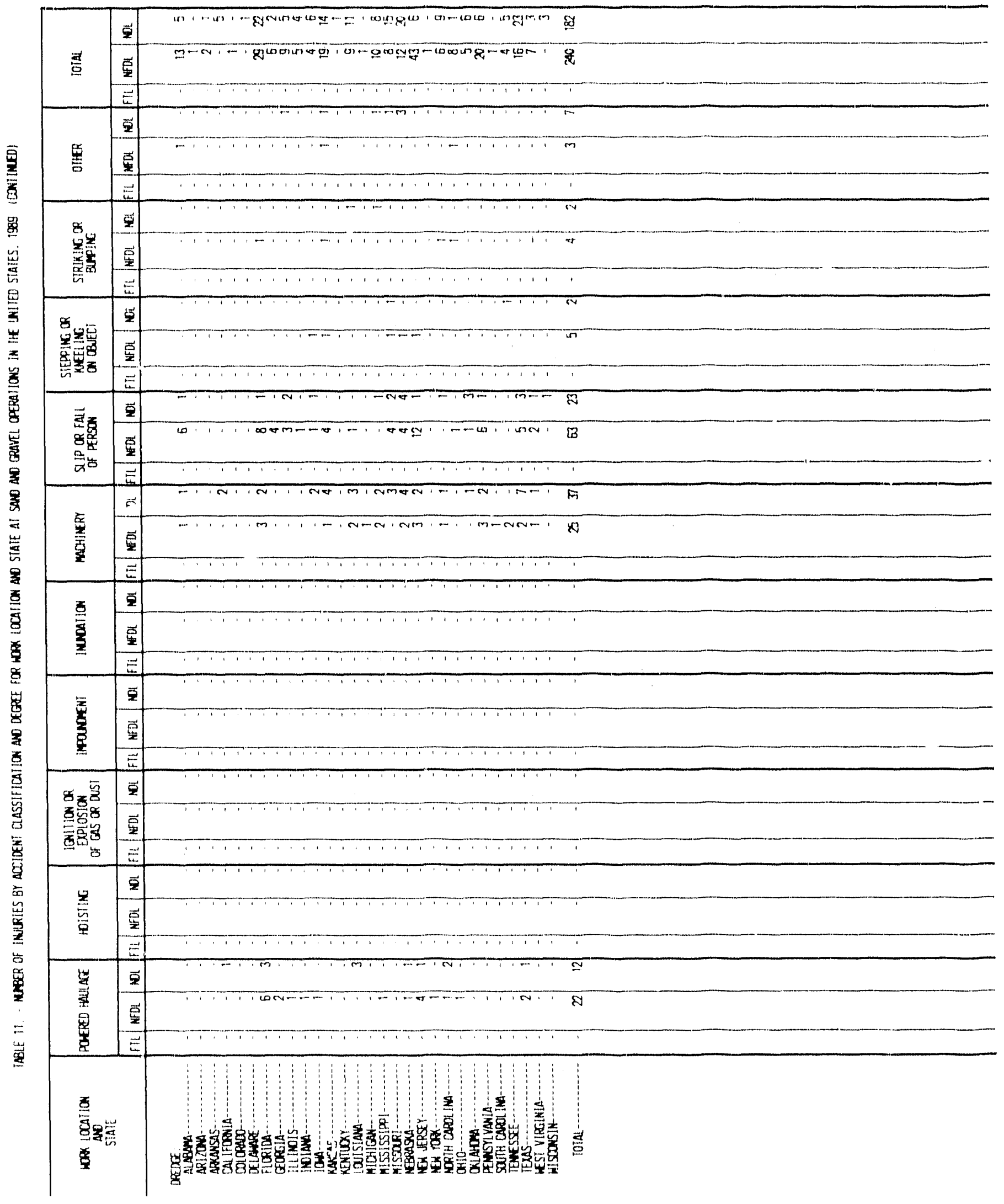




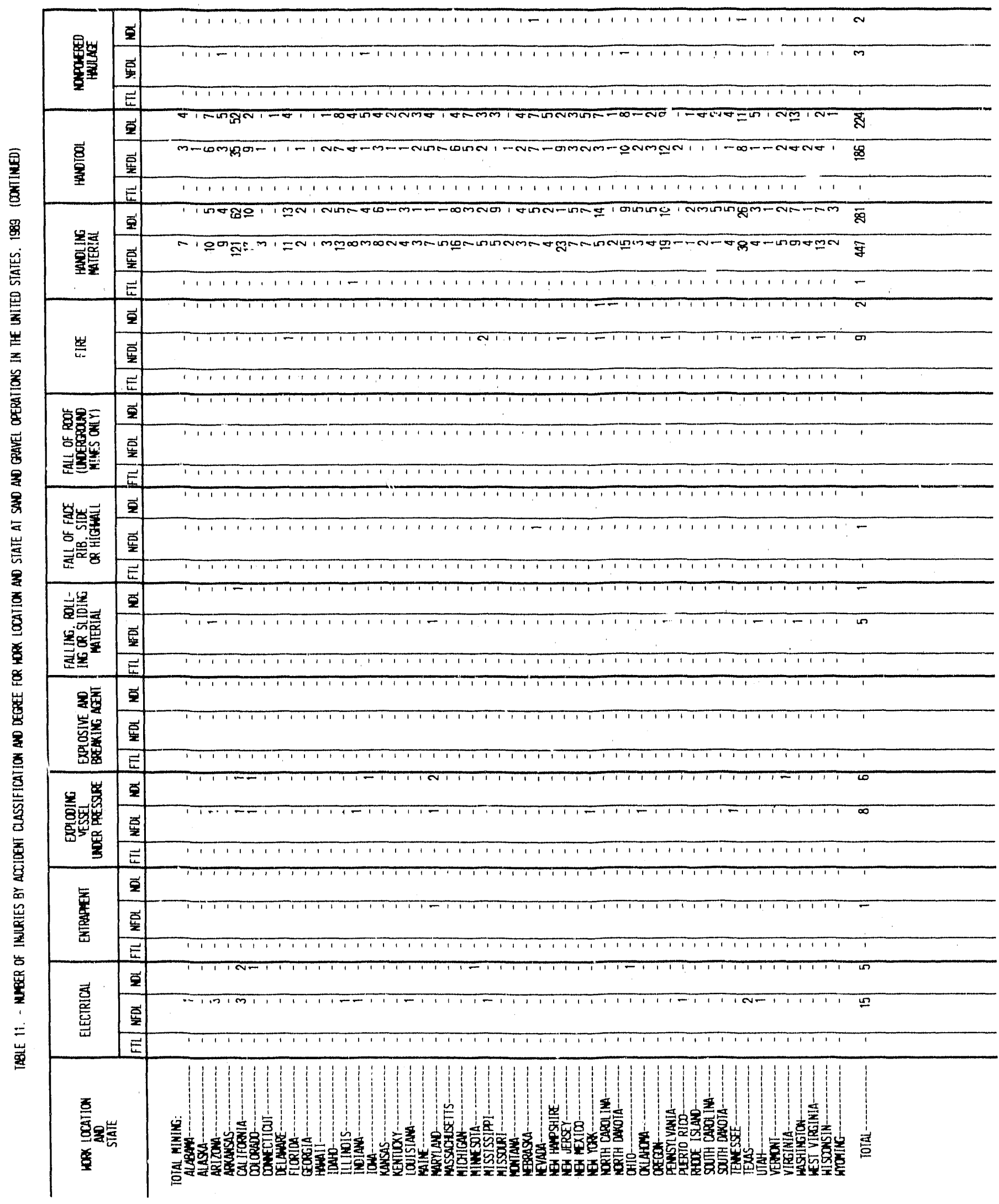




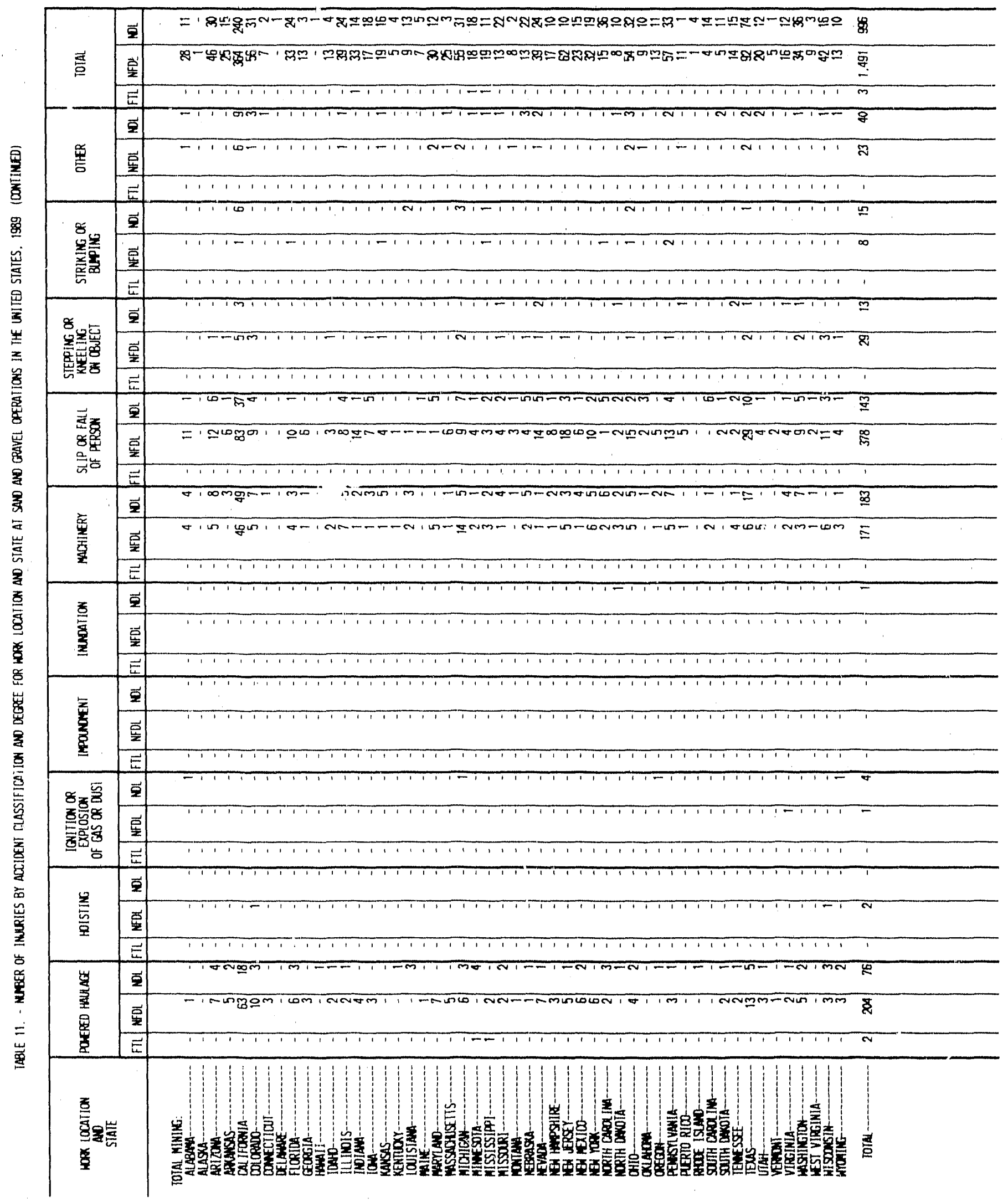




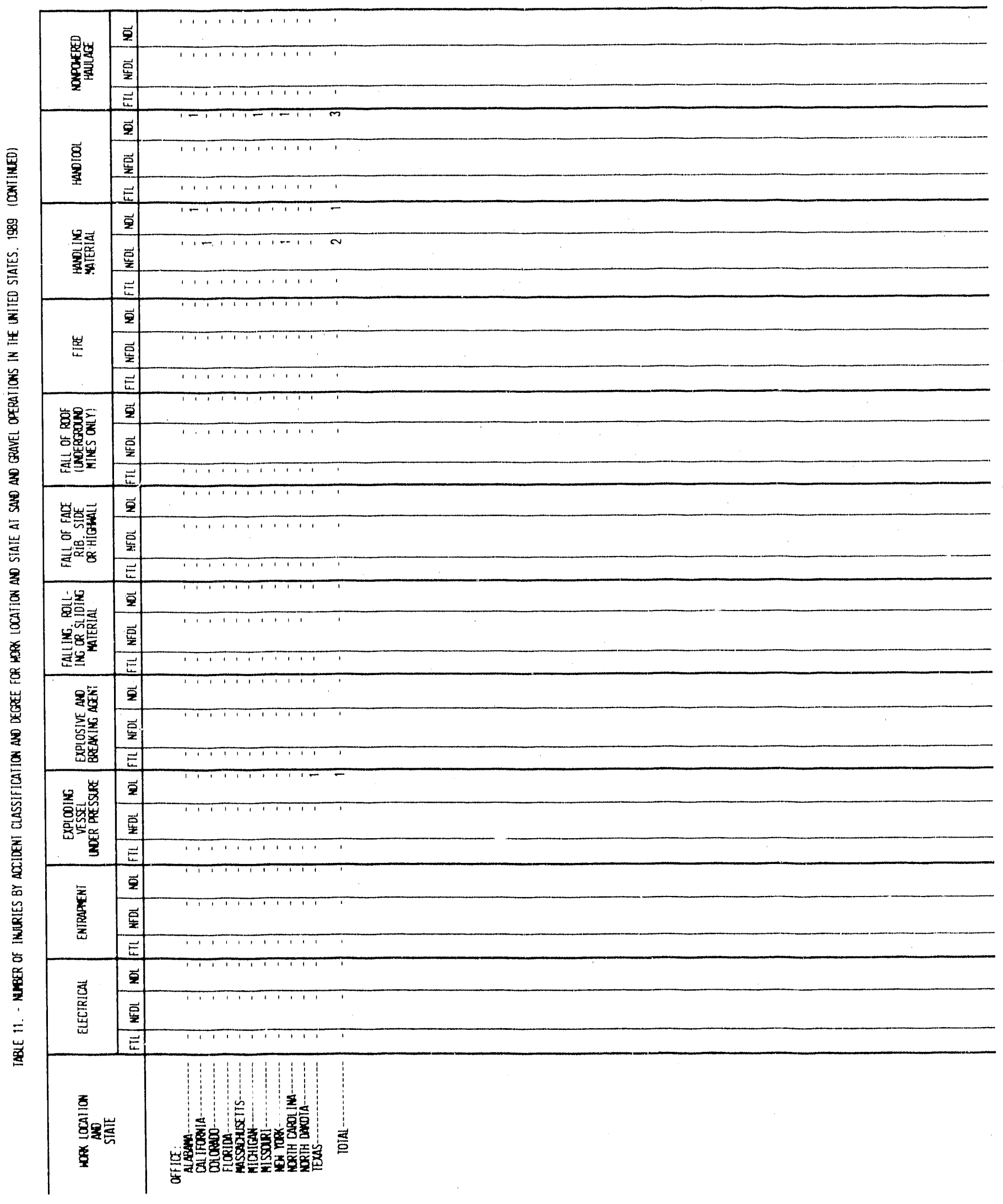




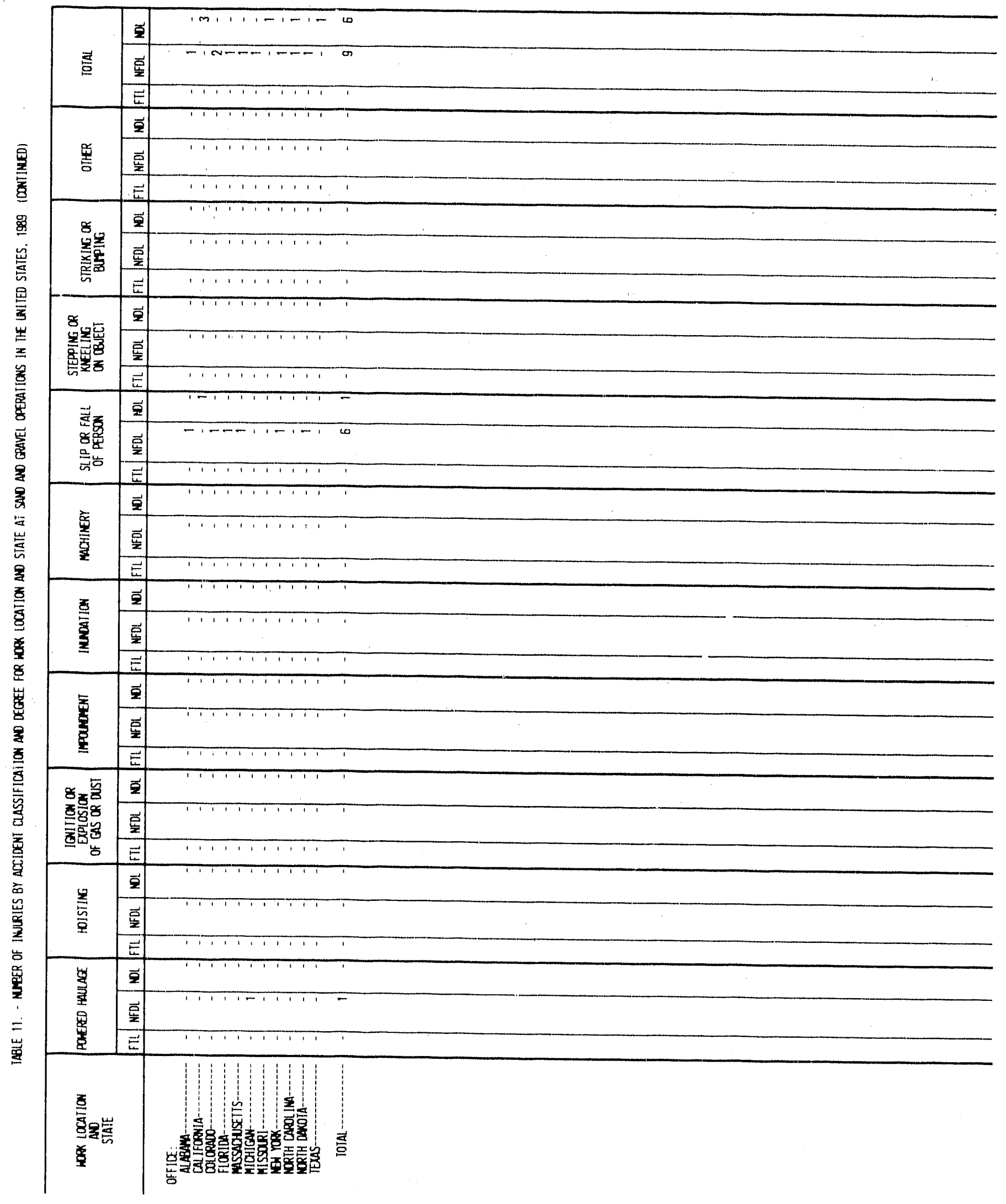




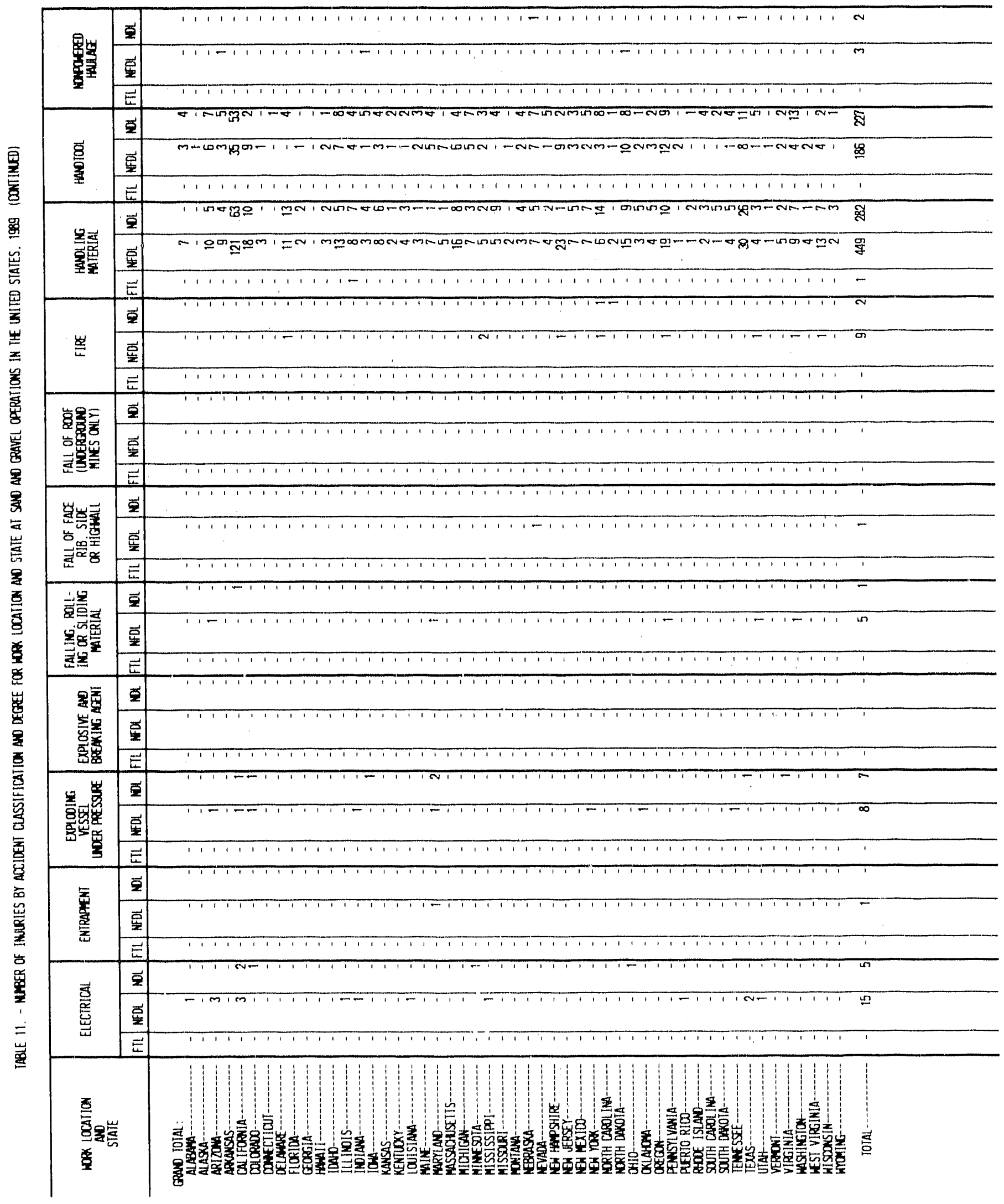




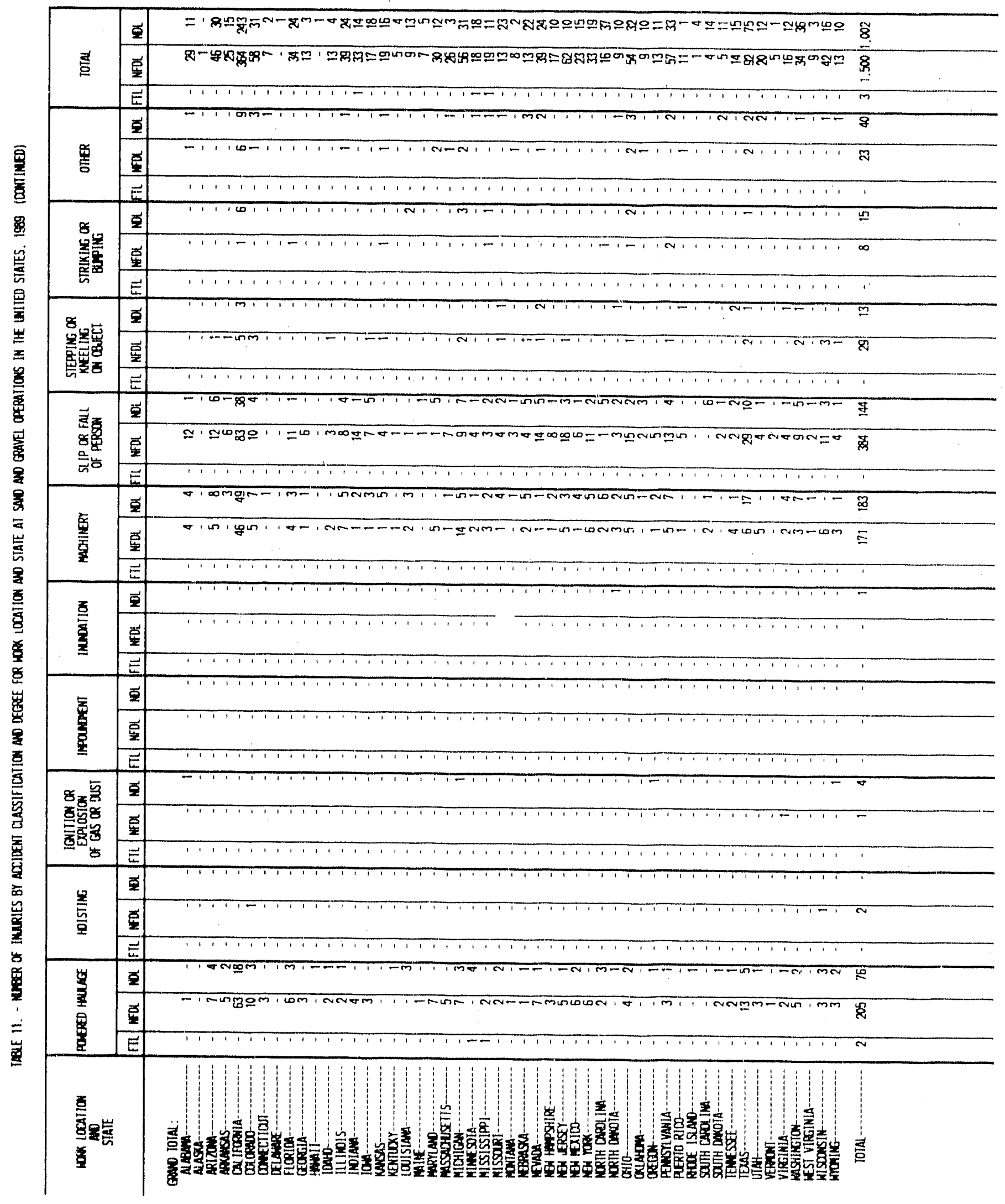




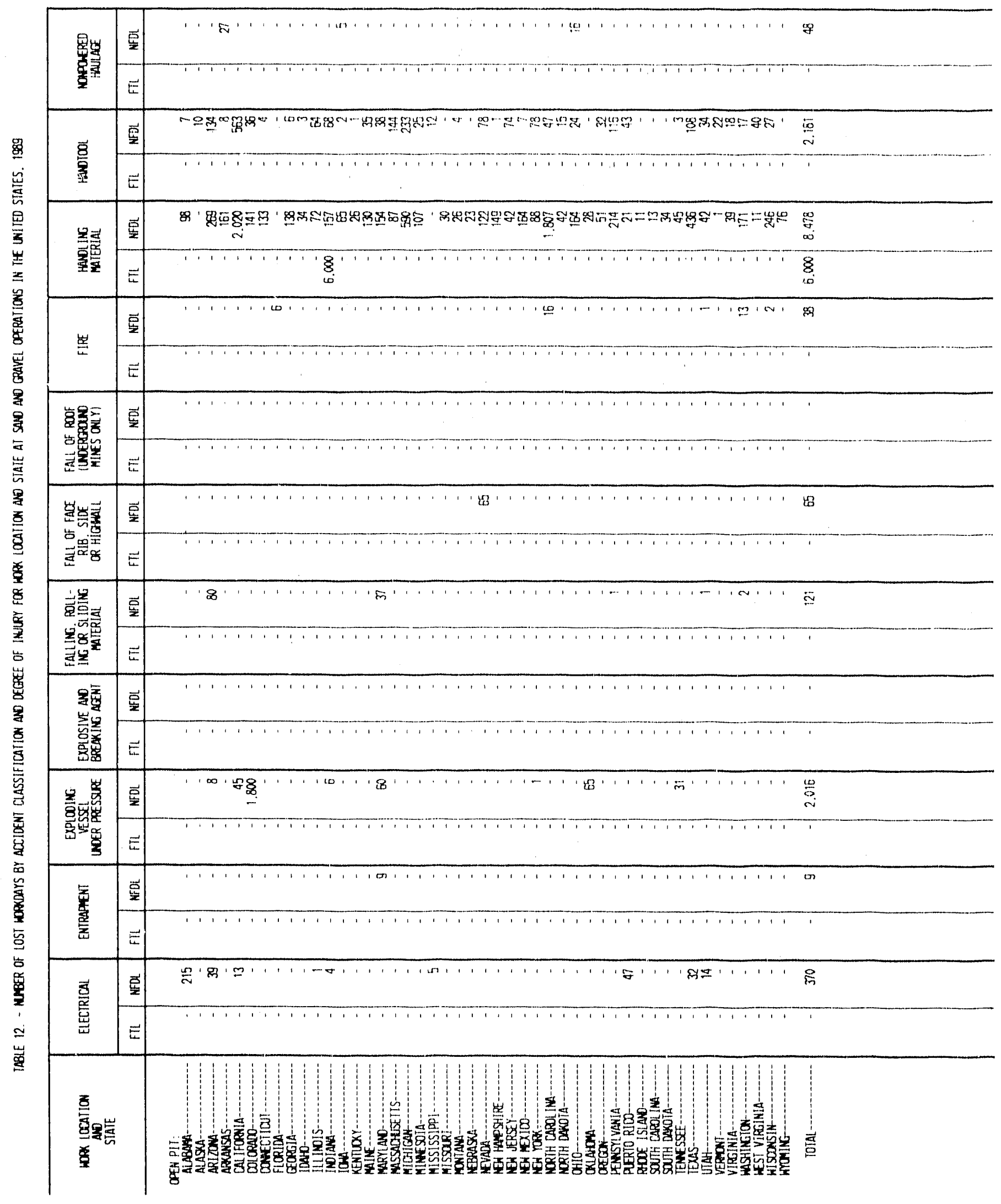




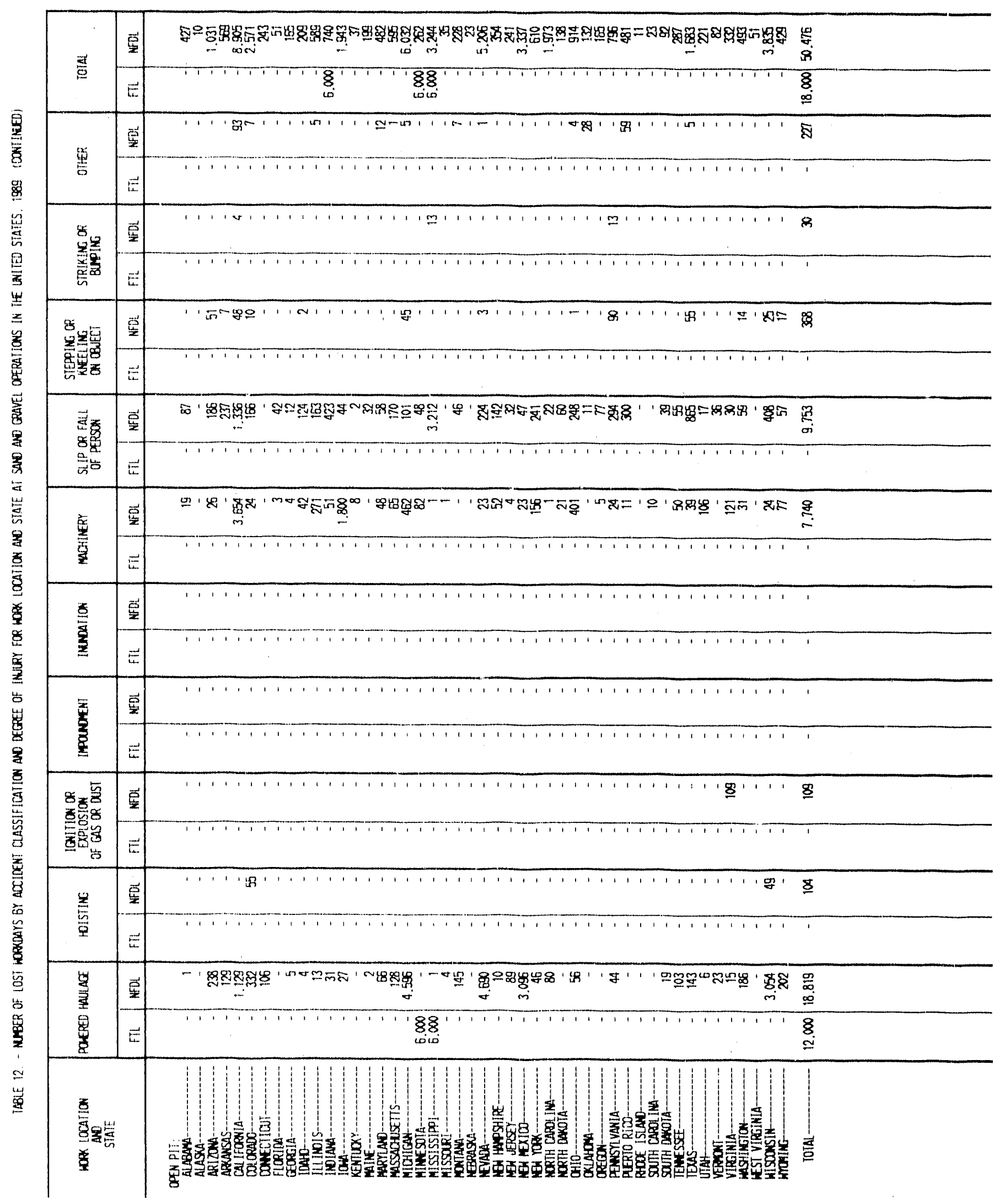




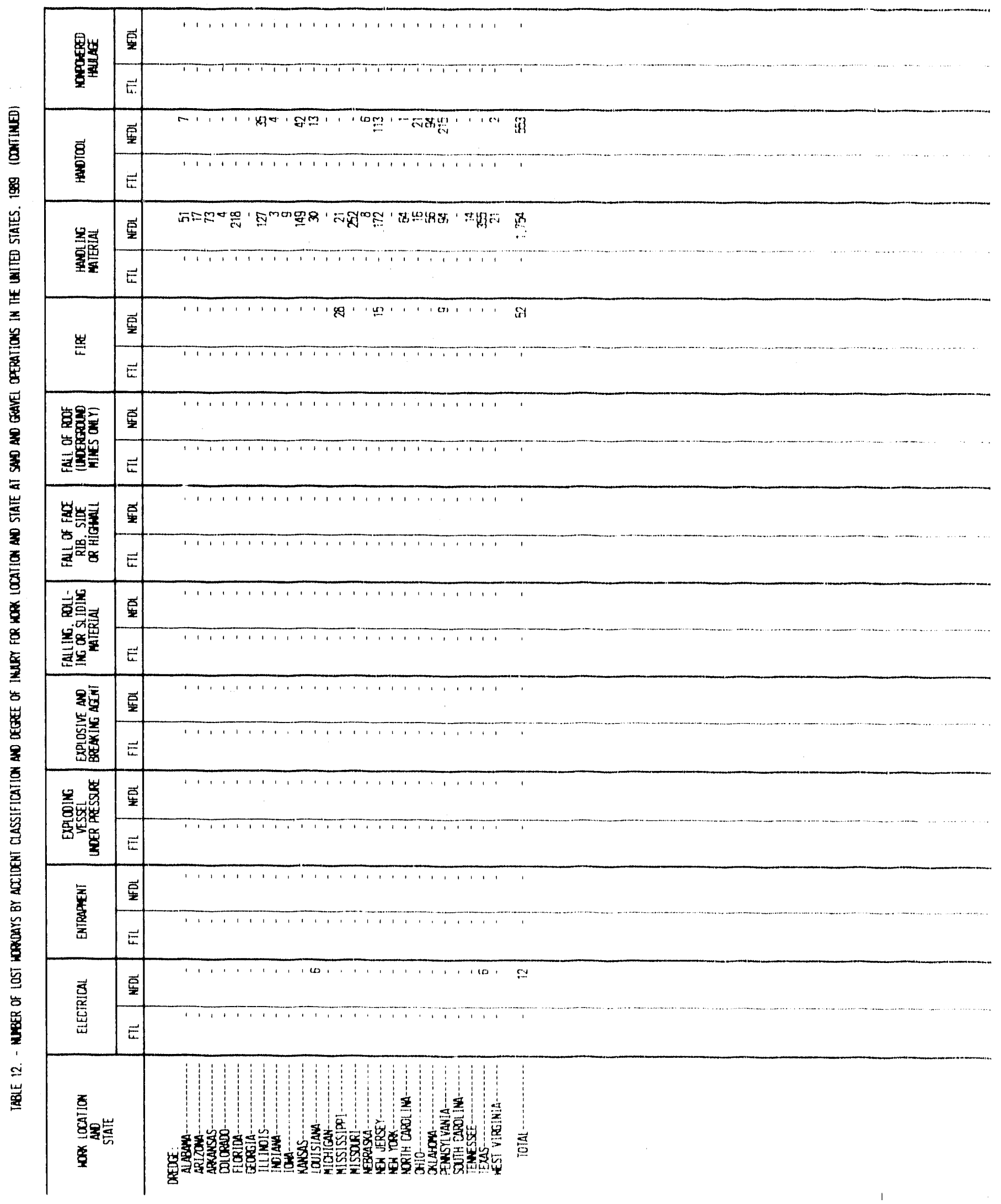




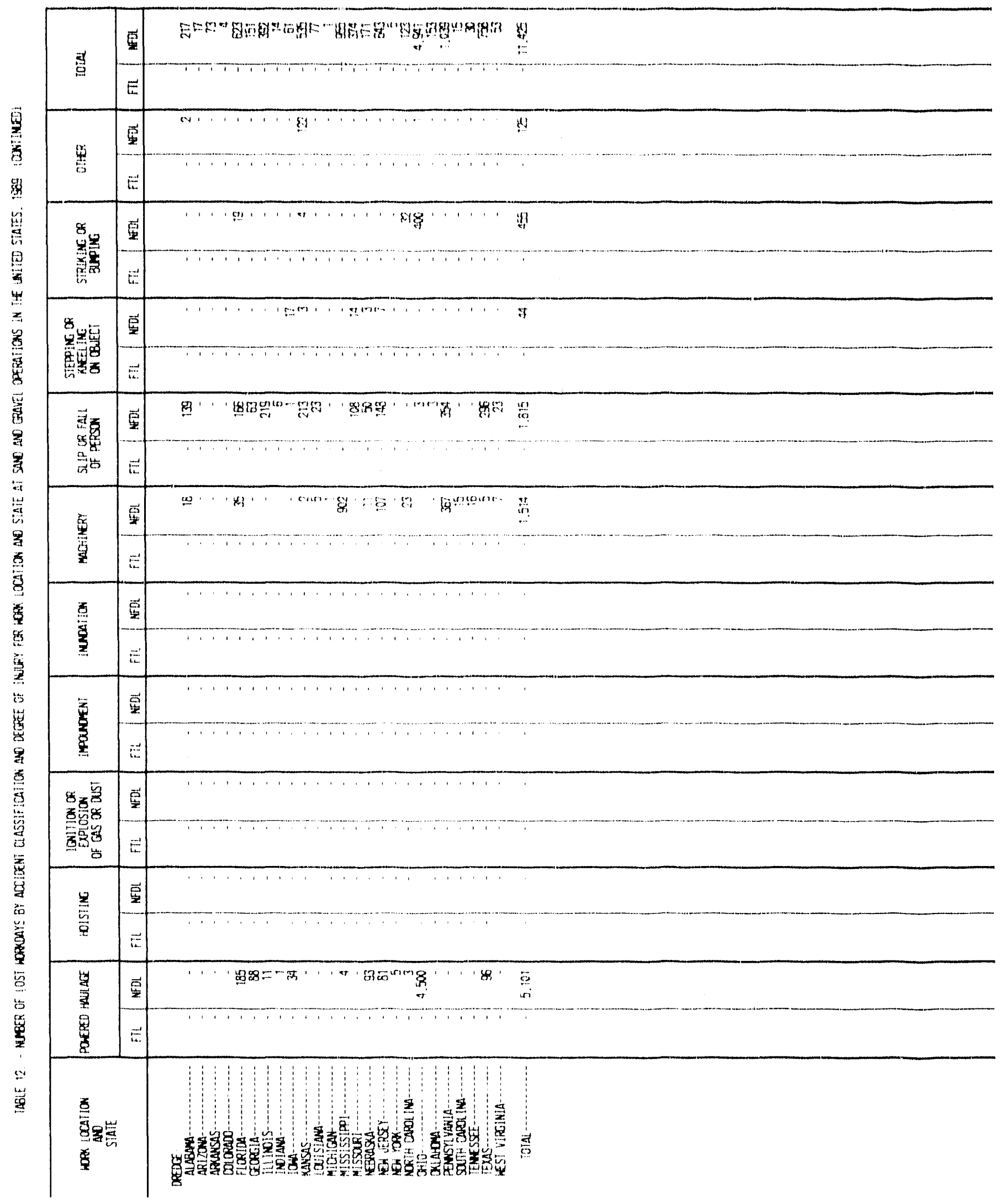




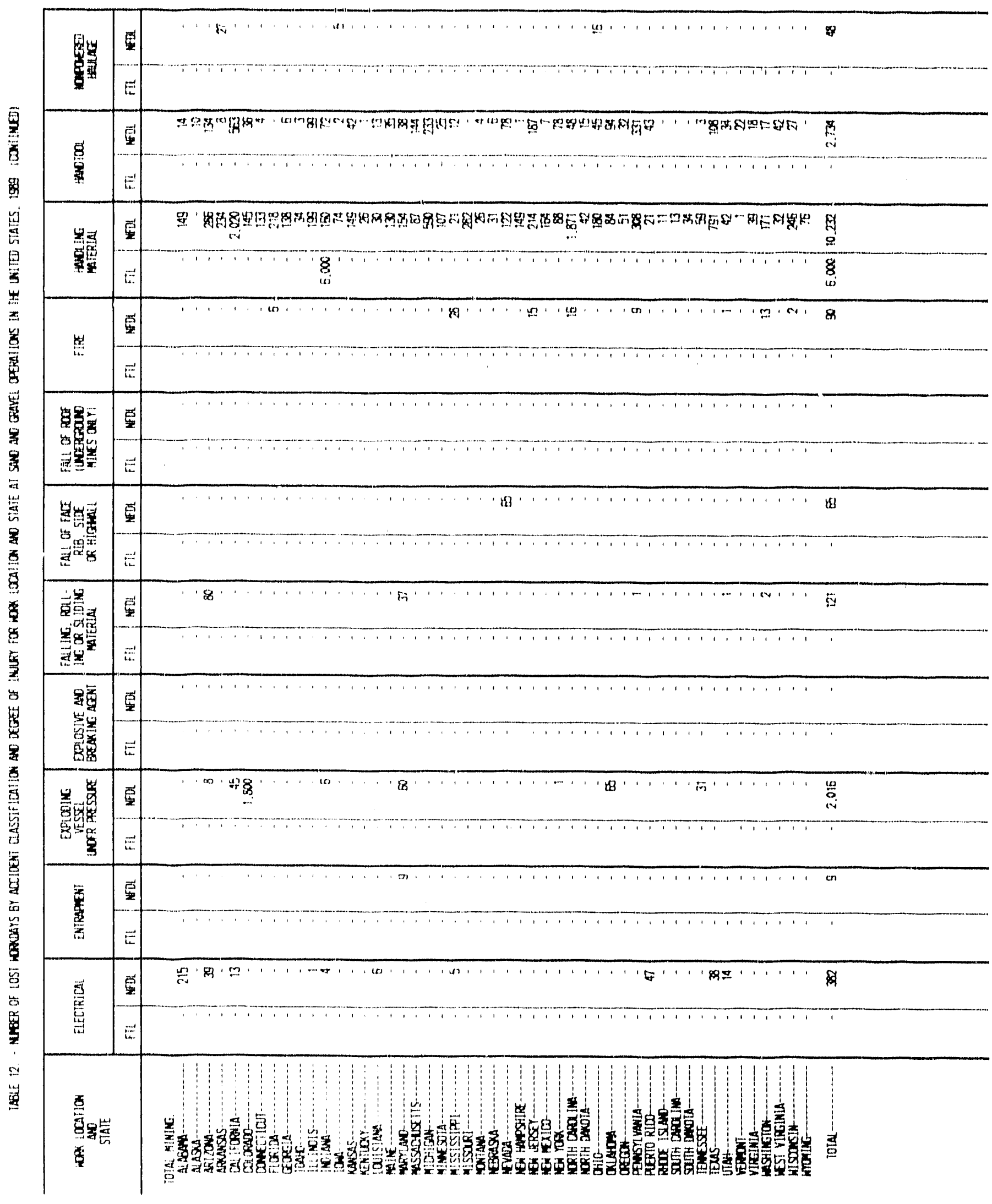




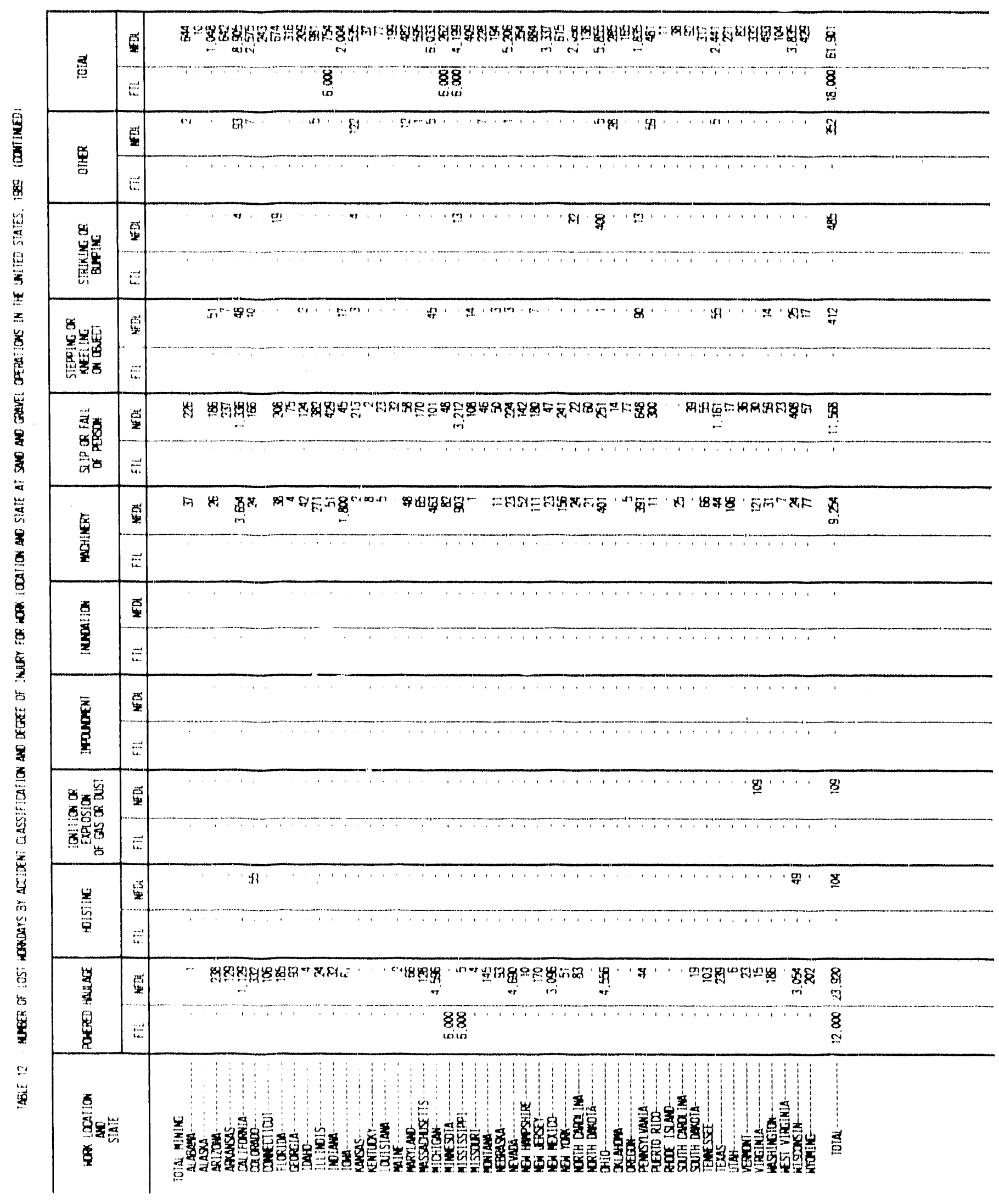




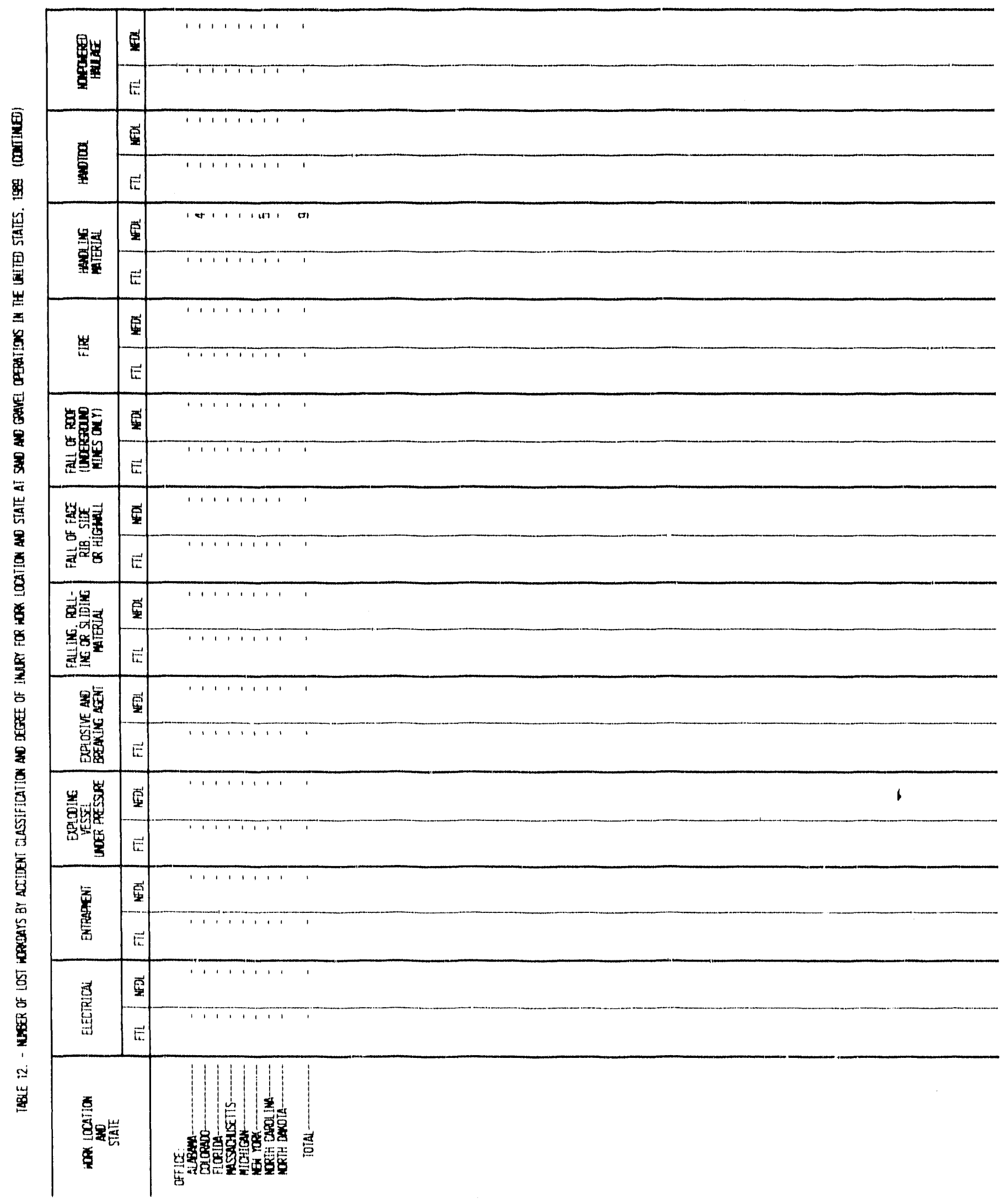




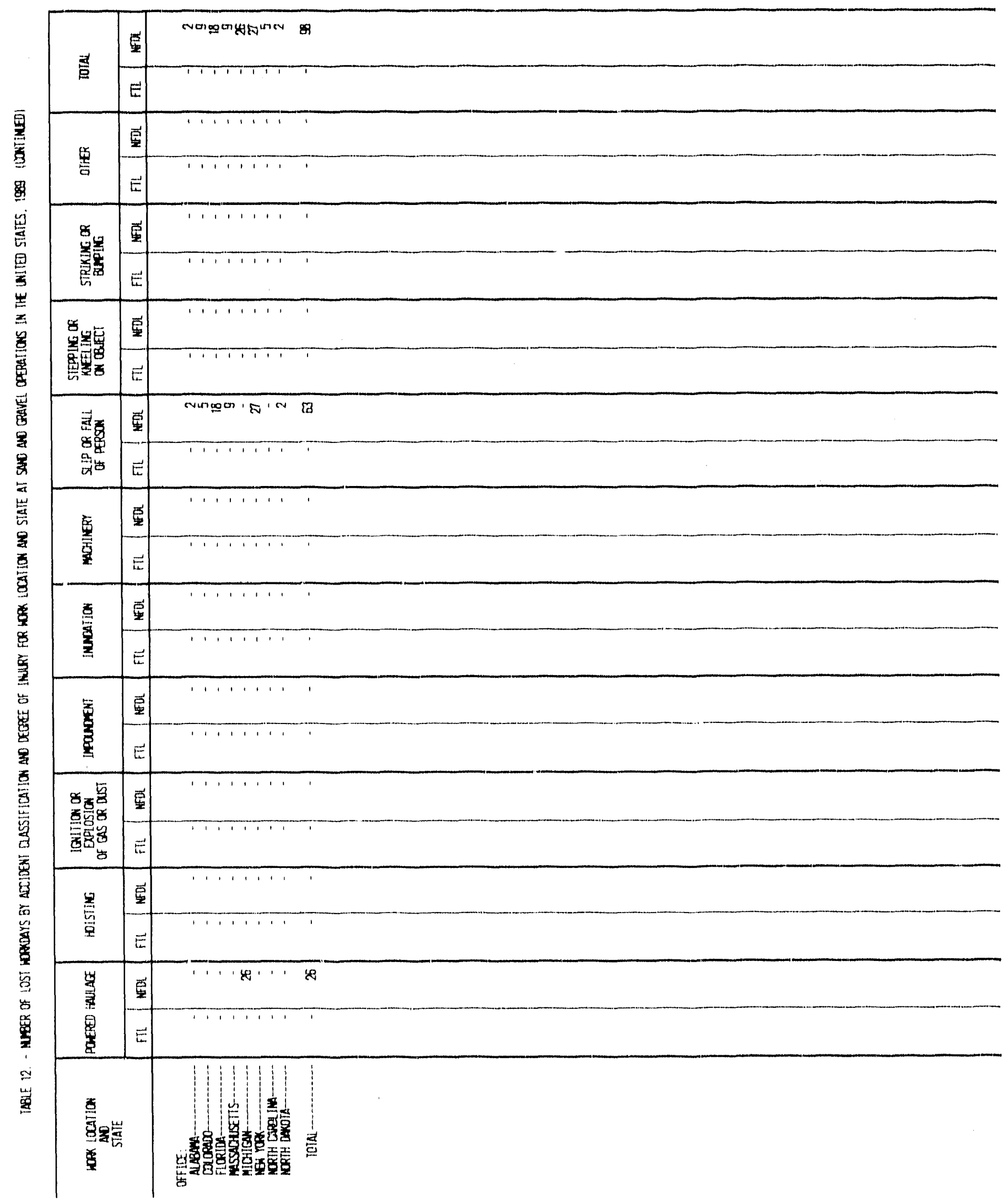




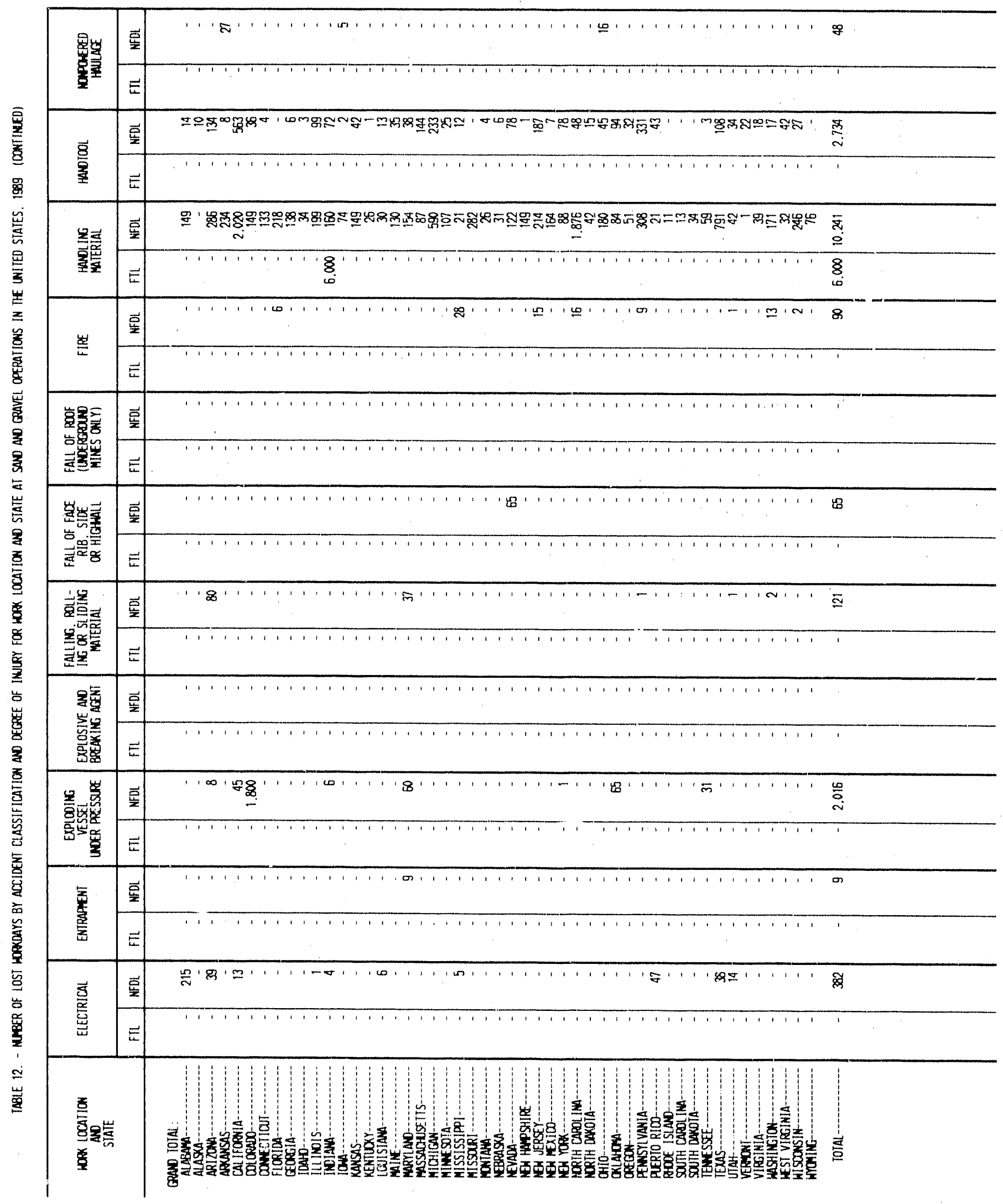




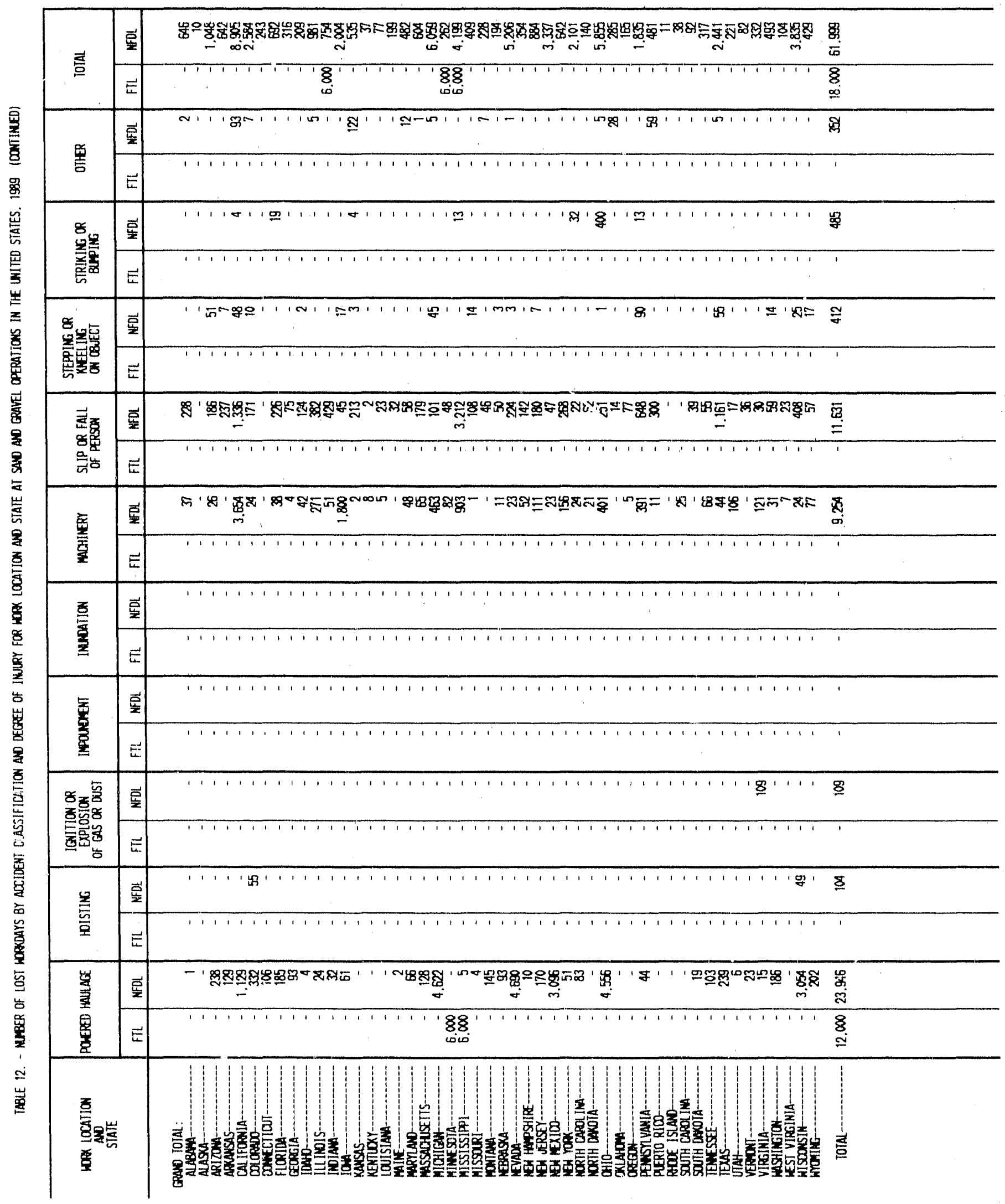




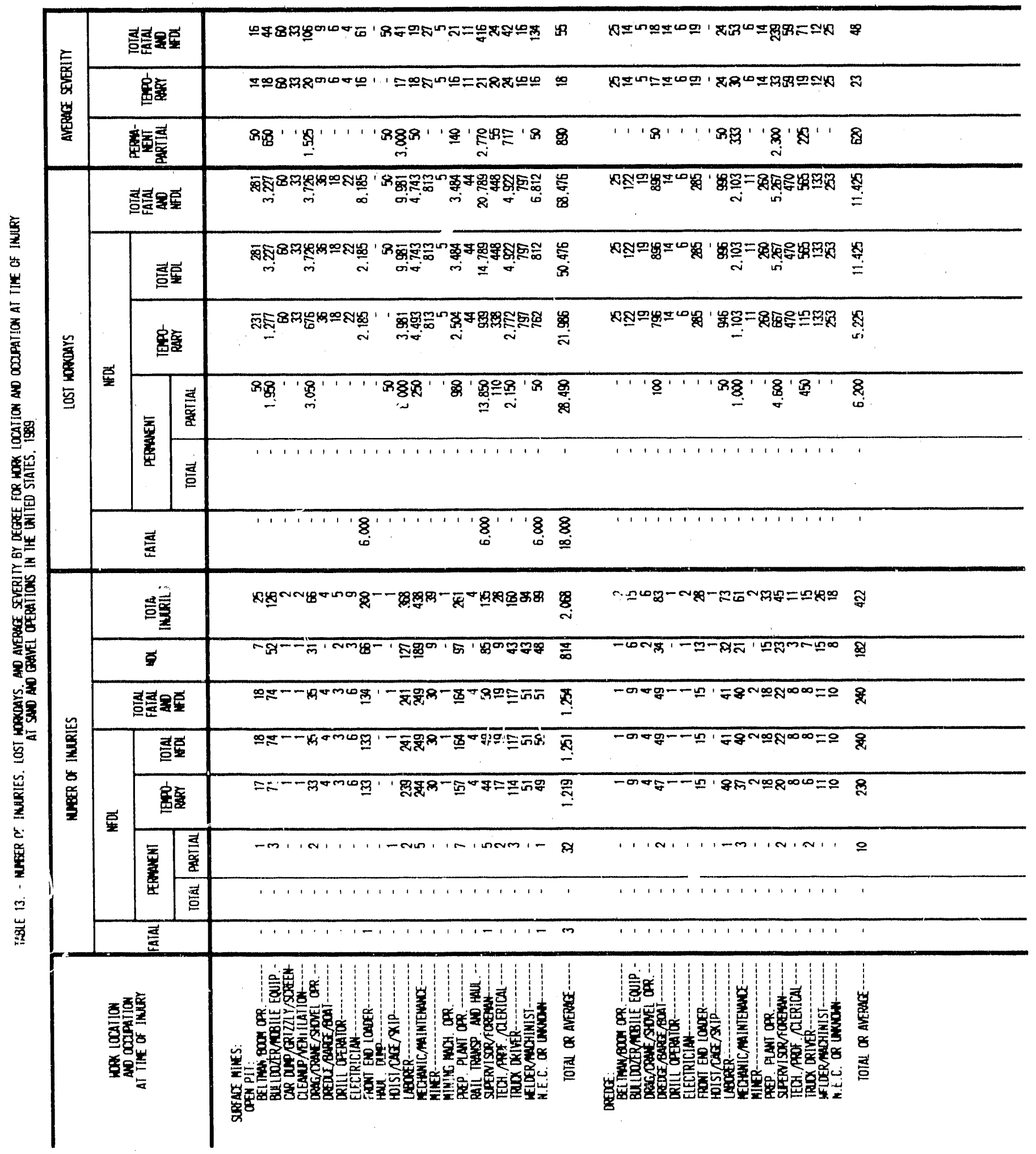




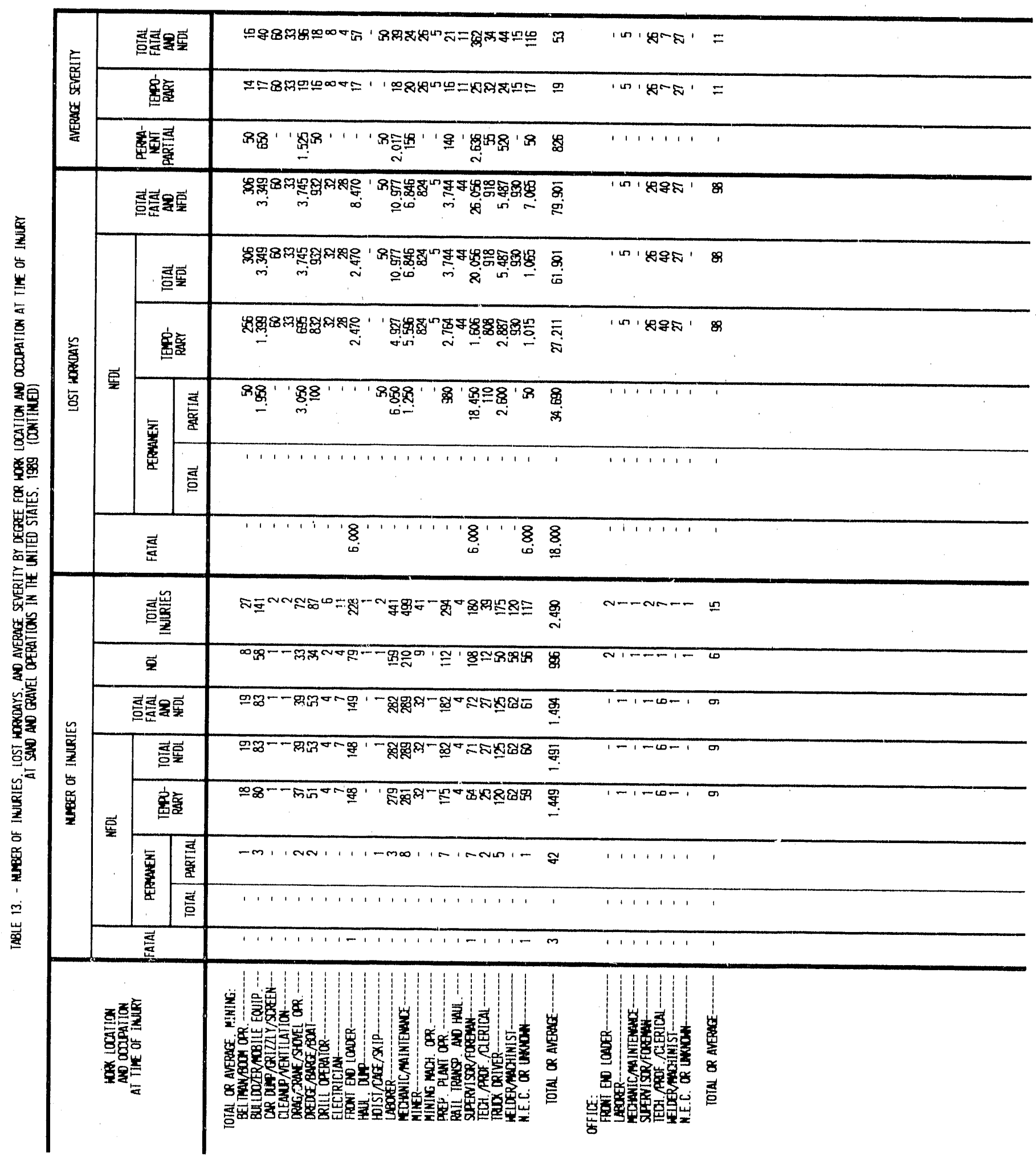




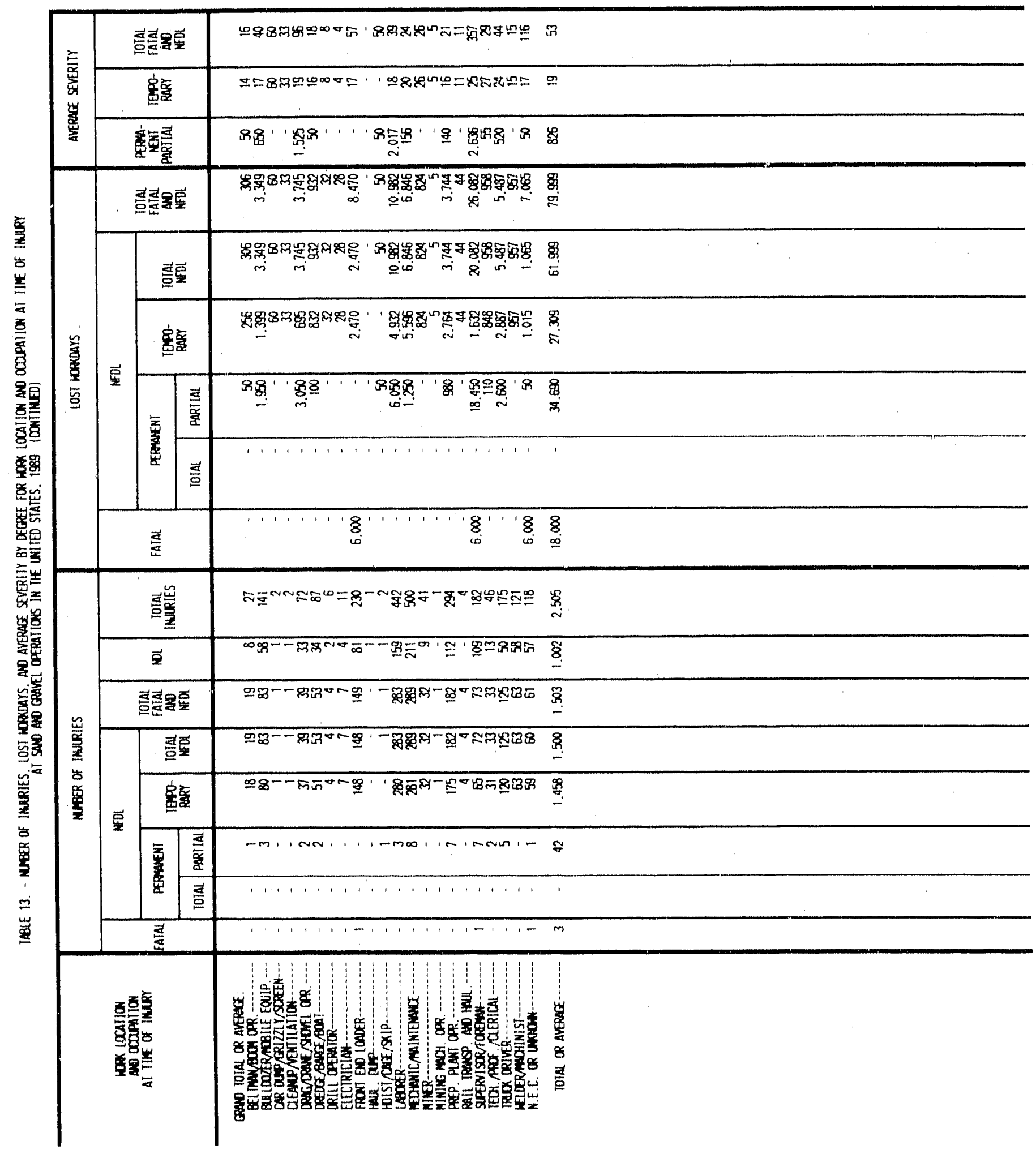




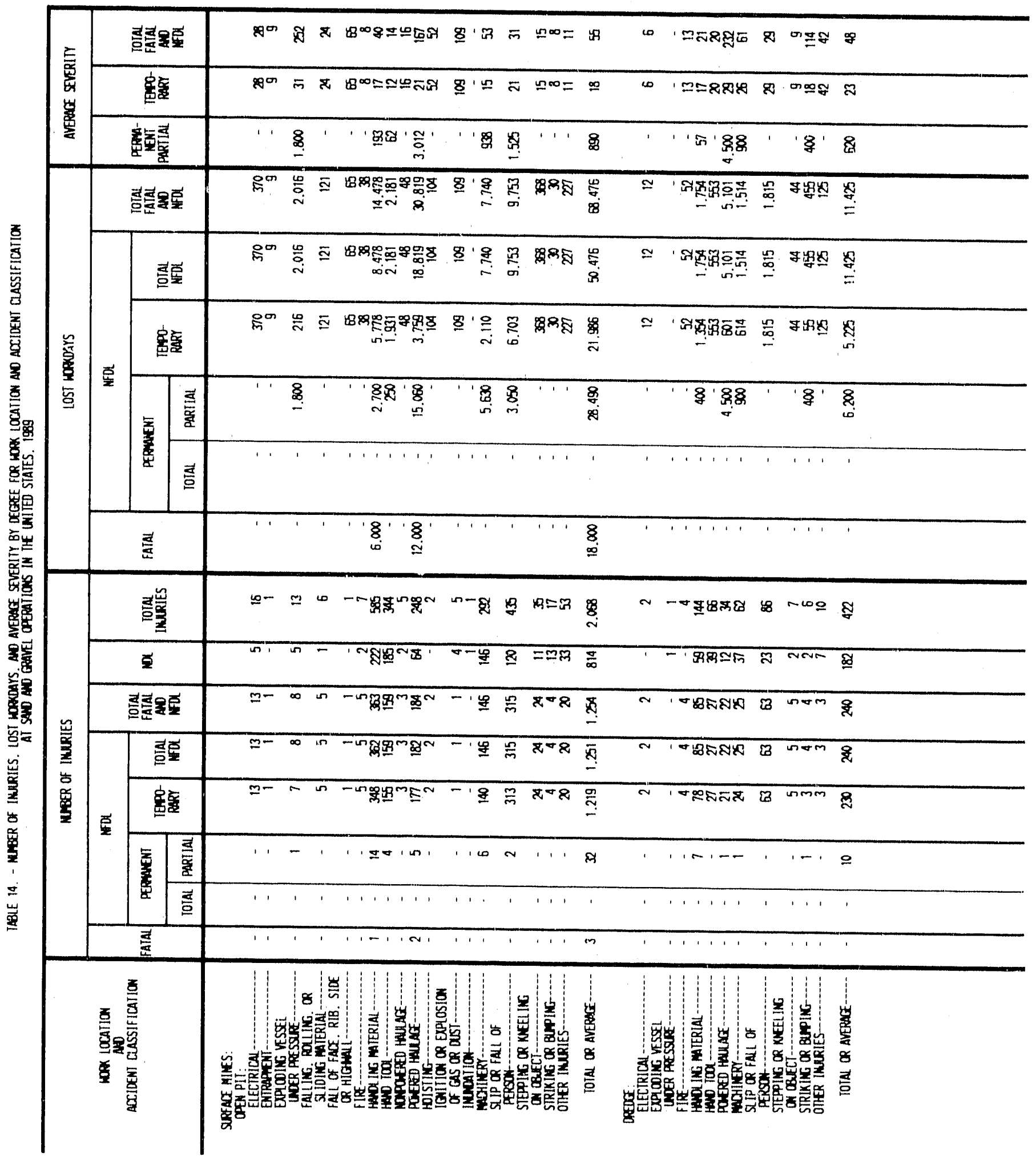




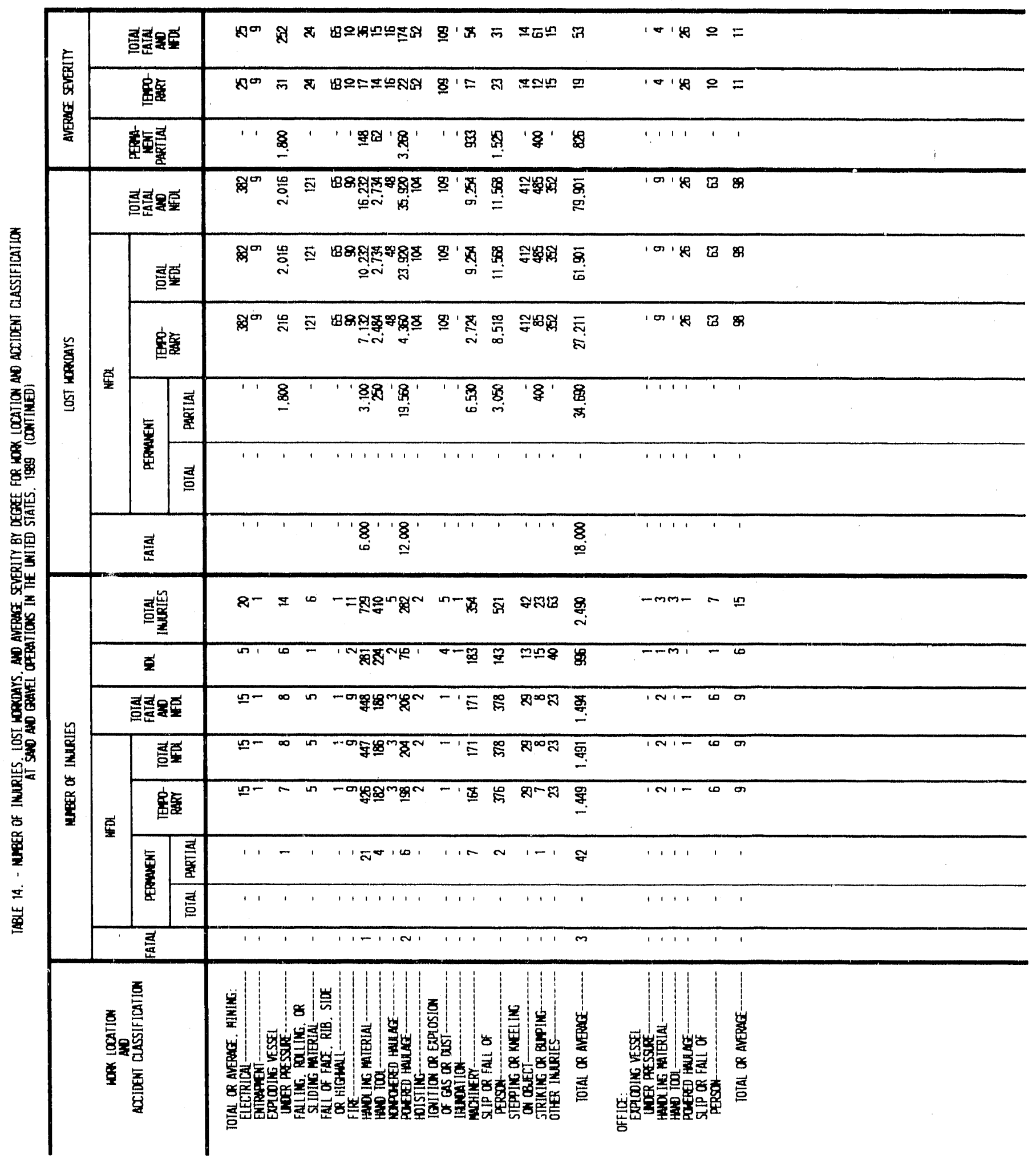




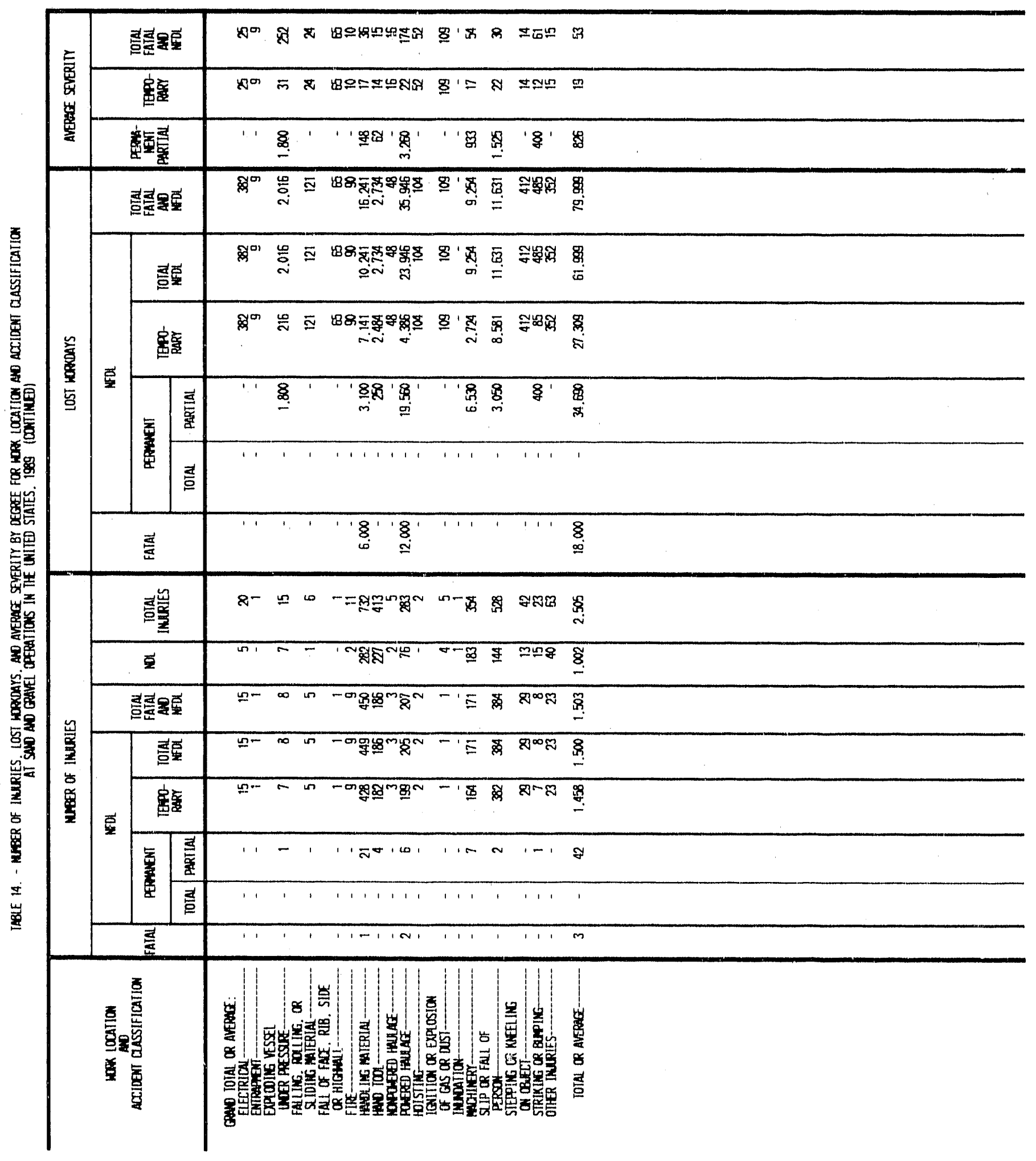




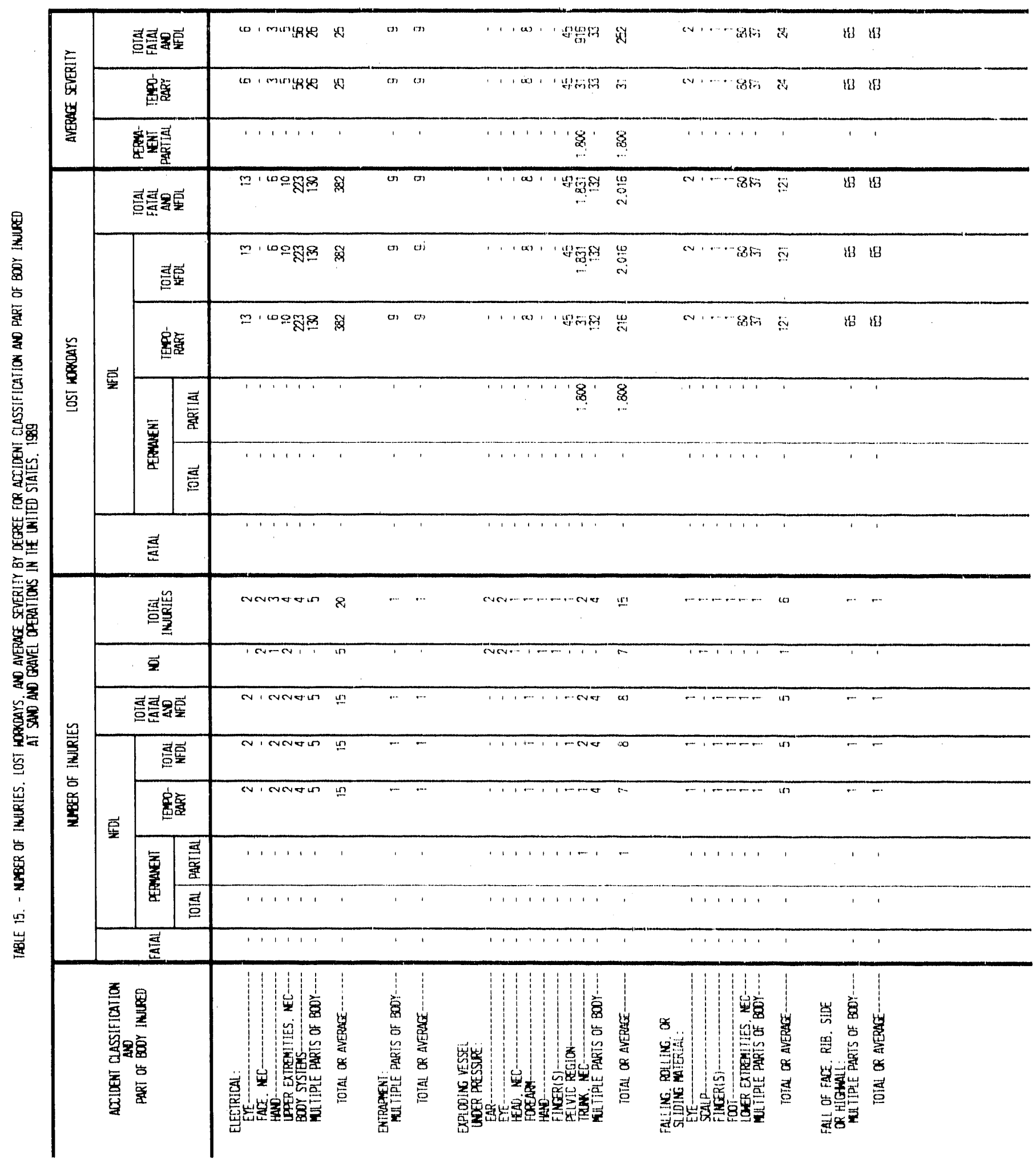




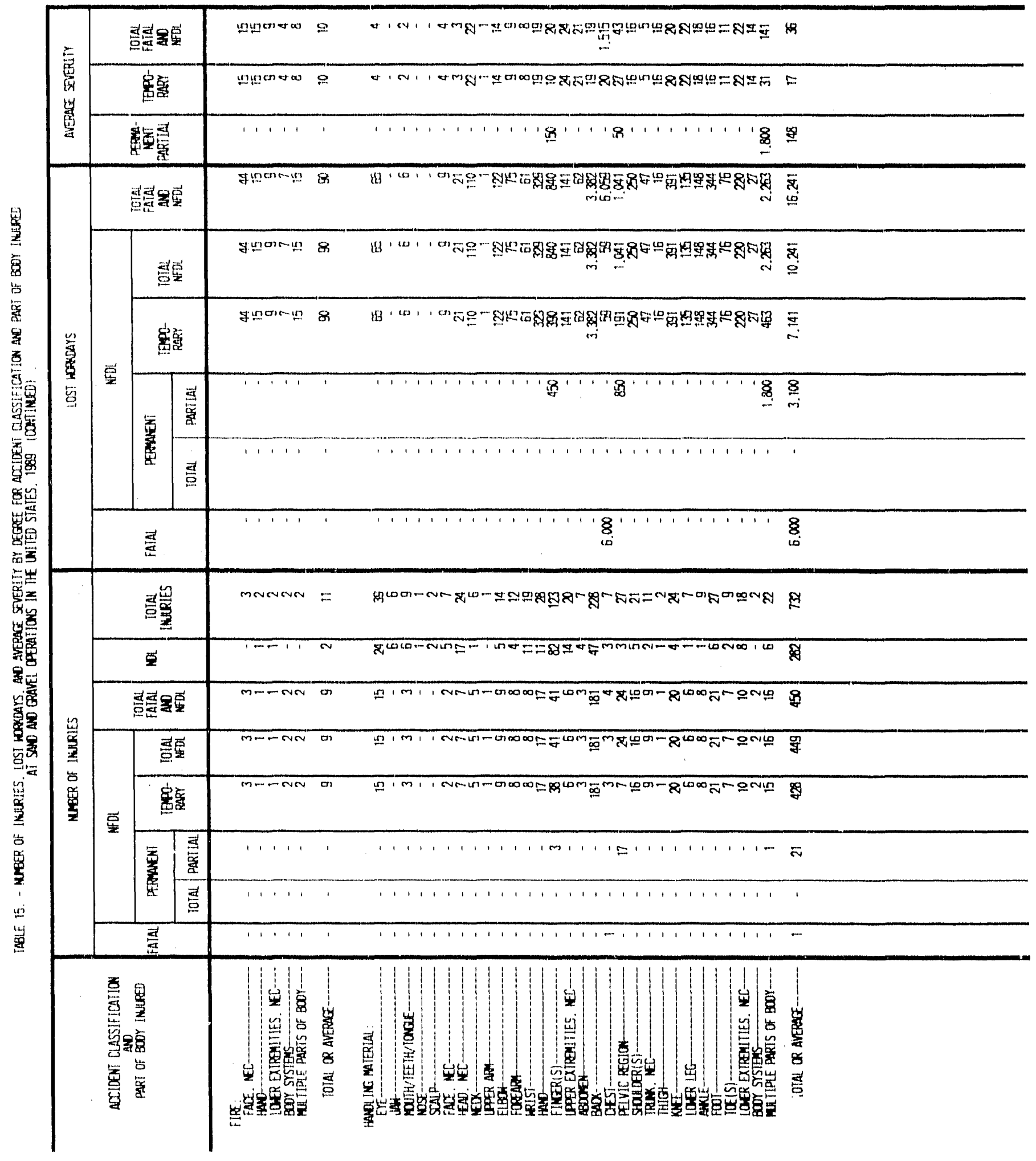




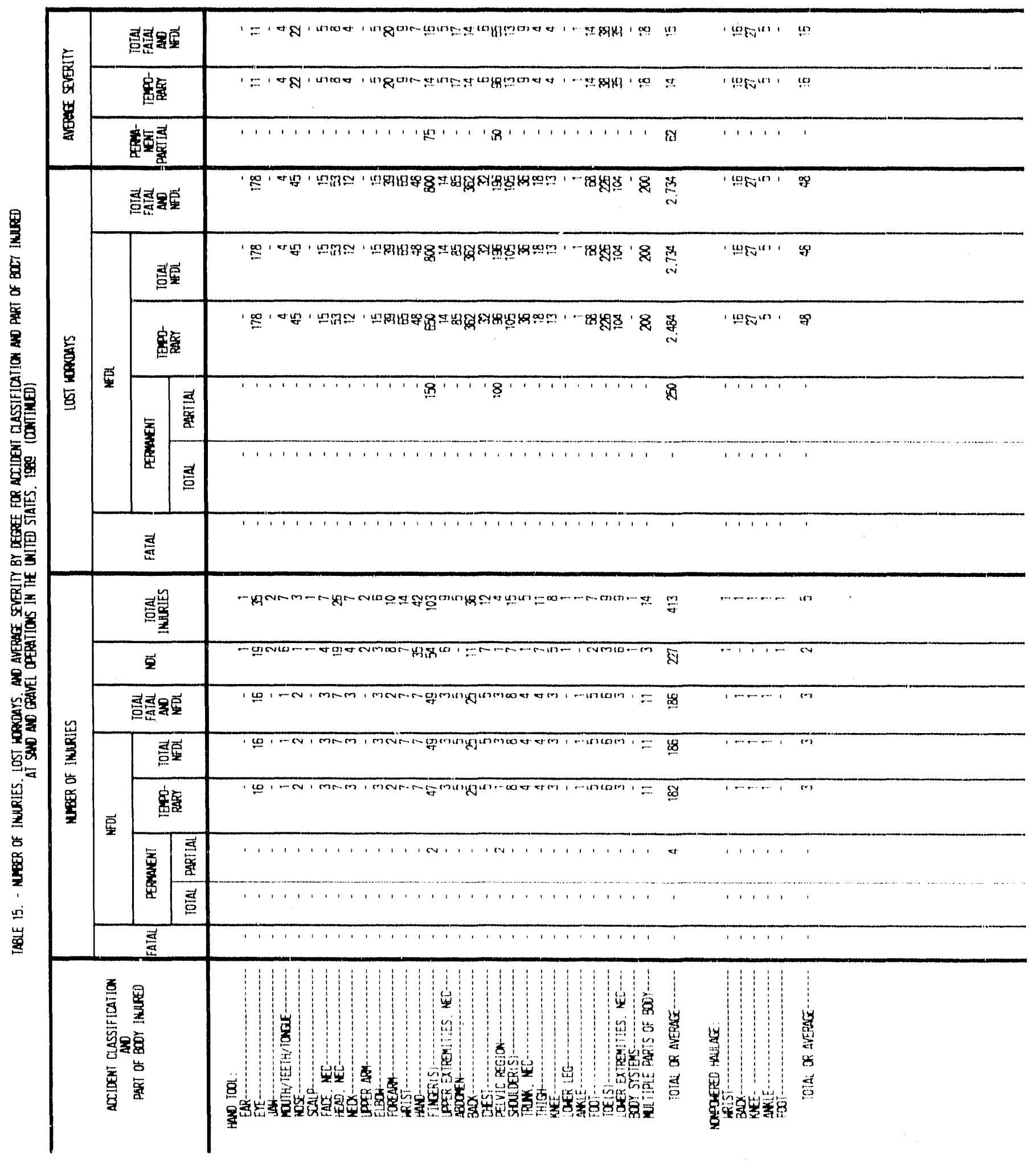




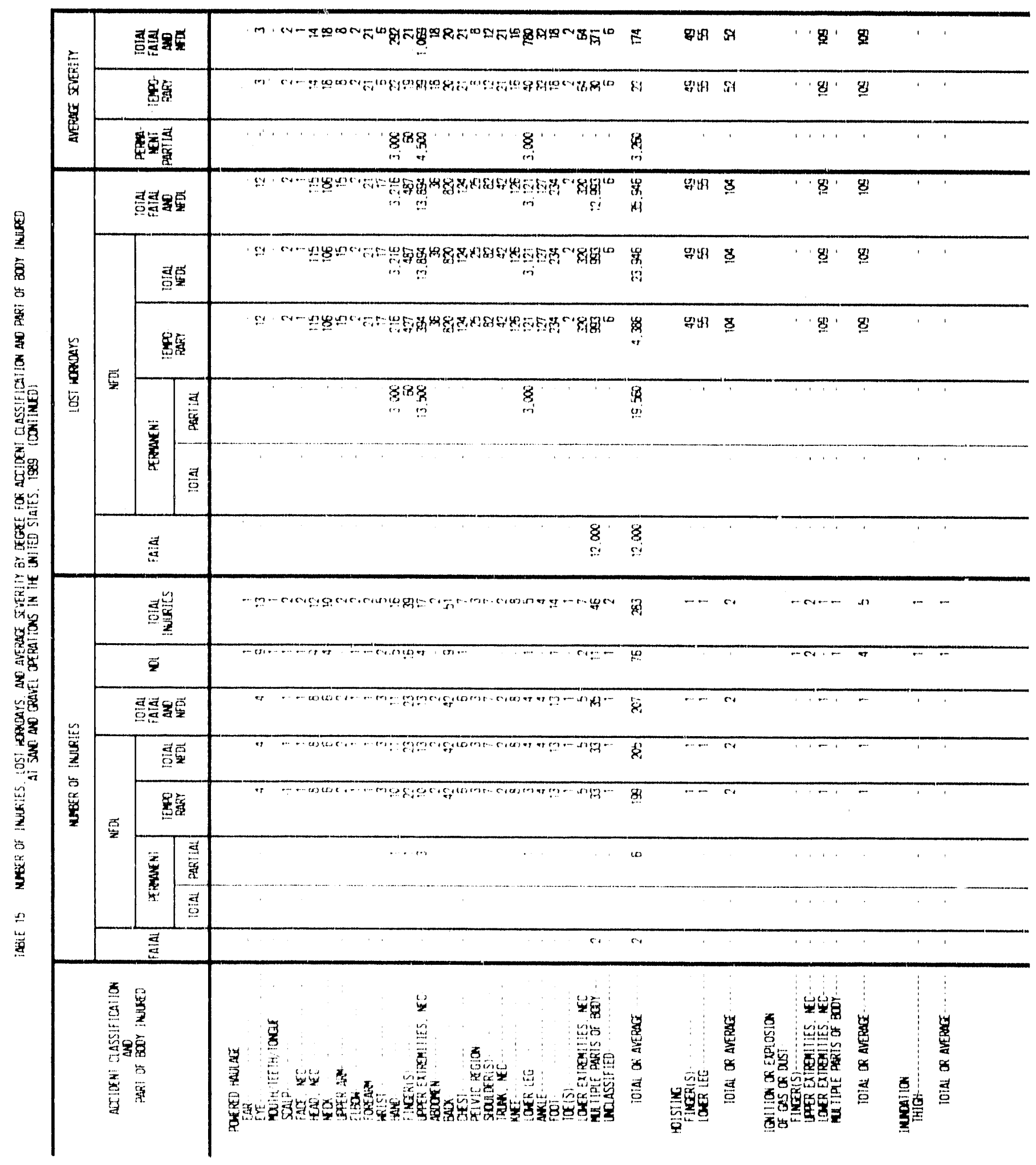




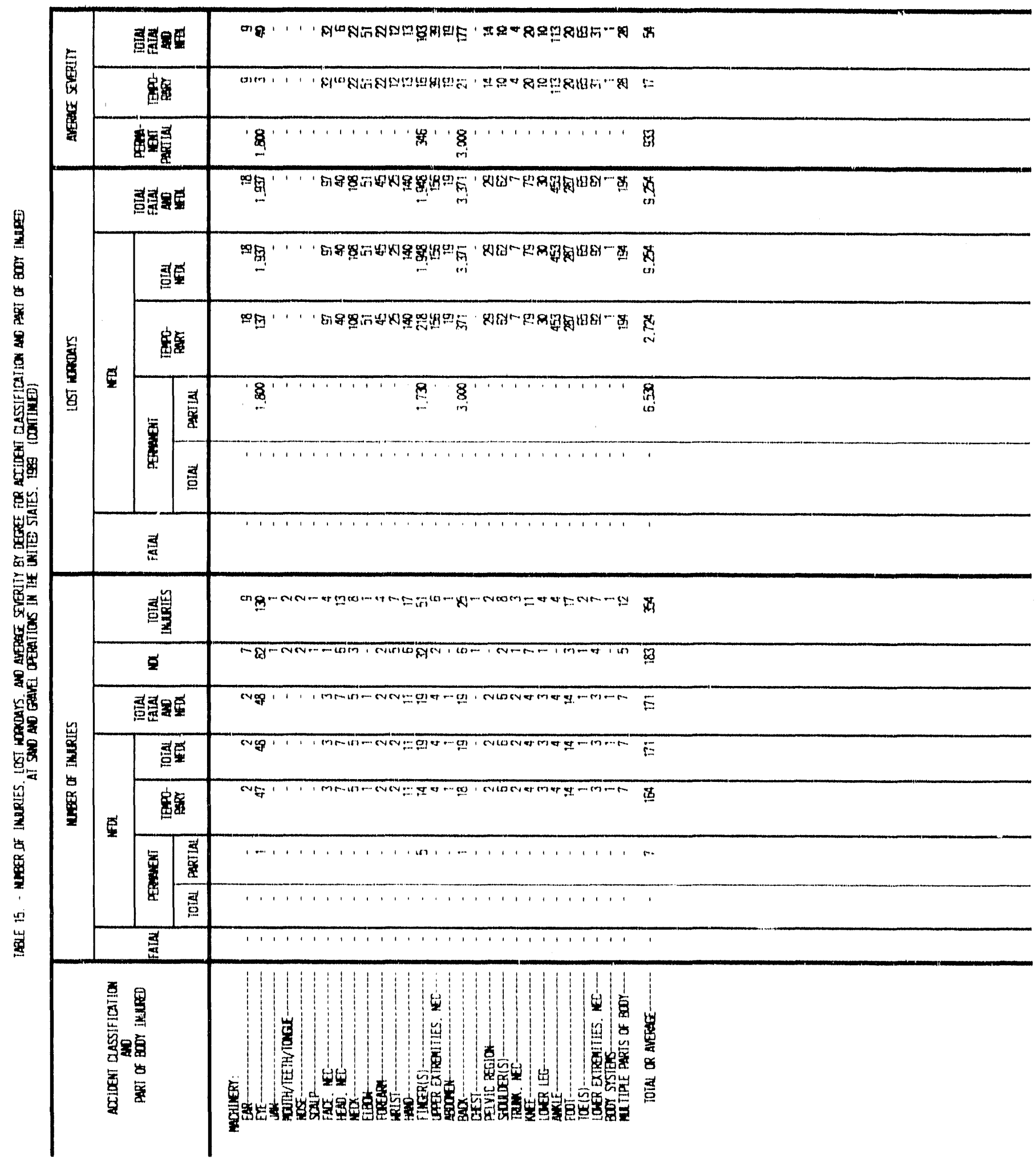




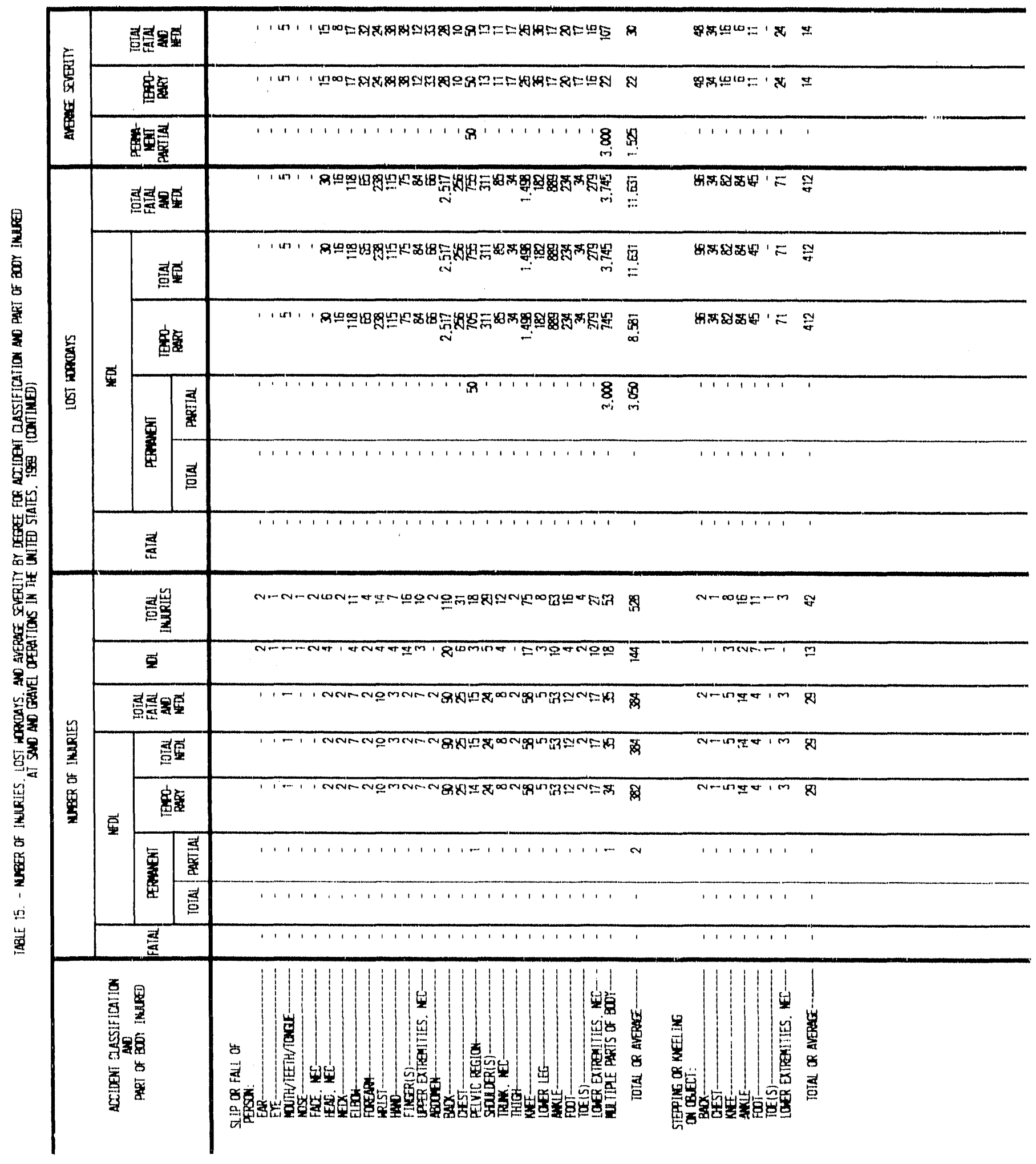




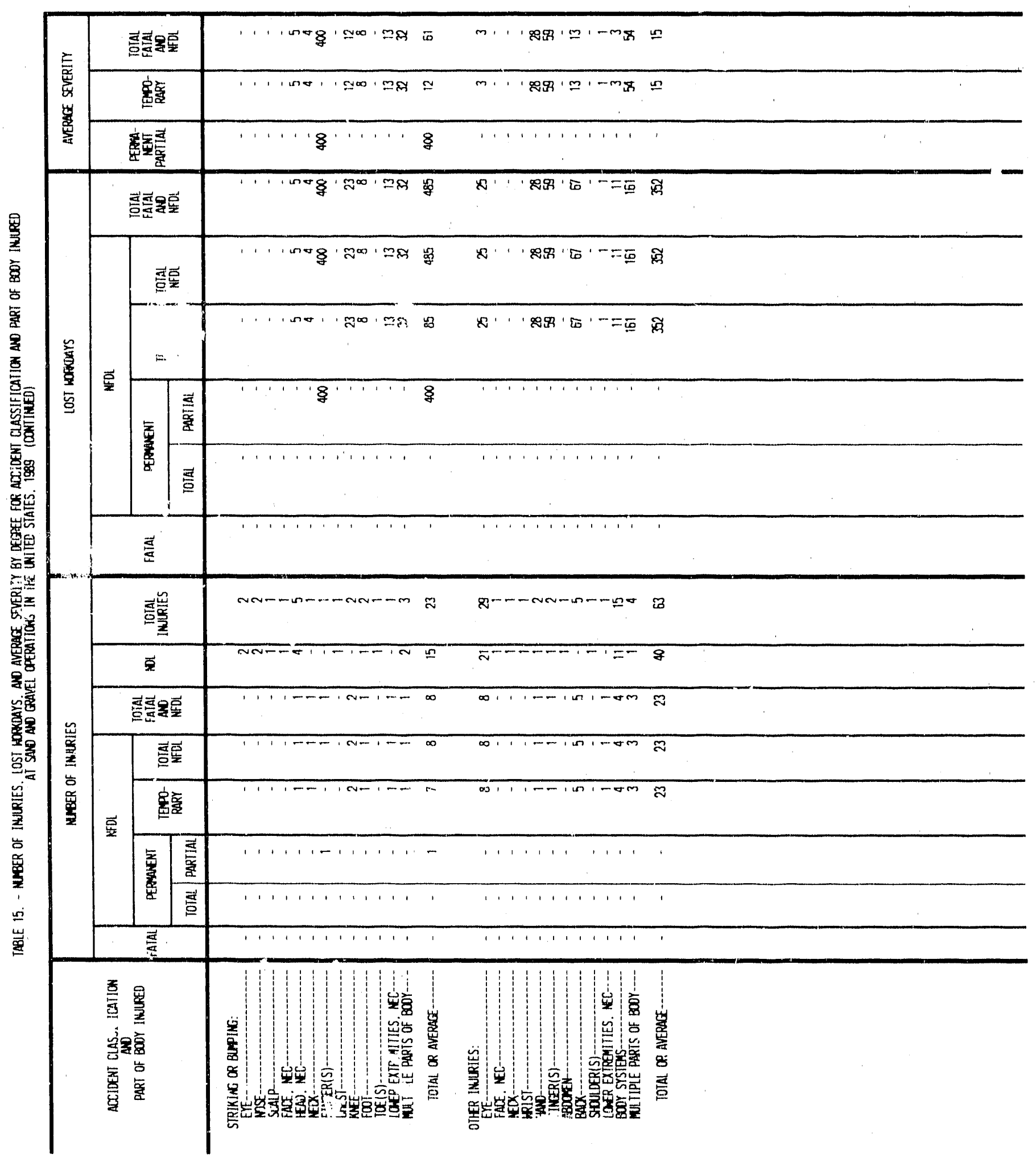




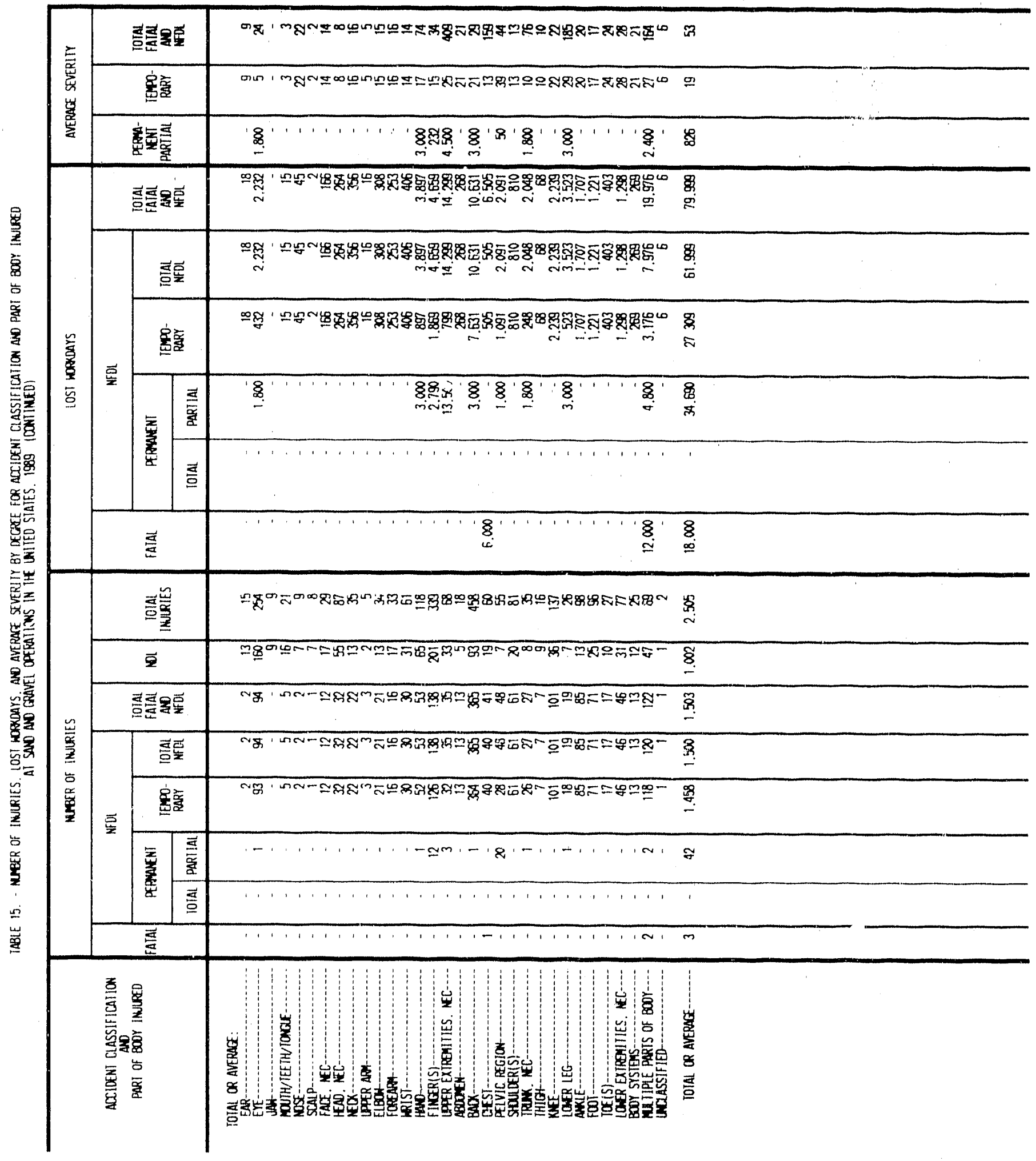




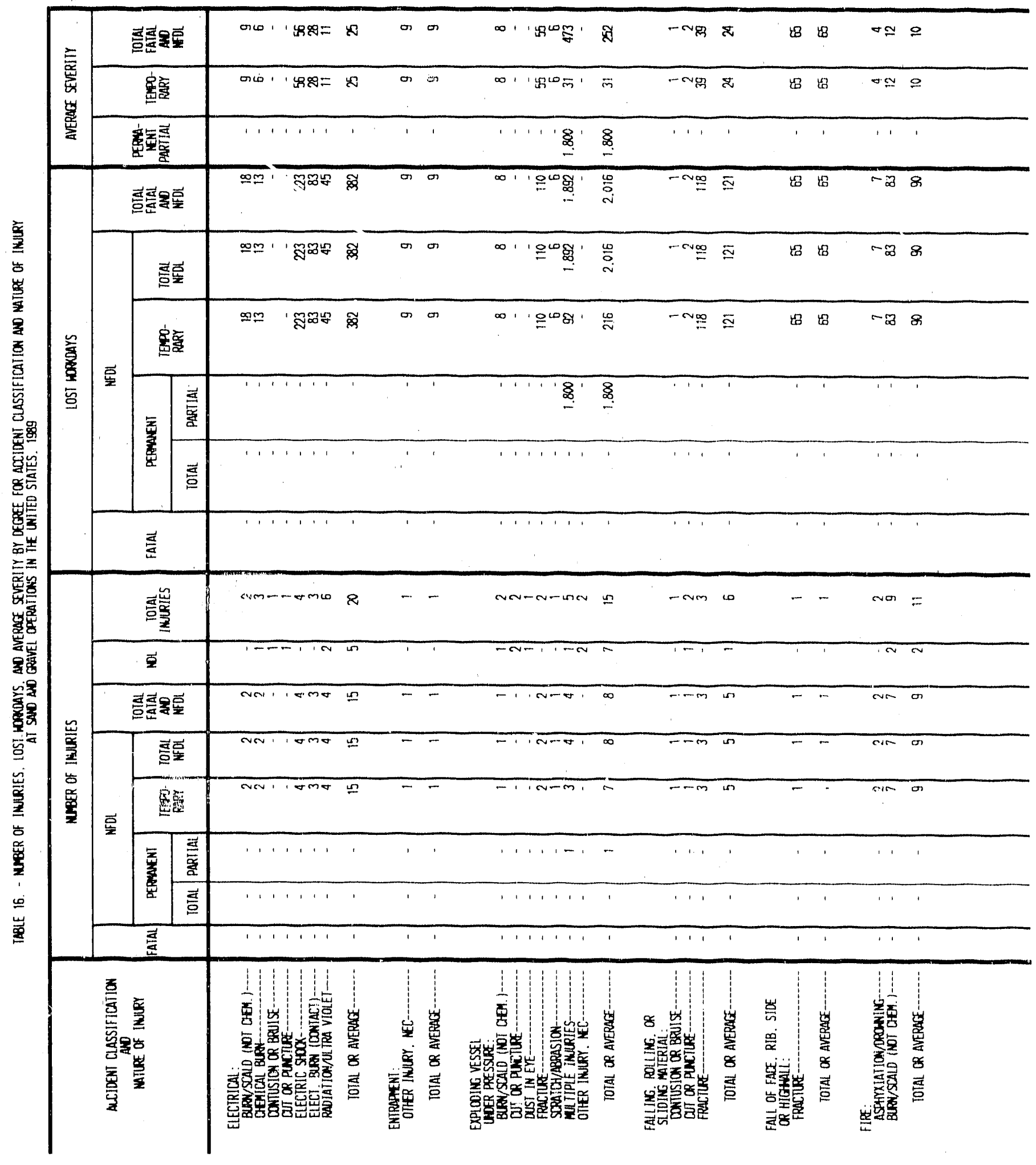




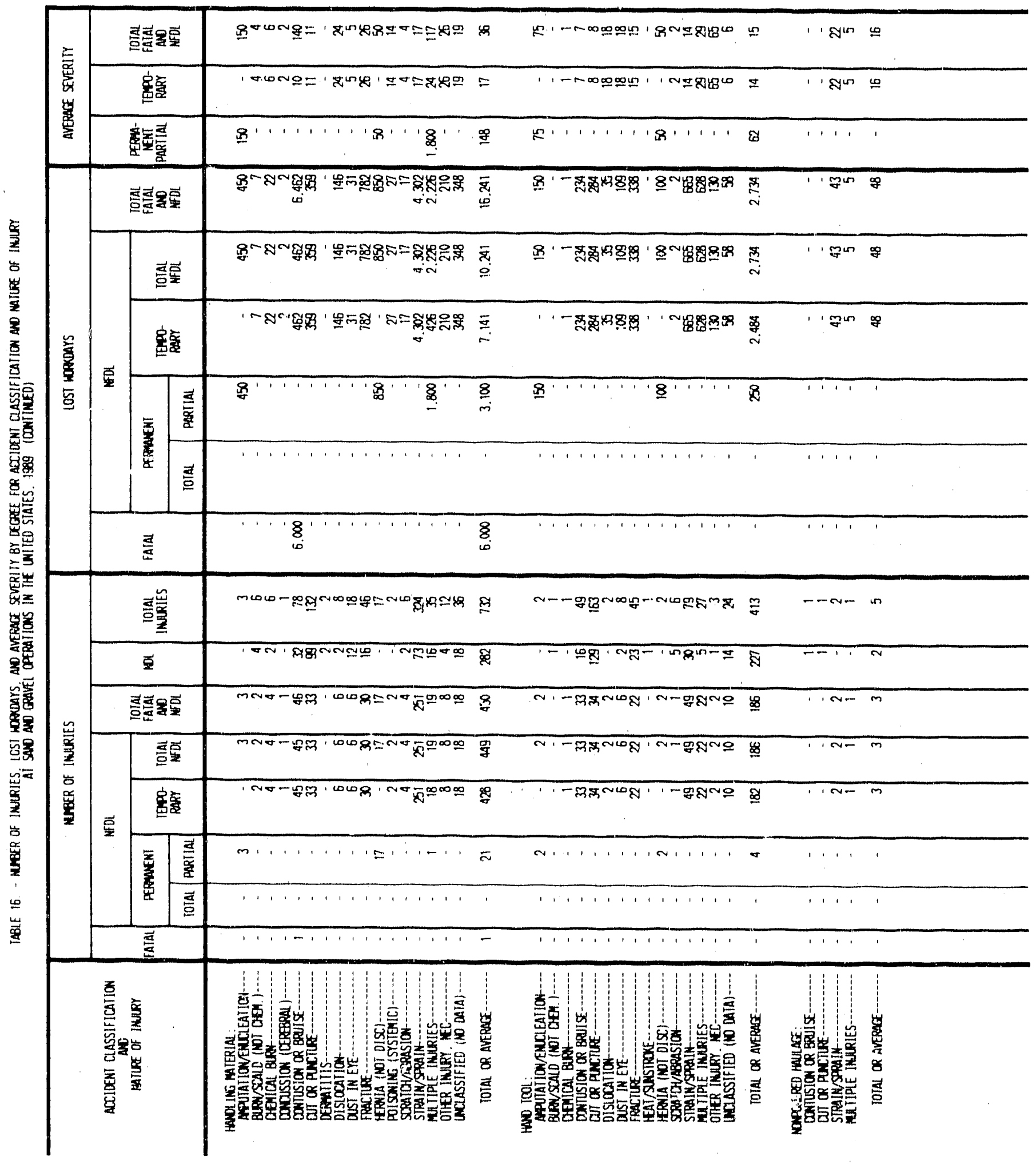




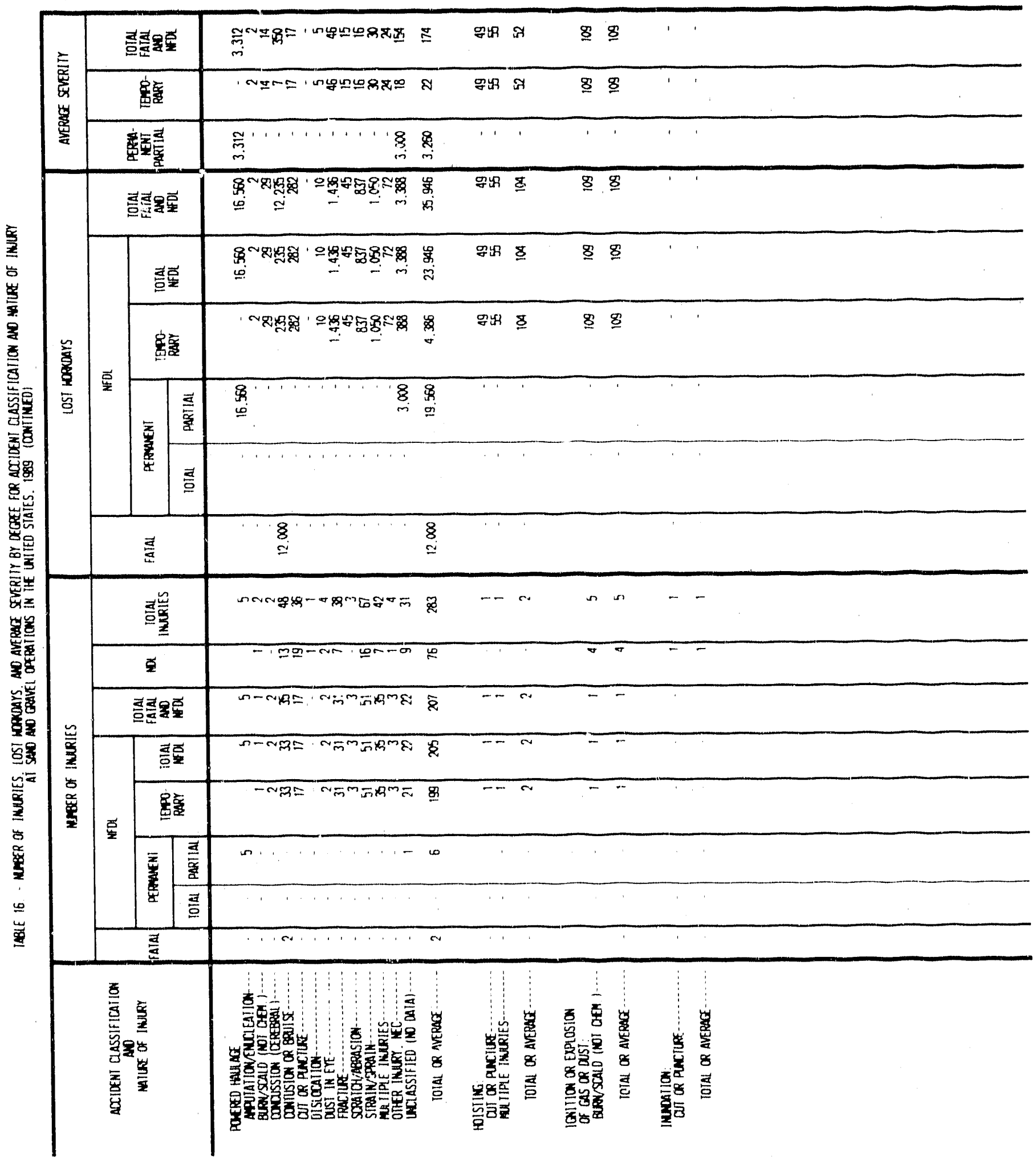




\begin{tabular}{|c|c|c|c|c|c|c|}
\hline \multirow{3}{*}{ 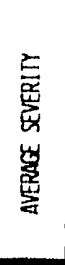 } & \multicolumn{2}{|c|}{ 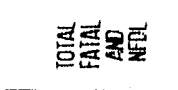 } & 雨ㅁ & 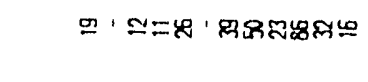 & $\infty$ & mong \pm \\
\hline & \multicolumn{2}{|c|}{ 密亳 } & 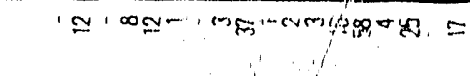 & 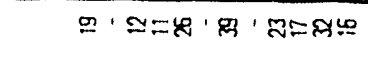 & $\mathscr{7}$ & mழ彑묘 I \\
\hline & \multicolumn{2}{|l|}{ 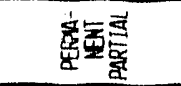 } & 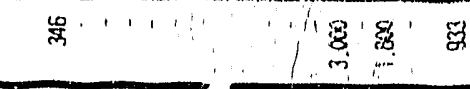 & 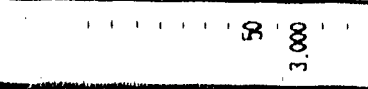 & $\stackrel{4}{-}$ & $\cdots$ \\
\hline & \multicolumn{2}{|c|}{ 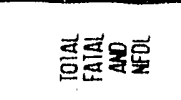 } & 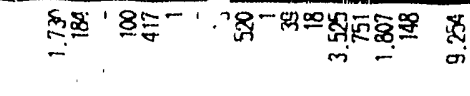 & 머 ' & $\stackrel{\bar{B}}{=}$ & 684 \\
\hline & \multirow{4}{*}{ 豆 } & 言垔 & 员各 ' & 머 ' & $\stackrel{\ddot{G}}{=}$ & ம马如 $\cong$ \\
\hline & & 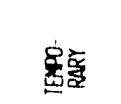 & 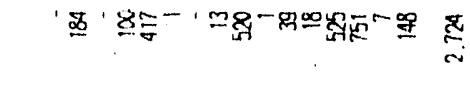 & 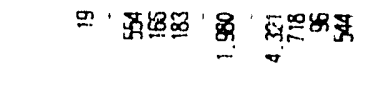 & $\begin{array}{c}\overline{5} \\
\infty \\
\infty \\
\infty\end{array}$ & ம৪द् $\cong$ \\
\hline & & $\begin{array}{l}\text { 롱 } \\
\text { 高 }\end{array}$ & 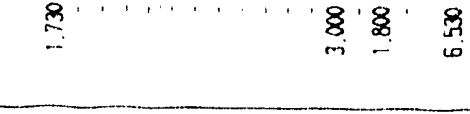 & ' $81 \underset{i}{8}$ & 兽 & \\
\hline & & $\underline{a}$ & . & & & \\
\hline & \multicolumn{2}{|l|}{$\underline{z}$} & & & & \\
\hline \multirow{8}{*}{ 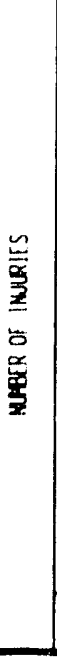 } & \multicolumn{2}{|c|}{ 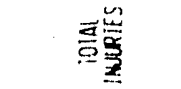 } & n & $-\cos \theta-3-x$ & 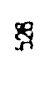 & $\operatorname{arng} f$ \\
\hline & \multicolumn{2}{|l|}{$\overline{\mathbf{g}}$} & 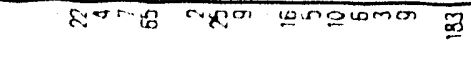 & 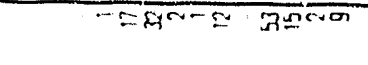 & 五 & -19 \\
\hline & \multicolumn{2}{|c|}{ 흠원요 } & 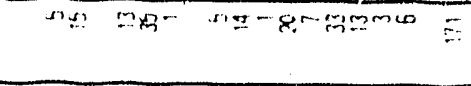 & f내 5 - & $\not$ & misg: g! \\
\hline & \multicolumn{2}{|c|}{$\overline{\underline{z}}$} & 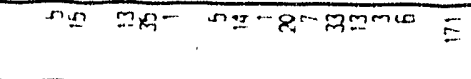 & S高 & 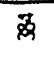 & ming. ge \\
\hline & \multicolumn{2}{|c|}{8} & 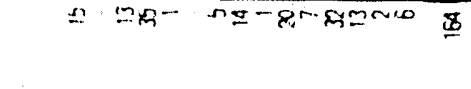 & 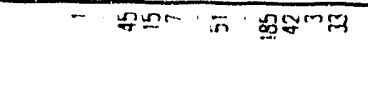 & 9 & $\operatorname{lng} 8$ \\
\hline & & \begin{tabular}{|l|}
$\underline{a}$ \\
a \\
\end{tabular} & $n$ & - & $r$ & \\
\hline & 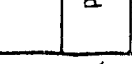 & 总 & & & & ' \\
\hline & \multicolumn{2}{|l|}{ 폴 } & & & & \\
\hline & \multicolumn{2}{|l|}{ 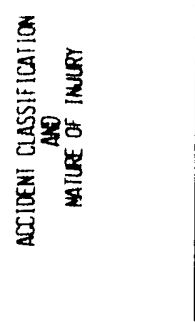 } & 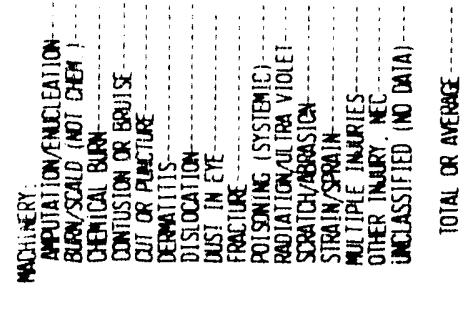 & 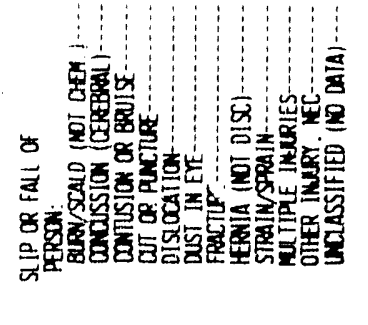 & 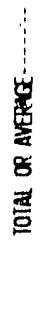 & 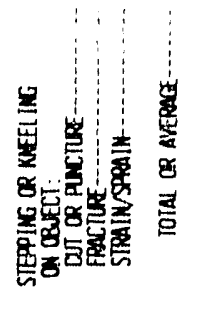 \\
\hline
\end{tabular}




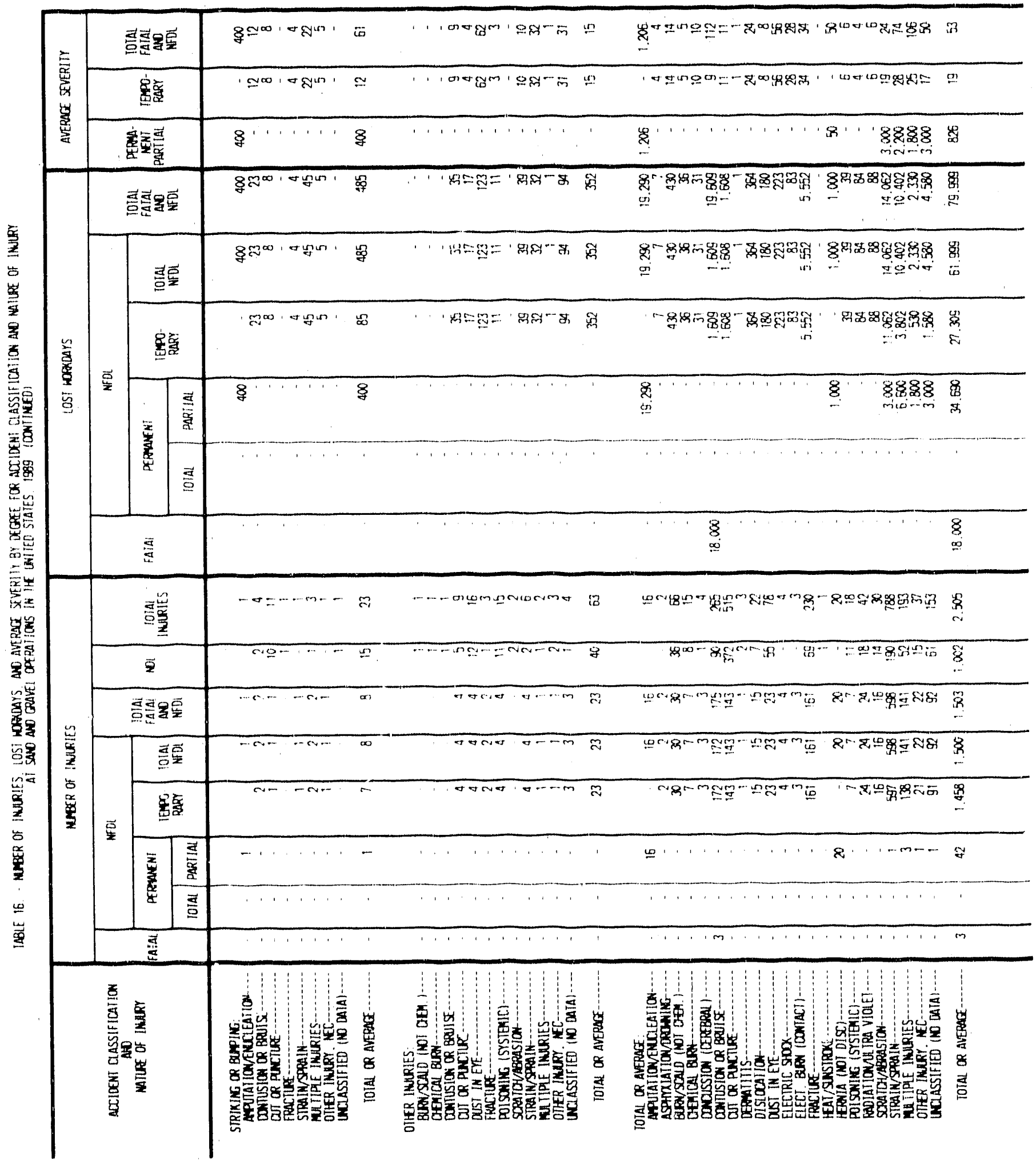




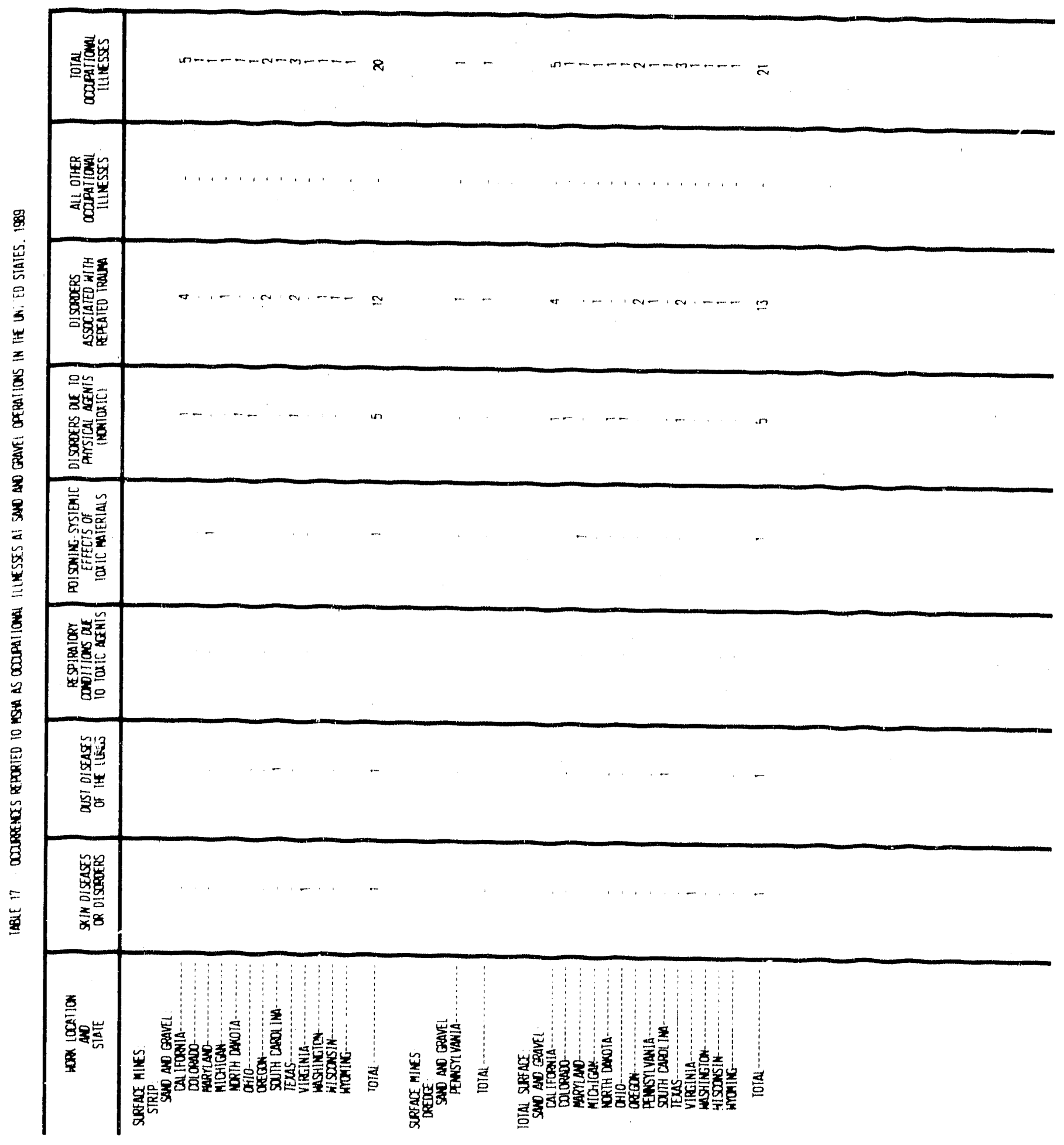




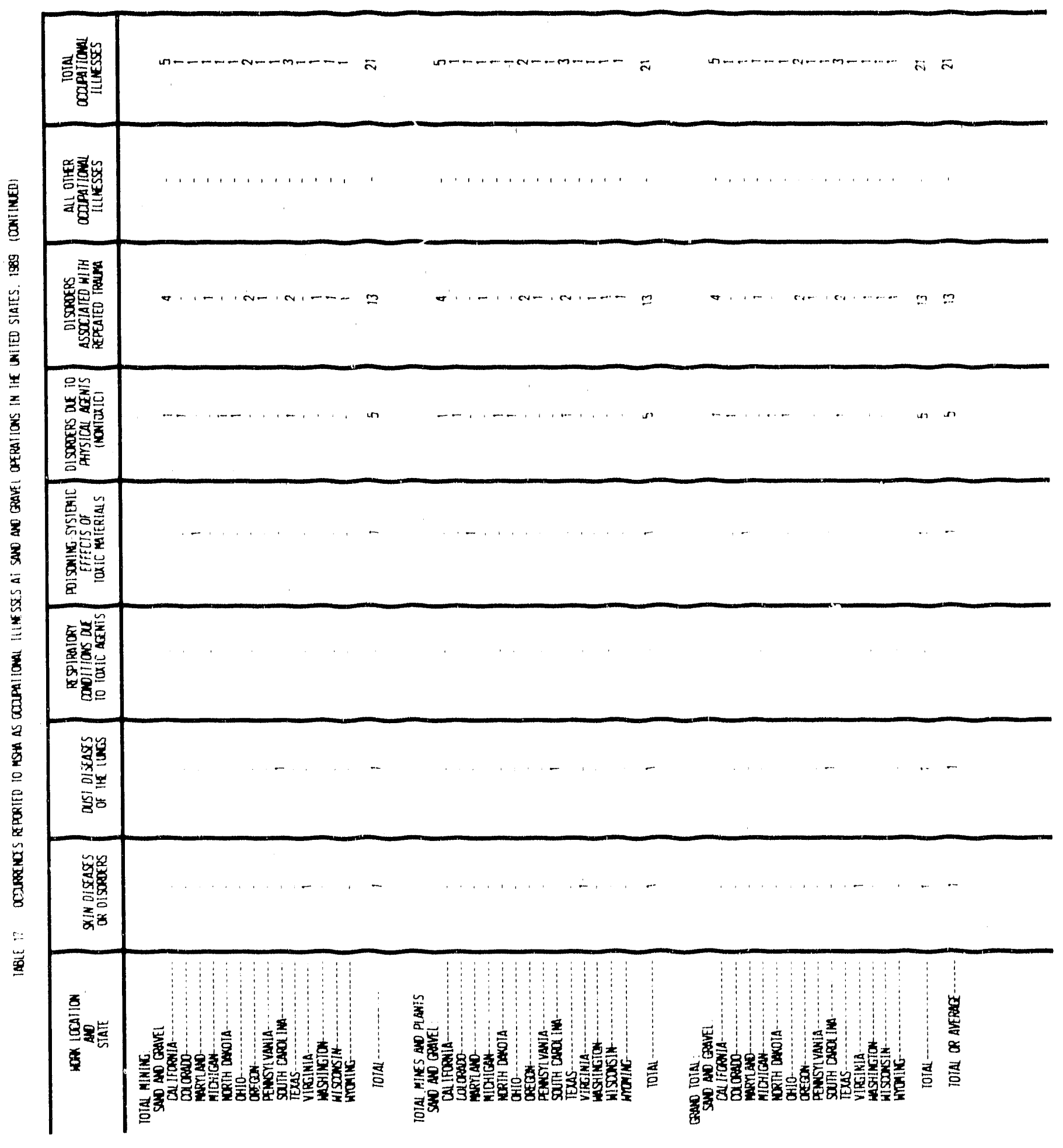




\begin{tabular}{|c|c|c|c|c|c|c|c|}
\hline 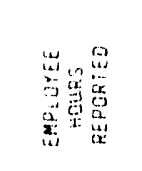 & 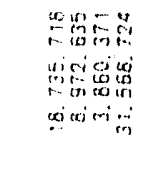 & 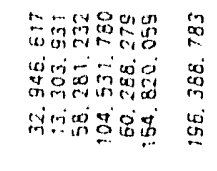 & 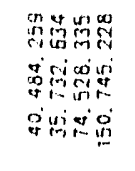 & 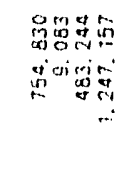 & 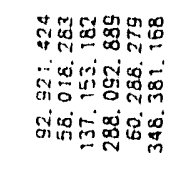 & 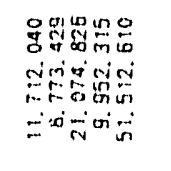 & 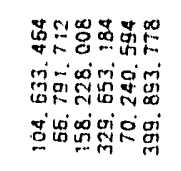 \\
\hline 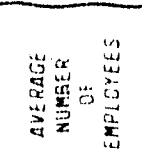 & 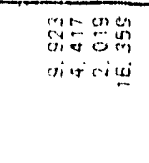 & 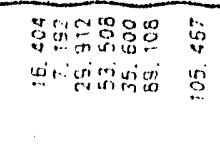 & 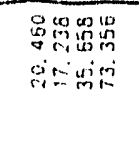 & 兽以融思 & 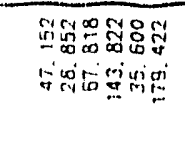 & 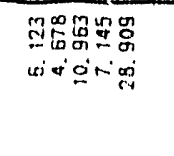 & 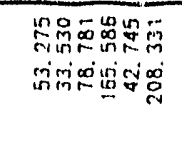 \\
\hline 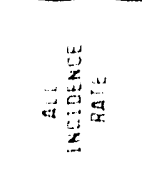 & 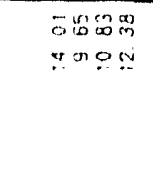 & 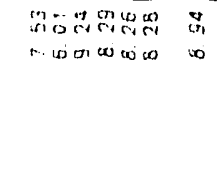 & 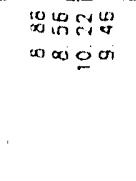 & 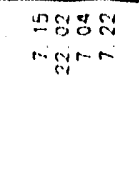 & 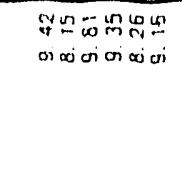 & 뜸송요 & 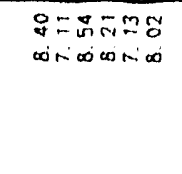 \\
\hline 离离 & 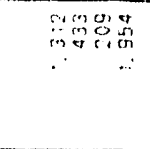 & 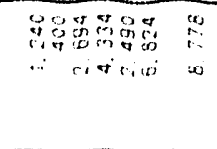 & 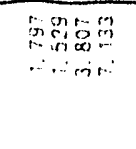 & $\therefore \cdots$ & 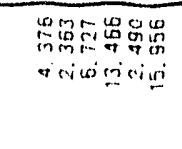 & $a \operatorname{mos}^{2}$ & 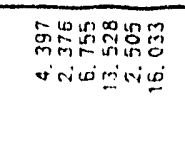 \\
\hline 类紧 & 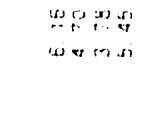 & 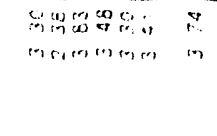 & 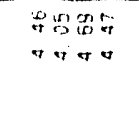 & 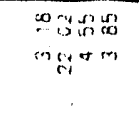 & 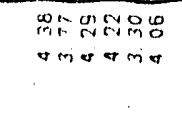 & 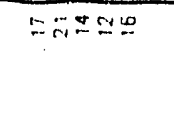 & 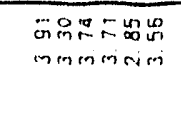 \\
\hline 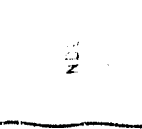 & 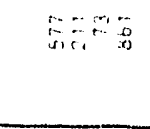 & 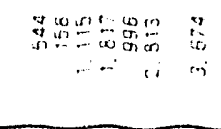 & 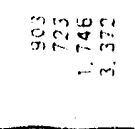 & $\because \cdots=8$ & 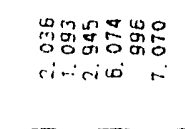 & ovingog & 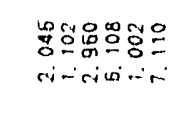 \\
\hline 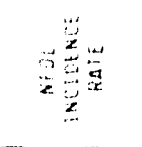 & 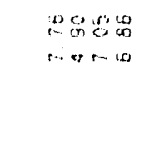 & 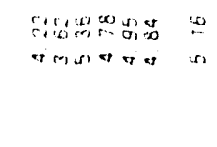 & 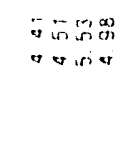 & 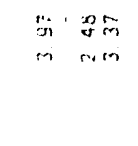 & 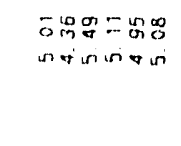 & $g g \div \infty \Phi$ & 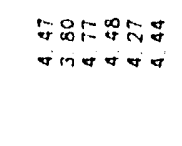 \\
\hline 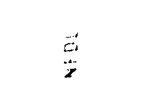 & 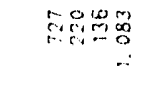 & 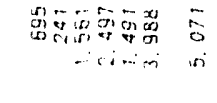 & 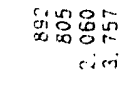 & $=0 \bar{r}$ & 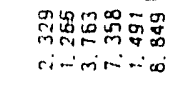 & $=\pi \operatorname{mon}_{m}$ & 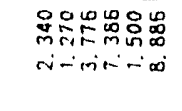 \\
\hline 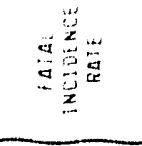 & y. & $0.8080 \%$ & 0ั0 & & 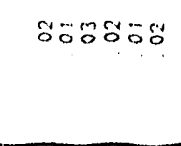 & & ชูㅇํㅇํㅇㅇㅠ \\
\hline $\begin{array}{l}\vec{a} \\
a \\
\end{array}$ & wasto & 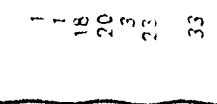 & $\cdots+r$ & ' & $=\sigma g r m$ & & $=\nabla \underline{m} m \bar{m}$ \\
\hline 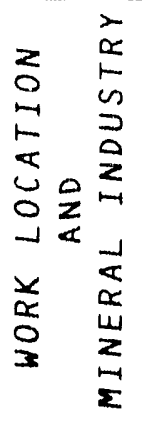 & 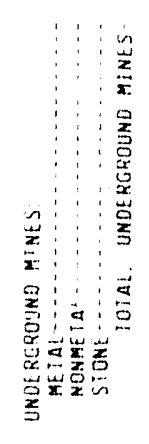 & 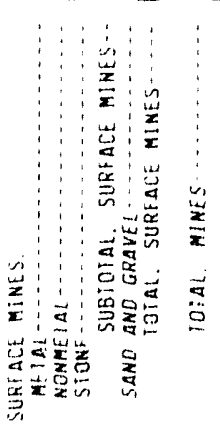 & 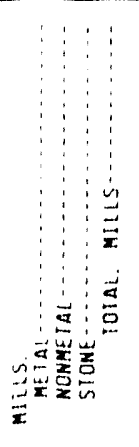 & 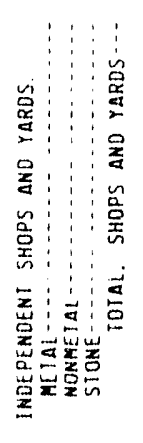 & 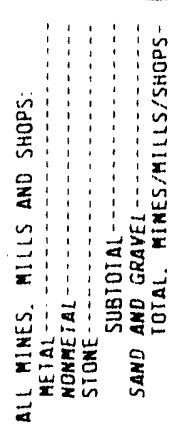 & 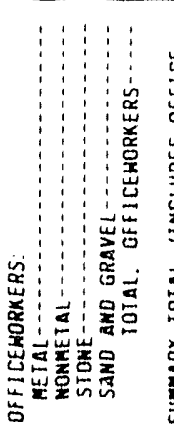 & 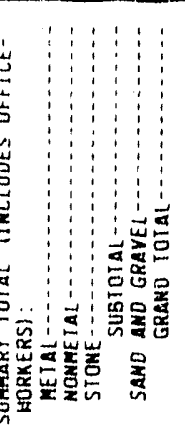 \\
\hline
\end{tabular}




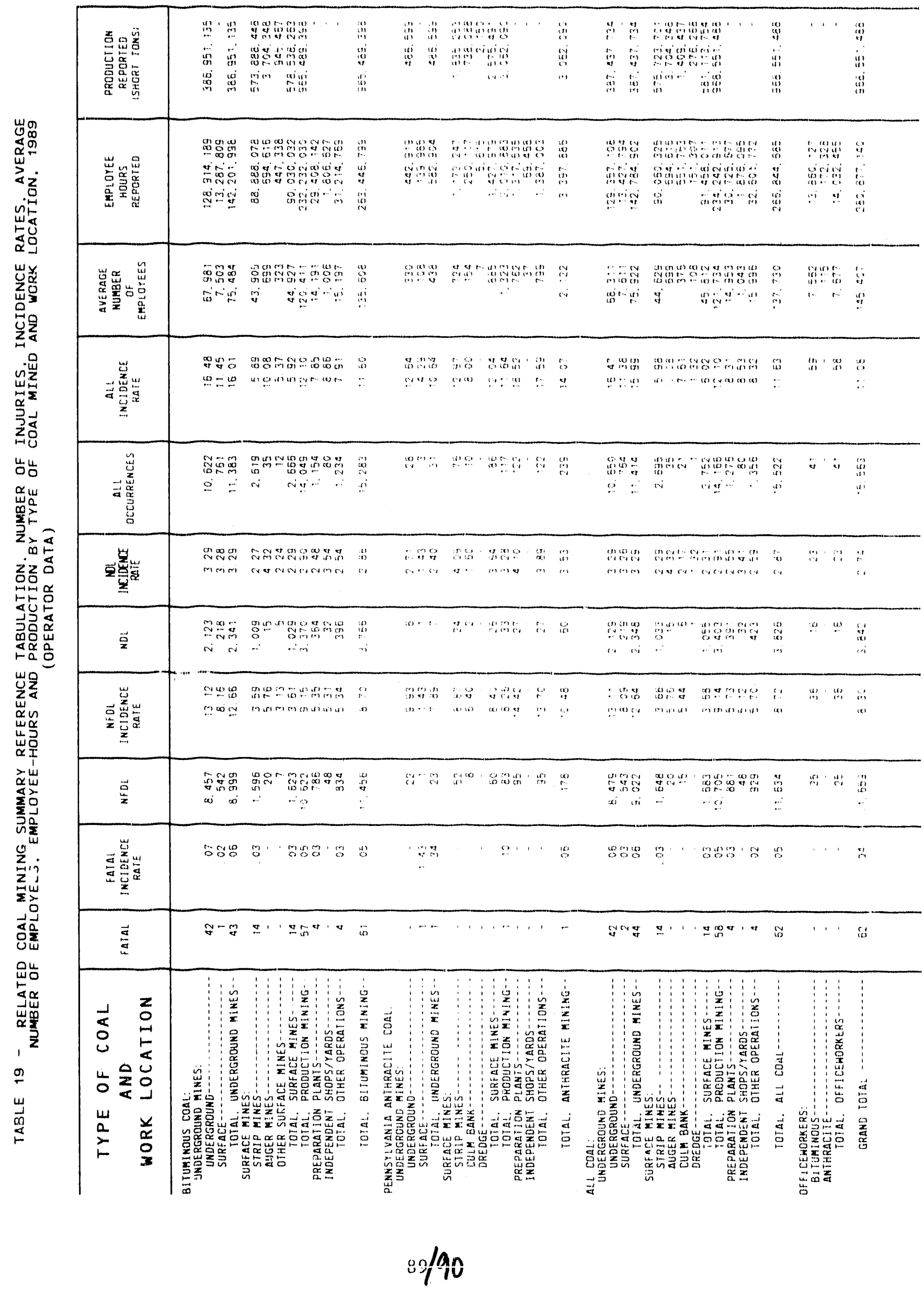


CONTRACTOR DATA 
The following reference gulde facilitates locating particular data elements contained in the annual statiatical tablea for contracters. The number appearing in each cell indicates the table number in which that particular element may be found. All elementa listed under the stub headinga appear as vertical headings in the tables. Elementa 1iated under column headings appear as horfzontal headings. The only exception to this is table 1. In which the headings have been reversed.

To use this guide, read down or across the elements listed in both the stub and column headings to determine which combinations are of interest. For example, 1ost workdays by work location appear in six different tables, numbers $5,7,9,12,13$, and 14. However, only one of these, table 9. provides a further division by nature of injury as well. The occupation of the injured is found in table 13 and, as seen in the column headings, provides the number of injuries, lost workdays, and average severity.

Al though injuries and ilinesses occurring to contractor employees are identifiable as to state in which they occurred or commodity mined, matching exposure hours are not available. Incidence ratea for auch employees are possible only at the national level for the various subunits and only for the coal industry as a whole and the noncoal industry as a whole. Consequently, certain tables have been entirely eliminated from the contractor portion of this report. The remaining tables are numbered to correspond with the comparable table in the operator portion of this report. 


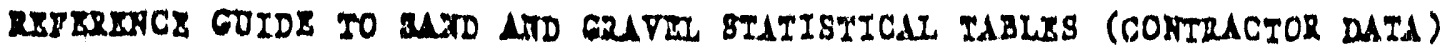

\begin{tabular}{|c|c|c|c|c|c|c|c|c|c|}
\hline 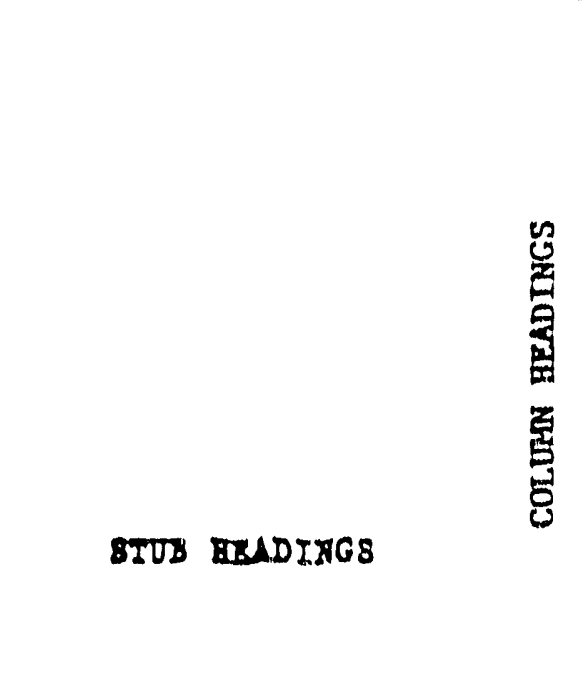 & 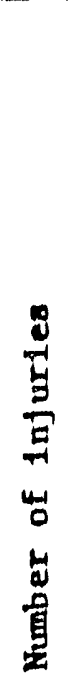 & 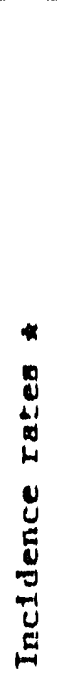 & 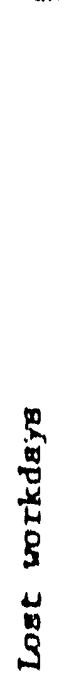 & 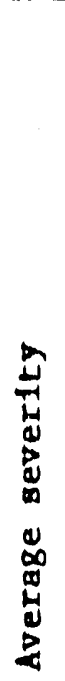 & 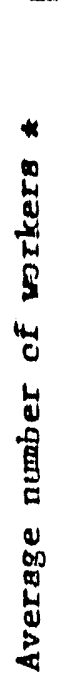 & 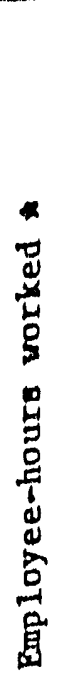 & 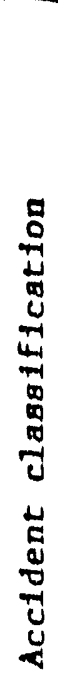 & 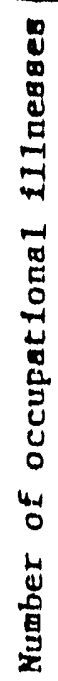 & 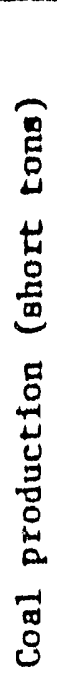 \\
\hline $\begin{array}{l}\text { Prior joar data } \\
\text { rith urd vitbout office } 11\end{array}$ & 1 & & & 1 & & & & & \\
\hline \multirow{10}{*}{ Work locatios } & 5 & & 5 & 5 & & & & & \\
\hline & 7 & & 7 & 7 & & & & & \\
\hline & 9 & & 9 & 9 & & & & & \\
\hline & 11 & & & & & & 11 & & \\
\hline & & & 12 & & & & 12 & & \\
\hline & 13 & & 13 & 13 & & & & & \\
\hline & 14 & & 14 & 14 & & & & & \\
\hline & & & & & & & & 17 & \\
\hline & 18 & 18 & & & 18 & 18 & & & \\
\hline & 19 & 19 & & & 19 & 19 & & & 19 \\
\hline \multirow{4}{*}{ state } & 5 & & 5 & 5 & & & & & \\
\hline & 11 & & & & & & 1.1 & & \\
\hline & & & 12 & & & & 12 & & \\
\hline & & & & & & & & 17 & \\
\hline \multirow{3}{*}{ Part of body iojured } & 7 & & 7 & 7 & & & & & \\
\hline & 10 & & 10 & 10 & & & & & \\
\hline & 15 & & 15 & 15 & & & & & \\
\hline \multirow{3}{*}{ Matura of injury } & 9 & & 9 & 9 & & & & & \\
\hline & 10 & & 10 & 10 & & & & & \\
\hline & 16 & & 16 & 16 & & & & & \\
\hline $\begin{array}{l}\text { Occupation at tive of } \\
\text { ininey }\end{array}$ & 13 & & 13 & 13 & & & & & \\
\hline \multirow{3}{*}{ Accidant clasaification } & 14 & & 14 & 14 & & & & & \\
\hline & 15 & & 15 & 15 & & & & & \\
\hline & 16 & & 16 & 16 & & & & & \\
\hline \multirow{3}{*}{$\begin{array}{l}\text { Kineral-iaduetey } \\
\text { Joe of conl }\end{array}$} & & & & & & & & 17 & \\
\hline & 18 & 18 & & & 18 & 18 & & & \\
\hline & 19 & 19 & & & 19 & 19 & & & 19 \\
\hline
\end{tabular}

$1 /$ Tor table 1 ooly, the headiags upe been reveraed to airplify this soide; that is, the dats olements indicatsd in the coluzer beadings for this table actalily apper as rertical hadings.

- Contractor data appearizg in table 13 for the coluan beadizgs deated ofth an astariat ( $\star$ ) is thi guide are obtainable only for the noncoal. Lodustas ia a wola sad do zot raprasent a particular gizeral industry. 


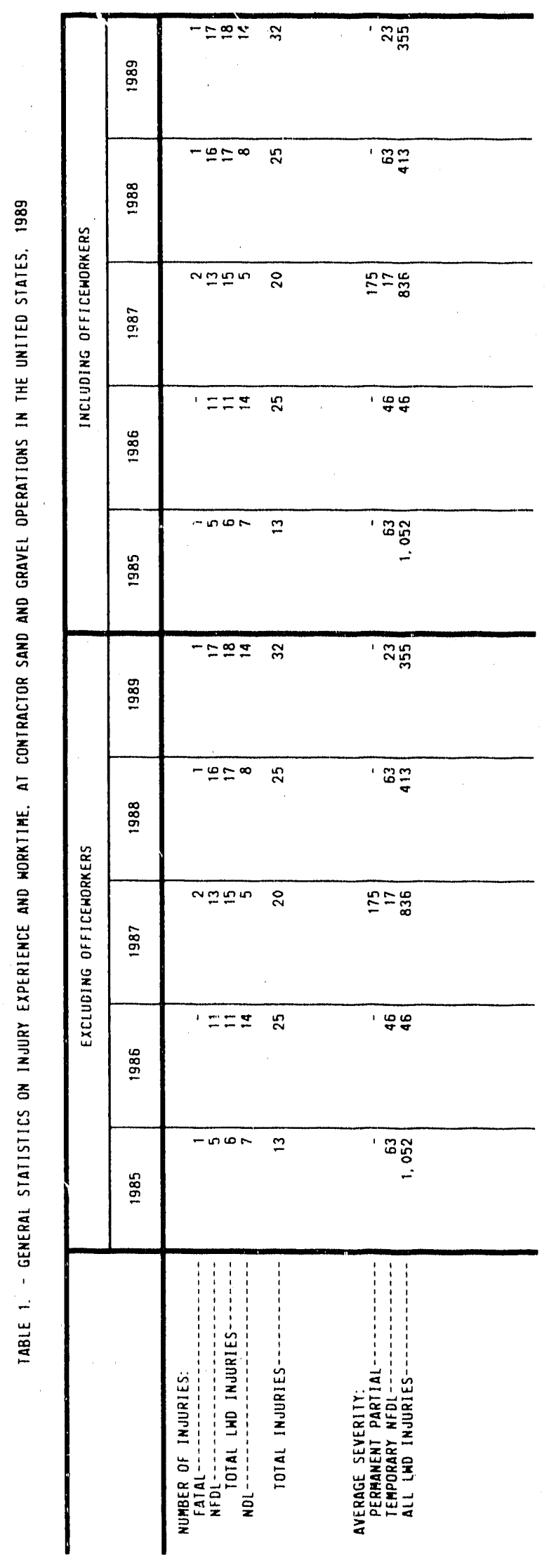




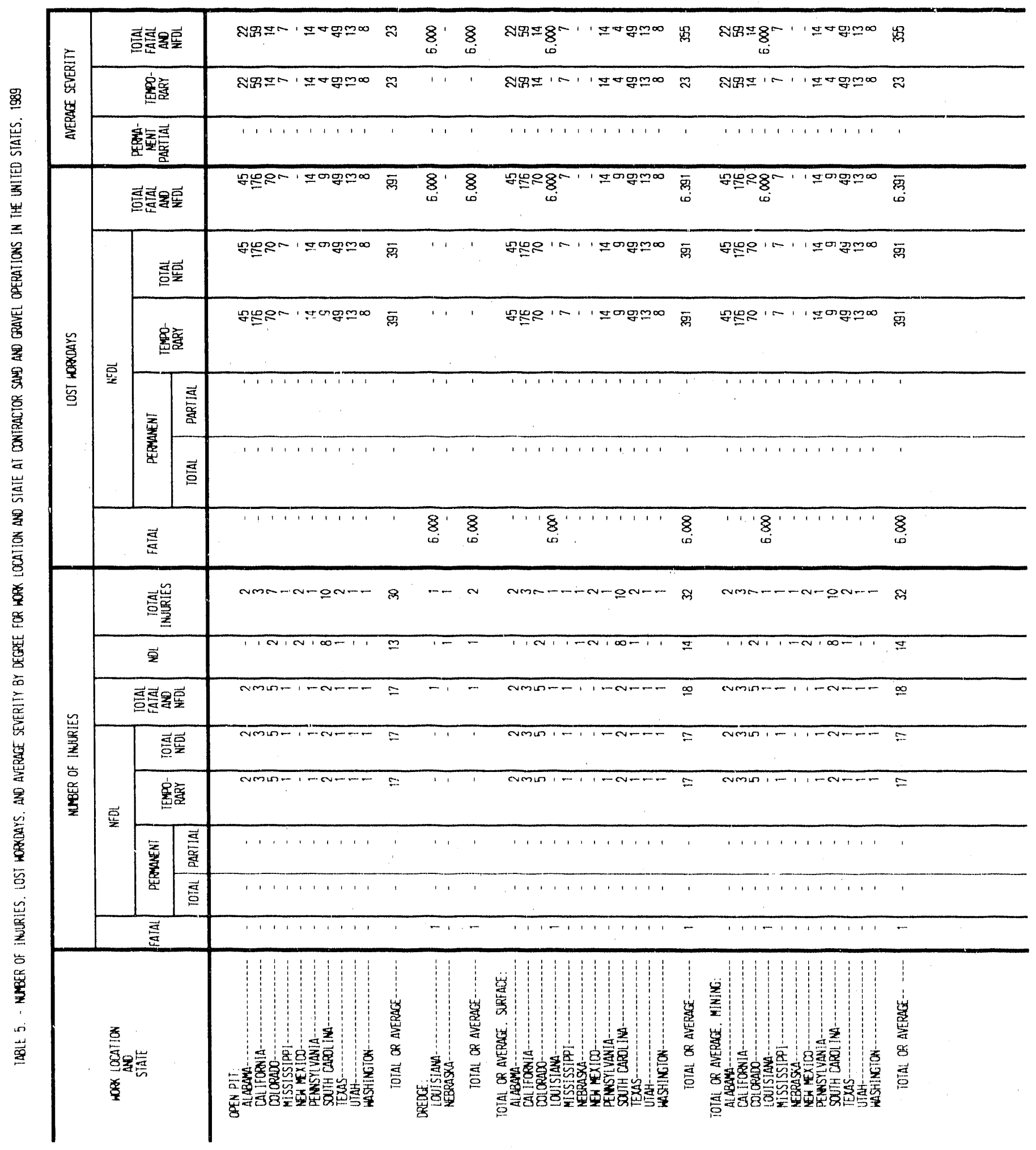




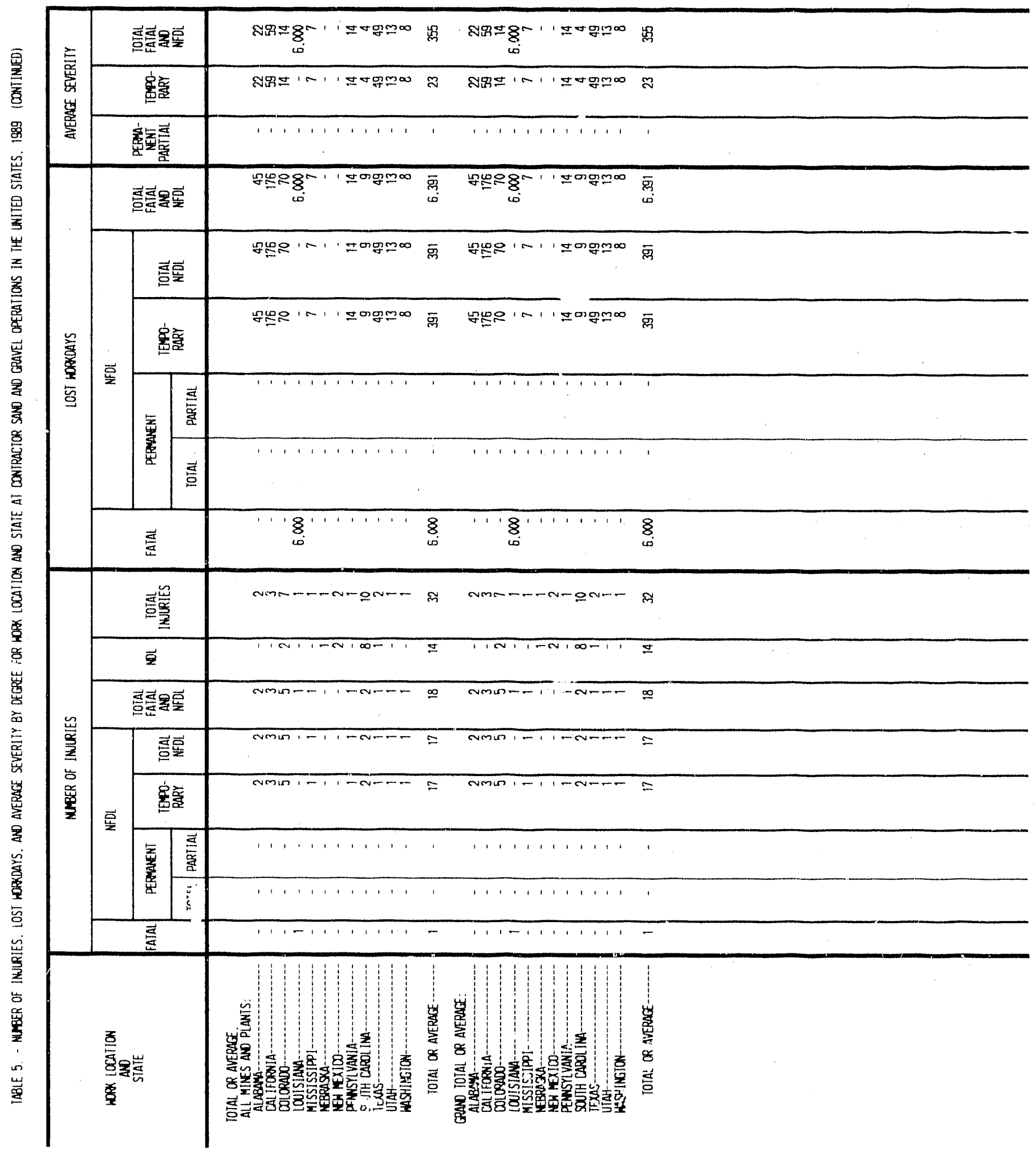




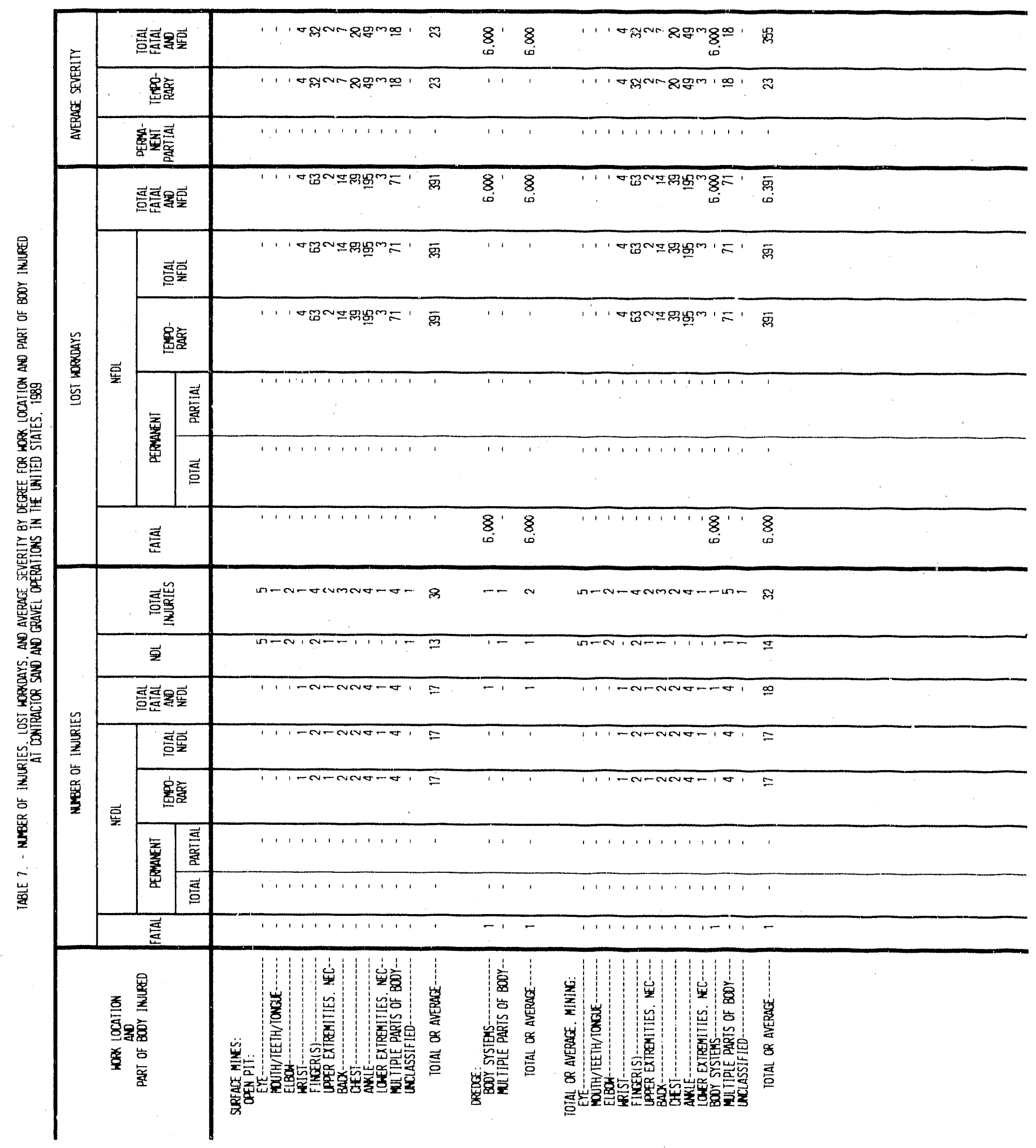




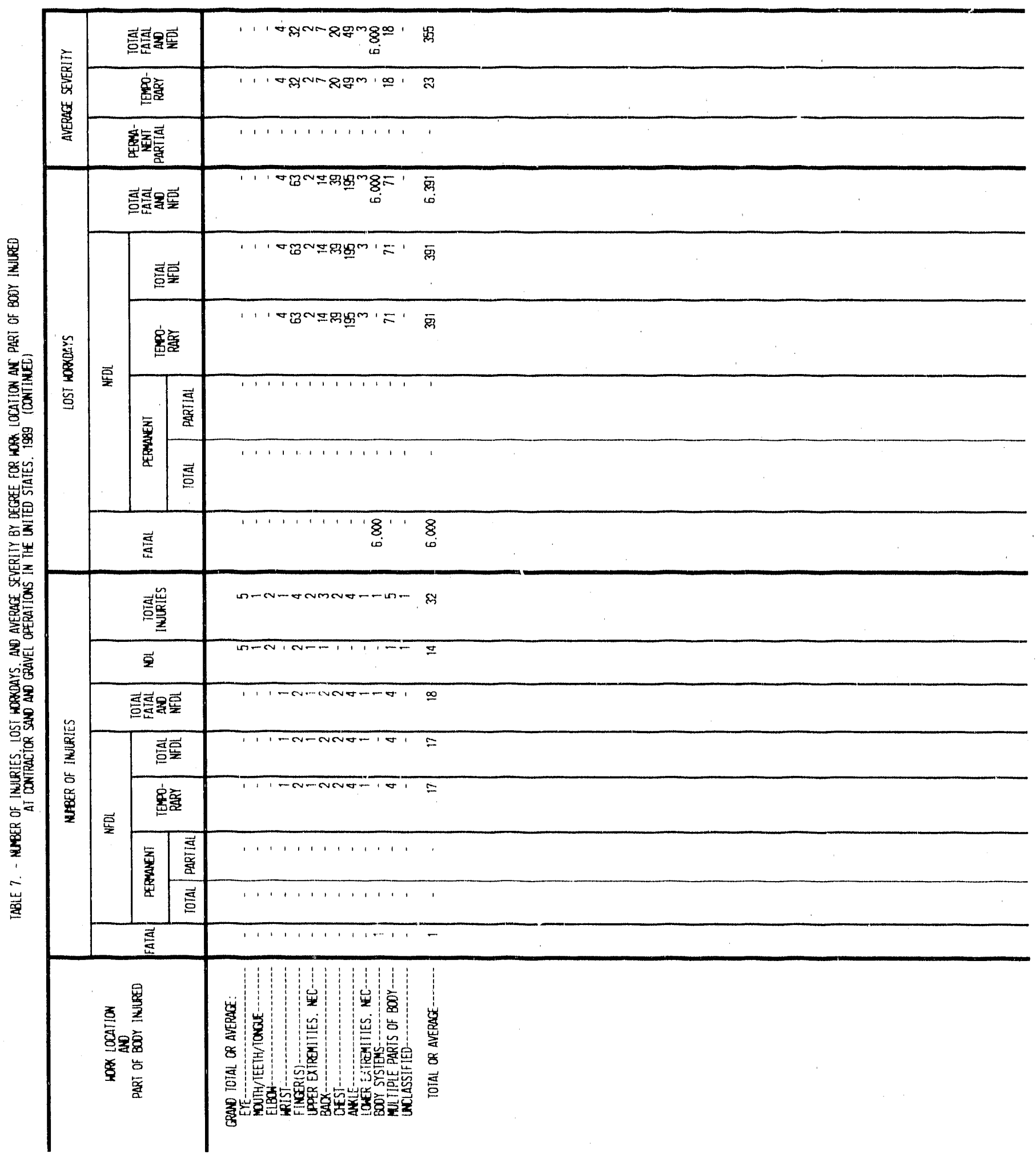




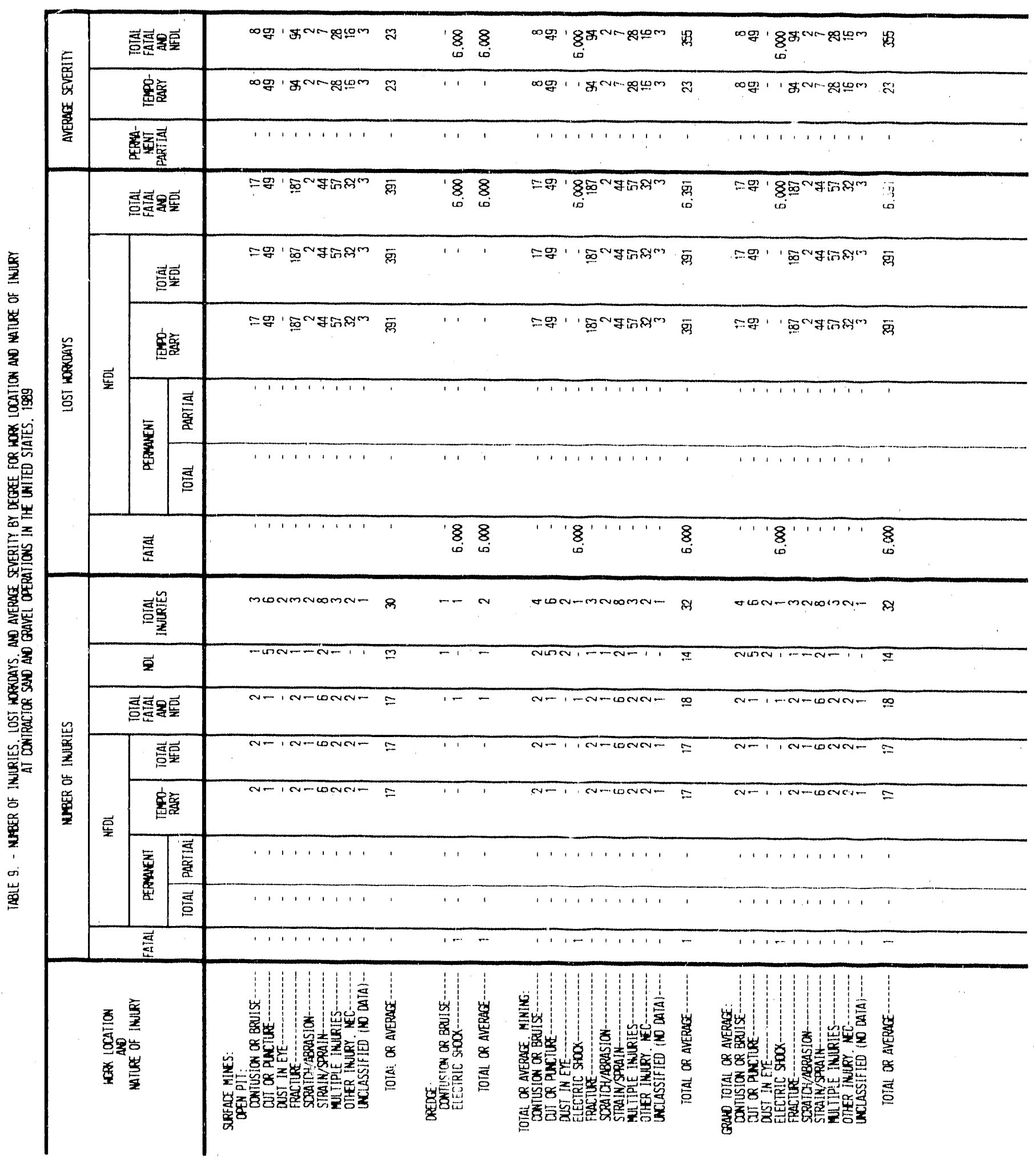




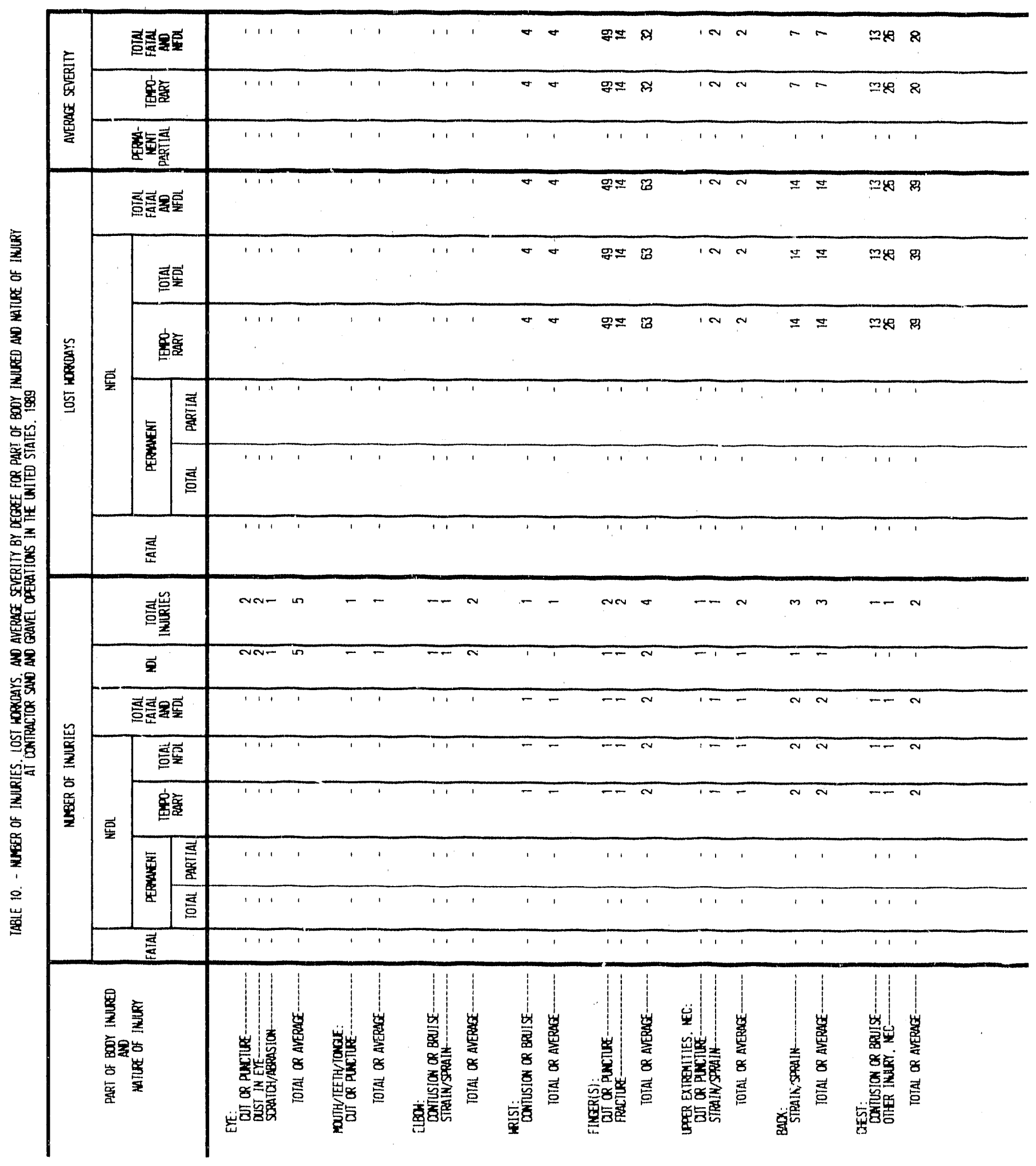




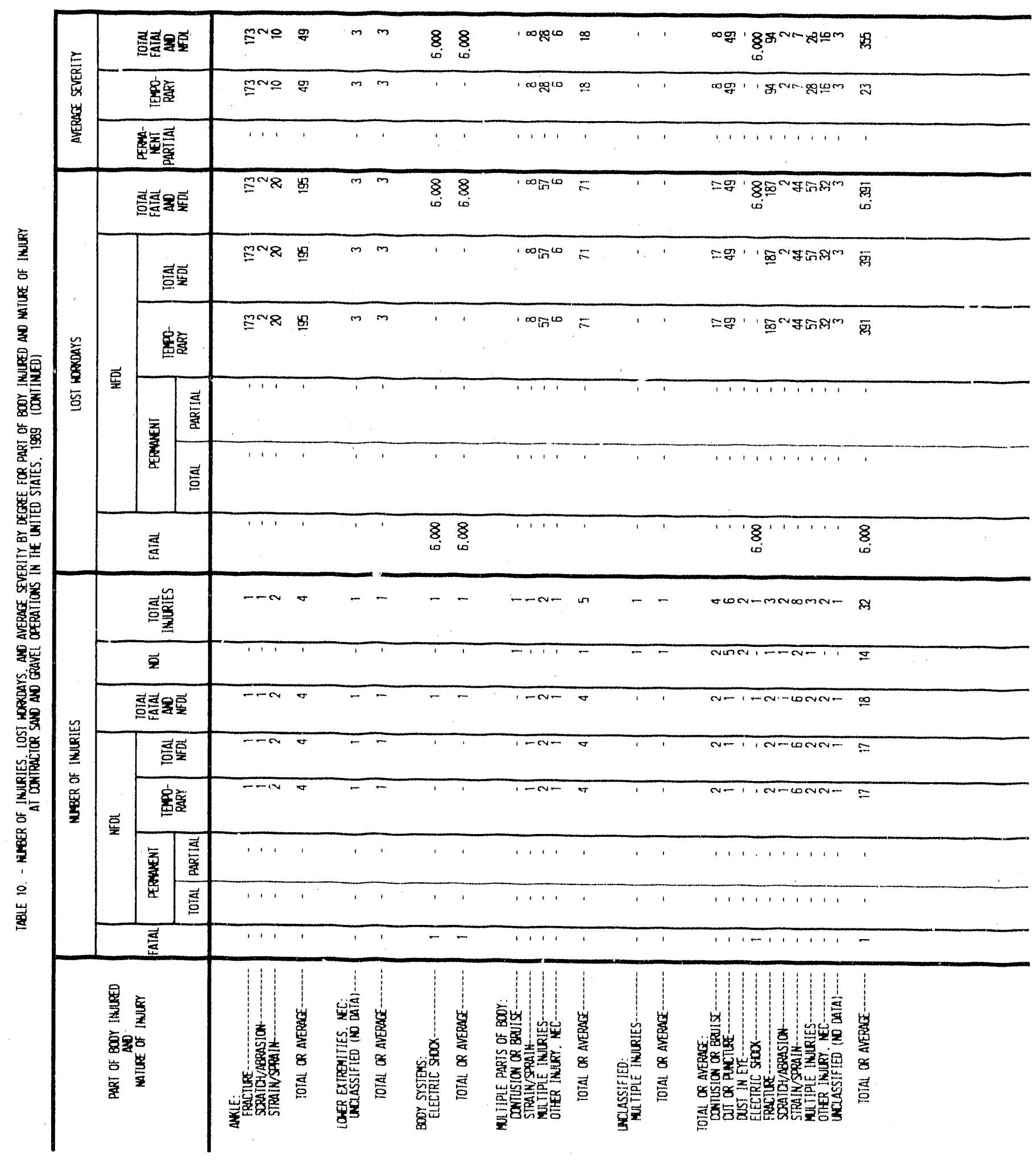




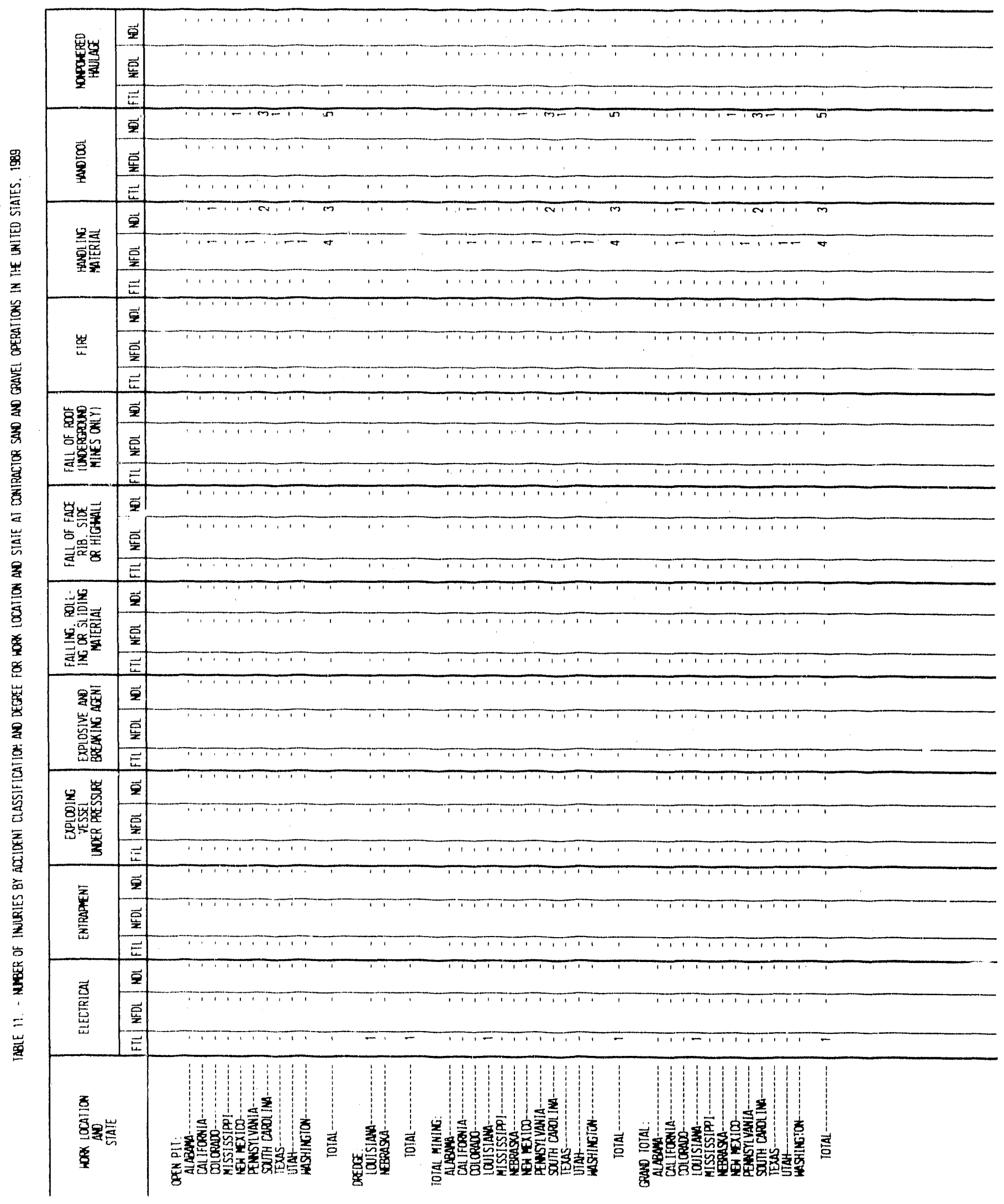




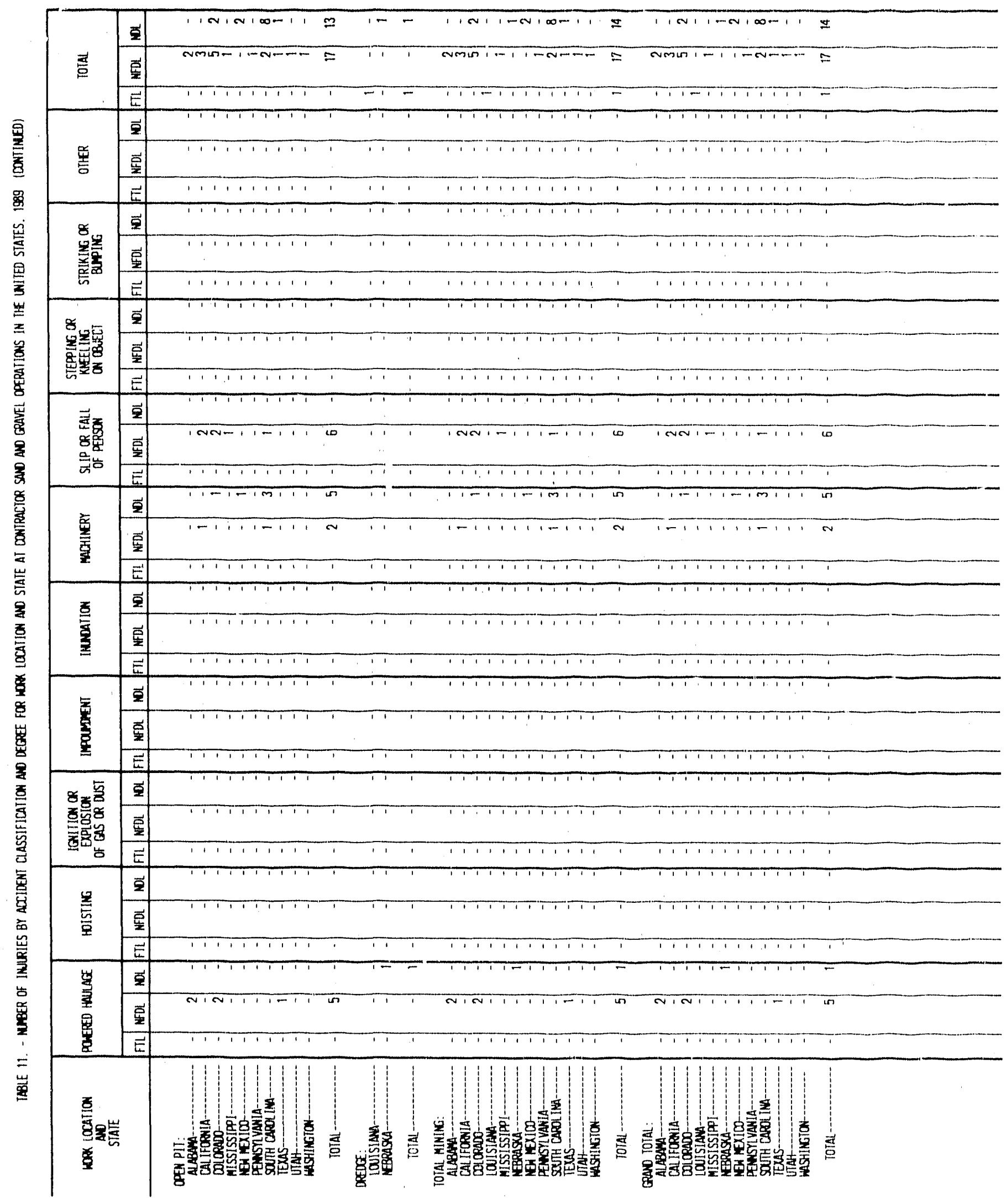




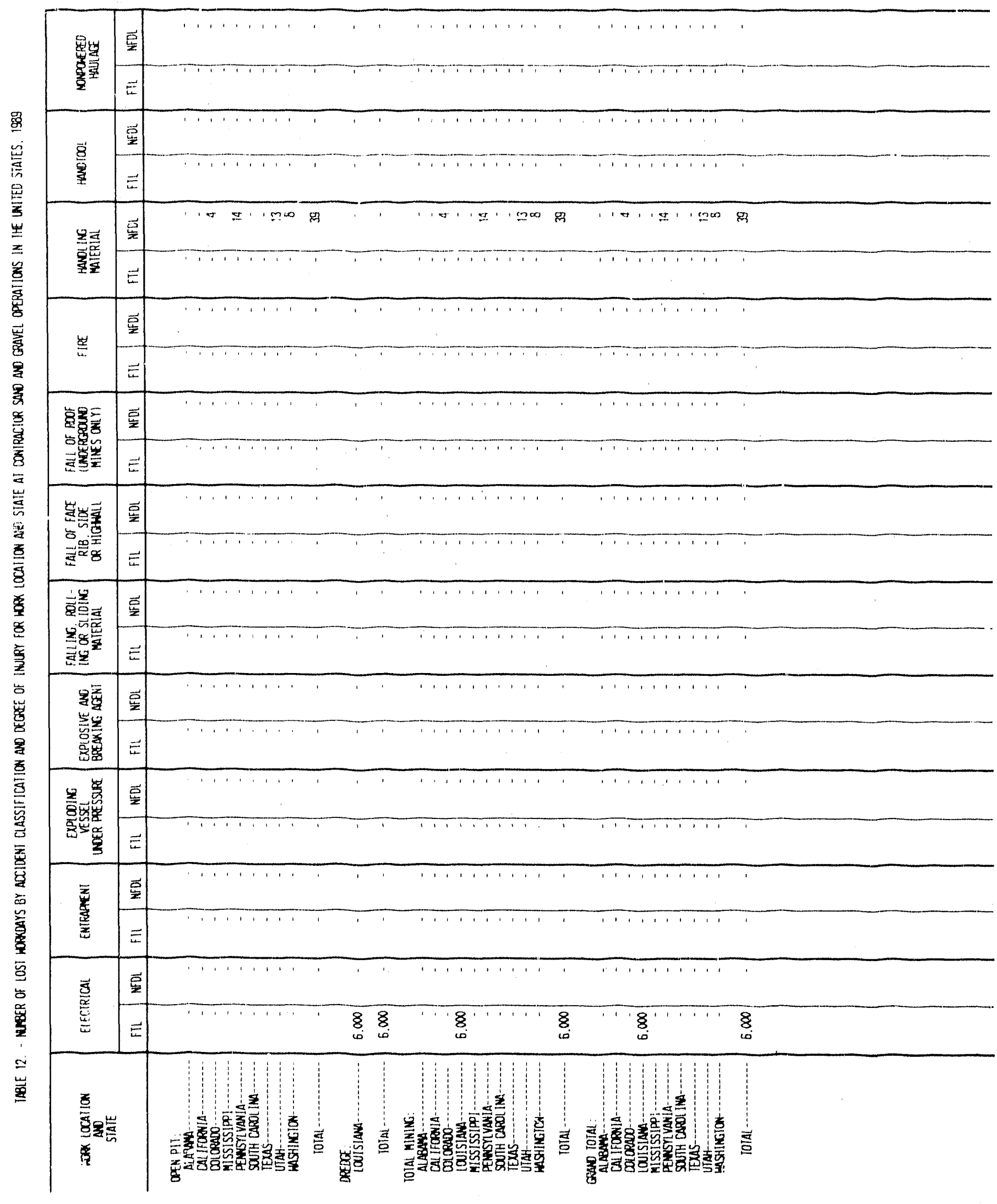




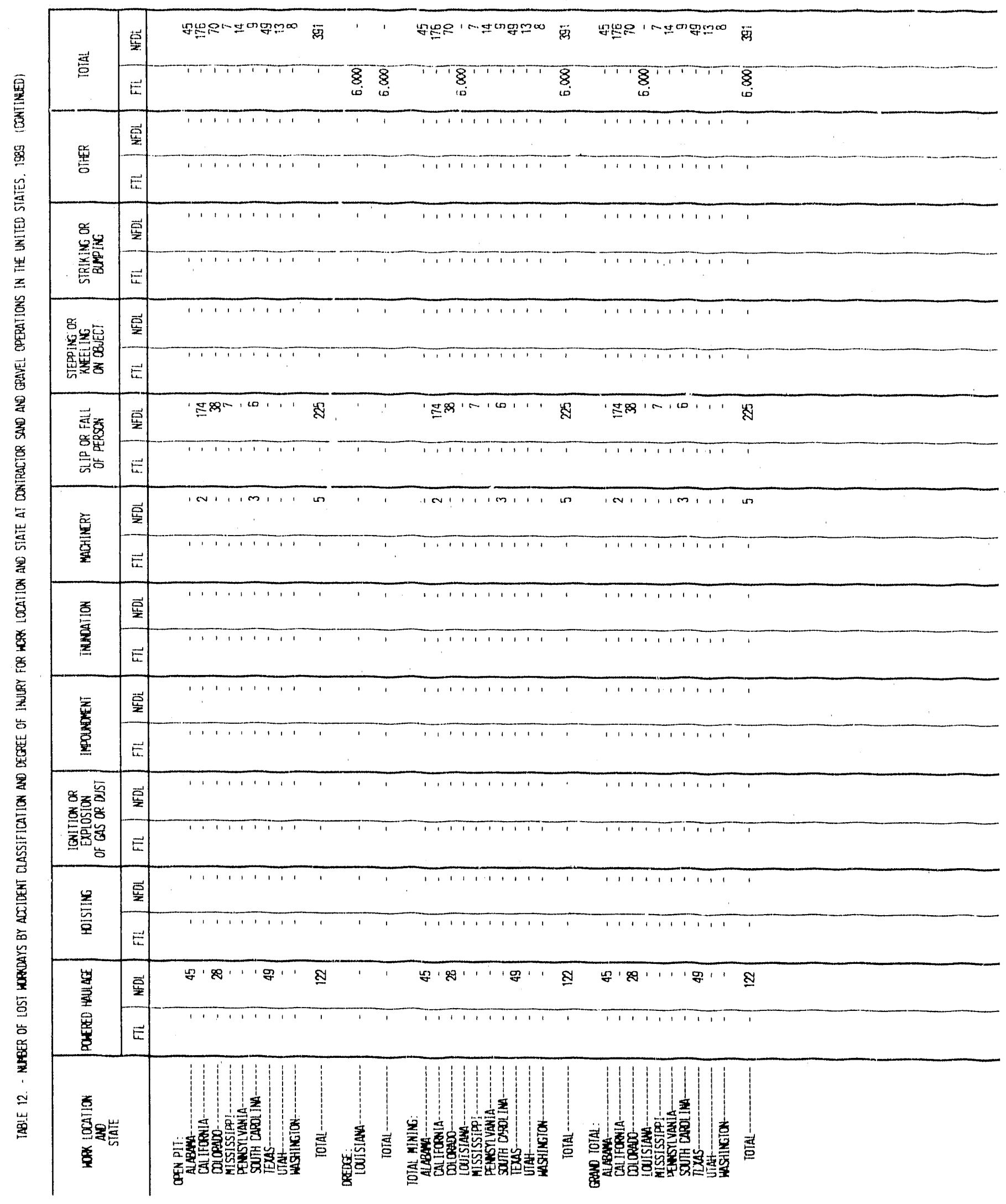




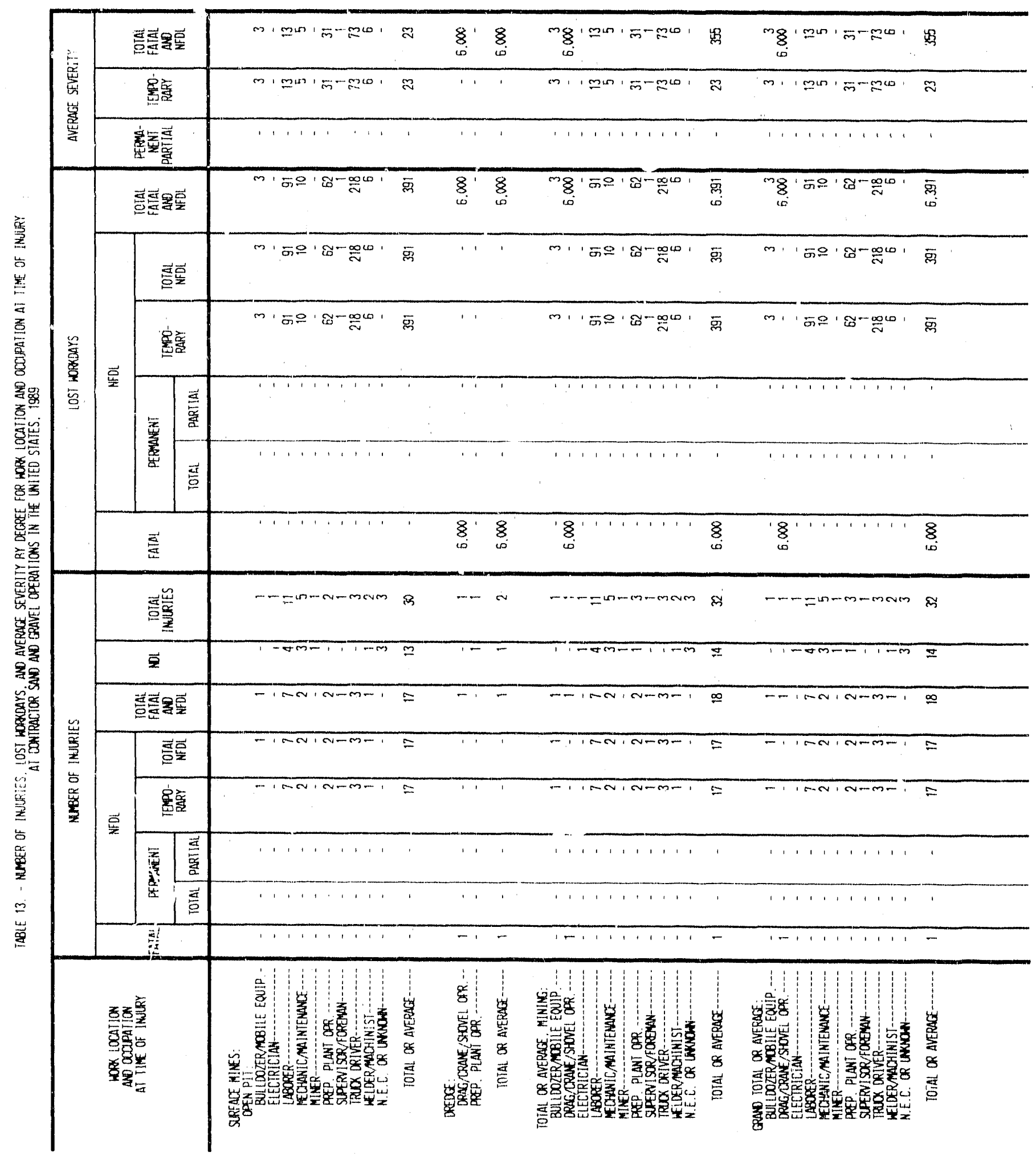




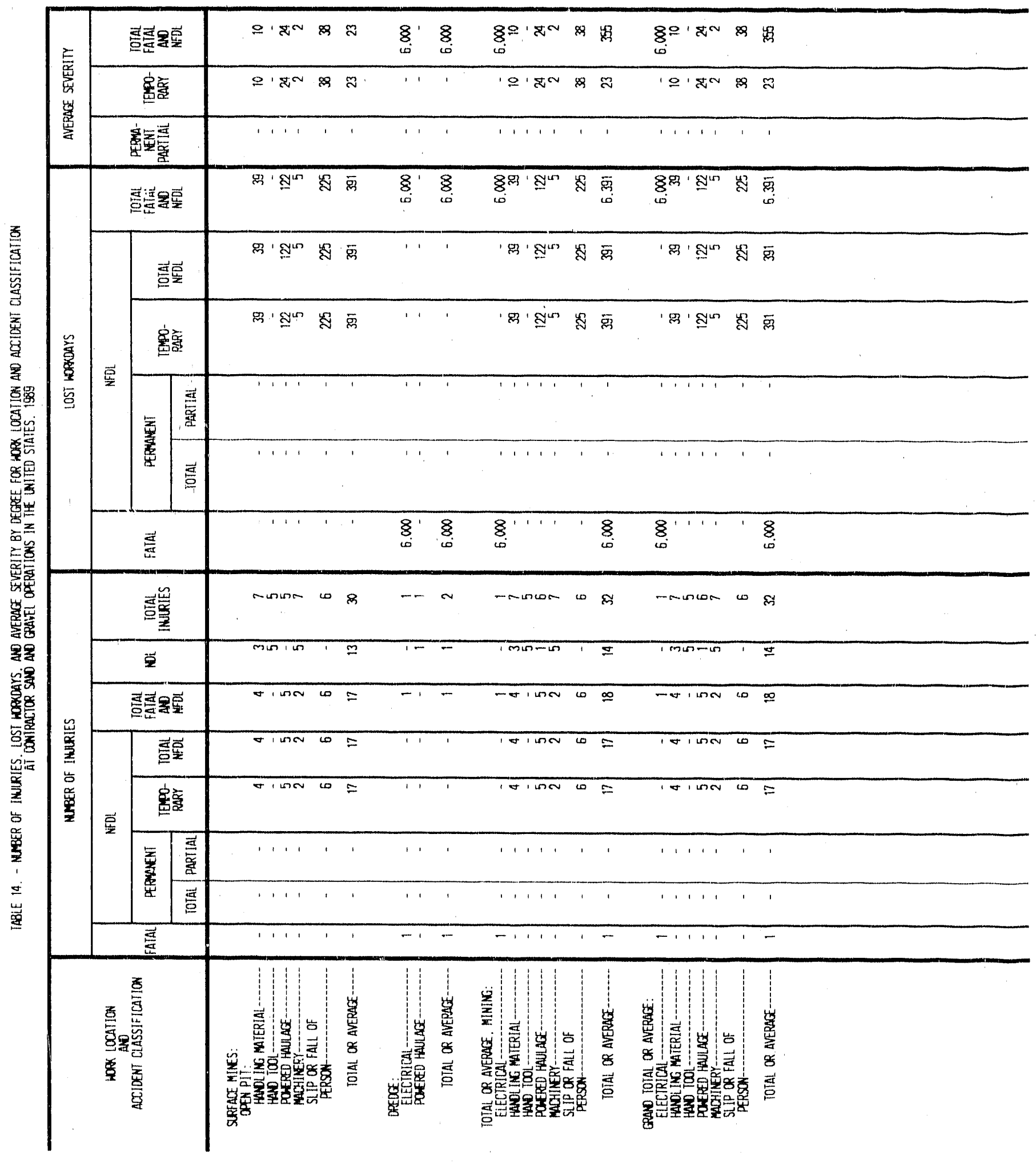




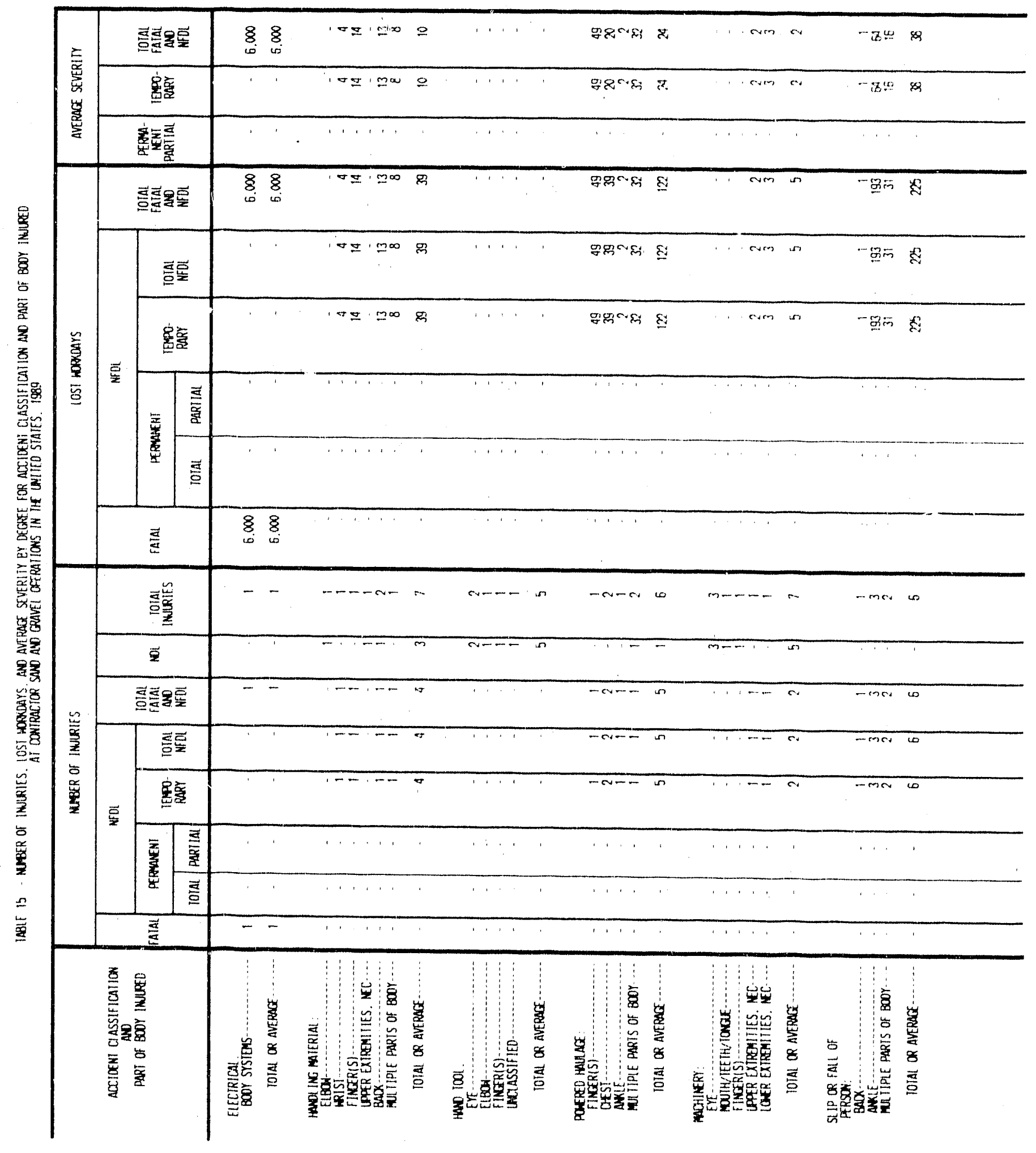




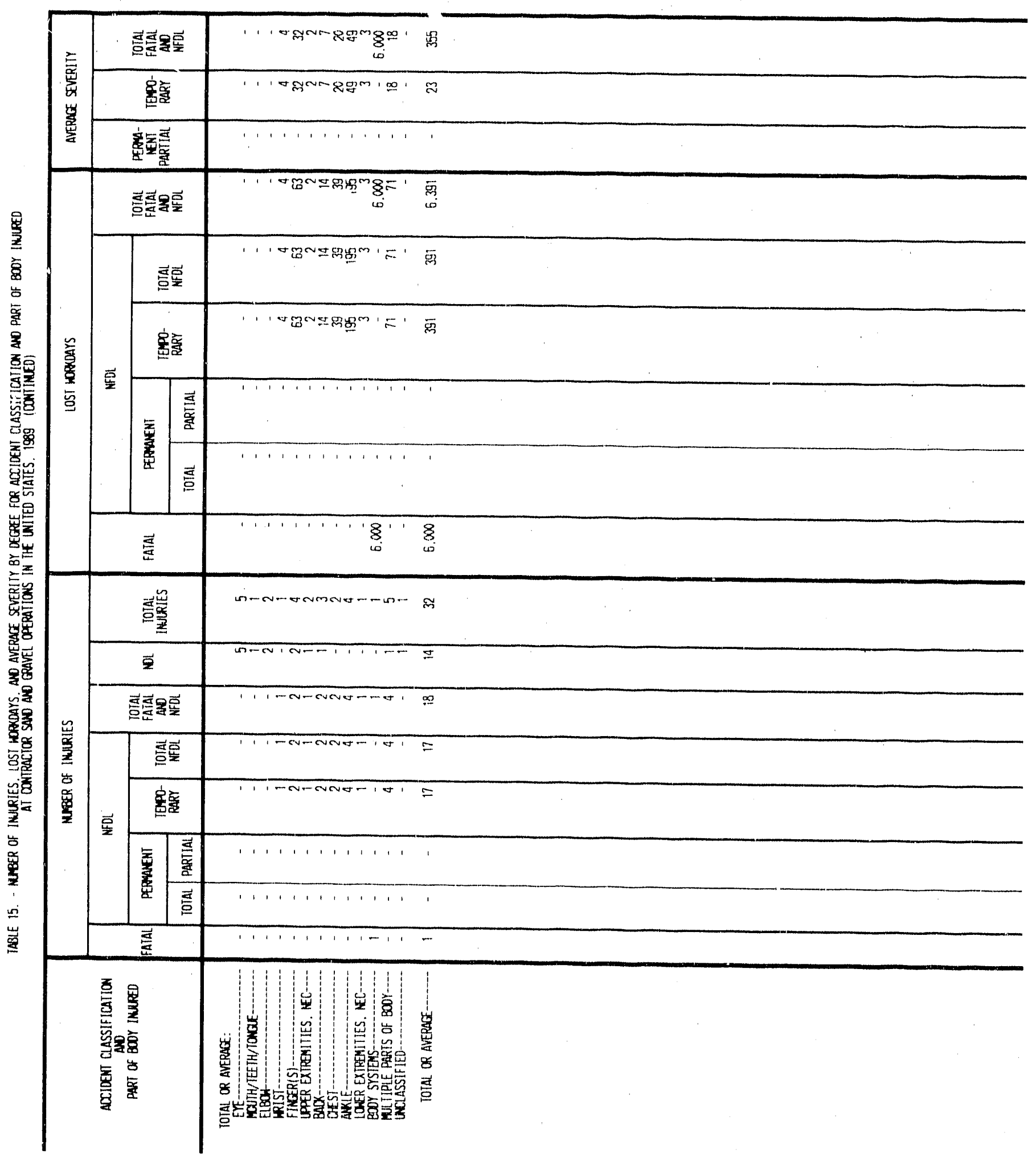




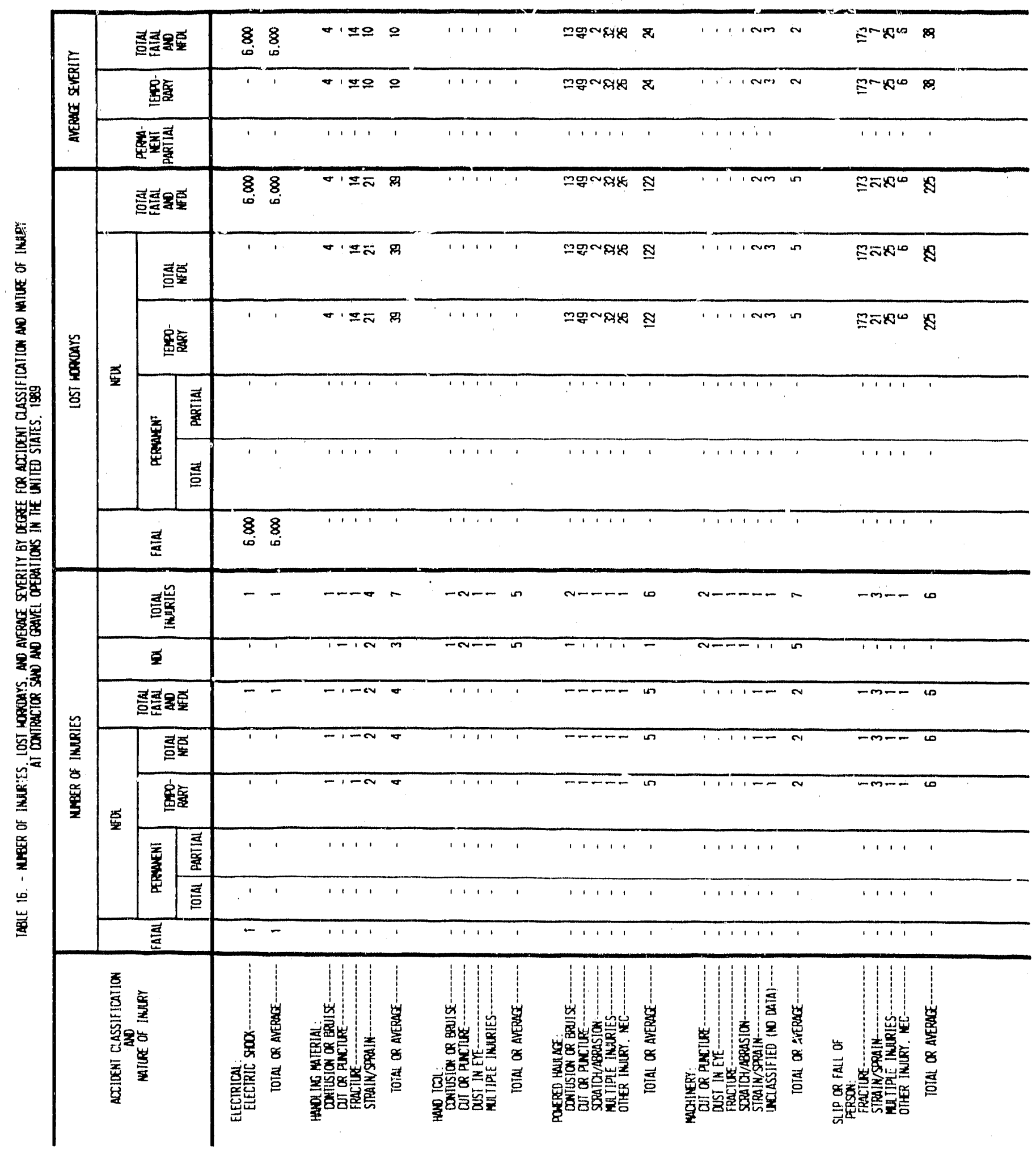




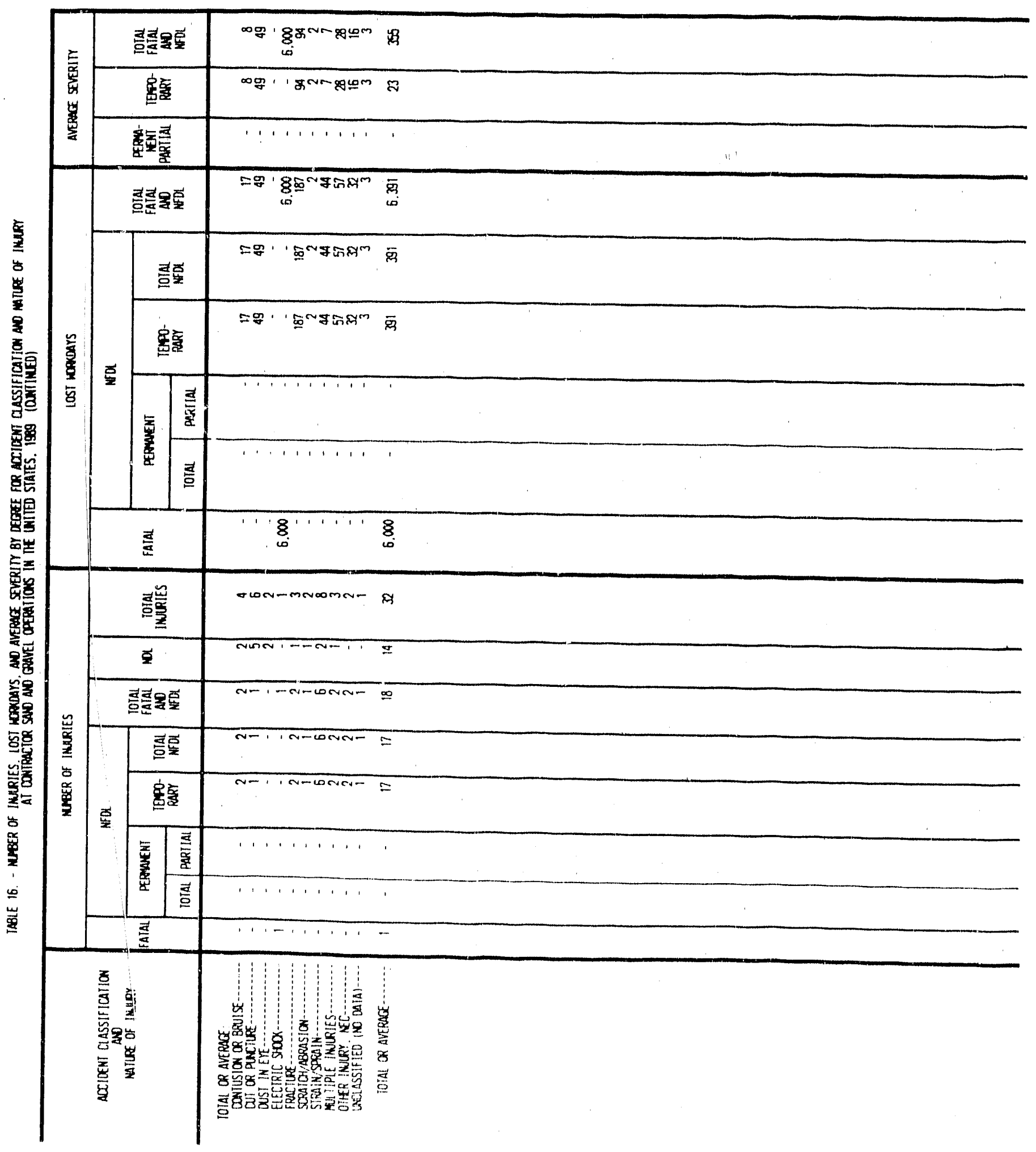




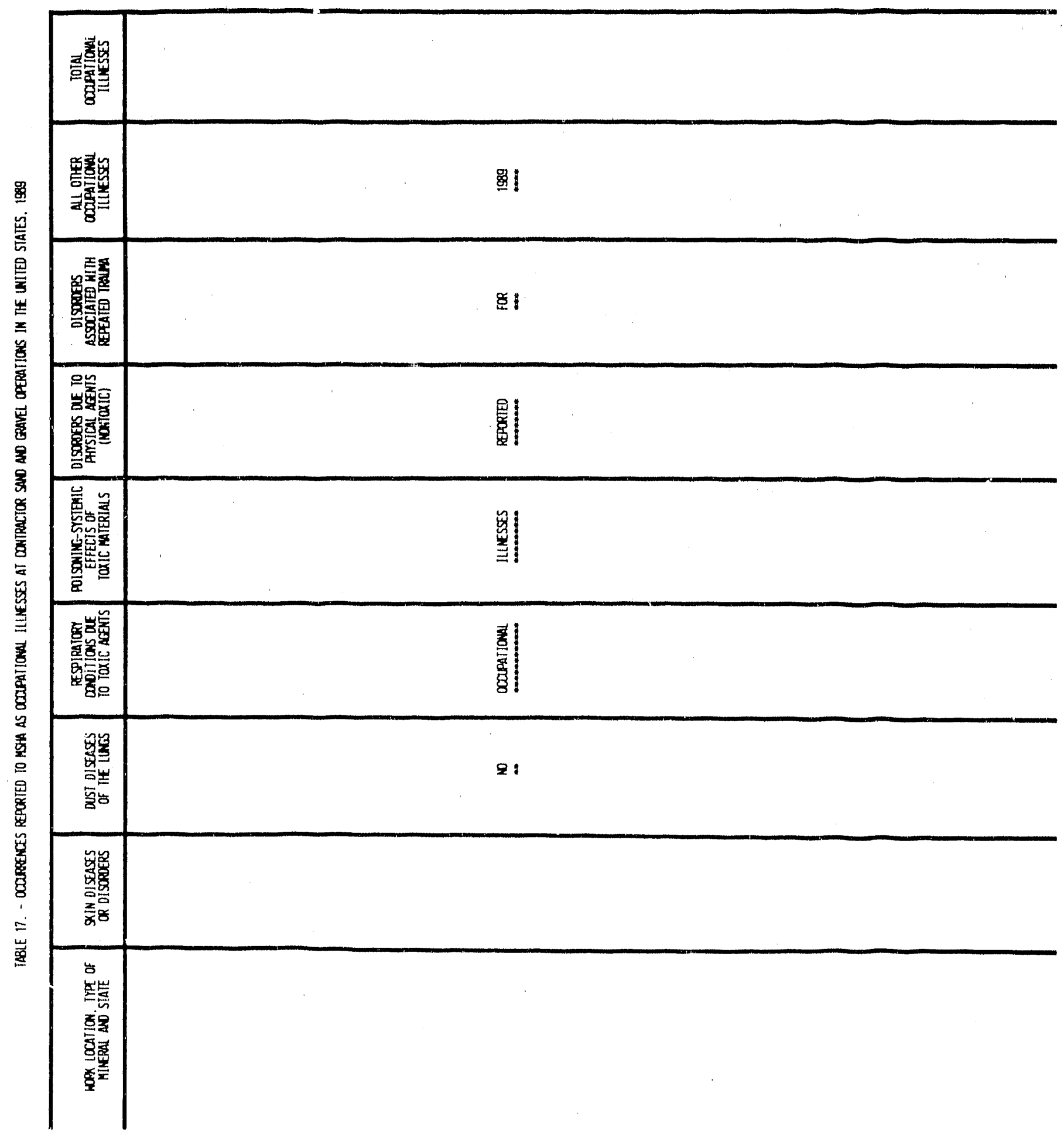




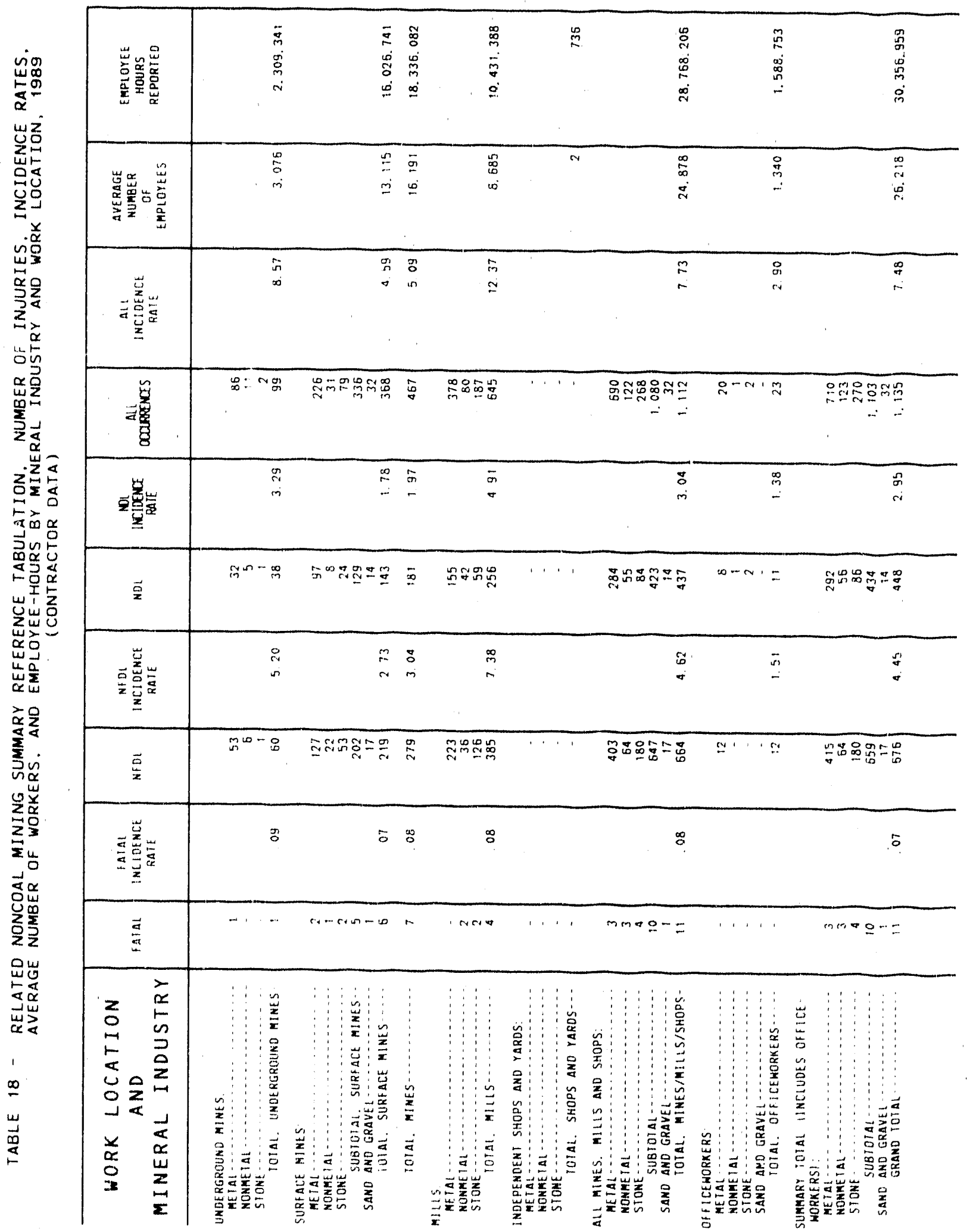




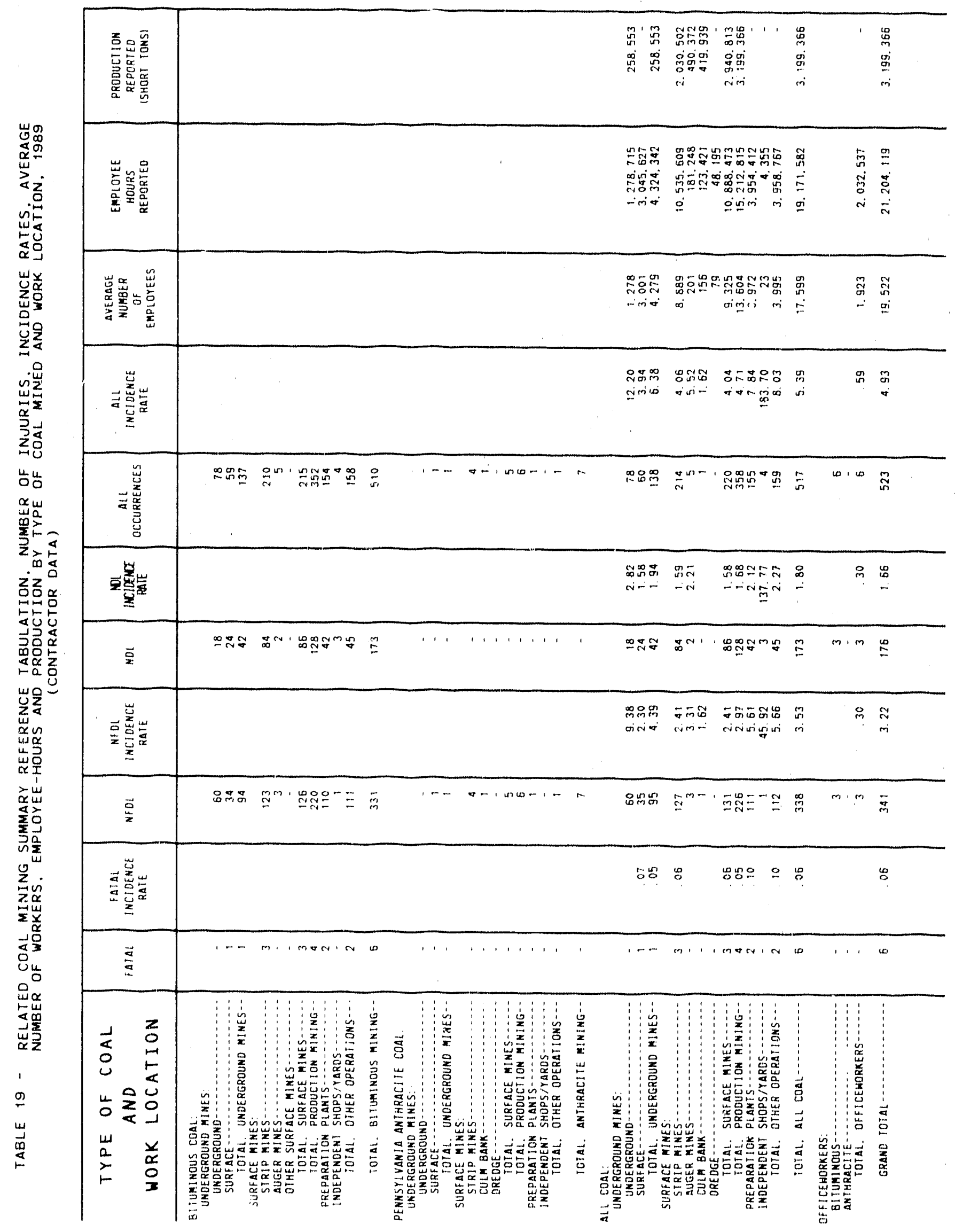

t4 US Government Prinfing Coffice $1990 \quad 773.004 / 23002$ 

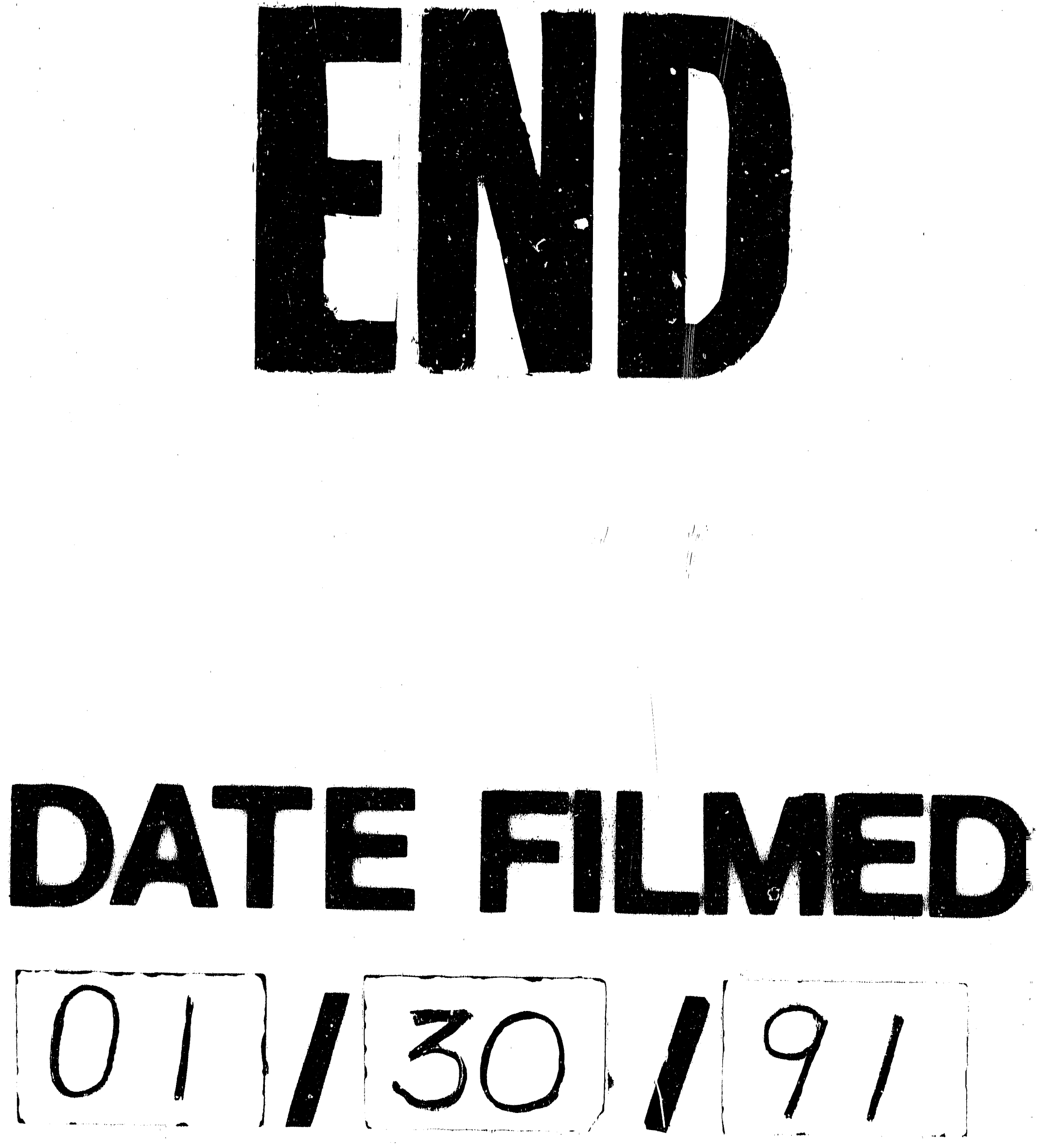
